NISTIR 7927

\title{
An Interlaboratory Investigation of the Effect of Material Granularity and Sample Processing on Measurement Variability: Nutritional Elements in Cereals
}

Katrice A. Lippa

Laura J. Wood

Stefan D. Leigh

N. Alan Heckert 
NISTIR 7927

\title{
An Interlaboratory Investigation of the Effect of Material Granularity and Sample Processing on Measurement Variability: Nutritional Elements in Cereals
}

\author{
Katrice A. Lippa \\ Laura J. Wood \\ Chemical Sciences Division \\ Material Measurement Laboratory \\ Stefan D. Leigh \\ N. Alan Heckert \\ Statistical Engineering Division \\ Information Technology Laboratory \\ http://dx.doi.org/10.6028/NIST.IR.7927
}

May 2013

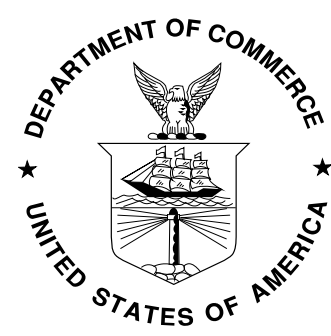

U.S. Department of Commerce Rebecca M. Blank, Acting Secretary

National Institute of Standards and Technology Patrick D. Gallagher, Under Secretary for Standards and Technology and Director 


\begin{abstract}
This interlaboratory study was specifically designed to determine the effects of material granularity and sample processing techniques on the measurement variability (i.e. precision) of nutritional elements in various cereal materials. Participants were asked to determine in triplicate the levels of iron, calcium and zinc in six breakfast cereals (three wheat-based, two rice-based and one wheat/rice mixture). Processing of the three ground materials (ground and homogenized wheat, ground wheat and ground rice) was anticipated to be relatively straightforward, whereas the two flake materials (wheat flake and rice flake) were expected to require appropriate processing prior to subsampling to limit measurement variability. The final material (wheat/rice mixture with a hand-crushed granularity) was anticipated to be a 'challenge' material due to its inherent heterogeneity both in content and in granularity. In general, approximately $25 \%$ of the laboratories processed and analyzed the suite of six cereal materials with adequate to exemplary measurement precision. Another $60 \%$ of the laboratories experienced measurement issues related only to a specific type of cereal matrix (e.g., wheat vs. rice or the 'challenge' wheat/rice sample), or for only a select element (e.g., Fe vs. Ca or Zn). A small number ( $\approx 15 \%$ ) of laboratories experienced significant sample processing and/or measurement problems. It should be noted that this study is a precursor to possible future exercises that will assess method precision using commercially available nutritional products that will contain more varied ingredients and thus exhibit significant heterogeneity.
\end{abstract}

\title{
INTRODUCTION
}

The Nutrition Labeling and Education Act of 1990 requires nutrition labels to report the Ca and Fe content of processed foods, including ready-to-eat cereal, that will be sold within the U.S. Nutrients such as Zn with an established reference daily intake value may also be listed. The nutritional elements study is designed to elucidate the effect of sample size/sample preparation as well as the individual testing laboratory comparability by analyzing a set of samples that range in granularity. This interlaboratory study was specifically designed to determine the effects of both the material granularity and the sample processing techniques on measurement variability as well as to provide individual laboratories information on their performance relative to the other participants (comparability information).

Six wheat-based and rice-based fortified breakfast cereals were selected as study materials (Figure 1) and were specifically prepared to represent a series of various granularities and fortification levels, as well as a range of fiber and protein content. Table 1 provides the nominal levels of the proximates (fats, protein, carbohydrates, etc.) as well as the levels of Fe, Ca and $\mathrm{Zn}$. As shown in Table 1, the rice-based cereal was fortified with Fe only but had naturally occurring levels of $\mathrm{Ca}$ and $\mathrm{Zn}$. Each laboratory was provided with the six cereal samples and asked to report values for triplicate measurements of Fe, $\mathrm{Ca}$ and $\mathrm{Zn}$ in each of the six samples. Laboratories were also asked to provide details regarding their sample processing techniques, amount of study material that was processed and the amount of subsample that was used in the analysis. 
As this study was designed to compare material granularities and sample processing techniques, a complete statistical assessment of between- and within-laboratory variabilities was conducted to determine the consistency of the data across the multiple sample processing approaches. The relative variabilities of triplicate measurements for $\mathrm{Fe}$, Ca and $\mathrm{Zn}$ for each of the six materials were assessed and compared with the sample processing information provided by each laboratory. Comparison with the sample processing information is important because the various matrices of this suite of cereal samples may respond differently to differing sample preparation techniques (e.g., mechanical grinding, hand-crushing), influencing an individual laboratory's ability to provide reproducible results for each material.

\section{SOURCE OF MATERIAL}

Cereal A (SRM 3233 Fortified Breakfast Cereal) was the only study material used that has NIST assigned values for $\mathrm{Fe}$, $\mathrm{Ca}$ and $\mathrm{Zn}$ and therefore the only material for which the measurement results can be assessed for trueness (or relative proximity to the NIST certified values).

Cereals B and C were prepared from the same source of commercially available fortified wheat flake cereal originating from two production lots that were well mixed prior to packaging (21 kg in total). Cereals D and E were prepared from the same source of commercially available partially fortified rice flake cereal originating from a single production lot (13 kg in total) (Figure 1). For both the wheat and rice cereals, half of the production lot was packaged directly as the flake material and the remainder was ground using a Teflon disc mill. Cereal $\mathrm{F}$ was prepared from a hand-crushed 50:50 mixture (by weight) of the wheat flake and rice flake cereals. While this material was well mixed, a visual examination of the individual packages of Cereal F suggested inhomogeneity with respect to both flake size and texture.

\section{SAMPLE DISTRIBUTION}

Duplicate samples from each of the six separate containers of cereals (Cereals A-F) containing approximately $30 \mathrm{~g}$ were provided to each participant and were distributed by the NIST laboratory in Charleston, SC to each of 20 laboratories (including NIST Gaithersburg, MD) during the week of April 12, 2010. These six cereals included three ground breakfast cereals, two whole flake breakfast cereals, and one partially crushed breakfast cereal. The ground and partially crushed materials were provided in sealed amber bottles, while the whole flake materials were supplied in sealed plastic bags.

Participants were advised to store samples at room temperature $\left(\approx 23^{\circ} \mathrm{C}\right)$ away from direct light prior to analysis. Participants were also advised about the general nutritional element levels in the samples, which were described as containing significantly varying Ca levels ranging from $300 \mathrm{mg} / \mathrm{kg}$ to $40000 \mathrm{mg} / \mathrm{kg}$, Fe levels from $250 \mathrm{mg} / \mathrm{kg}$ to $800 \mathrm{mg} / \mathrm{kg}$, and Zn levels from 30 $\mathrm{mg} / \mathrm{kg}$ to $700 \mathrm{mg} / \mathrm{kg}$. 


\section{EVALUATION OF EXERCISE RESULTS}

\section{Establishment of the Exercise Values}

Laboratory data submission: Each laboratory was requested to provide triplicate values for Ca, Fe and Zn for each of the six materials (Cereals A-F). Participants were advised to report data for all six samples and it was specified that it was preferred that the data be reported for all three analytes (a total of 18 values reported). However, if it was not feasible for a particular laboratory to provide the complete data, then it was requested they provide data for only a single element across all six materials rather than for a random selection of materials (e.g., it was sufficient to report Fe data for the six samples, rather than report all elements for only three samples). Laboratories were also asked to provide all sample preparation data to determine the effects of sample size and sample pretreatment. An example of the reporting sheet for the sample preparation data is provided in Appendix A.

A summary of the sample processing techniques used by each laboratory for processing of the flake and hand-crushed cereals C, E and F is provided in Table 2. Reported sample processing techniques included mortar and pestle, metal mechanical grinder, non-metal mechanical grinder, hammer and hand crushing. Approximately half (45\%) of the laboratories used a mortar and pestle, while $40 \%$ of the participating laboratories used a metal mechanical grinder. There were only three laboratories (15 \% of participating laboratories) that used other techniques: hand crushing, a hammer and a non-metal mechanical grinder. The majority (90\%) of laboratories processed the study material before subsampling for triplicate analyses. The amount of study material that was processed ranged from $1 \mathrm{~g}$ to $60 \mathrm{~g}$ ( 2 packages processed together). For only the three textured samples (Cereals $\mathrm{C}, \mathrm{E}$ and $\mathrm{F}$ ), the range of subsample size used for triplicate analysis was $0.2 \mathrm{~g}$ to $10 \mathrm{~g}$, with a median value of $0.5 \mathrm{~g}$.

Determination of laboratory analyte means and standard deviation: For each laboratory, the analyte mean and standard deviation of the three sample results were calculated for $\mathrm{Ca}$, Fe and $\mathrm{Zn}$ in each of the six materials (Cereal A-F). This data is provided in Table 3. In a few instances, laboratories provide identical values for the triplicate measurements therefore a standard deviation was labeled as "not applicable" or n/a. For laboratories that did not provide data for a particular analyte, the data was labeled as "not determined” or n/d.

Data distribution assessment: Normal probability plots for each material/analyte combination were generated to test if each dataset is normally distributed (Appendix B). Normal probability plot correlation coefficients (PPCC) and the $5 \%$ critical values (CV) were determined for each material/analyte combination. Normality is rejected at the $5 \%$ level if the PPCC value is less than the critical value. These plots were used as a data screening tool only and were used primarily to identify outlying laboratories.

Comparison to material NIST values: The NIST value for Cereal A (SRM 3233 Fortified Breakfast Cereal) was provided based on a combination of results from inductively coupled plasma optical emission spectrometry (ICP-OES) measurements made at NIST and from determinations made by external collaborating measurement laboratories. The mean value of the NIST measurements was combined with the median of the means of the collaborating 
laboratories to provide the NIST value. SRM 3233 was not certified at the time of this study therefore the study values may differ from the certified values. A comparison of participant's results for Ca, Fe and Zn in Cereal A (SRM 3233 Fortified Breakfast Cereal) to the NIST value at the time of this study is provided in Figure 2. Twenty laboratories participated in the nutritional elements in breakfast cereals sampling exercise including NIST. Participants used both ICP-OES and inductively coupled plasma mass spectrometry (ICP-MS) as the analytical techniques for the determination of these three elements. Fourteen of the non-NIST laboratories used ICP-OES and five used ICP-MS.

Determination of variability between- and within-laboratories: The triplicate data for $\mathrm{Fe}, \mathrm{Ca}$ and Zn for all six materials were analyzed with respect to between-laboratory (using an $h$-consistency statistic) and within-laboratory (using a $k$-consistency statistic) components of variance using the established ASTM E691 protocol for the determination of precision of a test method. ${ }^{1}$ Briefly, the between-laboratory consistency statistic $(h)$ is:

$$
h=d / s_{x}
$$

where $d$ is the individual laboratory deviation (deviation of the average of the individual laboratory (i.e., cell) values from the average of all the laboratories averages) and $s_{x}$ is the standard deviation of the laboratory (cell) averages. The within-laboratory consistency statistic $(k)$ is:

$$
k=s / s_{r}
$$

where $s$ is the standard deviation for an individual laboratory and $s_{r}$ is the repeatability standard deviation of the material, simply determined as:

$$
s_{r}=\sqrt{\sum_{1}^{p} s^{2} / p}
$$

with $p=$ the number of laboratories evaluated. The values of the $h$-consistency and $k$ consistency statistics determined for each data set are then compared to critical values of $h$ and $k$ at the $5 \%$ significance level.

The complete set of $h$-consistency statistic and $k$-consistency statistic plots for Fe, Ca and Zn in both sets of materials (Cereals A, B, C and Cereals E, D, F) together with a tabular data output are provided in Appendix C.

\section{REPORTED RESULTS}

An overview of the variability of the participating laboratories' performance for all 18 nutrient/cereal pairs is summarized in Table 4. The variability is tabulated as the median of the relative standard deviations (RSDs) of the results observed in each laboratory, where the relative standard deviation is defined as the standard deviation divided by the absolute value of the mean. The median RSD summary results illustrate that half (50 \%) of laboratories analyzed the 
triplicate samples with reasonable precision, having RSDs of $5 \%$ or less. For some laboratories, however, the results are less precise, with RSDs of $10 \%$ or greater, and in some cases, RSDs as high as $45 \%$.

Figure 2 compares the participant's results for $\mathrm{Ca}, \mathrm{Fe}$, and Zn to the independently measured NIST reference values for Cereal A. As shown in Figure 2, 15 out of 19 participating laboratories (79 \%) have sample mean values that fall within within the $95 \%$ confidence bounds $( \pm 2 u$ ) for the NIST value for Fe. For Ca, 9 out of 19 participating laboratories (47 \%) fall within the $95 \%$ confidence bounds and for Zn, 7 out of 20 participating laboratories (35\%) fall within the $95 \%$ confidence bounds. The uncertainty associated with Fe for the NIST value was greater than for Ca or Zn. ${ }^{6-8}$ The larger uncertainty associated with the NIST value for Fe, with a relative expanded uncertainty of $12 \%$, afforded the participants a greater opportunity of falling within the target range. The relative expanded uncertainties associated with the NIST values for Ca and $\mathrm{Zn}$ were $2 \%$ and $4 \%$ respectively.

Normal probability plots for each material/analyte combination (Appendix B) were assessed to evaluate if each dataset were normally distributed. Specifically for the Ca data, the cereal A measured mass fractions are approximately normally distributed. For cereals B and C (wheat ground and wheat flake), the PPCC values are just below the $5 \%$ critical value, so the departure from normality is mild. For cereal B, a number of laboratories have slightly higher than expected mass fractions while for cereal $C$ there are 4 laboratories with somewhat lower than expected mass fractions. Cereals D, E, and F (rice ground, rice flake, rice/wheat crushed) have PPCC values that are well below the $5 \%$ critical value. For cereals D and E (rice ground and rice flake), there are several laboratories with slightly higher than expected mass fractions and three laboratories with much higher than expected mass fraction values. It is notable that these two materials have considerably lower mass fraction of Ca (nearly 100-fold) than the other materials. The source of calcium in these rice cereals originates from its naturally-occurring form within the cereal matrix, calcium caseinate, which is added to the cereal for protein fortification. Notably, this amount of calcium added does not provide a significant amount of Ca with respect to the recommended daily allowances per serving size of $30 \mathrm{~g}$. This is in contrast to the wheat cereals in which Ca fortification is achieved by the direct addition of calcium carbonate.

For the Fe data, only cereal D (rice ground) appears to be approximately normal. Cereals A and $\mathrm{B}$ (superground wheat and ground wheat) have a number of laboratories with much lower than expected mass fractions (more severe for cereal A). Cereals E and F (rice flake and rice/wheat crushed) have lower than expected mass fractions in the lower half of the data.

Lastly for Zn, cereals A, B, and F are approximately normal. The PPCC value for cereal C is just below the $5 \%$ critical value, so the departure from normality is mild (there is one lab with a much lower than expected mass fraction). Cereals D and E (rice ground and rice flake) have several laboratories with much lower than expected mass fractions and several laboratories with much higher than expected mass fractions. Similar to the Ca data, the mass fraction of $\mathrm{Zn}$ in these two materials is considerably lower (nearly 20-fold) than the other cereals and is present in only its naturally-occurring form within the cereal matrix. 
Examples of $h$ and $k$ plots for the determination of $\mathrm{Zn}$ in the ground and flake wheat cereals (A, $\mathrm{B}$ and $\mathrm{C}$ ) are shown in Figure 3. In the top figure, laboratories considered to be significantly different relative to the other laboratories ( $h$-consistency statistic) will approach or fall above or below the critical value of $|2.56|$ (5 \% significance level). For laboratories that have not adequately processed all samples, a greater between-laboratory variance would be expected for Cereal C (wheat flake) in comparison to Cereals A and B (wheat ground with homogenization and wheat ground, respectively) because the variability of values determined for Cereal $\mathrm{C}$ are more dependent on the completeness of sample processing. The data given in the top figure indicate, however, that most laboratories have similar $h$-consistency statistics for the three wheat materials, implying that all samples were adequately processed and that the method of processing the material is not contributing significantly to the overall measurement variability for Zn.

In the bottom figure, the $k$-consistency statistics of laboratories that approach or fall above the critical value of 2.21 (5 \% significance level) denote significantly greater within-laboratory variability for these measurements. However, while small $\mathrm{k}$ values are indicative of small variability, very small k values (near zero) may also indicate measurement problems (i.e. measurement insensitivity). ${ }^{2,3}$ For laboratories that have not adequately processed all samples, a greater within-laboratory variance ( $k$-consistency statistic) would be expected for Cereal C (wheat flake) in comparison to Cereals A and B (wheat ground and homogenized and wheat ground, respectively). The data given in the bottom figure indicate, however, that most laboratories have similar $k$-consistency statistics for the three wheat materials, reinforcing the hconsistency conclusion (top figure) that most samples were adequately processed and that the method of processing the material is not contributing significantly to the overall measurement variability for $\mathrm{Zn}$.

Six sets of $h$ - and $k$-consistency plots similar to Figures 3a and 3b were generated (one for each element and material combination) and are provided in Appendix C. A summary of those data that were above the critical line (5\% significance level) are provided in Table 5 . This summary table (Table 5) illustrates which laboratories are outliers with respect to the h and $\mathrm{k}$ indicators for the three elements (Fe, $\mathrm{Ca}$ and $\mathrm{Zn}$ ) across the six materials investigated. The several $h$ indicators for $\mathrm{Zn}$ in Cereal F may be representative of sample heterogeneity. The few $h$ indicators randomly scattered across materials and elements could indicate anything from improper sample handling or preparation to an error in data processing. The clusters of $k$ indicators around labs and materials are sometimes inconsistent and were used only to flag outlying data.

Figure 4 represents exemplar consensus plots for $\mathrm{Zn}$ in the ground and flake wheat cereals (Cereal B and Cereal C, respectively) using DerSimonian-Laird (DSL) weighted consensus means and multiples of Horn-Horn-Duncan (HHD) standard uncertainty lines. The DSL weighted mean is a method commonly used in the biostatistical literature to determine a consensus value from many different participating laboratories' measurements. The DSL weighted consensus mean accounts for both the within- and between-laboratory variance components and is used to determine interlaboratory consensus values. ${ }^{4}$ Horn-Horn-Duncan (HHD) is a related method used to estimate consensus uncertainty of the mean. ${ }^{5}$ The examples in Figure 4 show the relationship of each laboratory's three individual $\mathrm{Zn}$ values to the consensus mean. The solid line connecting the median value illustrates the replicate variability within a laboratory. 
A complete set of eighteen DSL consensus plots are provided for each element (Fe, Ca and Zn) in each of the six materials (Cereals A-F) in Appendix D. The results of these consensus plots were compiled by generating summary consensus plots that represent the performance of each individual laboratory with respect to the overall exercise results. The individual laboratorycustomized plots are provided in Appendix E with explanatory details intended to provide useful feedback regarding each laboratory's performance in this study.

\section{DISCUSSION}

A few general observations were found based on results obtained from the 20 participants in this study. While each participant's data is unique to his or her own laboratory, there are a variety of patterns and trends that are identifiable based on results obtained from the 20 participants. Technical guidelines can be recommended for good laboratory practices based on these observations.

It was observed that laboratories processing samples in their entirety produced a more homogeneous material that could be subsequently subsampled for further analysis. In general, the optimal procedure is to process (i.e., grind, crush) the entire sample received first (i.e., the entire bottle or package) then subsample for analysis after processing the sample. Also, when analyzing food and dietary supplements, complete digestions are required to ensure that all elements are in solution in order to obtain accurate results. Leachate-type digestions will not produce complete digestions for food or dietary supplements and results for these types of digestions were found to be below the NIST target value.

The use of calibration standards, obtained from a reliable source, must still be confirmed for quality before use. Determination of the approximate mass fraction of the working solutions will allow calibration points to be made that closely surround the working solutions, ensuring that calibration points are linear in the measurement range allowing for more accurate measurements. Along with unknown samples, an appropriate Certified Reference Material (CRM) or traceable in-house control should be prepared and carried through the entire experiment for quality assurance.

Some initial comparisons can be made with this data of the sampling processes that were used by each laboratory (Table 2). While it is not readily apparent that the sample processing method grossly affects analytical outcome, it does appear that both when the processing was employed (grinding before or after subsampling for analysis) and the amount of sample used for grinding can influence the between- and within-laboratory variability. Between-laboratory differences ( $h$ consistency statistic) observed in flake materials may be an indication of material inhomogeneity potentially caused by improper sample processing, sampling a very small amount or processing the sample after it has been subsampled for analysis. When extreme values are observed ( $h$ consistency statistics) for both ground and flake materials, this may be an indication of a potential calibration or dilution error. The majority of laboratories (70 \%) indicated that the entire bottle or package was used for grinding prior to subsampling. Partitioning the original sample into relatively small subsamples prior to grinding and homogenization may result in 
obtaining replicate subsamples that differ in granularity, nutrient and thus elemental content. When outliers are observed due to variability within a laboratory's measurements ( $k$-consistency statistic), methodological sources of error such as improper sample processing, sample analysis, improper calibration or incomplete digestion are possible. These factors can contribute to a significant increase in variability.

The greatest variability was observed for measurement of $\mathrm{Ca}$ in cereals $\mathrm{D}, \mathrm{E}$, and $\mathrm{F}$, which are rice-based materials that contained native levels of Ca. Likewise, Cereal F is comprised of $50 \%$ of the rice-based cereal (Cereal D/E) with the other $50 \%$ comprised of the wheat-based cereal (Cereal B/C). Cereals D and E also contained a higher protein content and this particular material and element combination may have caused more difficulty for some laboratories with respect to sampling and/or chemical analysis. In contrast to Ca, most laboratories sampled and analyzed Fe with essentially the same degree of precision independent of the sample matrix (similar across the six materials). Some laboratories had difficulty with Fe for all of the sample matrices, which may be attributable to the fact that Fe contamination is more prevalent than either $\mathrm{Ca}$ or $\mathrm{Zn}$ in a typical laboratory environment. Such contamination could have occurred during either the sample processing or chemical analysis steps and care should be taken to avoid Fe contamination. The variability of Zn measurement was similar to that of Ca measurement in the wheat based materials and appeared more manageable to the laboratories with respect to their overall precision with observed RSDs typically less than $6.5 \%$.

Increased variability may also be a product of material heterogeneity, as in Cereal F. Betweenlaboratory results for the overall $\mathrm{Fe}$, $\mathrm{Ca}$ and $\mathrm{Zn}$ levels in Cereal $\mathrm{F}$ may thus be more variable among the participating laboratories, with some laboratories reporting much higher (or lower) nutrient levels for Cereal $\mathrm{F}$ depending on whether the mixture contained more of Cereal $\mathrm{C}$ or Cereal E. As a case in point, this material heterogeneity may explain why some of the laboratory results (including NIST's) for Cereal F for all three nutrients (Fe, Ca and Zn) were considerably different from the consensus value. Nonetheless, Cereal $F$ is a practical example of a material that laboratories may be required to evaluate, in which values from replicate bottles or packages do not agree as a result of material inhomogeneity. An important consideration when faced with inhomogeneity is determination of an appropriate sample size. For instance, if the sample to be measured were granola bars, one bar would most likely not constitute a representative sample. An entire box of granola bars may not even constitute a representative sample. Laboratories must determine on a case-by-case basis the amount of material needed to have a representative sample for evaluation. In this cereal study, between-laboratory results tended to be more variable for Cereal F. Within-laboratory results for triplicate measurements should not be affected if appropriate sample processing and subsampling techniques are employed.

\section{Acknowledgments}

The time and effort of the analysts and management of the participating laboratories are gratefully acknowledged. We thank Rebecca S. Pugh, Amanda J. Moors, and Lauren B. Rust of the Hollings Marine Laboratory for preparation and shipping of the cereal samples to the participants. We also thank David L. Duewer for invaluable data visualization advice and 
Catherine A. Rimmer, Melissa M. Philips and Katherine E. Sharpless for their helpful suggestions and editorial advice.

\section{Disclaimer}

Certain commercial equipment, instruments, or materials are identified in this report to specify adequately the experimental procedure. Such identification does not imply recommendation or endorsement by the National Institute of Standards and Technology, nor does it imply that the materials or equipment identified are the best available for the purpose.

\section{References}

1. ASTM E691-99: Standard practice for conducting an interlaboratory study to determine the precision of a test method. 1999.

2. Mandel, J. Evaluation of control measurements, New York: Marcel Dekker, 1991.

3. Rukhin, A. L. Weighted means statistics in interlaboratory studies. Metrologia, 46 (2009), 323-331.

4. DerSimonian, R., Laird, N. Meta-analysis in clinical trials. Control. Clin. Trials, 7 (1986), 177-188.

5. Horn, R.A., Horn, S.A., Duncan, D.B. Estimating heteroscedastic variance in linear models. J. Am. Stat. Assoc., 70 (1975), 380-385.

6. Sharpless, K. E. NIST Material Measurement Laboratory, Analytical Chemistry Division Report of Analysis 839.00-09-118.

7. Sharpless, K. E. NIST Material Measurement Laboratory, Analytical Chemistry Division Report of Analysis 839.00-09-118a.

8. Francini, L, Wood, L., NIST Material Measurement Laboratory, Analytical Chemistry Division Report of Analysis 839-01-033. 
Figure 1. Overview of the preparation and relative matrix differences for the six cereals (A-F)
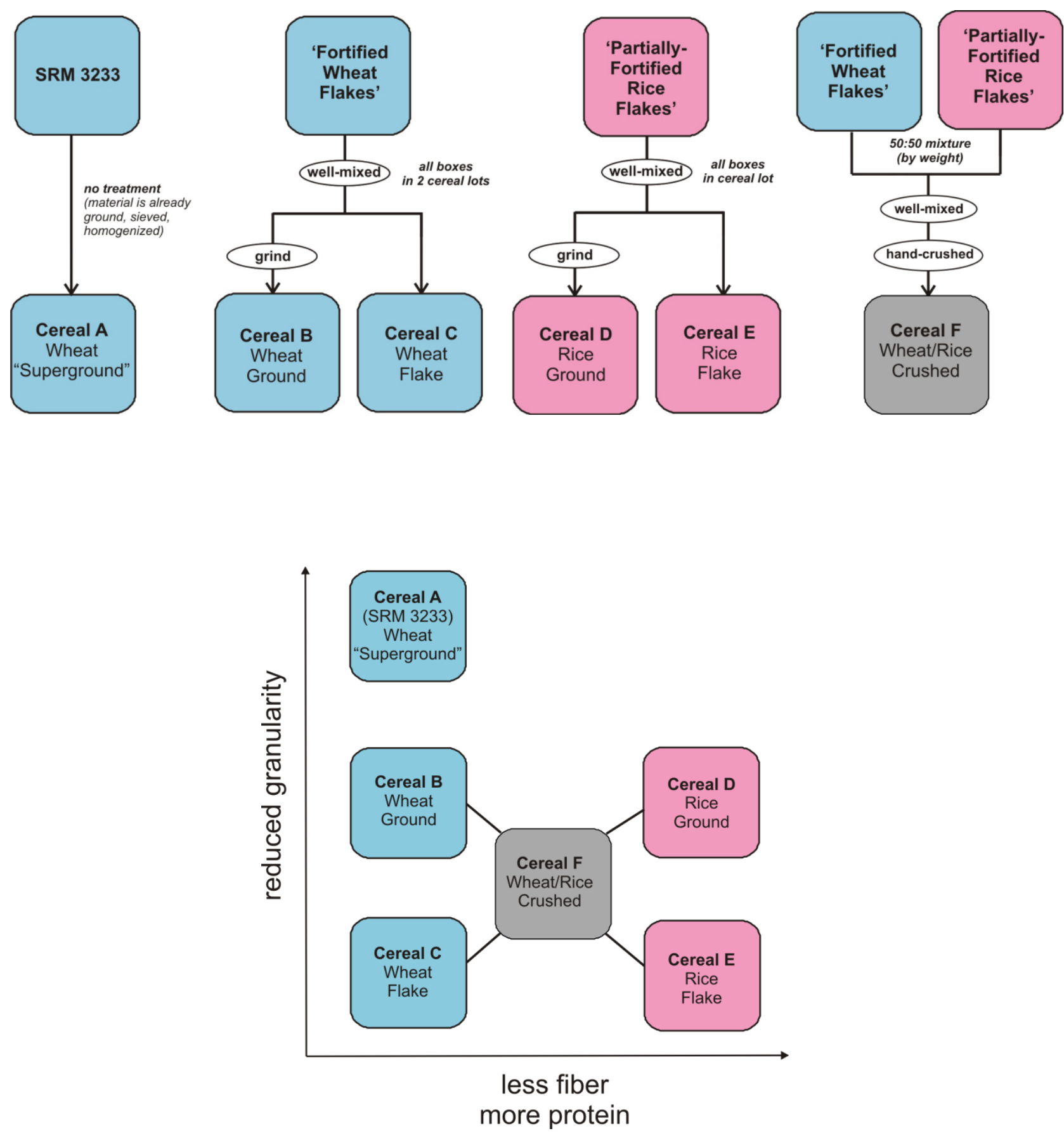
Figure 2. A comparison of participant results with independently measured NIST reference values for Ca, Fe and Zn (mg/kg) in "Cereal A" Superground Wheat (SRM 3233 Fortified Breakfast Cereal). The mean value for the triplicate data are represented as circles $(\bullet)$ with the corresponding vertical lines representing the range of the values (min to max) for each individual laboratory. The NIST reference values are represented by dark solid lines with corresponding \pm 1, 2, 3, 4, 5, 6 standard uncertainty $(u)$ lines. The approximate 95\% confidence interval $( \pm 2 u)$ about the NIST value is highlighted with a red-shaded box.

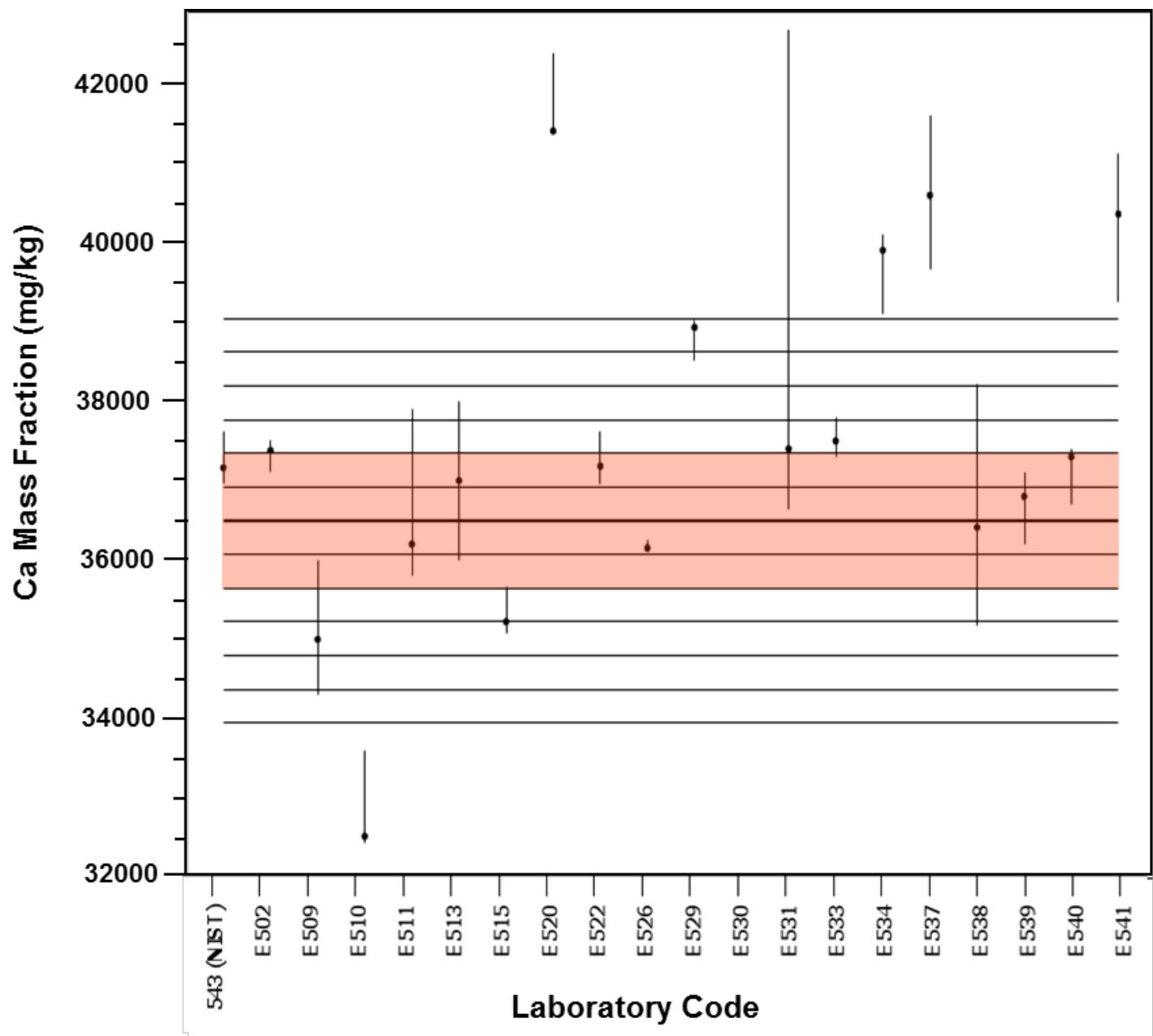

NIST Assigned Value $36500 \pm 850 \mathrm{mg} / \mathrm{kg}$ 
Figure 2 (cont'd).

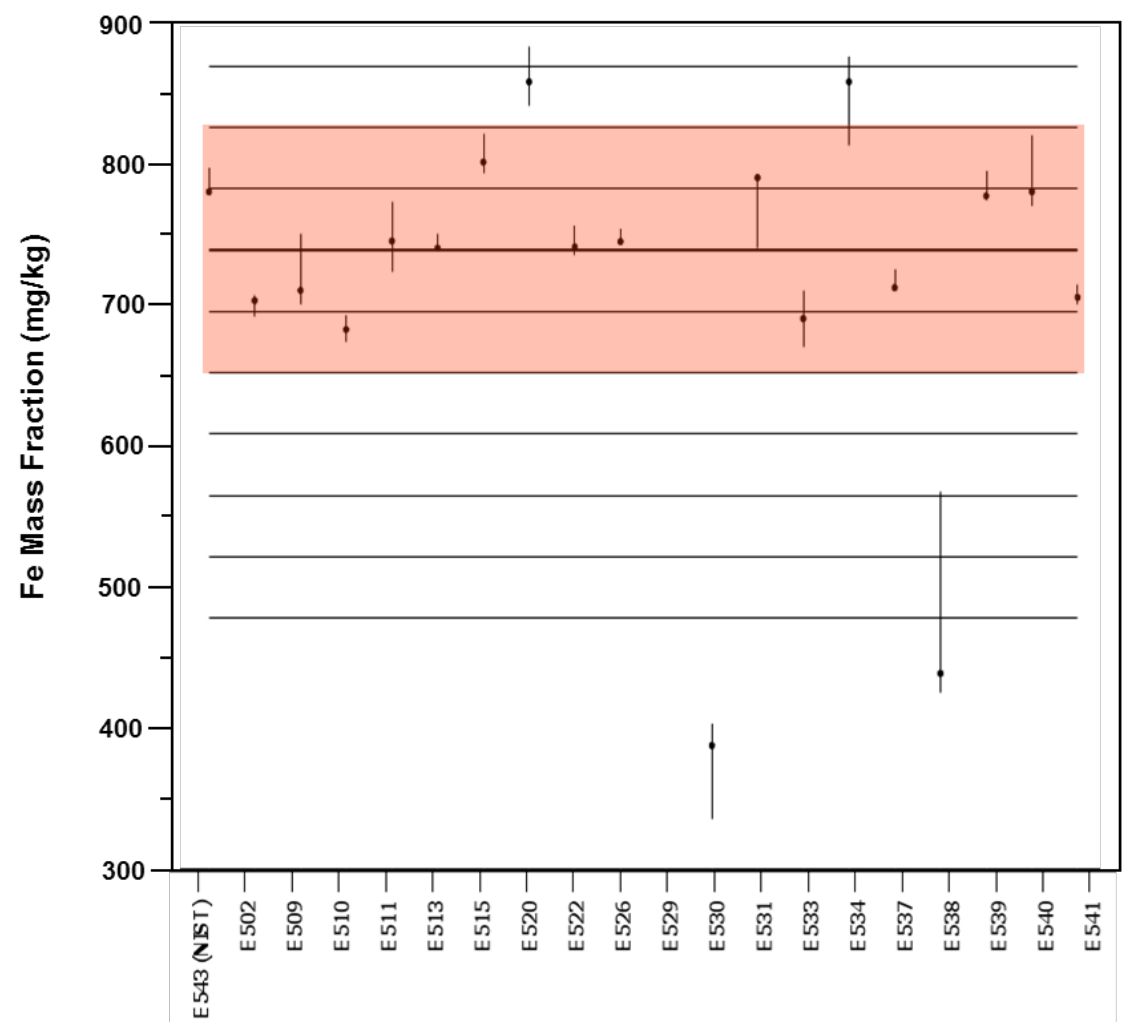

NIST Assigned Value

$738.9 \pm 87.1 \mathrm{mg} / \mathrm{kg}$

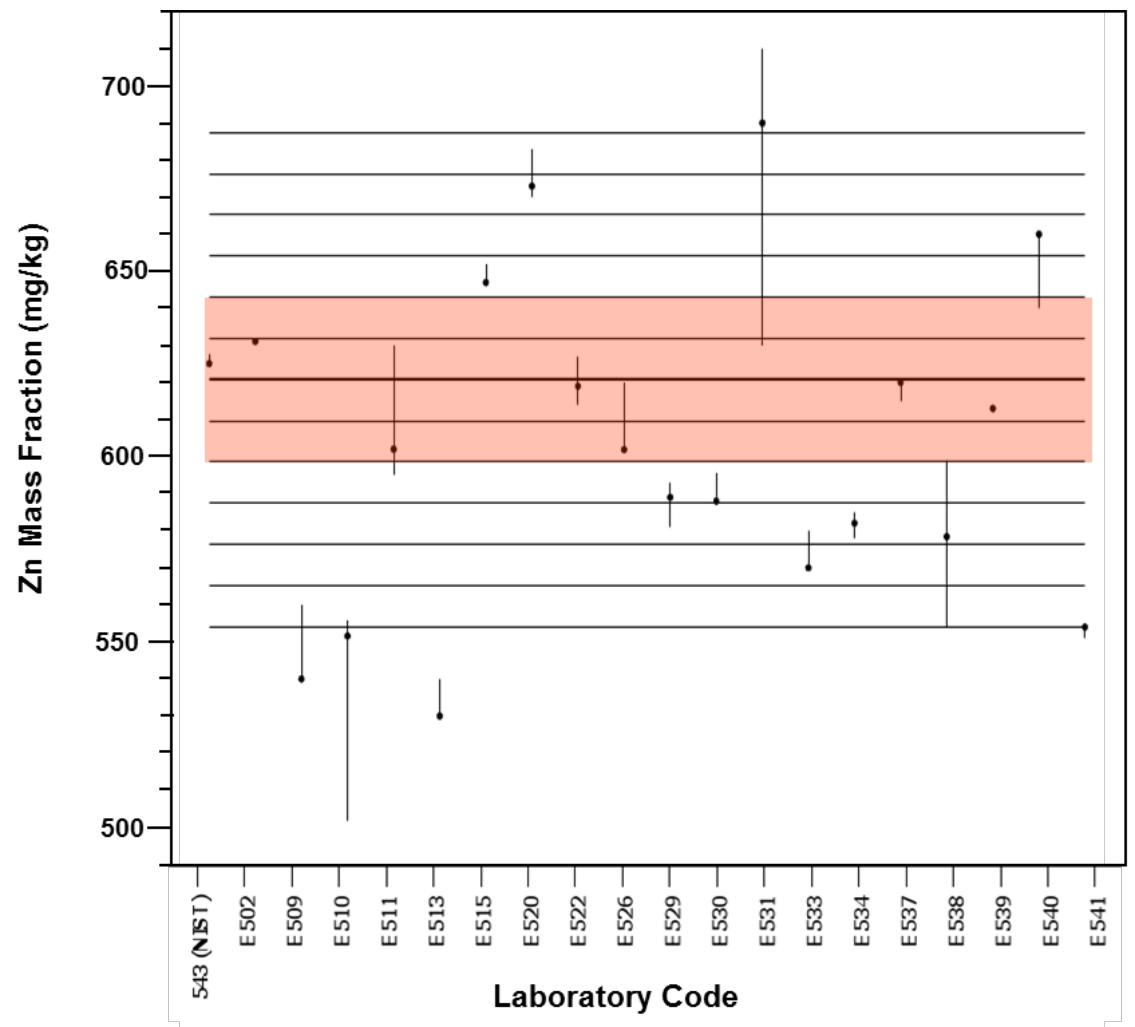

NIST Assigned Value $620.8 \pm 22.2 \mathrm{mg} / \mathrm{kg}$ 
Figure 3. Plots represent both the $h$-consistency statistics (between-laboratory) and $k$ consistency statistics (within-laboratory) for $\mathrm{Zn}$ in wheat cereals $\mathrm{A}, \mathrm{B}$ and $\mathrm{C}$ (wheat ground and homogenized, wheat ground and wheat flake, respectively). The $5 \%$ significance levels (critical values) are denoted by a dashed horizontal line for each plot.
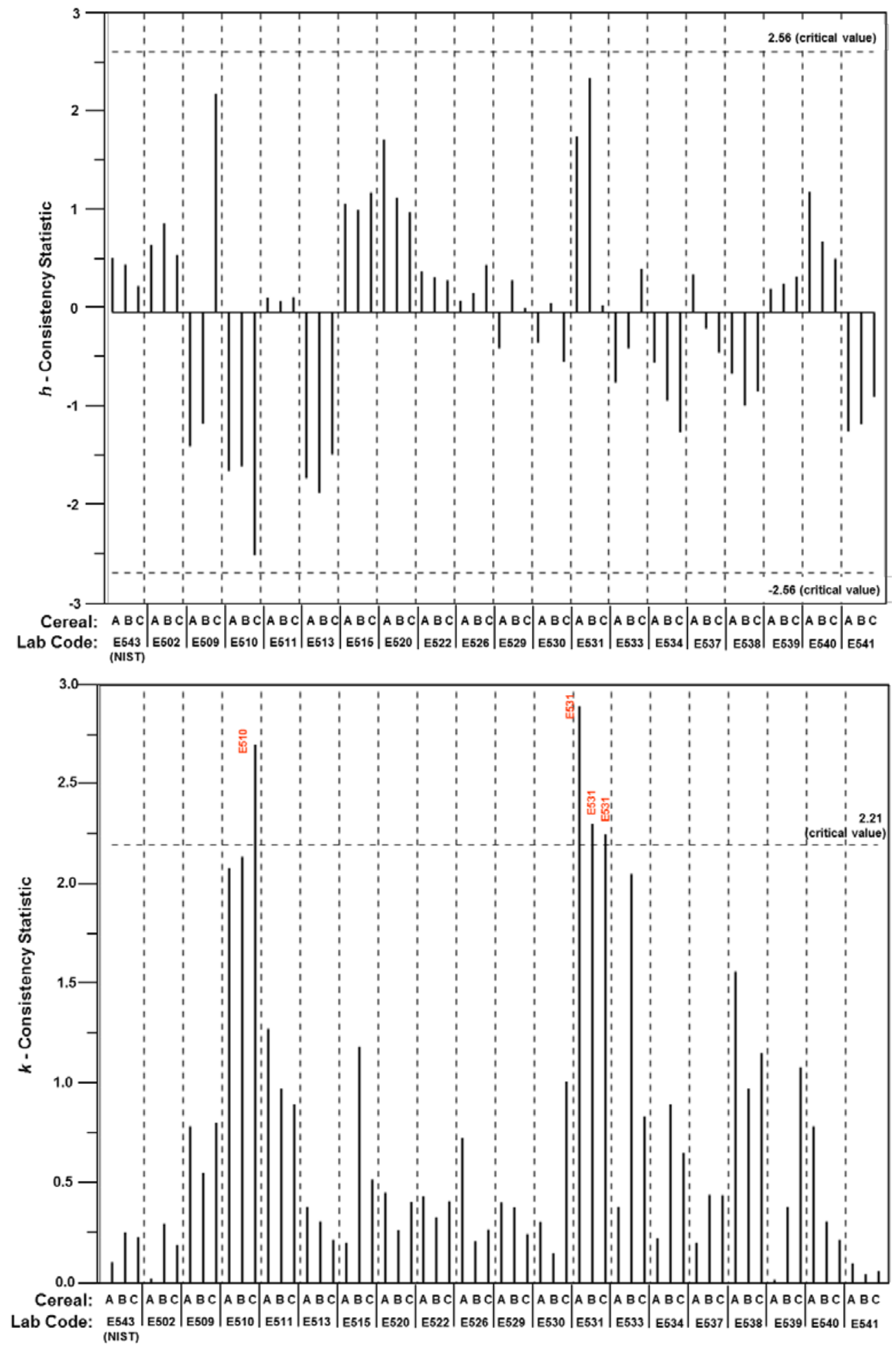
Figure 4. Consensus plots for $\mathrm{Zn}$ in Cereal B: wheat ground and Cereal C: wheat flake. The DerSimonian-Laird (DSL) consensus means are represented by the dark solid lines with $\pm 1,2,3$, 4, 5, 6 Horn-Horn-Duncan (HHD) standard uncertainty lines. The median value for the triplicate data are represented as circles $(\bullet)$ with the corresponding lines representing the range of the values (min-max) for each individual laboratory. Laboratory data that were flagged with a $k$ consistency statistic are labeled in red.

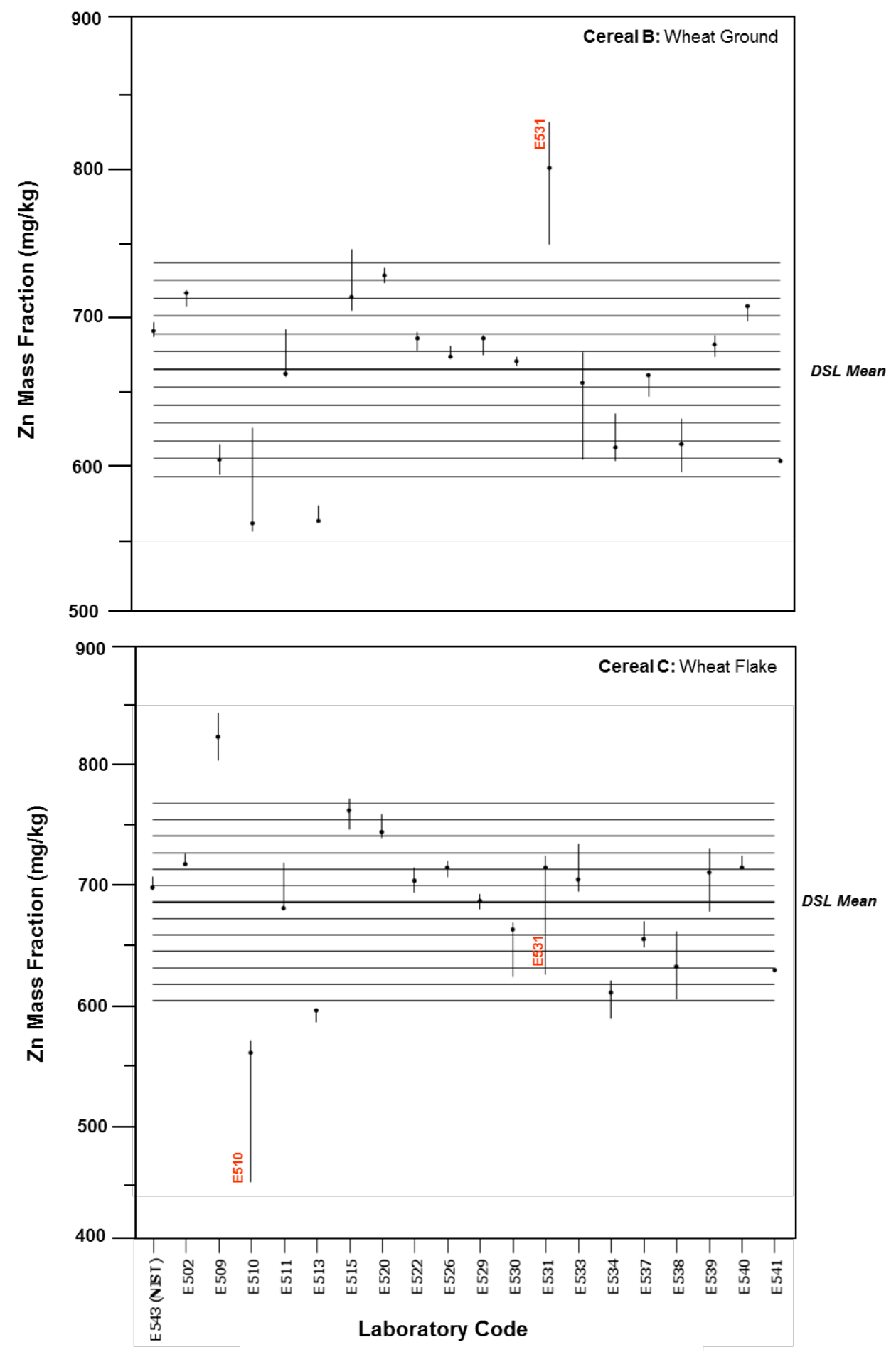


Table 1. Nominal nutrient levels for the six cereals (A-F).

\begin{tabular}{|c|c|c|c|c|c|c|}
\hline Nutrient & $\begin{array}{c}\text { Cereal A: } \\
\text { Wheat } \\
\text { Ground } \\
\text { (SRM 3233) }\end{array}$ & $\begin{array}{c}\text { Cereal B: } \\
\text { Wheat } \\
\text { Ground }\end{array}$ & $\begin{array}{c}\text { Cereal C: } \\
\text { Wheat } \\
\text { Flake }\end{array}$ & $\begin{array}{l}\text { Cereal D: } \\
\text { Rice } \\
\text { Ground }\end{array}$ & $\begin{array}{l}\text { Cereal E: } \\
\text { Rice } \\
\text { Flake }\end{array}$ & $\begin{array}{c}\text { Cereal F: } \\
\text { Wheat/Rice } \\
\text { Crushed }\end{array}$ \\
\hline Fiber (mg/g) & 100 & 100 & 100 & 33 & 33 & 67 \\
\hline Protein (mg/g) & 67 & 67 & 67 & 200 & 200 & 133 \\
\hline $\begin{array}{l}\text { Carbohydrates } \\
\text { (mg/g) }\end{array}$ & 770 & 770 & 770 & 770 & 770 & 770 \\
\hline Ca (mg/kg) & 36500 & 35000 & 35000 & 200 & 200 & 17500 \\
\hline $\mathrm{Fe}(\mathrm{mg} / \mathrm{kg})$ & 750 & 800 & 800 & 500 & 500 & 650 \\
\hline Zn (mg/kg) & 600 & 700 & 700 & 20 & 20 & 350 \\
\hline
\end{tabular}


Table 2. Sample preparation details for the three textured (non-ground) cereals (C, E, F).

\begin{tabular}{|c|c|c|c|c|c|c|}
\hline $\begin{array}{l}\text { Lab } \\
\text { Code }\end{array}$ & $\begin{array}{l}\text { Sample Processing } \\
\text { Method }^{\mathrm{a}}\end{array}$ & When ${ }^{\mathrm{b}}$ & $\begin{array}{l}\text { Subsample } \\
\text { Amount (g) } \\
\text { for Analyses }\end{array}$ & $\begin{array}{l}\text { Cereal C: } \\
\text { Wheat } \\
\text { Flake } \\
\text { Processed (g) }\end{array}$ & $\begin{array}{c}\text { Cereal E: } \\
\text { Rice } \\
\text { Flake } \\
\text { Processed (g) }\end{array}$ & $\begin{array}{c}\text { Cereal F: } \\
\text { Wheat/Rice } \\
\text { Crushed } \\
\text { Processed (g) }\end{array}$ \\
\hline E509 & metal mechanical grinder & before & 10 & 60 & 60 & 30 \\
\hline E510 & metal mechanical grinder & after & 0.2 & 60 & 30 & 30 \\
\hline E511 & metal mechanical grinder & before & 2.2 & all $^{\mathrm{c}}$ & all $^{\mathrm{C}}$ & all $^{\mathrm{C}}$ \\
\hline E520 & metal mechanical grinder & before & $\mathrm{n} / \mathrm{a}$ & 30 & 30 & 30 \\
\hline E522 & metal mechanical grinder & before & 0.5 & 30 & 40 & 30 \\
\hline E526 & metal mechanical grinder & before & 0.5 & all $^{\mathrm{c}}$ & all $^{\mathrm{c}}$ & $\mathrm{n} / \mathrm{a}$ \\
\hline E529 & metal mechanical grinder & before & $3-5$ & 30 & 30 & 30 \\
\hline E540 & metal mechanical grinder & before & 0.5 & all $^{\mathrm{c}}$ & all $^{\mathrm{C}}$ & $\mathrm{n} / \mathrm{a}$ \\
\hline E530 & mortar \& pestle & before & 0.5 & 5 & 5 & 5 \\
\hline E531 & mortar \& pestle & after & 0.25 & 5 & 5 & 5 \\
\hline E533 & mortar \& pestle & before & 1 to 2.2 & $\mathrm{n} / \mathrm{a}$ & $\mathrm{n} / \mathrm{a}$ & $\mathrm{n} / \mathrm{a}$ \\
\hline E534 & mortar \& pestle & before & 0.5 & all $^{\mathrm{C}}$ & all $^{\mathrm{C}}$ & all $^{\mathrm{c}}$ \\
\hline E537 & mortar \& pestle & before & 0.5 & 30 & 30 & 30 \\
\hline E538 & mortar \& pestle & before & $\mathrm{n} / \mathrm{a}$ & 1 & 1 & $\mathrm{n} / \mathrm{a}$ \\
\hline E539 & mortar \& pestle & before & 0.25 & 10 & 10 & 10 \\
\hline E541 & mortar \& pestle & before & 1 & 30 & 30 & 30 \\
\hline $\begin{array}{l}\text { E543 } \\
\text { (NIST) }\end{array}$ & mortar \& pestle & before & 0.5 & 30 & 30 & 30 \\
\hline E502 & $\begin{array}{c}\text { non-metal } \\
\text { mechanical grinder }\end{array}$ & before & 2 & 60 & 60 & 60 \\
\hline E513 & hammer & before & 1 & all & all & all \\
\hline E515 & hand & before & 0.5 & n/a & $\mathrm{n} / \mathrm{a}$ & $\mathrm{n} / \mathrm{a}$ \\
\hline
\end{tabular}

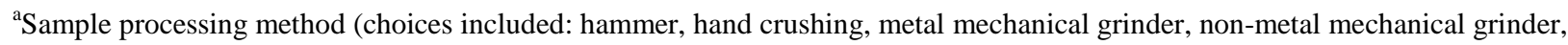
and mortar and pestle)

${ }^{\mathrm{b}}$ Stage when processing of the sample was performed, i.e., before or after subsampling for analysis

${ }^{\mathrm{c}}$ All of the original sample was used for processing (i.e., grinding, crushing, etc.) into subsamples for analysis. This includes labs which used all material from one package or combined all material from both packages. 
Table 3. Mean and standard deviation of the three sample results for Ca, Fe and Zn mass fractions (mg/kg) in each of the six cereals (A-F).

\begin{tabular}{|c|c|c|c|c|c|c|c|c|c|c|c|c|}
\hline \multirow{2}{*}{$\frac{\text { Ca }}{\text { Lab Code }}$} & \multicolumn{2}{|c|}{$\begin{array}{c}\text { Cereal A: } \\
\text { Wheat Ground } \\
\text { (SRM 3233) }\end{array}$} & \multicolumn{2}{|c|}{$\begin{array}{c}\text { Cereal B: } \\
\text { Wheat Ground }\end{array}$} & \multicolumn{2}{|c|}{$\begin{array}{c}\text { Cereal C: } \\
\text { Wheat Flake }\end{array}$} & \multicolumn{2}{|c|}{$\begin{array}{l}\text { Cereal D: } \\
\text { Rice Ground }\end{array}$} & \multicolumn{2}{|c|}{$\begin{array}{l}\text { Cereal E: } \\
\text { Rice Flake }\end{array}$} & \multicolumn{2}{|c|}{$\begin{array}{l}\text { Cereal F: } \\
\text { Wheat/Rice Crushed }\end{array}$} \\
\hline & Mean & SD & Mean & SD & Mean & SD & Mean & SD & Mean & SD & Mean & SD \\
\hline $\begin{array}{c}\text { E543 } \\
\text { (NIST) }\end{array}$ & 37248 & 336 & 34617 & 162 & 34502 & 496 & 282.3 & 10.5 & 279.4 & 4.8 & 21131 & 920 \\
\hline E502 & 37334 & 203 & 35756 & 262 & 35628 & 409 & 195.9 & 1.0 & 196.5 & 5.0 & 18259 & 171 \\
\hline E509 & 35100 & 854 & 34000 & $\mathrm{n} / \mathrm{a}$ & 33800 & 721 & 196.7 & 11.5 & 206.7 & 5.8 & 16700 & 1212 \\
\hline E510 & 32853 & 648 & 32010 & 331 & 30737 & 786 & 195.3 & 7.4 & 232.6 & 43.2 & 16297 & 346 \\
\hline E511 & 36633 & 1115 & 34433 & 924 & 34833 & 1012 & 196.0 & 6.0 & 198.7 & 7.6 & 17700 & 600 \\
\hline E513 & 37000 & 1000 & 34000 & 1000 & 34667 & 1155 & 180.0 & $\mathrm{n} / \mathrm{a}$ & 180.0 & $\mathrm{n} / \mathrm{a}$ & 16667 & 577 \\
\hline E515 & 35319 & 306 & 33266 & 384 & 34156 & 699 & 233.3 & 2.3 & 238.3 & 9.9 & 13128 & 1103 \\
\hline E520 & 41728 & 565 & 38718 & 223 & 38892 & 654 & 171.3 & 3.5 & 161.3 & 5.5 & 16120 & 538 \\
\hline E522 & 37253 & 338 & 35070 & 306 & 34858 & 455 & 144.7 & 3.5 & 146.3 & 2.1 & 17255 & 304 \\
\hline E526 & 36183 & 58 & 34417 & 126 & 34783 & 76 & 183.3 & 5.8 & 190.0 & $\mathrm{n} / \mathrm{a}$ & 16433 & 176 \\
\hline E529 & 38820 & 272 & 34707 & 353 & 37047 & 259 & 201.1 & 44.7 & 229.6 & 35.8 & 18417 & 1500 \\
\hline E531 & 38907 & 3290 & 37237 & 2622 & 37867 & 2137 & 263.3 & 23.1 & 213.3 & 23.1 & 18013 & 1213 \\
\hline E533 & 37533 & 252 & 35100 & 173 & 35433 & 723 & 233.3 & 11.5 & 216.7 & 5.8 & 16400 & 2805 \\
\hline E534 & 39700 & 529 & 37000 & 1308 & 37367 & 971 & 224.0 & 18.7 & 216.7 & 12.7 & 20667 & 1305 \\
\hline E537 & 40618 & 969 & 34166 & 782 & 37849 & 756 & 176.3 & 7.5 & 170.7 & 2.5 & 17364 & 228 \\
\hline E538 & 36602 & 1529 & 34323 & 1440 & 34974 & 1485 & 1247.9 & 314.3 & 960.3 & 435.3 & 19439 & 1760 \\
\hline E539 & 36700 & 458 & 34933 & 987 & 35500 & 608 & 154.3 & 17.0 & 390.0 & 91.3 & 19733 & 1436 \\
\hline E540 & 37133 & 379 & 34233 & 252 & 34800 & 346 & 460.0 & 20.0 & 440.0 & 10.0 & 18267 & 153 \\
\hline E541 & 40242 & 939 & 37811 & 297 & 39917 & 526 & 235.0 & 39.6 & 294.7 & 62.9 & 25932 & 5254 \\
\hline
\end{tabular}


Table 3 (cont'd). Mean and standard deviation of the three sample results for Ca, Fe and Zn mass fractions (mg/kg) in each of the six cereals (A-F).

\begin{tabular}{|c|c|c|c|c|c|c|c|c|c|c|c|c|}
\hline \multirow{2}{*}{$\frac{\text { Fe }}{\text { Lab Code }}$} & \multicolumn{2}{|c|}{$\begin{array}{c}\text { Cereal A: } \\
\text { Wheat Ground } \\
\text { (SRM 3233) }\end{array}$} & \multicolumn{2}{|c|}{$\begin{array}{c}\text { Cereal B: } \\
\text { Wheat Ground }\end{array}$} & \multicolumn{2}{|c|}{$\begin{array}{c}\text { Cereal C: } \\
\text { Wheat Flake }\end{array}$} & \multicolumn{2}{|c|}{$\begin{array}{l}\text { Cereal D: } \\
\text { Rice Ground }\end{array}$} & \multicolumn{2}{|c|}{$\begin{array}{l}\text { Cereal E: } \\
\text { Rice Flake }\end{array}$} & \multicolumn{2}{|c|}{$\begin{array}{l}\text { Cereal F: } \\
\text { Wheat/Rice Crushed }\end{array}$} \\
\hline & Mean & SD & Mean & SD & Mean & SD & Mean & SD & Mean & SD & Mean & SD \\
\hline $\begin{array}{c}\text { E543 } \\
\text { (NIST) }\end{array}$ & 785.3 & 10.1 & 844.7 & 21.4 & 838.5 & 7.3 & 524.0 & 15.7 & 533.7 & 17.2 & 738.3 & 10.1 \\
\hline E502 & 700.3 & 8.0 & 704.5 & 34.0 & 771.1 & 20.6 & 489.4 & 10.8 & 494.2 & 9.9 & 610.3 & 14.3 \\
\hline E509 & 720.0 & 26.5 & 786.7 & 25.2 & 820.0 & 20.0 & 453.3 & 5.8 & 426.7 & 30.6 & 603.3 & 15.3 \\
\hline E510 & 682.8 & 9.4 & 731.7 & 14.1 & 666.9 & 96.0 & 459.5 & 24.5 & 384.1 & 32.6 & 499.8 & 24.5 \\
\hline E511 & 747.0 & 25.1 & 805.7 & 23.0 & 818.3 & 29.4 & 516.3 & 12.4 & 506.0 & 8.0 & 654.0 & 22.0 \\
\hline E513 & 743.3 & 5.8 & 773.3 & 5.8 & 800.0 & 10.0 & 513.3 & 11.5 & 500.0 & $\mathrm{n} / \mathrm{a}$ & 660.0 & 10.0 \\
\hline E515 & 805.0 & 14.4 & 899.3 & 11.9 & 908.3 & 15.0 & 472.3 & 13.1 & 487.0 & 35.0 & 622.7 & 9.7 \\
\hline E520 & 860.7 & 21.1 & 912.0 & 7.0 & 937.7 & 13.1 & 572.0 & 11.5 & 577.0 & 15.5 & 703.0 & 9.2 \\
\hline E522 & 744.0 & 10.8 & 786.7 & 42.7 & 845.0 & 20.9 & 492.3 & 7.6 & 490.3 & 11.0 & 652.7 & 5.9 \\
\hline E526 & 747.0 & 5.8 & 826.0 & 1.7 & 847.7 & 42.6 & 506.7 & 7.2 & 501.3 & 4.7 & 667.0 & 6.2 \\
\hline E529 & $\mathrm{n} / \mathrm{d}$ & $\mathrm{n} / \mathrm{d}$ & $\mathrm{n} / \mathrm{d}$ & $\mathrm{n} / \mathrm{d}$ & $\mathrm{n} / \mathrm{d}$ & $\mathrm{n} / \mathrm{d}$ & $\mathrm{n} / \mathrm{d}$ & $\mathrm{n} / \mathrm{d}$ & $\mathrm{n} / \mathrm{d}$ & $\mathrm{n} / \mathrm{d}$ & $\mathrm{n} / \mathrm{d}$ & $\mathrm{n} / \mathrm{d}$ \\
\hline E530 & 374.9 & 35.5 & 697.0 & 64.6 & 438.7 & 50.3 & 461.0 & 41.9 & 331.7 & 30.8 & 346.7 & 69.0 \\
\hline E531 & 773.3 & 28.9 & 823.3 & 87.4 & 816.7 & 11.5 & 513.3 & 20.8 & 500.0 & 60.8 & 706.7 & 35.1 \\
\hline E533 & 690.0 & 20.0 & 753.3 & 28.9 & 793.3 & 5.8 & 460.0 & 10.0 & 466.7 & 11.5 & 583.3 & 32.1 \\
\hline E534 & 849.0 & 32.4 & 880.0 & 29.6 & 900.3 & 11.0 & 539.7 & 16.9 & 536.7 & 18.8 & 730.0 & 25.5 \\
\hline E537 & 716.3 & 7.5 & 782.7 & 19.4 & 746.0 & 14.0 & 517.0 & 8.9 & 513.3 & 4.9 & 627.3 & 16.9 \\
\hline E538 & 476.7 & 78.6 & 555.9 & 106.5 & 560.6 & 109.8 & 440.5 & 54.1 & 459.4 & 26.2 & 523.6 & 49.2 \\
\hline E539 & 782.0 & 11.4 & 855.0 & 14.5 & 849.7 & 40.1 & 513.3 & 62.3 & 570.7 & 34.8 & 733.7 & 13.5 \\
\hline E540 & 790.0 & 26.5 & 846.7 & 15.3 & 856.7 & 11.5 & 556.7 & 11.5 & 543.3 & 5.8 & 706.7 & 15.3 \\
\hline E541 & 706.3 & 7.1 & 779.0 & 7.5 & 800.0 & 2.0 & 508.3 & 13.1 & 501.3 & 11.6 & 685.3 & 7.4 \\
\hline
\end{tabular}


Table 3 (cont'd). Mean and standard deviation of the three sample results for Ca, Fe and Zn mass fractions (mg/kg) in each of the six cereals (A-F).

\begin{tabular}{|c|c|c|c|c|c|c|c|c|c|c|c|c|}
\hline \multirow{2}{*}{$\begin{array}{c}\text { Zn } \\
\text { Lab Code }\end{array}$} & \multicolumn{2}{|c|}{$\begin{array}{l}\text { Cereal A: } \\
\text { Wheat Ground } \\
\text { (SRM 3233) }\end{array}$} & \multicolumn{2}{|c|}{$\begin{array}{c}\text { Cereal B: } \\
\text { Wheat Ground }\end{array}$} & \multicolumn{2}{|c|}{$\begin{array}{c}\text { Cereal C: } \\
\text { Wheat Flake }\end{array}$} & \multicolumn{2}{|c|}{$\begin{array}{c}\text { Cereal D: } \\
\text { Rice Ground }\end{array}$} & \multicolumn{2}{|c|}{$\begin{array}{l}\text { Cereal E: } \\
\text { Rice Flake }\end{array}$} & \multicolumn{2}{|c|}{$\begin{array}{l}\text { Cereal F: } \\
\text { Wheat/Rice Crushed }\end{array}$} \\
\hline & Mean & SD & Mean & SD & Mean & SD & Mean & SD & Mean & SD & Mean & SD \\
\hline $\begin{array}{c}\text { E543 } \\
\text { (NIST) }\end{array}$ & 625.7 & 1.8 & 694.4 & 4.8 & 695.6 & 6.1 & 21.1 & 1.3 & 21.5 & 0.6 & 448.3 & 18.7 \\
\hline E502 & 631.1 & 0.6 & 716.3 & 5.6 & 715.6 & 5.2 & 23.6 & 0.1 & 23.7 & 0.1 & 396.6 & 3.0 \\
\hline E509 & 546.7 & 11.5 & 610.0 & 10.0 & 820.0 & 20.0 & 19.3 & 0.6 & 19.3 & 0.6 & 313.3 & 15.3 \\
\hline E510 & 536.4 & 30.0 & 587.4 & 37.5 & 521.6 & 66.0 & 20.5 & 0.7 & 17.7 & 1.9 & 284.2 & 7.1 \\
\hline E511 & 609.0 & 18.5 & 675.0 & 17.3 & 688.3 & 22.2 & 20.2 & 0.6 & 20.2 & 0.6 & 357.3 & 4.7 \\
\hline E513 & 533.3 & 5.8 & 573.3 & 5.8 & 586.7 & 5.8 & 18.3 & 0.6 & 18.3 & 0.6 & 320.0 & 17.3 \\
\hline E515 & 648.3 & 3.2 & 723.3 & 21.0 & 756.0 & 13.1 & 26.0 & $\mathrm{n} / \mathrm{a}$ & 25.3 & 0.6 & 299.0 & 25.5 \\
\hline E520 & 675.3 & 6.8 & 730.0 & 5.0 & 743.3 & 10.4 & 7.9 & 1.0 & 7.2 & 0.9 & 316.7 & 12.5 \\
\hline E522 & 620.0 & 6.6 & 687.7 & 6.1 & 699.3 & 10.5 & 19.4 & $\mathrm{n} / \mathrm{a}$ & 19.6 & 0.1 & 357.7 & 3.5 \\
\hline E526 & 607.6 & 10.7 & 679.3 & 4.0 & 709.3 & 7.0 & 20.6 & 0.2 & 20.2 & 0.3 & 342.7 & 2.9 \\
\hline E529 & 587.7 & 6.1 & 686.0 & 7.0 & 681.7 & 6.5 & 13.5 & 3.3 & 19.0 & 0.1 & 366.3 & 23.6 \\
\hline E530 & 590.2 & 4.7 & 674.0 & 3.0 & 646.7 & 25.0 & 23.3 & 2.7 & 20.8 & 1.2 & 318.8 & 6.7 \\
\hline E531 & 676.7 & 41.6 & 793.3 & 40.4 & 683.3 & 55.1 & 20.0 & $\mathrm{n} / \mathrm{a}$ & 20.0 & $\mathrm{n} / \mathrm{a}$ & 426.7 & 20.8 \\
\hline E533 & 573.3 & 5.8 & 650.0 & 36.1 & 706.7 & 20.8 & 30.0 & $\mathrm{n} / \mathrm{a}$ & 30.0 & $\mathrm{n} / \mathrm{a}$ & 326.7 & 50.3 \\
\hline E534 & 581.7 & 3.5 & 622.3 & 15.9 & 601.0 & 16.4 & 16.9 & 0.9 & 16.7 & 0.6 & 358.0 & 31.0 \\
\hline E537 & 618.7 & 3.2 & 660.3 & 8.1 & 652.7 & 11.2 & 20.2 & 0.3 & 20.0 & 0.3 & 310.0 & 11.8 \\
\hline E538 & 577.1 & 22.7 & 619.6 & 17.4 & 627.5 & 28.4 & 20.6 & 0.7 & 20.4 & 1.3 & 391.6 & 33.7 \\
\hline E539 & 612.7 & 0.6 & 684.3 & 7.0 & 701.7 & 26.8 & 19.0 & 1.4 & 25.1 & 1.3 & 416.3 & 34.2 \\
\hline E540 & 653.3 & 11.5 & 706.7 & 5.8 & 713.3 & 5.8 & 38.3 & 1.5 & 40.7 & 4.9 & 416.7 & 5.8 \\
\hline E541 & 553.0 & 1.7 & 609.7 & 1.2 & 624.0 & 2.0 & 18.7 & 0.6 & 19.3 & 1.5 & 357.7 & 4.7 \\
\hline
\end{tabular}


Table 4. Summary of overall exercise laboratory results and relative standard deviation (\%RSD) for the triplicate measurement results for the eighteen cereal/nutrient combinations

\begin{tabular}{|c|c|c|c|c|c|}
\hline Cereal & Description & $\mathbf{N}^{*}$ & $\begin{array}{c}\text { Median }^{\dagger} \\
\text { mg/kg }\end{array}$ & $\begin{array}{l}\text { Median }^{\ddagger} \\
\text { RSD }\end{array}$ & $\begin{array}{c}\text { Range } \\
\text { RSD }\end{array}$ \\
\hline \multicolumn{6}{|c|}{$\mathbf{F e}$} \\
\hline A & $\begin{array}{c}\text { Wheat } \\
\text { "Superground" }\end{array}$ & 19 & 37248 & 1.3 & 0.2 to 8.5 \\
\hline B & Wheat Ground & 18 & 34617 & 1.0 & 0.4 to 7.0 \\
\hline $\mathrm{C}$ & Wheat Flake & 19 & 34974 & 2.0 & 0.2 to 5.6 \\
\hline $\mathrm{D}$ & Rice Ground & 18 & 196 & 4.3 & 0.5 to 25.2 \\
\hline $\mathrm{E}$ & Rice Flake & 17 & 216 & 3.8 & 1.4 to 45.3 \\
\hline $\mathrm{F}$ & $\begin{array}{l}\text { Wheat/Rice } \\
\text { Crushed }\end{array}$ & 19 & 17700 & 4.4 & 0.8 to 20.3 \\
\hline \multicolumn{6}{|c|}{ Ca } \\
\hline A & $\begin{array}{c}\text { Wheat } \\
\text { "Superground" }\end{array}$ & 19 & 744 & 1.8 & 0.8 to 16.5 \\
\hline B & Wheat Ground & 19 & 787 & 2.5 & 0.2 to 19.2 \\
\hline $\mathrm{C}$ & Wheat Flake & 19 & 818 & 1.9 & 0.3 to 19.6 \\
\hline $\mathrm{D}$ & Rice Ground & 19 & 508 & 2.4 & 1.3 to 12.3 \\
\hline $\mathrm{E}$ & Rice Flake & 18 & 500 & 3.0 & 0.9 to 12.2 \\
\hline $\mathrm{F}$ & $\begin{array}{l}\text { Wheat/Rice } \\
\text { Crushed }\end{array}$ & 19 & 654 & 2.3 & 0.9 to 19.9 \\
\hline \multicolumn{6}{|c|}{$\mathbf{Z n}$} \\
\hline A & $\begin{array}{c}\text { Wheat } \\
\text { "Superground" }\end{array}$ & 20 & 608 & 1.0 & 0.1 to 6.1 \\
\hline B & Wheat Ground & 20 & 677 & 1.0 & 0.2 to 6.4 \\
\hline $\mathrm{C}$ & Wheat Flake & 20 & 692 & 1.7 & 0.3 to 12.7 \\
\hline $\mathrm{D}$ & Rice Ground & 16 & 20 & 3.4 & 0.4 to 24.4 \\
\hline $\mathrm{E}$ & Rice Flake & 18 & 20 & 3.2 & 0.4 to 12.5 \\
\hline $\mathrm{F}$ & $\begin{array}{l}\text { Wheat/Rice } \\
\text { Crushed }\end{array}$ & 20 & 358 & 4.1 & 0.8 to 15.4 \\
\hline
\end{tabular}

$* \mathrm{~N}=$ total number of laboratories summarized

${ }^{\dagger}$ Median of laboratory mean values and ${ }^{\ddagger}$ median RSD of the three sample results (Table 3) 
Table 5. Summary of consistency statistics ( $h$ and $k$ ) that exceeded the critical value at $5 \%$ significance level.

\begin{tabular}{|c|}
\hline Lab Code \\
\hline E543(NIST) \\
\hline E502 \\
\hline E509 \\
E510 \\
\hline E511 \\
\hline E513 \\
\hline E515 \\
\hline E520 \\
\hline E522 \\
\hline E526 \\
\hline E529 \\
\hline E530 \\
\hline E531 \\
\hline E533 \\
\hline E534 \\
\hline E537 \\
\hline E538 \\
\hline E539 \\
\hline E540 \\
\hline E541 \\
\hline Cereal: \\
\hline Element: \\
\hline
\end{tabular}
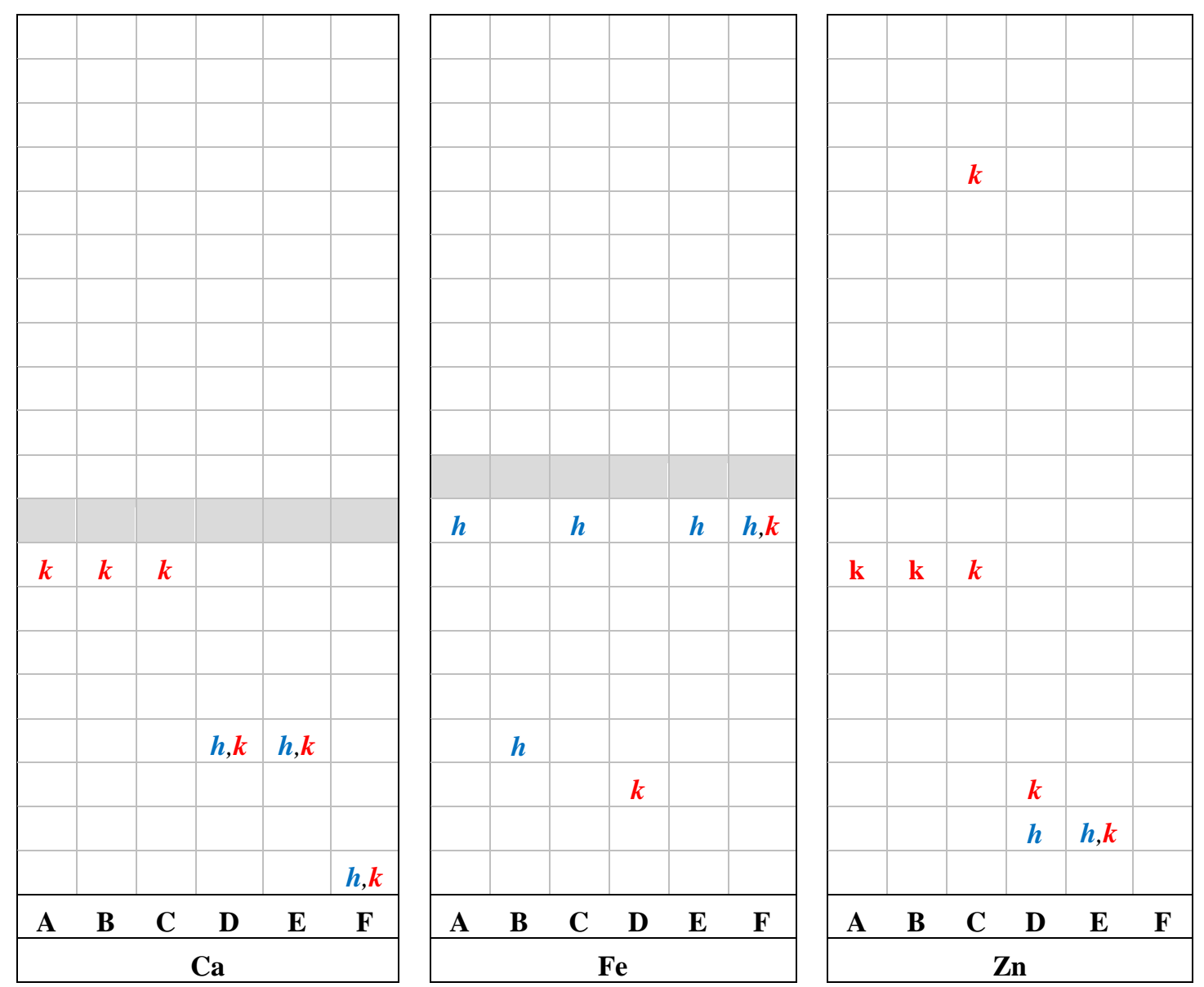

$\boldsymbol{h}=h$-consistency statistic $>$ critical value (significantly different with respect to between-laboratory variability). $\boldsymbol{k}=k$-consistency statistic $>$ critical value (significantly different with respect to within-laboratory variability). Gray-filled segments denote non-participation. 


\section{Appendices for NISTIR 7927}

An Interlaboratory Investigation of the Effect of Material Granularity and Sample Processing on Measurement Variability: Nutritional Elements in Cereals 


\section{APPENDIX A Sample Reporting Sheet}

\section{Sample preparation reporting sheet provided to participants}

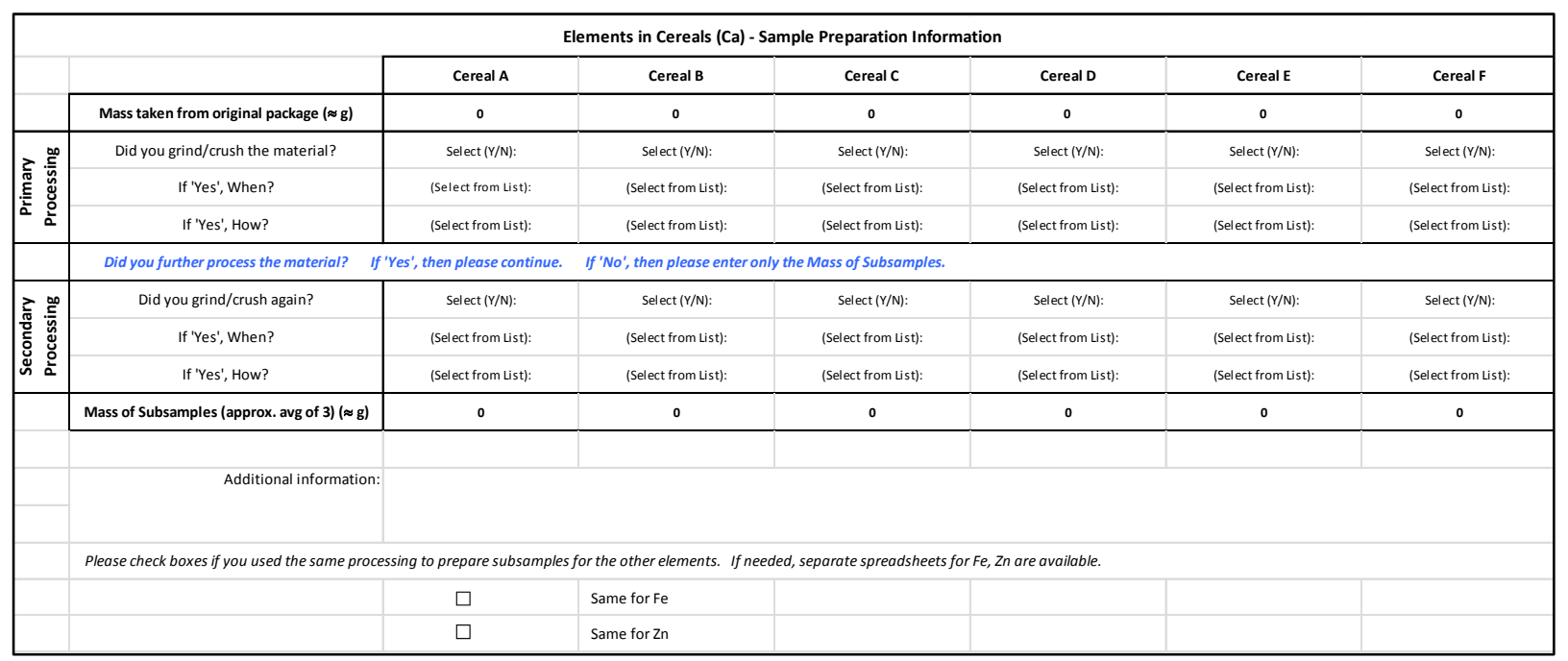

An exemplar sample preparation reporting sheet for Laboratory E543 (NIST) results:

\begin{tabular}{|c|c|c|c|c|c|c|c|}
\hline \multicolumn{8}{|c|}{ Elements in Cereals (Fe) - Sample Preparation Information } \\
\hline & & Cereal A & Cereal B & Cereal c & Cereal D & Cereal E & Cereal $\mathbf{F}$ \\
\hline & Mass taken from original package $(\approx \mathrm{g})$ & 0 & 0 & 30 & 0 & 30 & 30 \\
\hline \multirow{3}{*}{ 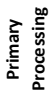 } & Did you grind/crush the material? & No & No & Yes & No & Yes & Yes \\
\hline & If 'Yes', When? & (Select from List): & (Select from List): & $\begin{array}{l}\text { Before replicates (processed } \\
\text { together) }\end{array}$ & (Select from List): & $\begin{array}{l}\text { Before replicates (processed } \\
\text { together) }\end{array}$ & $\begin{array}{l}\text { Before replicates (processed } \\
\text { together) }\end{array}$ \\
\hline & If 'Yes', How? & (Select from List): & (Select from List): & Manual-type Mortar \& Pestle & (Select from List): & Manual-type Mortar \& Pestle & Manual-type Mortar \& Pestle \\
\hline & \multicolumn{4}{|c|}{ Did you further process the material? If 'Yes', then please continue. If ' $N o$ ', then please enter only the Mass of Subsamples. } & & & \\
\hline \multirow{8}{*}{ 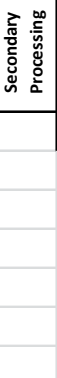 } & Did you grind/crush again? & Select $(Y / N):$ & Select $(\mathrm{Y} / \mathrm{N}):$ & No & Select $(\mathrm{Y} / \mathrm{N})$ : & No & No \\
\hline & If 'Yes', When? & (Select from List): & (Select from List): & (Select from List): & (Select from List): & (Select from List): & (Select from List): \\
\hline & If 'Yes', How? & (Select from List): & (Select from List): & (Select from List): & (Select from List): & (Select from List): & (Select from List): \\
\hline & Mass of Subsamples (approx. avg of 3) ( $\approx \mathrm{g}$ ) & 0 & 0 & 0.5 & 0 & 0.5 & 0.5 \\
\hline & Additional information: & & & & & & \\
\hline & \multicolumn{7}{|c|}{ Please check boxes if you used the same processing to prepare subsamples for the other elements. If needed, separate spreadsheets for Ca, Zn are available. } \\
\hline & & $\varpi$ & Same for $\mathrm{Ca}$ & & & & \\
\hline & & $\nabla$ & Same for $\mathrm{Zn}$ & & & & \\
\hline
\end{tabular}




\section{APPENDIX B Normal Probability Distribution Plots}

The following 18 plots are normal probability distributions for the individual mass fraction (or concentration) data for Ca, Fe and Zn $(\mathrm{mg} / \mathrm{kg})$ in the six materials (Cereals A-F). Probability plot correlation coefficients (PPCC) are provided with the $5 \%$ critical values $(\mathrm{CV})$. 

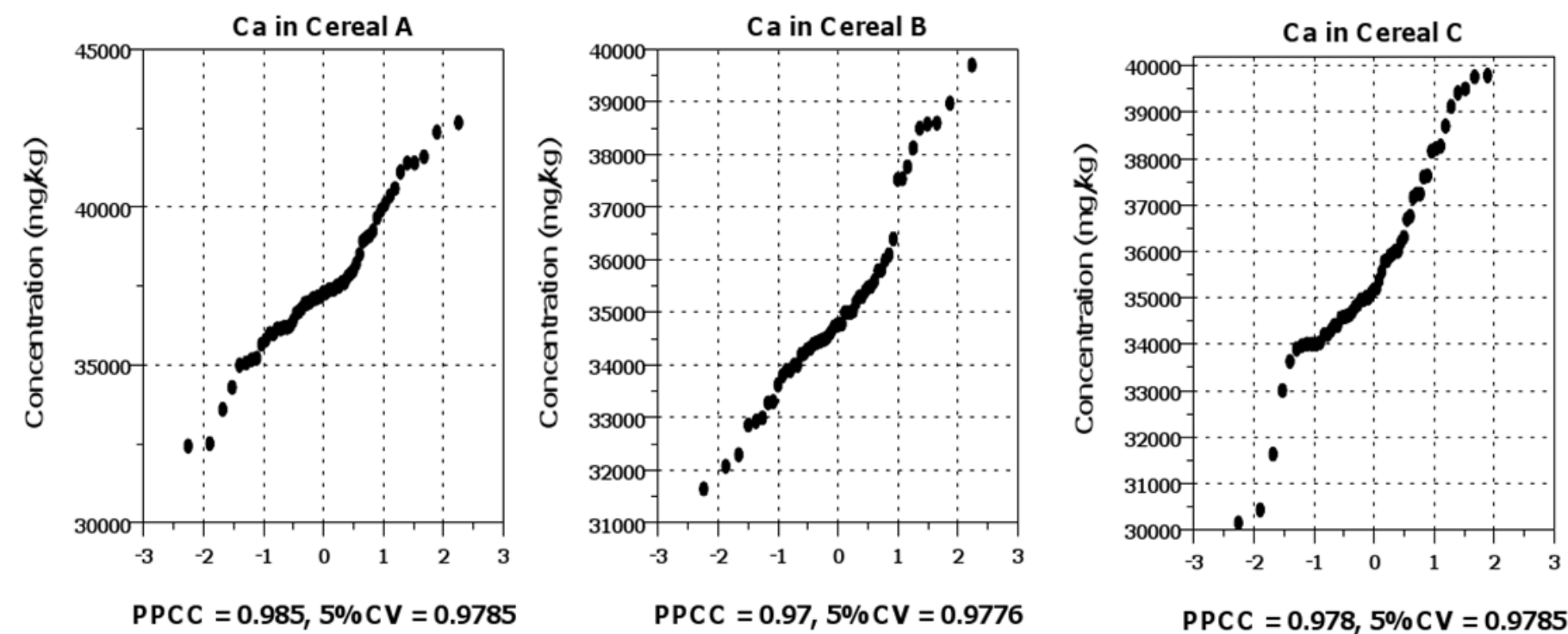

PPCC $=0.985,5 \% C V=0.9785$

PPCC $=0.978,5 \% C V=0.9785$
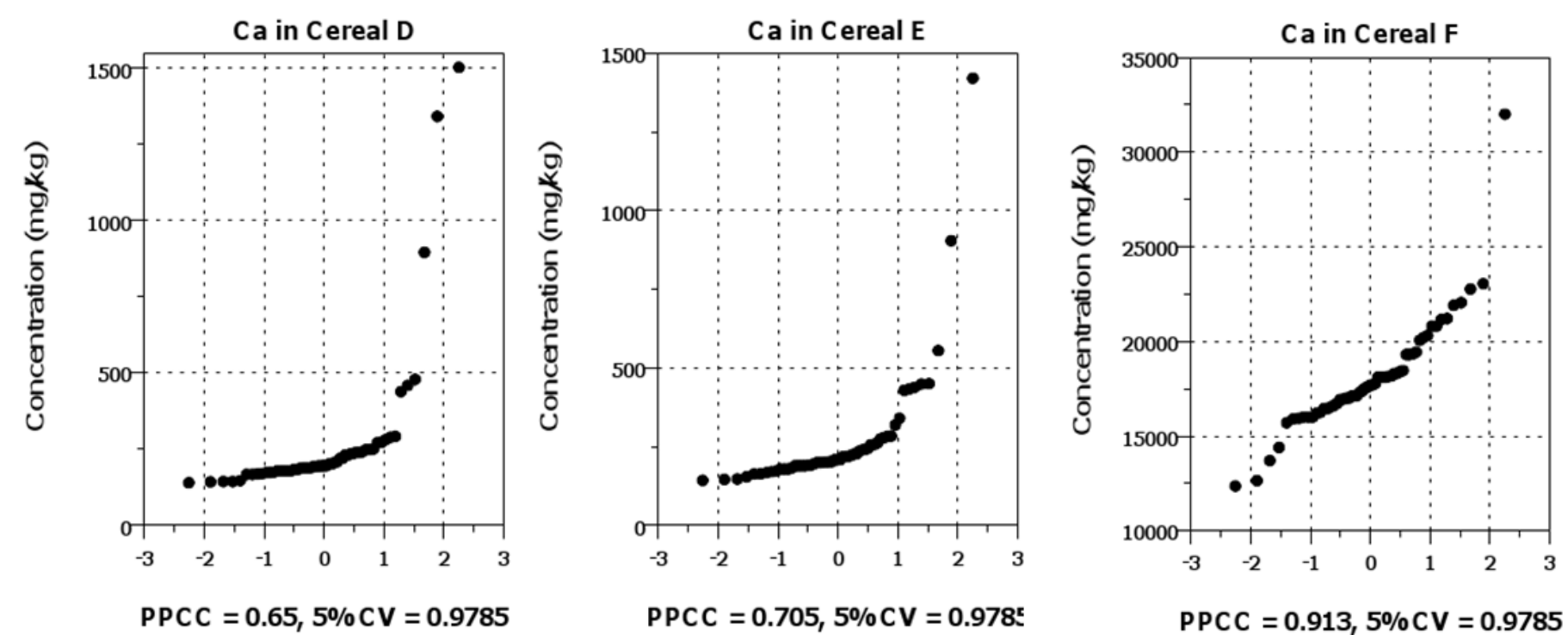

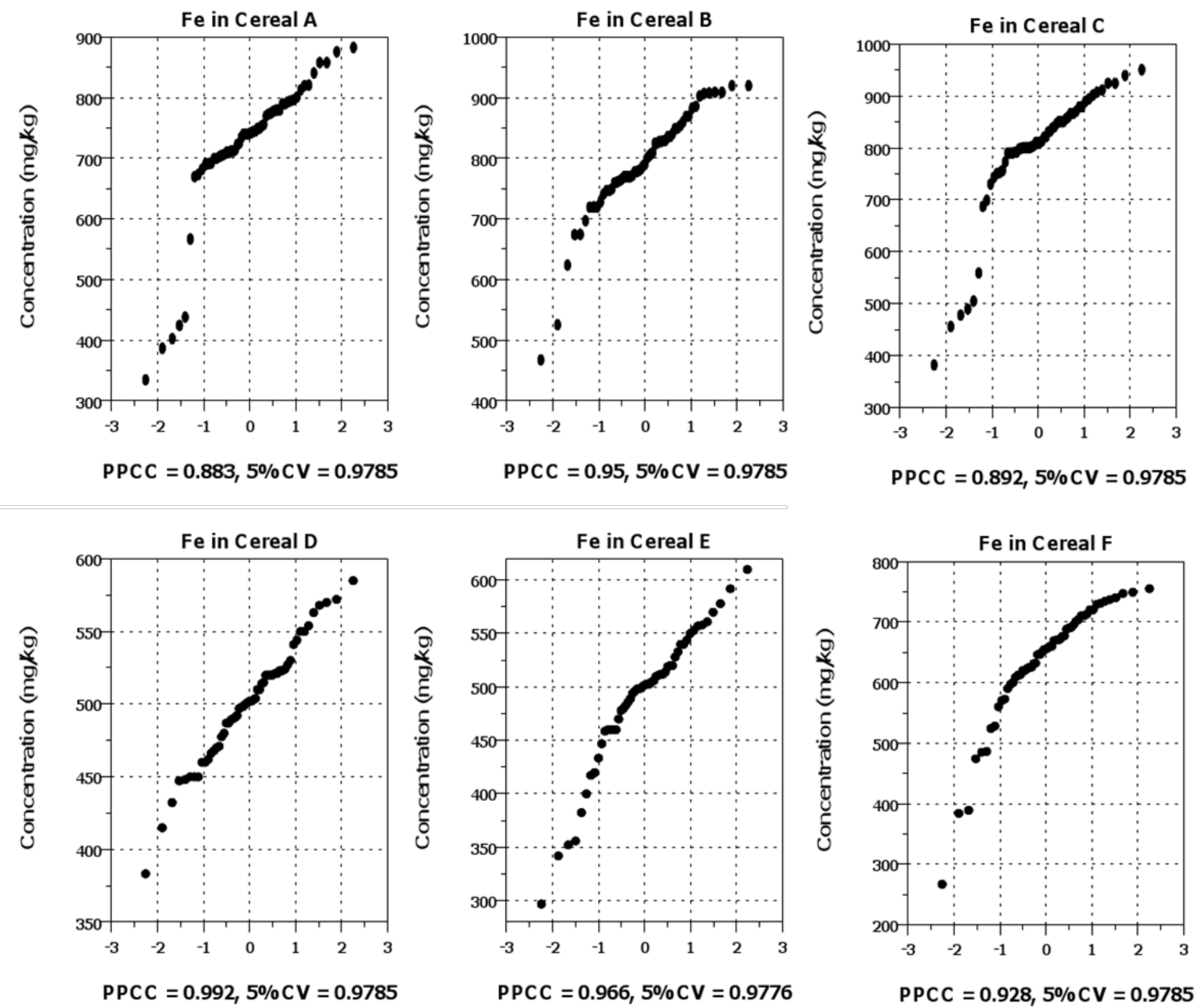

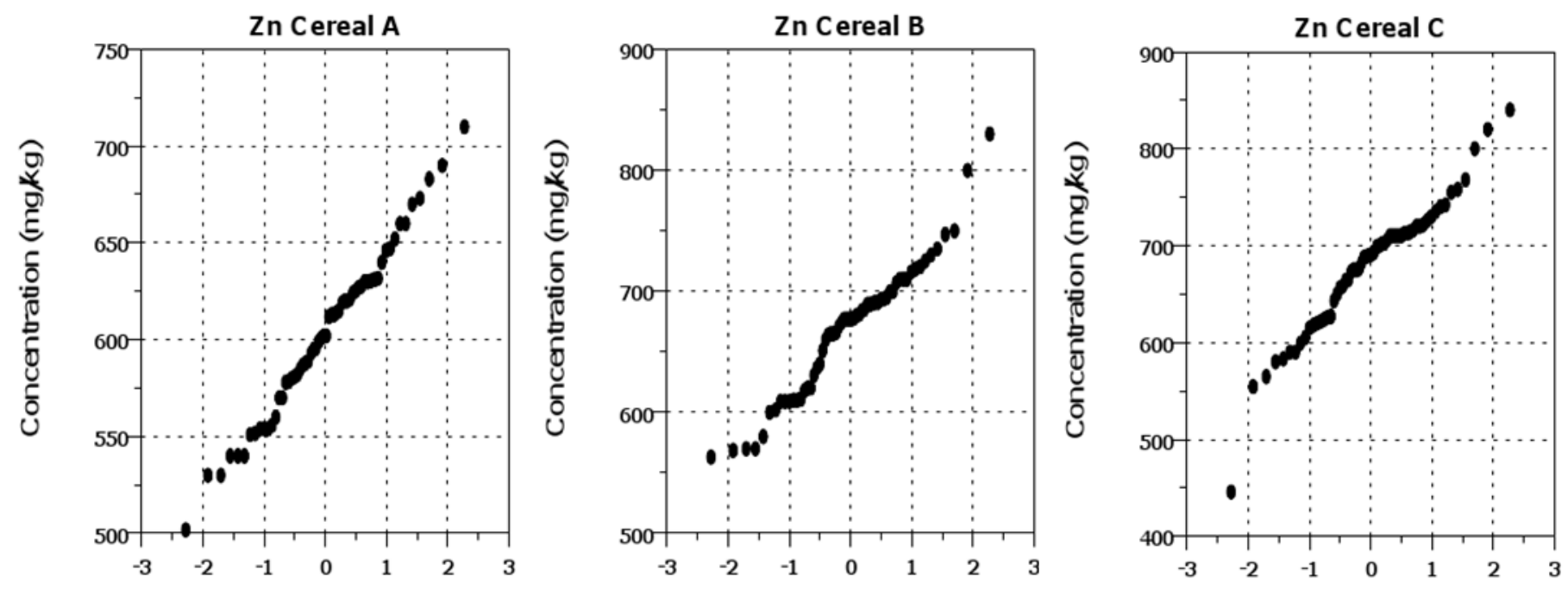

PPCC $=0.996,5 \% C V=0.9796$

PPCC $=0.982,5 \% C V=0.9796$

PPCC $=0.977,5 \% C V=0.9796$
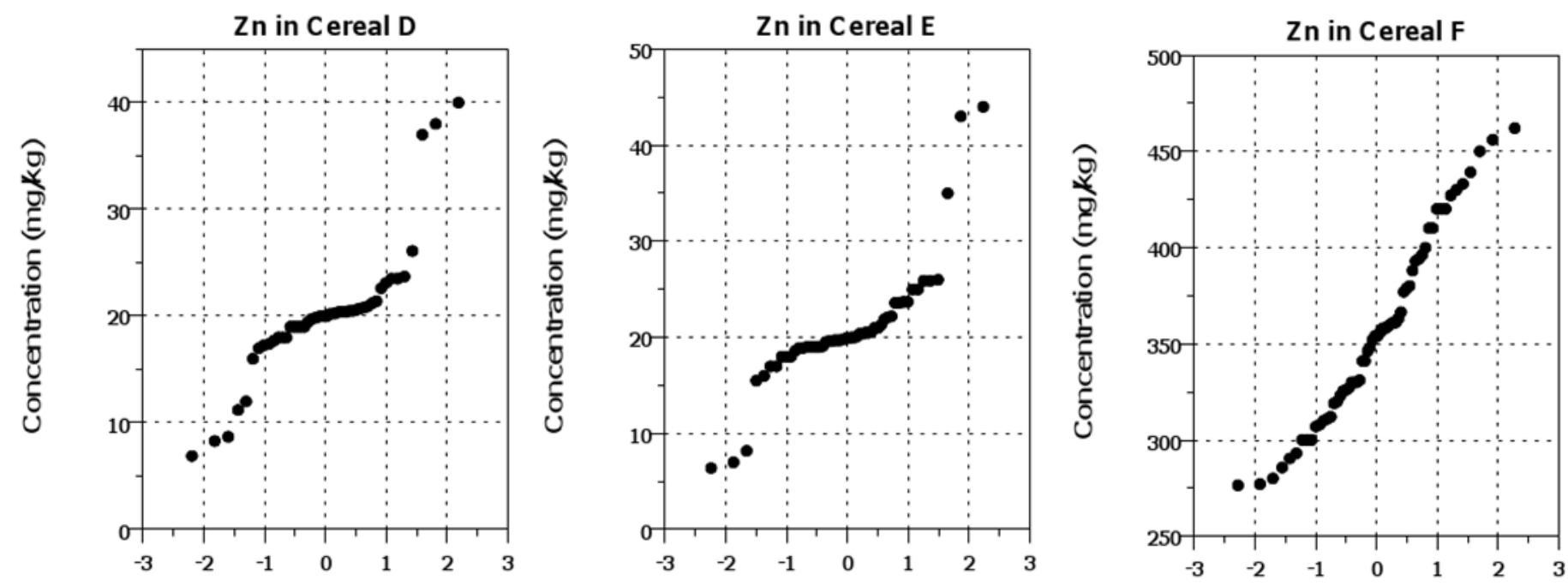

PPCC $=0.889,5 \% C V=0.9751$

PPCC $=0.987,5 \% C V=0.9796$ 


\section{APPENDIX C $h$-Consistency and $k$-Consistency Statistics Plots}

The following 6 sets of plots represent both the $h$-consistency statistics (between-laboratory) and $k$ consistency statistics (within-laboratory) for $\mathrm{Ca}, \mathrm{Fe}$ and $\mathrm{Zn}$ in Wheat (cereals A, B and C) and Ca, Fe and Zn in Rice (cereals D, E and F). The $5 \%$ significance levels (critical values) are denoted by a dashed horizontal line for each plot. Also provided with each set of $h$ - and $k$-plots are tabulated results from the corresponding interlaboratory analysis that includes the important determined E691parameters $(d, h, k)$. 

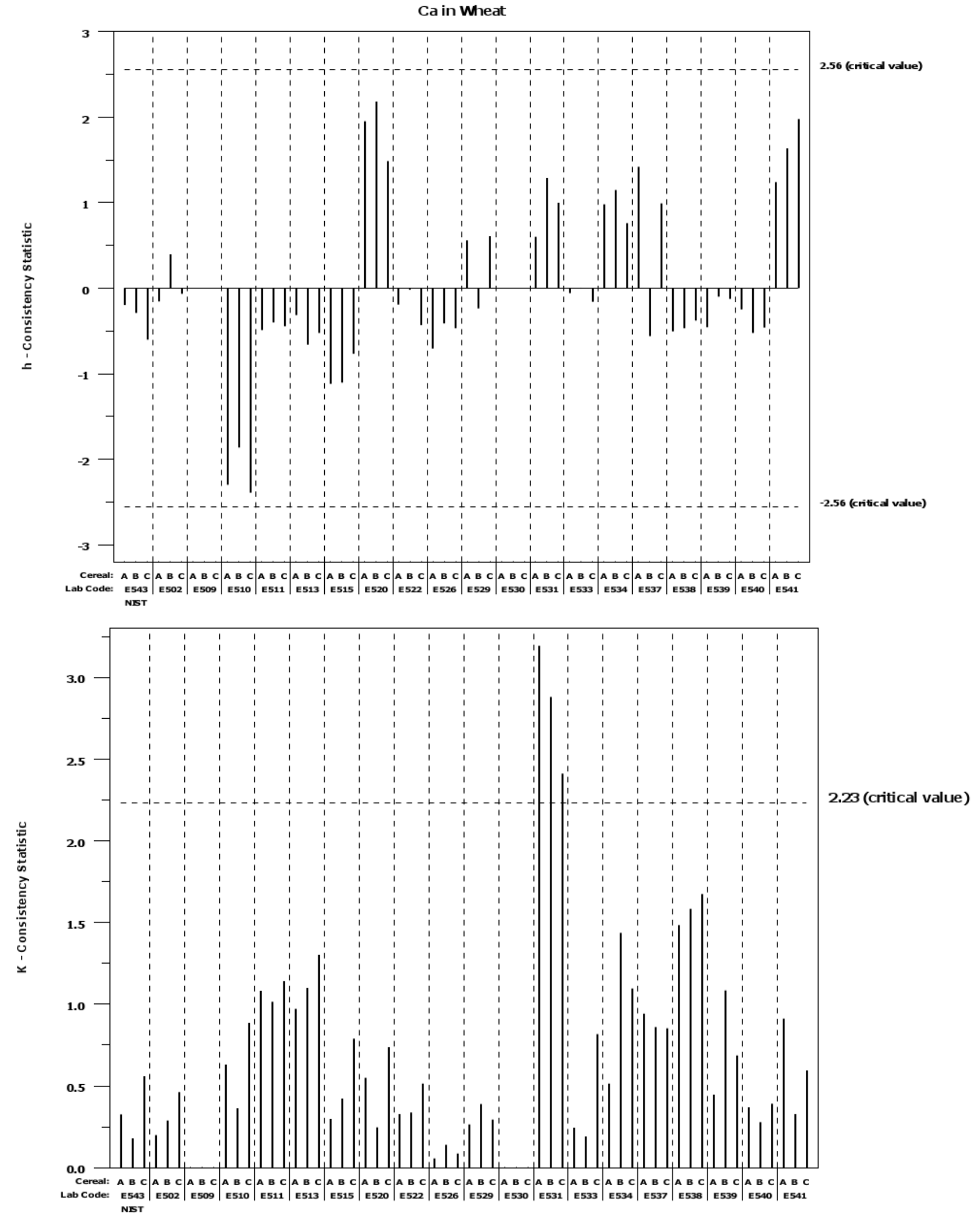
Ca

Initial Preparation of Test Result Data for Material: 1 (Cereal A)

\begin{tabular}{cccccc}
\hline $\begin{array}{c}\text { Laboratory } \\
\text { Number }\end{array}$ & $\begin{array}{c}\text { Cell } \\
\text { Mean }\end{array}$ & Cell & $\boldsymbol{d}$ & $\boldsymbol{h}$ & $\boldsymbol{k}$ \\
\hline 1 & 37247.67 & 335.50 & -408.30 & -0.20 & 0.33 \\
2 & 37334.00 & 203.27 & -321.96 & -0.15 & 0.20 \\
10 & 32853.33 & 647.87 & -4802.63 & -2.30 & 0.63 \\
11 & 36633.33 & 1115.05 & -1022.63 & -0.49 & 1.08 \\
13 & 37000.00 & 1000.00 & -655.96 & -0.31 & 0.97 \\
15 & 35319.00 & 305.78 & -2336.96 & -1.12 & 0.30 \\
20 & 41728.33 & 565.23 & 4072.37 & 1.95 & 0.55 \\
22 & 37252.67 & 337.59 & -403.30 & -0.19 & 0.33 \\
26 & 36183.33 & 57.74 & -1472.63 & -0.71 & 0.06 \\
29 & 38820.00 & 272.21 & 1164.04 & 0.56 & 0.26 \\
31 & 38906.67 & 3289.82 & 1250.70 & 0.60 & 3.19 \\
33 & 37533.33 & 251.66 & -122.63 & -0.06 & 0.24 \\
34 & 39700.00 & 529.15 & 2044.04 & 0.98 & 0.51 \\
37 & 40618.33 & 969.25 & 2962.37 & 1.42 & 0.94 \\
38 & 36601.67 & 1529.35 & -1054.30 & -0.51 & 1.48 \\
49 & 36700.00 & 458.26 & -955.96 & -0.46 & 0.44 \\
41 & 37133.33 & 378.59 & -522.63 & -0.25 & 0.37 \\
\hline
\end{tabular}

Average of cell averages $=37655.96296$

Standard Deviation of cell averages $=2087.27832$

Repeatability Standard Deviation $=1030.14276$

Reproducibility Standard Deviation $=2250.37631$

Ca

Initial Preparation of Test Result Data for Material: 2 (Cereal B)

\begin{tabular}{cccccc}
\hline $\begin{array}{c}\text { Laboratory } \\
\text { Number }\end{array}$ & $\begin{array}{c}\text { Cell } \\
\text { Mean }\end{array}$ & SD & $\boldsymbol{d}$ & $\boldsymbol{h}$ & $\boldsymbol{k}$ \\
\hline 1 & 34617.00 & 161.61 & -482.80 & -0.29 & 0.18 \\
2 & 35755.33 & 262.15 & 655.54 & 0.40 & 0.29 \\
10 & 32010.00 & 330.61 & -3089.80 & -1.86 & 0.36 \\
11 & 34433.33 & 923.76 & -666.46 & -0.40 & 1.01 \\
13 & 34000.00 & 1000.00 & -1099.80 & -0.66 & 1.10 \\
15 & 33265.67 & 383.67 & -1834.13 & -1.11 & 0.42 \\
20 & 38718.33 & 223.35 & 3618.54 & 2.18 & 0.25 \\
22 & 35069.67 & 305.90 & -30.13 & -0.02 & 0.34 \\
26 & 34416.67 & 125.83 & -683.13 & -0.41 & 0.14 \\
29 & 34706.67 & 353.03 & -393.13 & -0.24 & 0.39 \\
31 & 37236.67 & 2622.33 & 2136.87 & 1.29 & 2.88 \\
33 & 35100.00 & 173.21 & 0.20 & 0.00 & 0.19 \\
34 & 37000.00 & 1307.67 & 1900.20 & 1.15 & 1.44 \\
37 & 34166.00 & 781.88 & -933.80 & -0.56 & 0.86 \\
38 & 34323.00 & 1440.40 & -776.80 & -0.47 & 1.58 \\
39 & 34933.33 & 986.58 & -166.46 & -0.10 & 1.08 \\
40 & 34233.33 & 251.66 & -866.46 & -0.52 & 0.28 \\
41 & 37811.33 & 297.38 & 2711.54 & 1.64 & 0.33 \\
\hline
\end{tabular}

Average of cell averages $=35099.79630$

Standard Deviation of cell averages $=1657.89789$

Repeatability Standard Deviation $=910.32472$

Reproducibility Standard Deviation $=1816.88914$

Ca

Initial Preparation of Test Result Data for Material: 3 (Cereal C)

\begin{tabular}{cccccc}
\hline $\begin{array}{c}\text { Laboratory } \\
\text { Number }\end{array}$ & $\begin{array}{c}\text { Cell } \\
\text { Mean }\end{array}$ & $\begin{array}{c}\text { Cell } \\
\text { SD }\end{array}$ & $\boldsymbol{d}$ & $\boldsymbol{h}$ & $\boldsymbol{k}$ \\
\hline 1 & 34501.67 & 495.66 & -1265.54 & -0.60 & 0.56 \\
2 & 35628.33 & 409.16 & -138.87 & -0.07 & 0.46 \\
10 & 30736.67 & 786.21 & -5030.54 & -2.39 & 0.89 \\
11 & 34833.33 & 1011.60 & -933.87 & -0.44 & 1.14 \\
13 & 34666.67 & 1154.70 & -1100.54 & -0.52 & 1.30 \\
15 & 34156.00 & 698.67 & -1611.20 & -0.77 & 0.79 \\
20 & 38892.33 & 654.30 & 3125.13 & 1.49 & 0.74 \\
22 & 34857.67 & 454.57 & -909.54 & -0.43 & 0.51 \\
26 & 34783.33 & 76.38 & -983.87 & -0.47 & 0.09 \\
29 & 37046.67 & 259.29 & 1279.46 & 0.61 & 0.29 \\
31 & 37866.67 & 2137.32 & 2099.46 & 1.00 & 2.41 \\
33 & 35433.33 & 723.42 & -333.87 & -0.16 & 0.82 \\
34 & 37366.67 & 971.25 & 1599.46 & 0.76 & 1.10
\end{tabular}




\begin{tabular}{llllll}
37 & 37849.33 & 756.08 & 2082.13 & 0.99 & 0.85 \\
38 & 34973.67 & 1484.55 & -793.54 & -0.38 & 1.67 \\
39 & 35500.00 & 608.28 & -267.20 & -0.13 & 0.69 \\
40 & 34800.00 & 346.41 & -967.20 & -0.46 & 0.39 \\
41 & 39917.33 & 526.34 & 4150.13 & 1.97 & 0.59 \\
\hline
\end{tabular}

Average of cell averages $\quad=35767.20370$

Standard Deviation of cell averages $=2101.51320$

Repeatability Standard Deviation $=886.98024$

Reproducibility Standard Deviation $=2222.80162$

Ca-h

\begin{tabular}{|c|c|c|c|}
\hline \multirow[b]{2}{*}{ Laboratory } & \multicolumn{3}{|c|}{ Material } \\
\hline & 1 & 2 & 3 \\
\hline 1 & -0.20 & -0.29 & -0.60 \\
\hline 2 & -0.15 & 0.40 & -0.07 \\
\hline 10 & -2.30 & -1.86 & -2.39 \\
\hline 11 & -0.49 & -0.40 & -0.44 \\
\hline 13 & -0.31 & -0.66 & -0.52 \\
\hline 15 & -1.12 & -1.11 & -0.77 \\
\hline 20 & 1.95 & 2.18 & 1.49 \\
\hline 22 & -0.19 & -0.02 & -0.43 \\
\hline 26 & -0.71 & -0.41 & -0.47 \\
\hline 29 & 0.56 & -0.24 & 0.61 \\
\hline 31 & 0.60 & 1.29 & 1.00 \\
\hline 33 & -0.06 & 0.00 & -0.16 \\
\hline 34 & 0.98 & 1.15 & 0.76 \\
\hline 37 & 1.42 & -0.56 & 0.99 \\
\hline 38 & -0.51 & -0.47 & -0.38 \\
\hline 39 & -0.46 & -0.10 & -0.13 \\
\hline 40 & -0.25 & -0.52 & -0.46 \\
\hline 41 & 1.24 & 1.64 & 1.97 \\
\hline
\end{tabular}

Critical Value $=2.53000$

\begin{tabular}{cccc} 
Ca-k & \multicolumn{3}{l}{} \\
\hline Laboratory & 1 & Material & 3 \\
\hline 1 & 0.33 & 0.18 & 0.56 \\
2 & 0.20 & 0.29 & 0.46 \\
10 & 0.63 & 0.36 & 0.89 \\
11 & 1.08 & 1.01 & 1.14 \\
13 & 0.97 & 1.10 & 1.30 \\
15 & 0.30 & 0.42 & 0.79 \\
20 & 0.55 & 0.25 & 0.74 \\
22 & 0.33 & 0.34 & 0.51 \\
26 & 0.06 & 0.14 & 0.09 \\
29 & 0.26 & 0.39 & 0.29 \\
31 & 3.19 & 2.88 & 2.41 \\
33 & 0.24 & 0.19 & 0.82 \\
34 & 0.51 & 1.44 & 1.10 \\
37 & 0.94 & 0.86 & 0.85 \\
38 & 1.48 & 1.58 & 1.67 \\
39 & 0.44 & 1.08 & 0.69 \\
40 & 0.37 & 0.28 & 0.39 \\
41 & 0.91 & 0.33 & 0.59 \\
\hline
\end{tabular}

Critical Value $=\quad 2.20000$

Ca-Precision Statistics

\begin{tabular}{ccccccc}
\hline Material & Xbar & $\boldsymbol{s}_{\boldsymbol{x}}$ & $\boldsymbol{S}_{\boldsymbol{r}}$ & $\boldsymbol{S}_{\boldsymbol{R}}$ & $\boldsymbol{r}$ \\
\hline 1 & 37655.96 & 2087.28 & 1030.14 & 2250.38 & 2884.40 & 6301.05 \\
2 & 35099.80 & 1657.90 & 910.32 & 1816.89 & 2548.91 & 5087.29 \\
3 & 35767.20 & 2101.51 & 886.98 & 2222.80 & 2483.54 & 6223.84 \\
\hline
\end{tabular}



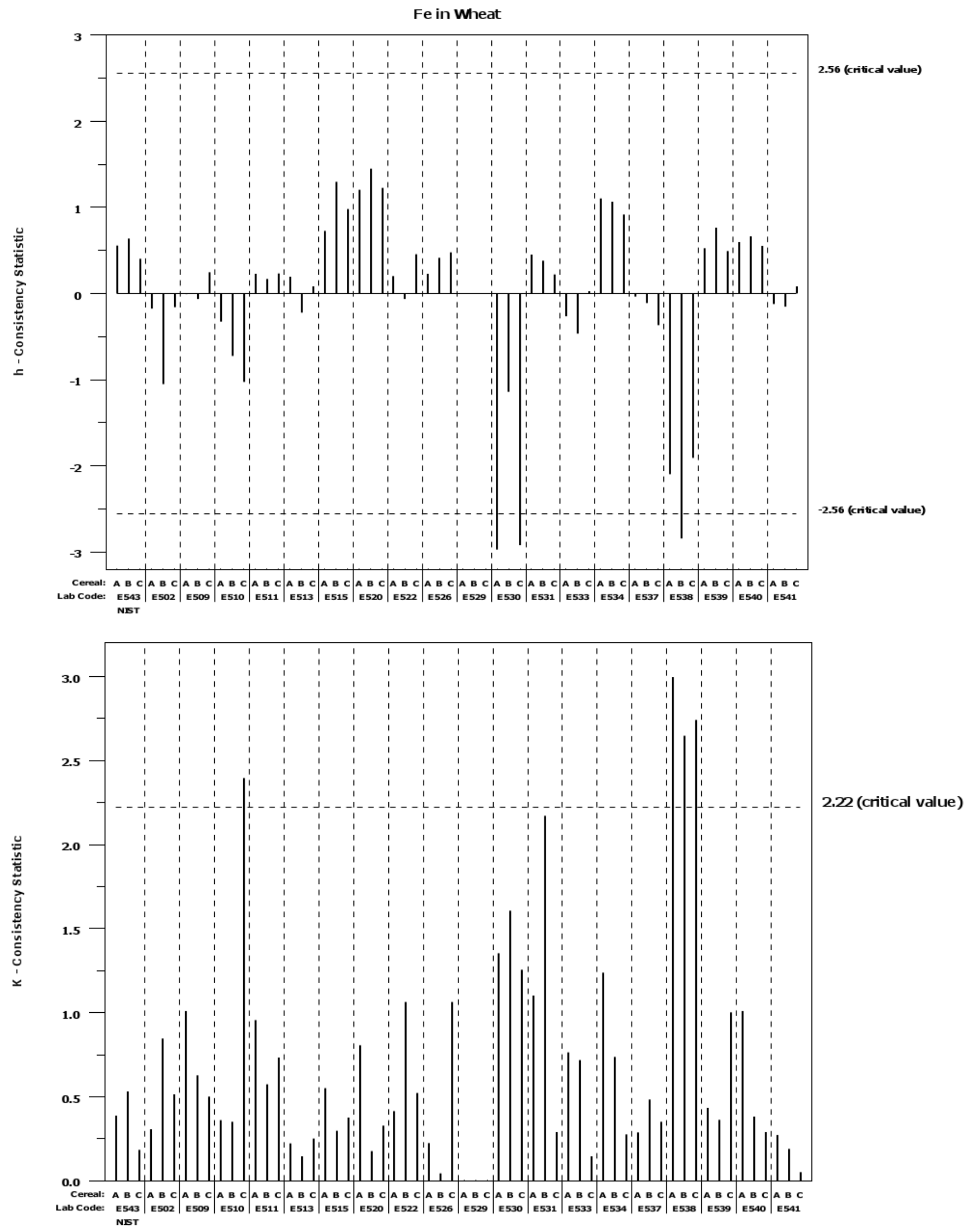
$\mathrm{Fe}$

Initial Preparation of Test Result Data for Material: 1 (Cereal A)

\begin{tabular}{cccccc}
\hline $\begin{array}{c}\text { Laboratory } \\
\text { Number }\end{array}$ & $\begin{array}{c}\text { Cell } \\
\text { Mean }\end{array}$ & $\begin{array}{c}\text { Cell } \\
\text { SD }\end{array}$ & $\boldsymbol{d}$ & $\boldsymbol{h}$ & $\boldsymbol{k}$ \\
\hline 1 & 785.33 & 10.12 & 64.60 & 0.56 & 0.39 \\
2 & 700.27 & 8.01 & -20.47 & -0.18 & 0.31 \\
9 & 720.00 & 26.46 & -0.74 & -0.01 & 1.01 \\
10 & 682.77 & 9.41 & -37.97 & -0.33 & 0.36 \\
11 & 747.00 & 25.06 & 26.26 & 0.23 & 0.96 \\
13 & 743.33 & 5.77 & 22.60 & 0.19 & 0.22 \\
15 & 805.00 & 14.42 & 84.26 & 0.72 & 0.55 \\
20 & 860.67 & 21.13 & 139.93 & 1.20 & 0.81 \\
22 & 744.00 & 10.82 & 23.26 & 0.20 & 0.41 \\
26 & 747.03 & 5.84 & 26.30 & 0.23 & 0.22 \\
30 & 374.93 & 35.47 & -345.80 & -2.97 & 1.35 \\
31 & 773.33 & 28.87 & 52.60 & 0.45 & 1.10 \\
33 & 690.00 & 20.00 & -30.74 & -0.26 & 0.76 \\
34 & 849.00 & 32.45 & 128.26 & 1.10 & 1.24 \\
37 & 716.33 & 7.51 & -4.40 & -0.04 & 0.29 \\
38 & 476.67 & 78.62 & -244.07 & -2.10 & 3.00 \\
39 & 782.00 & 11.36 & 61.26 & 0.53 & 0.43 \\
41 & 790.00 & 26.46 & 69.26 & 0.60
\end{tabular}

Average of cell averages $\quad=720.73684$

Standard Deviation of cell averages $=116.24638$

Repeatability Standard Deviation $=26.24053$

Reproducibility Standard Deviation $=118.20434$

Fe

Initial Preparation of Test Result Data for Material: 2 (Cereal B)

\begin{tabular}{cccccc}
\hline $\begin{array}{c}\text { Laboratory } \\
\text { Number }\end{array}$ & $\begin{array}{c}\text { Cell } \\
\text { Mean }\end{array}$ & $\begin{array}{c}\text { Cell } \\
\text { SD }\end{array}$ & $\boldsymbol{d}$ & $\boldsymbol{h}$ & $\boldsymbol{k}$ \\
\hline 1 & 844.67 & 21.36 & 52.91 & 0.64 & 0.53 \\
2 & 704.50 & 34.04 & -87.26 & -1.05 & 0.85 \\
9 & 786.67 & 25.17 & -5.09 & -0.06 & 0.63 \\
10 & 731.67 & 14.06 & -60.09 & -0.72 & 0.35 \\
11 & 805.67 & 23.03 & 13.91 & 0.17 & 0.57 \\
13 & 773.33 & 5.77 & -18.43 & -0.22 & 0.14 \\
15 & 899.33 & 11.93 & 107.57 & 1.30 & 0.30 \\
20 & 912.00 & 7.00 & 120.24 & 1.45 & 0.17 \\
22 & 786.67 & 42.74 & -5.09 & -0.06 & 1.06 \\
26 & 826.00 & 1.73 & 34.24 & 0.41 & 0.04 \\
30 & 697.00 & 64.65 & -94.76 & -1.14 & 1.61 \\
31 & 823.33 & 87.37 & 31.57 & 0.38 & 2.17 \\
33 & 753.33 & 28.87 & -38.43 & -0.46 & 0.72 \\
34 & 880.00 & 29.61 & 88.24 & 1.06 & 0.74 \\
37 & 782.67 & 19.43 & -9.09 & -0.11 & 0.48 \\
38 & 555.93 & 106.52 & -235.83 & -2.84 & 2.65 \\
39 & 855.00 & 14.53 & 63.24 & 0.76 & 0.36 \\
40 & 846.67 & 15.28 & 54.91 & 0.66 & 0.38 \\
41 & 779.00 & 7.55 & -12.76 & -0.15 & 0.19 \\
\hline
\end{tabular}

Average of cell averages $\quad=\quad 791.75965$

Standard Deviation of cell averages $=82.97406$

Repeatability Standard Deviation $=40.24783$

Reproducibility Standard Deviation $=89.24472$

Fe

Initial Preparation of Test Result Data for Material: 3 (Cereal C)

\begin{tabular}{cccccc}
\hline $\begin{array}{c}\text { Laboratory } \\
\text { Number }\end{array}$ & $\begin{array}{c}\text { Cell } \\
\text { Mean }\end{array}$ & $\begin{array}{c}\text { Cell } \\
\text { SD }\end{array}$ & $\boldsymbol{d}$ & $\boldsymbol{h}$ & $\boldsymbol{k}$ \\
\hline 1 & 838.53 & 7.29 & 48.25 & 0.40 & 0.18 \\
2 & 771.13 & 20.57 & -19.15 & -0.16 & 0.51 \\
9 & 820.00 & 20.00 & 29.71 & 0.25 & 0.50 \\
10 & 666.90 & 96.01 & -123.39 & -1.03 & 2.40 \\
11 & 818.33 & 29.37 & 28.05 & 0.23 & 0.73 \\
13 & 800.00 & 10.00 & 9.71 & 0.08 & 0.25 \\
15 & 908.33 & 15.04 & 118.05 & 0.98 & 0.38 \\
20 & 937.67 & 13.05 & 147.38 & 1.22 & 0.33 \\
22 & 845.00 & 20.88 & 54.71 & 0.45 & 0.52 \\
26 & 847.67 & 42.57 & 57.38 & 0.48 & 1.06 \\
30 & 438.67 & 50.29 & -351.62 & -2.92 & 1.25
\end{tabular}




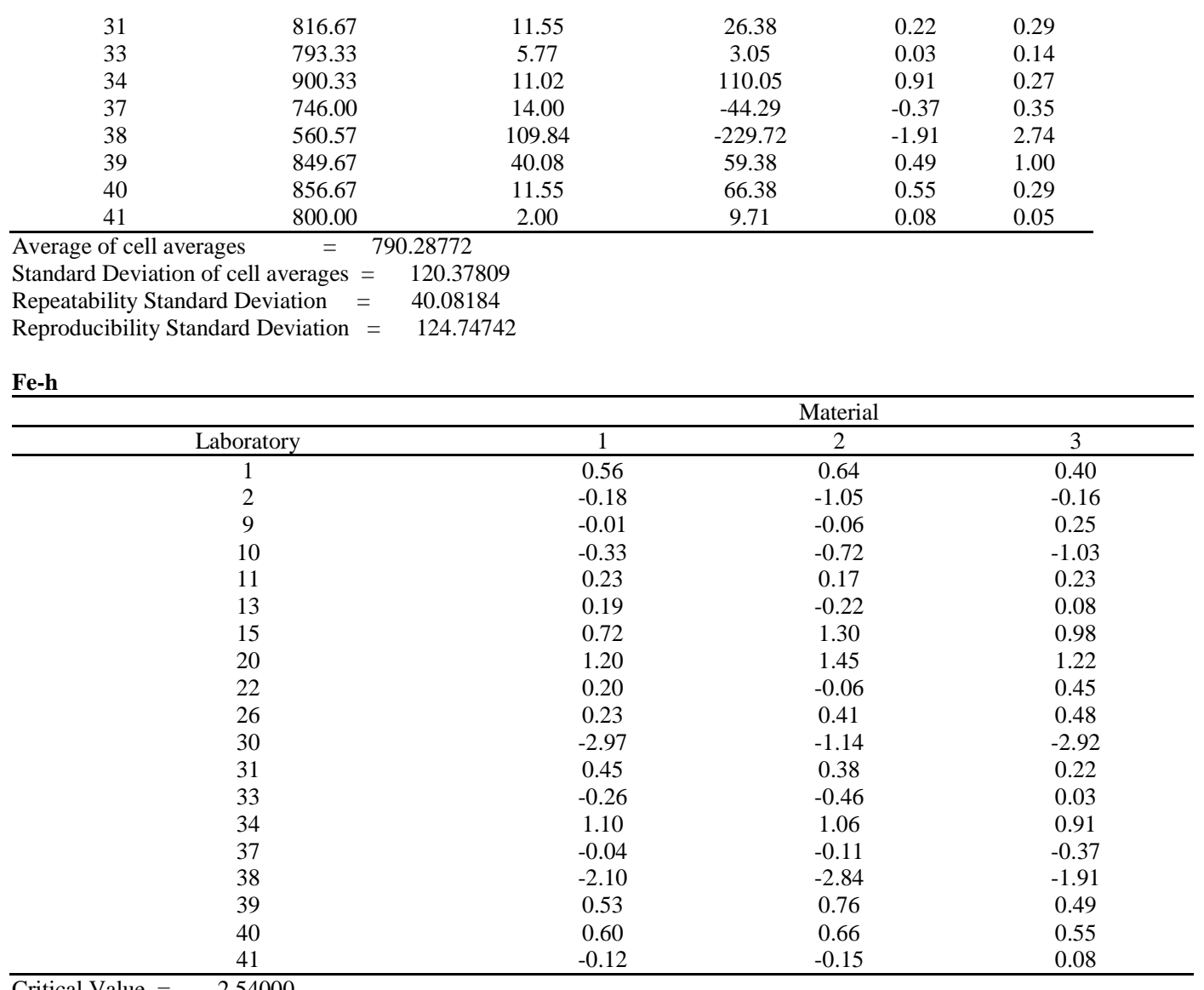

Critical Value $=\quad 2.54000$

Fe-k

\begin{tabular}{|c|c|c|c|}
\hline & \multicolumn{3}{|c|}{ Material } \\
\hline Laboratory & 1 & 2 & 3 \\
\hline 1 & 0.39 & 0.53 & 0.18 \\
\hline 2 & 0.31 & 0.85 & 0.51 \\
\hline 9 & 1.01 & 0.63 & 0.50 \\
\hline 10 & 0.36 & 0.35 & 2.40 \\
\hline 11 & 0.96 & 0.57 & 0.73 \\
\hline 13 & 0.22 & 0.14 & 0.25 \\
\hline 15 & 0.55 & 0.30 & 0.38 \\
\hline 20 & 0.81 & 0.17 & 0.33 \\
\hline 22 & 0.41 & 1.06 & 0.52 \\
\hline 26 & 0.22 & 0.04 & 1.06 \\
\hline 30 & 1.35 & 1.61 & 1.25 \\
\hline 31 & 1.10 & 2.17 & 0.29 \\
\hline 33 & 0.76 & 0.72 & 0.14 \\
\hline 34 & 1.24 & 0.74 & 0.27 \\
\hline 37 & 0.29 & 0.48 & 0.35 \\
\hline 38 & 3.00 & 2.65 & 2.74 \\
\hline 39 & 0.43 & 0.36 & 1.00 \\
\hline 40 & 1.01 & 0.38 & 0.29 \\
\hline 41 & 0.27 & 0.19 & 0.05 \\
\hline
\end{tabular}

Critical Value $=\quad 2.20000$

Fe-Precision Statistics

\begin{tabular}{crrrrrr}
\hline Material & Xbar & $\boldsymbol{S}_{\boldsymbol{X}}$ & $\boldsymbol{S}_{\boldsymbol{r}}$ & $\boldsymbol{S}_{\boldsymbol{R}}$ & $\boldsymbol{r}$ & $\boldsymbol{R}$ \\
\hline 1 & 720.74 & 116.25 & 26.24 & 118.20 & 73.47 & 330.97 \\
2 & 791.76 & 82.97 & 40.25 & 89.24 & 112.69 & 249.89 \\
3 & 790.29 & 120.38 & 40.08 & 124.75 & 112.23 & 349.29 \\
\hline
\end{tabular}



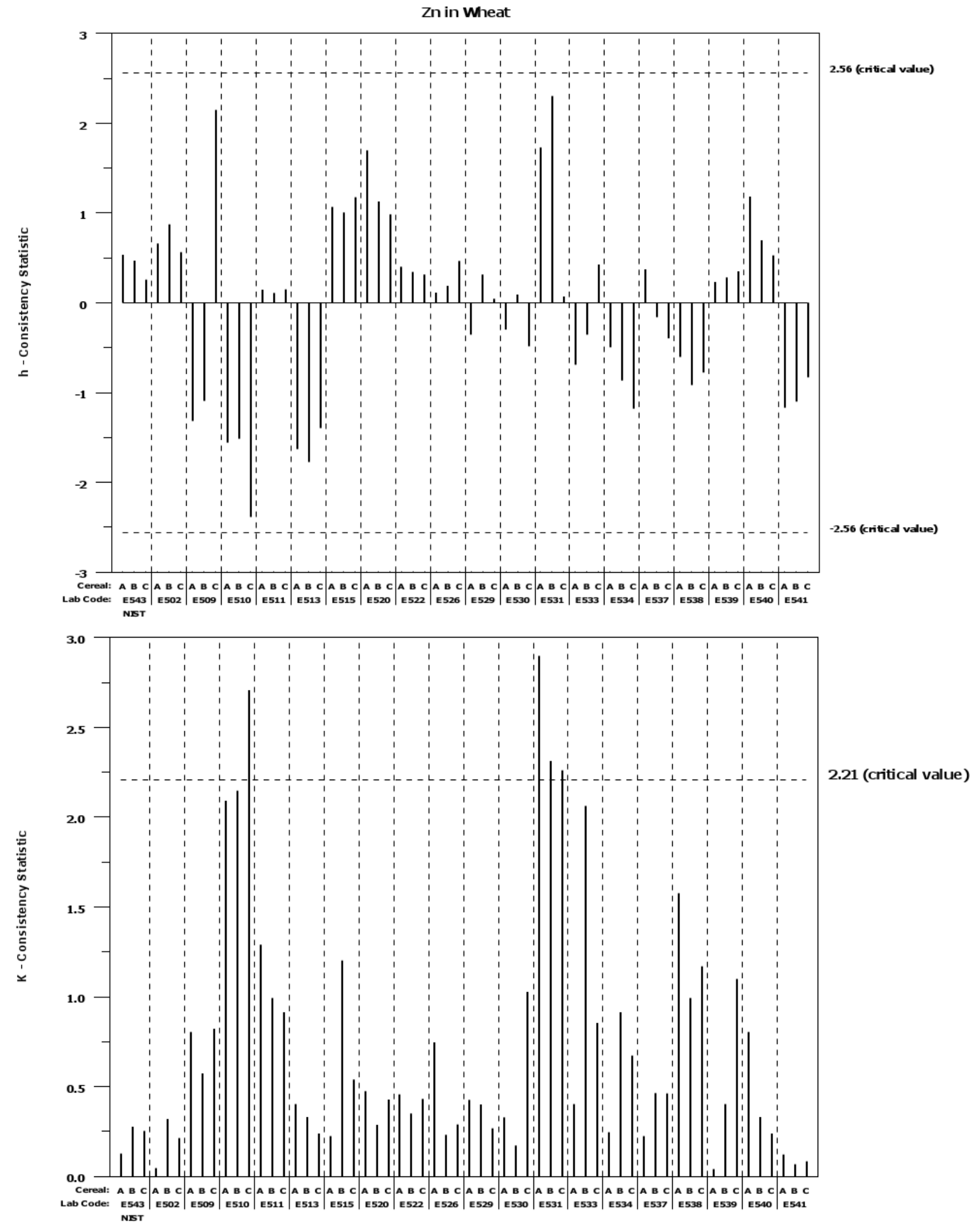
Zn

Initial Preparation of Test Result Data for Material: 1 (Cereal A)

\begin{tabular}{cccccc}
\hline $\begin{array}{c}\text { Laboratory } \\
\text { Number }\end{array}$ & $\begin{array}{c}\text { Cell } \\
\text { Mean }\end{array}$ & Cell & $\boldsymbol{d}$ & $\boldsymbol{h}$ & $\boldsymbol{k}$ \\
\hline 1 & 625.67 & 1.82 & 22.78 & 0.53 & 0.13 \\
2 & 631.07 & 0.65 & 28.18 & 0.66 & 0.05 \\
9 & 546.67 & 11.55 & -56.22 & -1.32 & 0.80 \\
10 & 536.40 & 30.04 & -66.49 & -1.56 & 2.09 \\
11 & 609.00 & 18.52 & 6.11 & 0.14 & 1.29 \\
13 & 533.33 & 5.77 & -69.56 & -1.63 & 0.40 \\
15 & 648.33 & 3.21 & 45.44 & 1.06 & 0.22 \\
20 & 675.33 & 6.81 & 72.44 & 1.70 & 0.47 \\
22 & 620.00 & 6.56 & 17.11 & 0.40 & 0.46 \\
26 & 607.63 & 10.72 & 4.74 & 0.11 & 0.75 \\
29 & 587.67 & 6.11 & -15.22 & -0.36 & 0.43 \\
30 & 590.20 & 4.70 & -12.69 & -0.30 & 0.33 \\
31 & 676.67 & 41.63 & 73.78 & 1.73 & 2.90 \\
33 & 573.33 & 5.77 & -29.56 & -0.69 & 0.40 \\
34 & 581.67 & 3.51 & -21.22 & -0.50 & 0.24 \\
37 & 618.67 & 3.21 & 15.78 & 0.37 & 0.22 \\
38 & 577.13 & 22.63 & -25.76 & -0.60 & 1.58 \\
39 & 612.67 & 0.58 & 9.78 & 0.23
\end{tabular}

Average of cell averages $=602.88833$

Standard Deviation of cell averages $=42.67365$

Repeatability Standard Deviation $=14.36604$

Reproducibility Standard Deviation $=44.25641$

Zn

Initial Preparation of Test Result Data for Material: 2 (Cereal B)

\begin{tabular}{cccccc}
\hline $\begin{array}{c}\text { Laboratory } \\
\text { Number }\end{array}$ & $\begin{array}{c}\text { Cell } \\
\text { Mean }\end{array}$ & Cell & $\boldsymbol{d}$ & $\boldsymbol{h}$ & $\boldsymbol{k}$ \\
\hline 1 & 694.40 & 4.83 & 25.25 & 0.47 & 0.28 \\
2 & 716.23 & 5.56 & 47.09 & 0.87 & 0.32 \\
9 & 610.00 & 10.00 & -59.14 & -1.10 & 0.57 \\
10 & 587.40 & 37.51 & -81.75 & -1.51 & 2.15 \\
11 & 675.00 & 17.35 & 5.86 & 0.11 & 0.99 \\
13 & 573.33 & 5.77 & -95.81 & -1.78 & 0.33 \\
15 & 723.33 & 20.98 & 54.19 & 1.00 & 1.20 \\
20 & 730.00 & 5.00 & 60.86 & 1.13 & 0.29 \\
22 & 687.67 & 6.11 & 18.52 & 0.34 & 0.35 \\
26 & 679.33 & 4.04 & 10.19 & 0.19 & 0.23 \\
29 & 686.00 & 7.00 & 16.86 & 0.31 & 0.40 \\
30 & 674.00 & 3.00 & 4.86 & 0.09 & 0.17 \\
31 & 793.33 & 40.41 & 124.19 & 2.30 & 2.31 \\
33 & 650.00 & 36.06 & -19.14 & -0.35 & 2.06 \\
34 & 622.33 & 15.95 & -46.81 & -0.87 & 0.91 \\
37 & 660.33 & 8.08 & -8.81 & -0.16 & 0.46 \\
39 & 619.53 & 17.36 & -49.61 & -0.92 & 0.99 \\
40 & 684.33 & 7.02 & 15.19 & 0.28 & 0.40 \\
41 & 706.67 & 5.77 & 37.52 & 0.70 & 0.33 \\
\hline
\end{tabular}

Average of cell averages $=669.14500$

Standard Deviation of cell averages $=53.96865$

Repeatability Standard Deviation $=17.47966$

Reproducibility Standard Deviation $=55.82389$

Zn

Initial Preparation of Test Result Data for Material: 3 (Cereal C)

\begin{tabular}{cccccc}
\hline $\begin{array}{c}\text { Laboratory } \\
\text { Number }\end{array}$ & $\begin{array}{c}\text { Cell } \\
\text { Mean }\end{array}$ & Cell & $\boldsymbol{d}$ & $\boldsymbol{h}$ & \multicolumn{1}{c}{ SD } \\
\hline 1 & 695.57 & 6.12 & 16.85 & 0.26 & 0.25 \\
2 & 715.63 & 5.18 & 36.92 & 0.56 & 0.21 \\
9 & 820.00 & 20.00 & 141.28 & 2.15 & 0.82 \\
10 & 521.63 & 65.97 & -157.08 & -2.39 & 2.71 \\
11 & 688.33 & 22.23 & 9.62 & 0.15 & 0.91 \\
13 & 586.67 & 5.77 & -92.05 & -1.40 & 0.24 \\
15 & 756.00 & 13.11 & 77.28 & 1.17 & 0.54 \\
20 & 743.33 & 10.41 & 64.62 & 0.98 & 0.43 \\
22 & 699.33 & 10.50 & 20.62 & 0.31 & 0.43
\end{tabular}




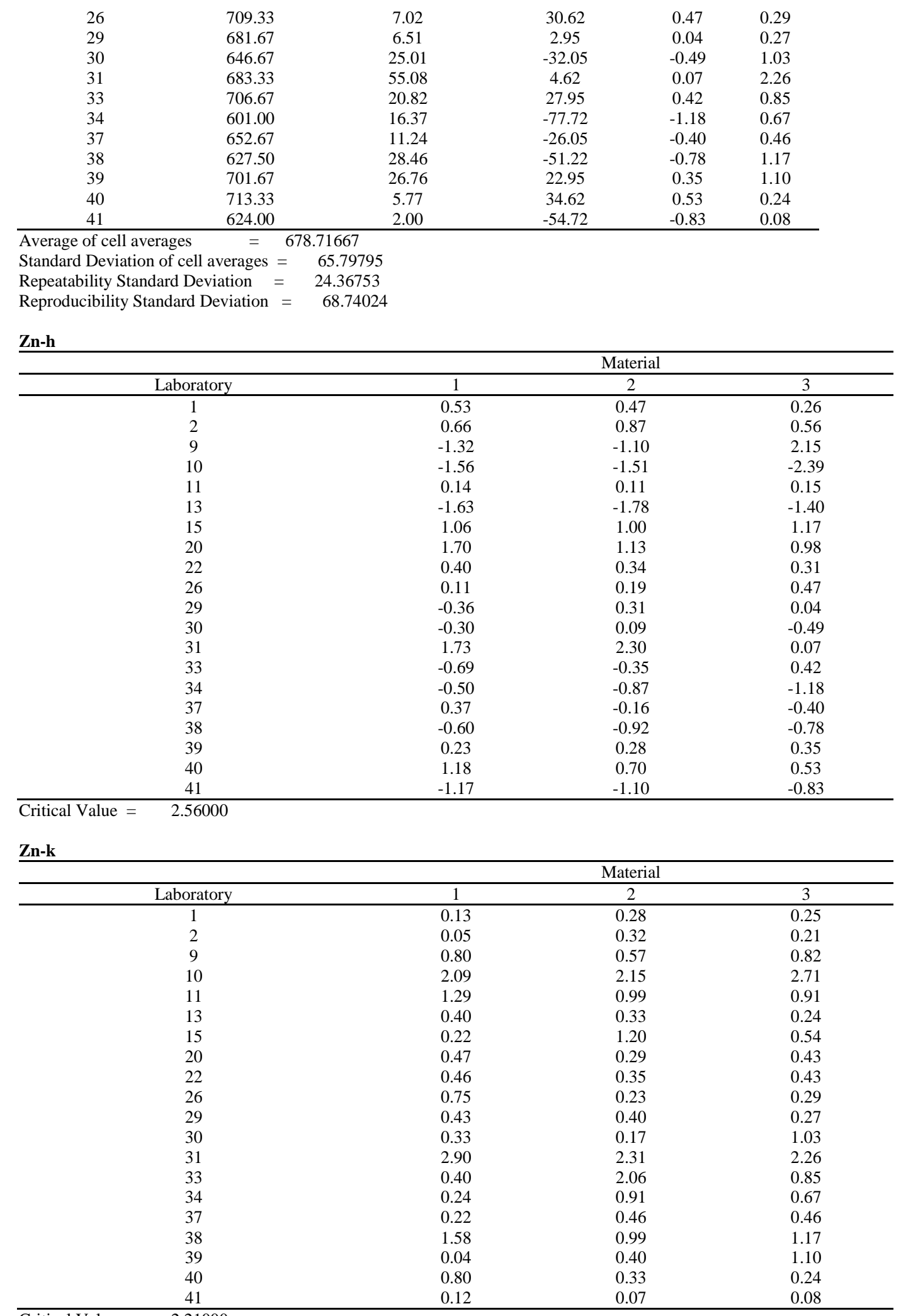

Critical Value $=\quad 2.21000$

\section{Zn-Precision Statistics}

\begin{tabular}{|c|c|c|c|c|c|c|}
\hline Material & Xbar & $S_{X}$ & $S_{r}$ & $S_{R}$ & $r$ & $\boldsymbol{R}$ \\
\hline 1 & 602.89 & 42.67 & 14.37 & 44.26 & 40.22 & 123.92 \\
\hline 2 & 669.14 & 53.97 & 17.48 & 55.82 & 48.94 & 156.31 \\
\hline 3 & 678.72 & 65.80 & 24.37 & 68.74 & 68.23 & 192.47 \\
\hline
\end{tabular}



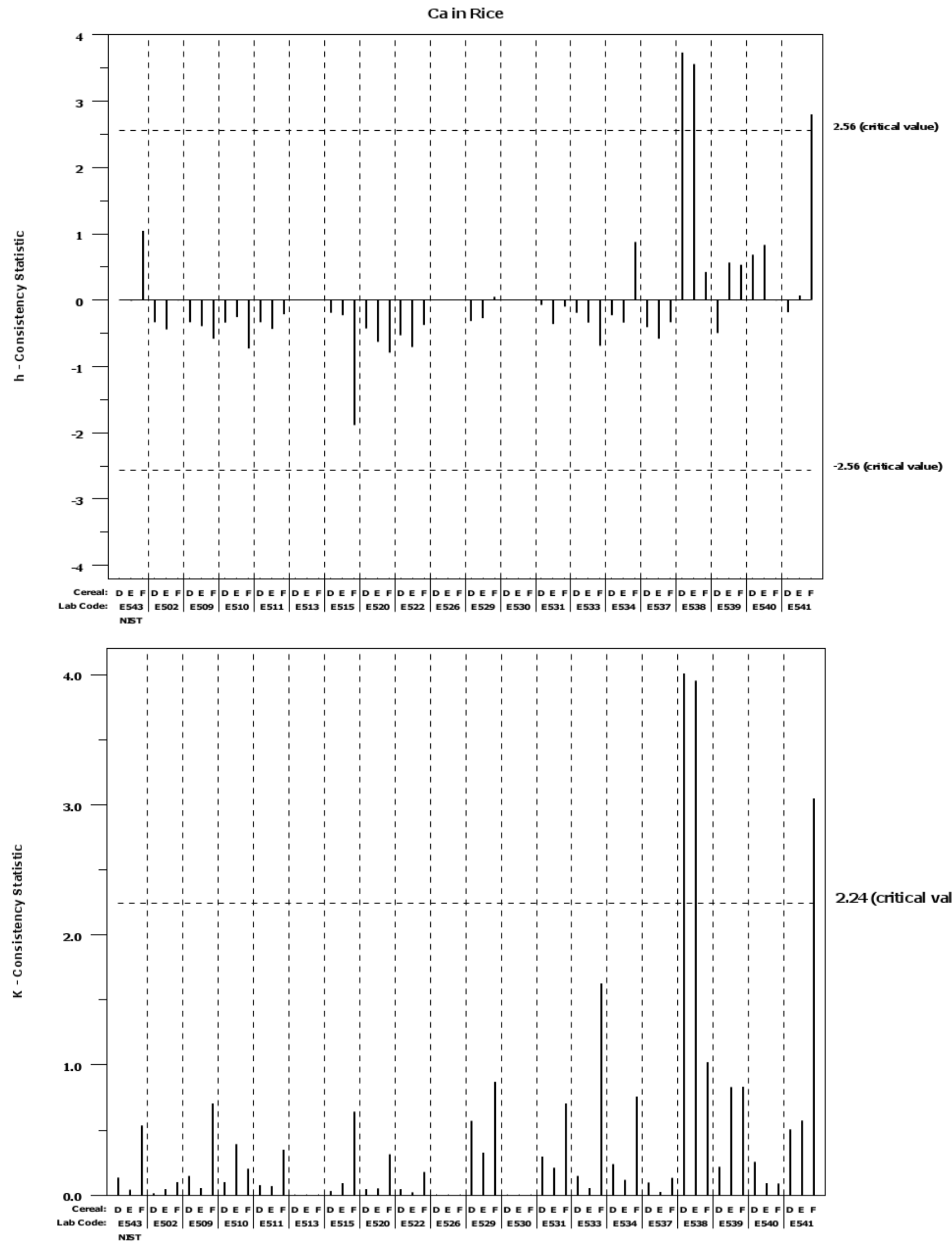

2.24 (critical value) 
Ca

Initial Preparation of Test Result Data for Material: 4 (Cereal D)

\begin{tabular}{cccccc}
\hline $\begin{array}{c}\text { Laboratory } \\
\text { Number }\end{array}$ & $\begin{array}{c}\text { Cell } \\
\text { Mean }\end{array}$ & $\begin{array}{c}\text { Cell } \\
\text { SD }\end{array}$ & $\boldsymbol{d}$ & $\boldsymbol{h}$ & $\boldsymbol{k}$ \\
\hline 1 & 282.33 & 10.50 & -0.69 & 0.00 & 0.13 \\
2 & 196.00 & 1.00 & -87.02 & -0.34 & 0.01 \\
9 & 196.67 & 11.55 & -86.35 & -0.33 & 0.15 \\
10 & 195.33 & 7.64 & -87.69 & -0.34 & 0.10 \\
11 & 196.00 & 6.00 & -87.02 & -0.34 & 0.08 \\
15 & 233.33 & 2.31 & -49.69 & -0.19 & 0.03 \\
20 & 171.33 & 3.51 & -111.69 & -0.43 & 0.04 \\
22 & 144.67 & 3.51 & -138.35 & -0.53 & 0.04 \\
29 & 201.33 & 44.60 & -81.69 & -0.32 & 0.57 \\
31 & 263.33 & 23.09 & -19.69 & -0.08 & 0.29 \\
33 & 233.33 & 11.55 & -49.69 & -0.19 & 0.15 \\
34 & 224.00 & 18.68 & -59.02 & -0.23 & 0.24 \\
37 & 176.33 & 7.51 & -106.69 & -0.41 & 0.10 \\
38 & 1248.00 & 314.45 & 964.98 & 3.73 & 4.01 \\
39 & 154.33 & 17.04 & -128.69 & -0.50 & 0.22 \\
40 & 460.00 & 20.00 & 176.98 & 0.68 & 0.26 \\
41 & 235.00 & 39.59 & -48.02 & -0.19 & 0.50 \\
\hline
\end{tabular}

Average of cell averages $\quad=\quad 283.01961$

Standard Deviation of cell averages $=258.61948$

Repeatability Standard Deviation $=78.42894$

Reproducibility Standard Deviation $=266.42966$

Ca

Initial Preparation of Test Result Data for Material: 5 (Cereal E)

\begin{tabular}{cccccc}
\hline $\begin{array}{c}\text { Laboratory } \\
\text { Number }\end{array}$ & $\begin{array}{c}\text { Cell } \\
\text { Mean }\end{array}$ & Cell & $\boldsymbol{d}$ & $\boldsymbol{h}$ & $\boldsymbol{k}$ \\
\hline 1 & 279.67 & 4.51 & -2.25 & -0.01 & 0.04 \\
2 & 196.67 & 5.03 & -85.25 & -0.45 & 0.05 \\
9 & 206.67 & 5.77 & -75.25 & -0.39 & 0.05 \\
10 & 232.67 & 43.02 & -49.25 & -0.26 & 0.39 \\
11 & 198.67 & 7.57 & -83.25 & -0.44 & 0.07 \\
15 & 238.33 & 9.87 & -43.59 & -0.23 & 0.09 \\
20 & 161.33 & 5.51 & -120.59 & -0.63 & 0.05 \\
22 & 146.33 & 2.08 & -135.59 & -0.71 & 0.02 \\
29 & 229.67 & 35.92 & -52.25 & -0.27 & 0.33 \\
31 & 213.33 & 23.09 & -68.59 & -0.36 & 0.21 \\
33 & 216.67 & 5.77 & -65.25 & -0.34 & 0.05 \\
34 & 216.67 & 12.66 & -65.25 & -0.34 & 0.12 \\
37 & 170.67 & 2.52 & -111.25 & -0.58 & 0.02 \\
38 & 960.67 & 435.18 & 678.75 & 3.56 & 3.95 \\
49 & 390.00 & 91.33 & 108.08 & 0.57 & 0.83 \\
41 & 440.00 & 10.00 & 158.08 & 0.83 & 0.09 \\
\hline
\end{tabular}

Average of cell averages $\quad=\quad 281.92157$

Standard Deviation of cell averages $=190.88215$

Repeatability Standard Deviation $=110.06041$

Reproducibility Standard Deviation $=210.97754$

Ca

Initial Preparation of Test Result Data for Material: 6 (Cereal F)

\begin{tabular}{cccccc}
\hline $\begin{array}{c}\text { Laboratory } \\
\text { Number }\end{array}$ & $\begin{array}{c}\text { Cell } \\
\text { Mean }\end{array}$ & $\begin{array}{c}\text { Cell } \\
\text { SD }\end{array}$ & $\boldsymbol{d}$ & $\boldsymbol{h}$ & $\boldsymbol{k}$ \\
\hline 1 & 21131.00 & 920.23 & 2847.37 & 1.04 & 0.53 \\
2 & 18259.33 & 170.39 & -24.29 & -0.01 & 0.10 \\
9 & 16700.00 & 1212.44 & -1583.63 & -0.58 & 0.70 \\
10 & 16296.67 & 346.46 & -1986.96 & -0.73 & 0.20 \\
11 & 17700.00 & 600.00 & -583.63 & -0.21 & 0.35 \\
15 & 13128.33 & 1103.07 & -5155.29 & -1.89 & 0.64 \\
20 & 16120.33 & 538.26 & -2163.29 & -0.79 & 0.31 \\
22 & 17254.67 & 303.90 & -1028.96 & -0.38 & 0.18 \\
29 & 18416.67 & 1500.28 & 133.04 & 0.05 & 0.87 \\
31 & 18013.33 & 1213.44 & -270.29 & -0.10 & 0.70 \\
33 & 16400.00 & 2805.35 & -1883.63 & -0.69 & 1.63 \\
34 & 20666.67 & 1305.12 & 2383.04 & 0.87 & 0.76 \\
37 & 17364.00 & 227.53 & -919.63 & -0.34 & 0.13
\end{tabular}




\begin{tabular}{cccccc}
38 & 19438.67 & 1759.52 & 1155.04 & 0.42 & 1.02 \\
39 & 19733.33 & 1436.43 & 1449.71 & 0.53 & 0.83 \\
40 & 18266.67 & 152.75 & -16.96 & -0.01 & 0.09 \\
41 & 25932.00 & 5254.32 & 7648.37 & 2.80 & 3.05 \\
\hline
\end{tabular}

Average of cell averages $\quad=18283.62745$

Standard Deviation of cell averages $=2730.81197$

Repeatability Standard Deviation $=1724.62449$

Reproducibility Standard Deviation $=3072.49417$

Ca-h

\begin{tabular}{cccc}
\hline & \multicolumn{3}{c}{ Material } \\
\hline Laboratory & 1 & 2 & 3 \\
\hline 1 & 0.00 & -0.01 & 1.04 \\
2 & -0.34 & -0.45 & -0.01 \\
9 & -0.33 & -0.39 & -0.58 \\
10 & -0.34 & -0.26 & -0.73 \\
11 & -0.34 & -0.44 & -0.21 \\
15 & -0.19 & -0.23 & -1.89 \\
20 & -0.43 & -0.63 & -0.79 \\
22 & -0.53 & -0.71 & -0.38 \\
29 & -0.32 & -0.27 & 0.05 \\
31 & -0.08 & -0.36 & -0.10 \\
33 & -0.19 & -0.34 & -0.69 \\
34 & -0.23 & -0.34 & 0.87 \\
37 & -0.41 & -0.58 & -0.34 \\
38 & 3.73 & 3.56 & 0.42 \\
39 & -0.50 & 0.57 & 0.53 \\
40 & 0.68 & 0.83 & -0.01 \\
41 & -0.19 & 0.07 & 2.80 \\
\hline
\end{tabular}

Critical Value $=2.51000$

Ca-k

\begin{tabular}{cccc}
\hline Ca-k & \multicolumn{3}{l}{ Material } \\
\hline Laboratory & 1 & 2 & 3 \\
\hline 1 & 0.13 & 0.04 & 0.53 \\
2 & 0.01 & 0.05 & 0.10 \\
9 & 0.15 & 0.05 & 0.70 \\
10 & 0.10 & 0.39 & 0.20 \\
11 & 0.08 & 0.07 & 0.35 \\
15 & 0.03 & 0.09 & 0.64 \\
20 & 0.04 & 0.05 & 0.31 \\
22 & 0.04 & 0.02 & 0.18 \\
29 & 0.57 & 0.33 & 0.87 \\
31 & 0.29 & 0.21 & 0.70 \\
33 & 0.15 & 0.05 & 1.63 \\
34 & 0.24 & 0.12 & 0.76 \\
37 & 0.10 & 0.02 & 0.13 \\
38 & 4.01 & 3.95 & 1.02 \\
39 & 0.22 & 0.83 & 0.83 \\
40 & 0.26 & 0.09 & 0.09 \\
41 & 0.50 & 0.57 & 3.05 \\
\hline
\end{tabular}

Critical Value $=\quad 2.19000$

Ca-Precision Statistics

\begin{tabular}{ccccccc}
\hline Material & Xbar & $\boldsymbol{S}_{\boldsymbol{x}}$ & $\boldsymbol{S}_{\boldsymbol{r}}$ & $\boldsymbol{S}_{\boldsymbol{R}}$ & $\boldsymbol{r}$ \\
\hline 4 & 283.02 & 258.62 & 78.43 & 266.43 & 219.60 & 746.00 \\
5 & 281.92 & 190.88 & 110.06 & 210.98 & 308.17 & 590.74 \\
6 & 18283.63 & 2730.81 & 1724.62 & 3072.49 & 4828.95 & 8602.98 \\
\hline
\end{tabular}


Fe in Rice
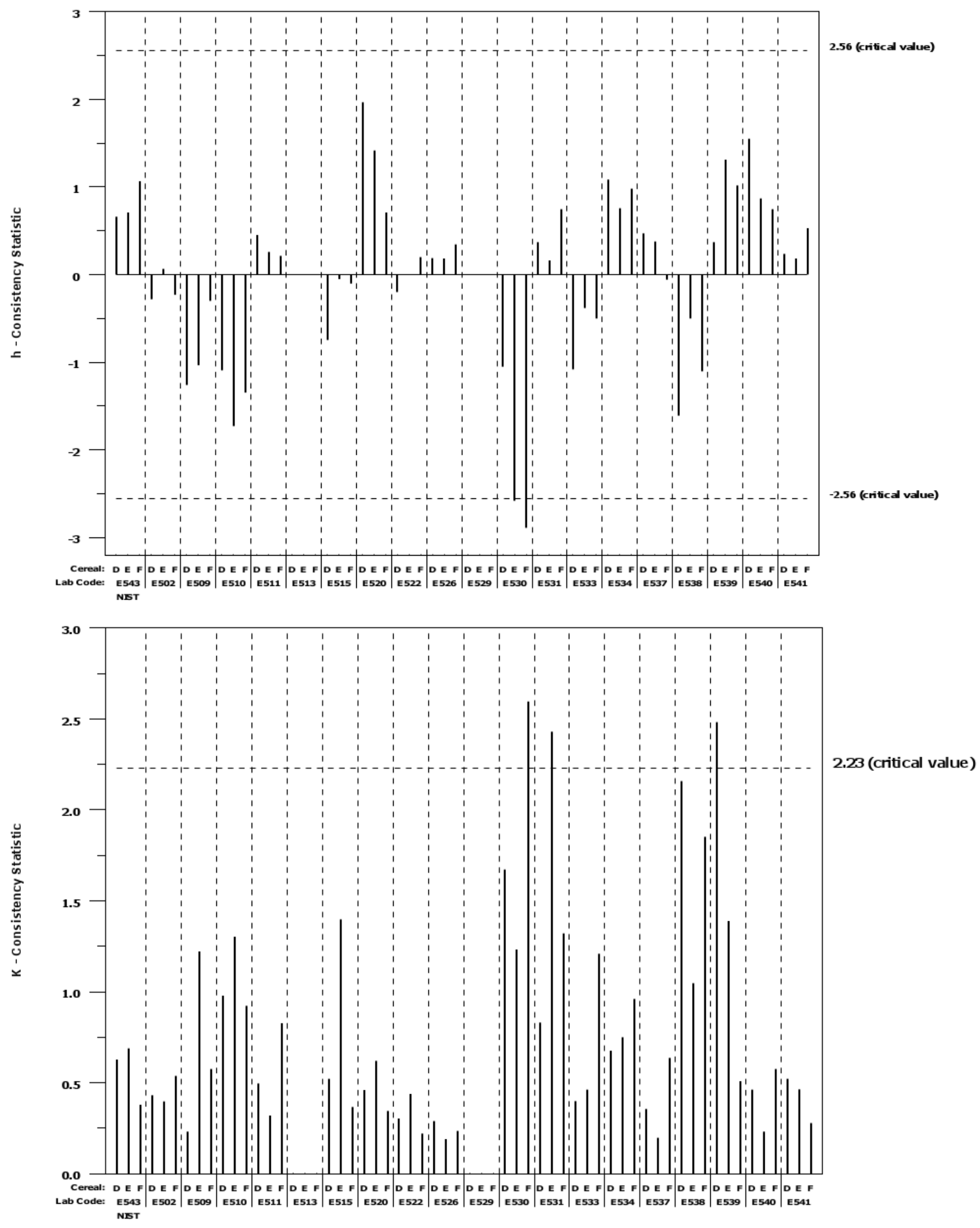
$\mathrm{Fe}$

Initial Preparation of Test Result Data for Material: 4 (Cereal D)

\begin{tabular}{cccccc}
\hline $\begin{array}{c}\text { Laboratory } \\
\text { Number }\end{array}$ & $\begin{array}{c}\text { Cell } \\
\text { Mean }\end{array}$ & Cell & $\boldsymbol{d}$ & $\boldsymbol{h}$ & $\boldsymbol{k}$ \\
\hline 1 & 524.00 & 15.72 & 24.24 & 0.66 & 0.63 \\
2 & 489.40 & 10.78 & -10.36 & -0.28 & 0.43 \\
9 & 453.33 & 5.77 & -46.43 & -1.26 & 0.23 \\
10 & 459.50 & 24.55 & -40.26 & -1.09 & 0.98 \\
11 & 516.33 & 12.42 & 16.57 & 0.45 & 0.50 \\
15 & 472.33 & 13.05 & -27.43 & -0.75 & 0.52 \\
20 & 572.00 & 11.53 & 72.24 & 1.96 & 0.46 \\
22 & 492.33 & 7.57 & -7.43 & -0.20 & 0.30 \\
26 & 506.67 & 7.23 & 6.91 & 0.19 & 0.29 \\
30 & 461.00 & 41.90 & -38.76 & -1.05 & 1.67 \\
31 & 513.33 & 20.82 & 13.57 & 0.37 & 0.83 \\
33 & 460.00 & 10.00 & -39.76 & -1.08 & 0.40 \\
34 & 539.67 & 16.92 & 39.91 & 1.09 & 0.67 \\
37 & 517.00 & 8.89 & 17.24 & 0.47 & 0.35 \\
38 & 440.47 & 54.12 & -59.29 & -1.61 & 2.16 \\
49 & 513.33 & 62.27 & 13.57 & 0.37 & 2.48 \\
41 & 556.67 & 11.55 & 56.91 & 1.55 & 0.46 \\
\hline
\end{tabular}

Average of cell averages $\quad=\quad 499.76111$

Standard Deviation of cell averages $=36.77321$

Repeatability Standard Deviation $=25.08917$

Reproducibility Standard Deviation $=42.09410$

Fe

Initial Preparation of Test Result Data for Material: 5 (Cereal E)

\begin{tabular}{cccccc}
\hline $\begin{array}{c}\text { Laboratory } \\
\text { Number }\end{array}$ & $\begin{array}{c}\text { Cell } \\
\text { Mean }\end{array}$ & Cell & $\boldsymbol{d}$ & $\boldsymbol{h}$ & $\boldsymbol{k}$ \\
\hline 1 & 533.67 & 17.21 & 43.49 & 0.71 & 0.69 \\
2 & 494.17 & 9.92 & 3.99 & 0.06 & 0.40 \\
9 & 426.67 & 30.55 & -63.51 & -1.04 & 1.22 \\
10 & 384.07 & 32.58 & -106.11 & -1.73 & 1.30 \\
11 & 506.00 & 8.00 & 15.82 & 0.26 & 0.32 \\
15 & 487.00 & 35.00 & -3.18 & -0.05 & 1.40 \\
20 & 577.00 & 15.52 & 86.82 & 1.42 & 0.62 \\
22 & 490.33 & 10.97 & 0.15 & 0.00 & 0.44 \\
26 & 501.33 & 4.73 & 11.15 & 0.18 & 0.19 \\
30 & 331.67 & 30.83 & -158.51 & -2.58 & 1.23 \\
31 & 500.00 & 60.83 & 9.82 & 0.16 & 2.43 \\
33 & 466.67 & 11.55 & -23.51 & -0.38 & 0.46 \\
34 & 536.67 & 18.77 & 46.49 & 0.76 & 0.75 \\
37 & 513.33 & 4.93 & 23.15 & 0.38 & 0.20 \\
39 & 459.37 & 26.21 & -30.81 & -0.50 & 1.05 \\
40 & 570.67 & 34.78 & 80.49 & 1.31 & 1.39 \\
41 & 543.33 & 5.77 & 53.15 & 0.87 & 0.23 \\
\hline
\end{tabular}

Average of cell averages $=490.18148$

Standard Deviation of cell averages $=61.34454$

Repeatability Standard Deviation $=25.04024$

Reproducibility Standard Deviation $=64.66190$

Fe

Initial Preparation of Test Result Data for Material: 6 (Cereal F)

\begin{tabular}{cccccc}
\hline $\begin{array}{c}\text { Laboratory } \\
\text { Number }\end{array}$ & $\begin{array}{c}\text { Cell } \\
\text { Mean }\end{array}$ & Cell & $\boldsymbol{d}$ & $\boldsymbol{h}$ & $\boldsymbol{k}$ \\
\hline 1 & 738.33 & SD & 10.07 & 105.31 & 1.06 \\
2 & 610.33 & 14.26 & -22.69 & -0.23 & 0.38 \\
9 & 603.33 & 15.28 & -29.69 & -0.30 & 0.57 \\
10 & 499.77 & 24.54 & -133.26 & -1.35 & 0.92 \\
11 & 654.00 & 22.00 & 20.98 & 0.21 & 0.83 \\
15 & 622.67 & 9.71 & -10.36 & -0.10 & 0.37 \\
20 & 703.00 & 9.17 & 69.98 & 0.71 & 0.34 \\
22 & 652.67 & 5.86 & 19.64 & 0.20 & 0.22 \\
26 & 667.00 & 6.24 & 33.98 & 0.34 & 0.23 \\
30 & 346.67 & 69.04 & -286.36 & -2.89 & 2.59 \\
31 & 706.67 & 35.12 & 73.64 & 0.74 & 1.32 \\
33 & 583.33 & 32.15 & -49.69 & -0.50 & 1.21 \\
34 & 730.00 & 25.51 & 96.98 & 0.98
\end{tabular}




\begin{tabular}{llllll}
37 & 627.33 & 16.92 & -5.69 & -0.06 & 0.64 \\
38 & 523.67 & 49.25 & -109.36 & -1.10 & 1.85 \\
39 & 733.67 & 13.50 & 100.64 & 1.02 & 0.51 \\
40 & 706.67 & 15.28 & 73.64 & 0.74 & 0.57 \\
41 & 685.33 & 7.37 & 52.31 & 0.53 & 0.28 \\
\hline
\end{tabular}

Average of cell averages $\quad=\quad 633.02407$

Standard Deviation of cell averages $=\quad 99.05590$

Repeatability Standard Deviation $=26.60641$

Reproducibility Standard Deviation $=101.41009$

Fe-h

\begin{tabular}{lccc}
\hline & \multicolumn{3}{c}{ Material } \\
\hline Laboratory & 1 & 2 & 3 \\
\hline 1 & 0.66 & 0.71 & 1.06 \\
2 & -0.28 & 0.06 & -0.23 \\
9 & -1.26 & -1.04 & -0.30 \\
10 & -1.09 & -1.73 & -1.35 \\
11 & 0.45 & 0.26 & 0.21 \\
15 & -0.75 & -0.05 & -0.10 \\
20 & 1.96 & 1.42 & 0.71 \\
22 & -0.20 & 0.00 & 0.20 \\
26 & 0.19 & 0.18 & 0.34 \\
30 & -1.05 & -2.58 & -2.89 \\
31 & 0.37 & 0.16 & 0.74 \\
33 & -1.08 & -0.38 & -0.50 \\
34 & 1.09 & 0.76 & 0.98 \\
37 & 0.47 & 0.38 & -0.06 \\
38 & -1.61 & -0.50 & -1.10 \\
39 & 0.37 & 1.31 & 1.02 \\
40 & 1.55 & 0.87 & 0.74 \\
41 & 0.23 & 0.18 & 0.53 \\
\hline
\end{tabular}

Critical Value $=2.53000$

Fe-k

\begin{tabular}{cccc}
\hline & \multicolumn{3}{c}{ Material } \\
\hline Laboratory & 1 & 2 & 3 \\
\hline 1 & 0.63 & 0.69 & 0.38 \\
2 & 0.43 & 0.40 & 0.54 \\
9 & 0.23 & 1.22 & 0.57 \\
10 & 0.98 & 1.30 & 0.92 \\
11 & 0.50 & 0.32 & 0.83 \\
15 & 0.52 & 1.40 & 0.37 \\
20 & 0.46 & 0.62 & 0.34 \\
22 & 0.30 & 0.44 & 0.22 \\
26 & 0.29 & 0.19 & 0.23 \\
30 & 1.67 & 1.23 & 2.59 \\
31 & 0.83 & 2.43 & 1.32 \\
33 & 0.40 & 0.46 & 1.21 \\
34 & 0.67 & 0.75 & 0.96 \\
37 & 0.35 & 0.20 & 0.64 \\
38 & 2.16 & 1.05 & 1.85 \\
39 & 2.48 & 1.39 & 0.51 \\
40 & 0.46 & 0.23 & 0.57 \\
41 & 0.52 & 0.46 & 0.28 \\
\hline
\end{tabular}

Critical Value $=2.20000$

Fe-Precision Statistics

\begin{tabular}{ccccccc}
\hline Material & Xbar & $\boldsymbol{s}_{\boldsymbol{x}}$ & $\boldsymbol{S}_{\boldsymbol{r}}$ & $\boldsymbol{S}_{\boldsymbol{R}}$ & $\boldsymbol{r}$ \\
\hline 4 & 499.76 & 36.77 & 25.09 & 42.09 & 70.25 & 117.86 \\
5 & 490.18 & 61.34 & 25.04 & 64.66 & 70.11 & 181.05 \\
6 & 633.02 & 99.06 & 26.61 & 101.41 & 74.50 & 283.95 \\
\hline
\end{tabular}


$\mathrm{Zn}$ in Rice
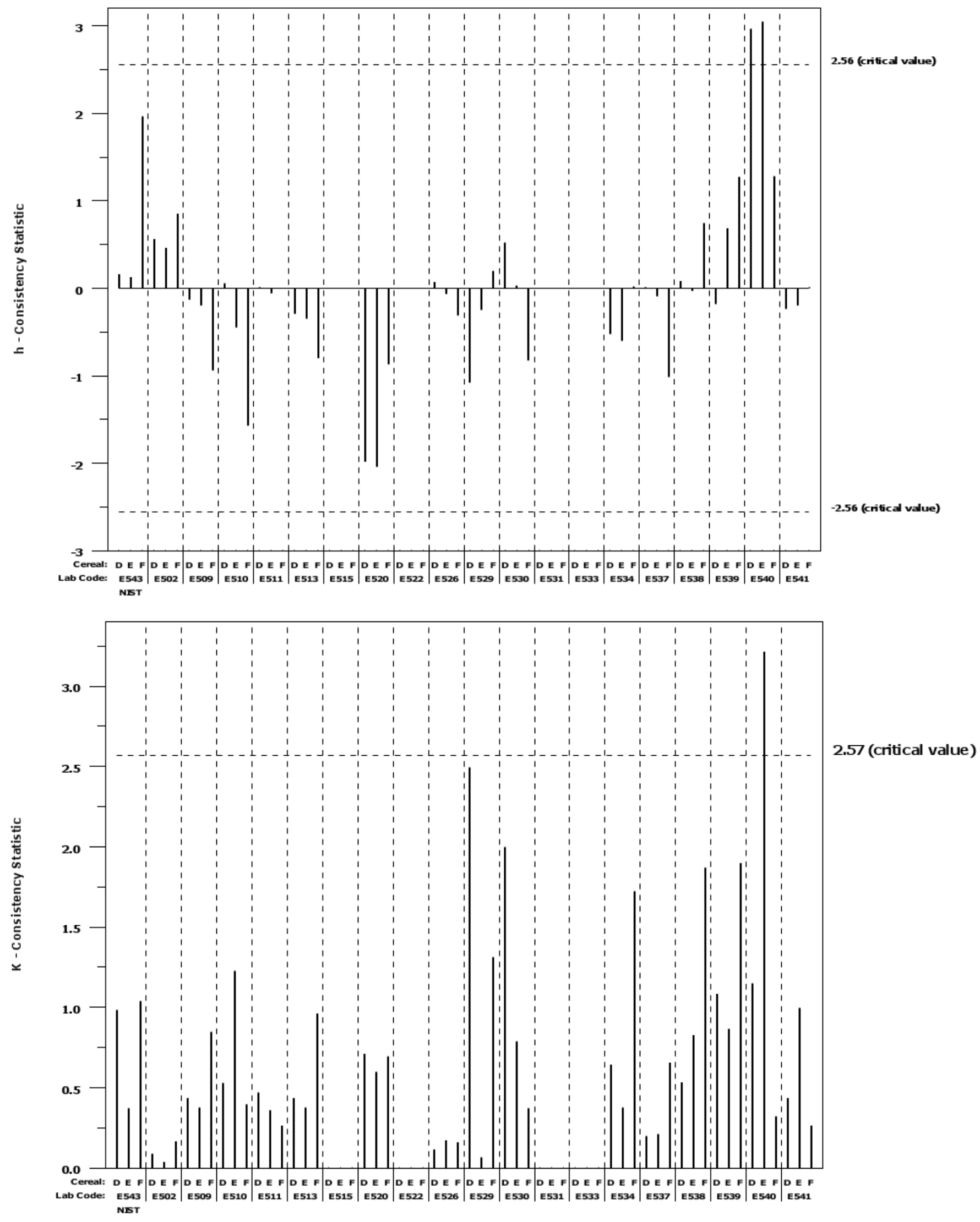
Zn

Initial Preparation of Test Result Data for Material: 4 (Cereal D)

\begin{tabular}{cccccc}
\hline $\begin{array}{c}\text { Laboratory } \\
\text { Number }\end{array}$ & $\begin{array}{c}\text { Cell } \\
\text { Mean }\end{array}$ & Cell & d & $\boldsymbol{h}$ & $\boldsymbol{k}$ \\
\hline 1 & 21.10 & 1.31 & 0.97 & 0.16 & 0.98 \\
2 & 23.57 & 0.12 & 3.44 & 0.56 & 0.09 \\
9 & 19.33 & 0.58 & -0.80 & -0.13 & 0.43 \\
10 & 20.47 & 0.70 & 0.34 & 0.05 & 0.53 \\
11 & 20.20 & 0.62 & 0.07 & 0.01 & 0.47 \\
13 & 18.33 & 0.58 & -1.80 & -0.29 & 0.43 \\
20 & 7.97 & 0.95 & -12.16 & -1.98 & 0.71 \\
26 & 20.57 & 0.15 & 0.44 & 0.07 & 0.11 \\
29 & 13.50 & 3.32 & -6.63 & -1.08 & 2.49 \\
30 & 23.33 & 2.66 & 3.20 & 0.52 & 2.00 \\
34 & 16.90 & 0.85 & -3.23 & -0.53 & 0.64 \\
37 & 20.20 & 0.26 & 0.07 & 0.01 & 0.20 \\
38 & 20.63 & 0.71 & 0.50 & 0.08 & 0.53 \\
39 & 19.00 & 1.44 & -1.13 & -0.18 & 1.08 \\
40 & 38.33 & 1.53 & 18.20 & 2.97 \\
41 & 18.67 & 0.58 & -1.46 & -0.24 & 0.43 \\
\hline Average of cell averages & $=$ & & &
\end{tabular}

Zn

Initial Preparation of Test Result Data for Material: 5 (Cereal E)

\begin{tabular}{|c|c|c|c|c|c|}
\hline $\begin{array}{c}\text { Laboratory } \\
\text { Number }\end{array}$ & $\begin{array}{c}\text { Cell } \\
\text { Mean }\end{array}$ & $\begin{array}{c}\text { Cell } \\
\text { SD }\end{array}$ & $d$ & $\boldsymbol{h}$ & $k$ \\
\hline 1 & 21.47 & 0.57 & 0.83 & 0.13 & 0.37 \\
\hline 2 & 23.67 & 0.06 & 3.03 & 0.46 & 0.04 \\
\hline 9 & 19.33 & 0.58 & -1.30 & -0.20 & 0.38 \\
\hline 10 & 17.67 & 1.88 & -2.97 & -0.45 & 1.23 \\
\hline 11 & 20.23 & 0.55 & -0.40 & -0.06 & 0.36 \\
\hline 13 & 18.33 & 0.58 & -2.30 & -0.35 & 0.38 \\
\hline 20 & 7.20 & 0.92 & -13.44 & -2.04 & 0.60 \\
\hline 26 & 20.20 & 0.26 & -0.44 & -0.07 & 0.17 \\
\hline 29 & 19.00 & 0.10 & -1.64 & -0.25 & 0.07 \\
\hline 30 & 20.83 & 1.21 & 0.20 & 0.03 & 0.79 \\
\hline 34 & 16.67 & 0.58 & -3.97 & -0.60 & 0.38 \\
\hline 37 & 20.03 & 0.32 & -0.60 & -0.09 & 0.21 \\
\hline 38 & 20.43 & 1.27 & -0.20 & -0.03 & 0.83 \\
\hline 39 & 25.13 & 1.33 & 4.50 & 0.68 & 0.87 \\
\hline 40 & 40.67 & 4.93 & 20.03 & 3.05 & 3.21 \\
\hline 41 & 19.33 & 1.53 & -1.30 & -0.20 & 1.00 \\
\hline Average of cell av & $=2$ & & & & \\
\hline Standard Deviatio & jerages = & & & & \\
\hline Repeatability Stan & ation = & & & & \\
\hline Reproducibility S & viation $=$ & & & & \\
\hline \\
\hline \multicolumn{6}{|c|}{ Initial Preparation of Test Result Data for Material: 6 (Cereal F) } \\
\hline Laboratory & Cell & Cell & & & \\
\hline Number & Mean & SD & $d$ & $\boldsymbol{h}$ & $k$ \\
\hline 1 & 448.33 & 18.72 & 91.17 & 1.96 & 1.04 \\
\hline 2 & 396.67 & 2.99 & 39.50 & 0.85 & 0.17 \\
\hline 9 & 313.33 & 15.28 & -43.83 & -0.94 & 0.85 \\
\hline 10 & 284.17 & 7.12 & -73.00 & -1.57 & 0.40 \\
\hline 11 & 357.33 & 4.73 & 0.17 & 0.00 & 0.26 \\
\hline 13 & 320.00 & 17.32 & -37.16 & -0.80 & 0.96 \\
\hline 20 & 316.67 & 12.50 & -40.50 & -0.87 & 0.69 \\
\hline 26 & 342.67 & 2.89 & -14.50 & -0.31 & 0.16 \\
\hline 29 & 366.33 & 23.63 & 9.17 & 0.20 & 1.31 \\
\hline 30 & 318.80 & 6.70 & -38.36 & -0.83 & 0.37 \\
\hline 34 & 358.00 & 31.05 & 0.84 & 0.02 & 1.72 \\
\hline 37 & 310.00 & 11.79 & -47.16 & -1.02 & 0.65 \\
\hline 38 & 391.67 & 33.68 & 34.50 & 0.74 & 1.87 \\
\hline 39 & 416.33 & 34.20 & 59.17 & 1.27 & 1.90 \\
\hline 40 & 416.67 & 5.77 & 59.50 & 1.28 & 0.32 \\
\hline 41 & 357.67 & 4.73 & 0.50 & 0.01 & 0.26 \\
\hline
\end{tabular}


Average of cell averages $\quad=\quad 357.16458$

Standard Deviation of cell averages $=46.45193$

Repeatability Standard Deviation $=18.02046$

Reproducibility Standard Deviation $=48.72651$

Zn-h

\begin{tabular}{cccc}
\hline & \multicolumn{3}{c}{ Material } \\
\hline Laboratory & 1 & 2 & 3 \\
\hline 1 & 0.16 & 0.13 & 1.96 \\
2 & 0.56 & 0.46 & 0.85 \\
9 & -0.13 & -0.20 & -0.94 \\
10 & 0.05 & -0.45 & -1.57 \\
11 & 0.01 & -0.06 & 0.00 \\
13 & -0.29 & -0.35 & -0.80 \\
20 & -1.98 & -2.04 & -0.87 \\
26 & 0.07 & -0.07 & -0.31 \\
29 & -1.08 & -0.25 & 0.20 \\
30 & 0.52 & 0.03 & -0.83 \\
34 & -0.53 & -0.60 & 0.02 \\
37 & 0.01 & -0.09 & -1.02 \\
38 & 0.08 & -0.03 & 0.74 \\
39 & -0.18 & 0.68 & 1.27 \\
40 & 2.97 & 3.05 & 1.28 \\
41 & -0.24 & -0.20 & 0.01 \\
\hline
\end{tabular}

Critical Value $=\quad 2.49000$

Zn-k

\begin{tabular}{cccc}
\hline & \multicolumn{3}{c}{ Material } \\
\hline Laboratory & 1 & 2 & 3 \\
\hline 1 & 0.98 & 0.37 & 1.04 \\
2 & 0.09 & 0.04 & 0.17 \\
9 & 0.43 & 0.38 & 0.85 \\
10 & 0.53 & 1.23 & 0.40 \\
11 & 0.47 & 0.36 & 0.26 \\
13 & 0.43 & 0.38 & 0.96 \\
20 & 0.71 & 0.60 & 0.69 \\
26 & 0.11 & 0.17 & 0.16 \\
29 & 2.49 & 0.07 & 1.31 \\
30 & 2.00 & 0.79 & 0.37 \\
34 & 0.64 & 0.38 & 1.72 \\
37 & 0.20 & 0.21 & 0.65 \\
38 & 0.53 & 0.83 & 1.87 \\
39 & 1.08 & 0.87 & 1.90 \\
40 & 1.15 & 3.21 & 0.32 \\
41 & 0.43 & 1.00 & 0.26 \\
\hline
\end{tabular}

Critical Value $=2.18000$

Zn-Precision Statistics

\begin{tabular}{ccccccc}
\hline Material & Xbar & $\boldsymbol{s}_{\boldsymbol{x}}$ & $\boldsymbol{s}_{\boldsymbol{r}}$ & $\boldsymbol{S}_{\boldsymbol{R}}$ & $\boldsymbol{r}$ \\
\hline 4 & 20.13 & 6.13 & 1.33 & 6.23 & 3.72 & 17.44 \\
5 & 20.64 & 6.58 & 1.54 & 6.69 & 4.30 & 18.74 \\
6 & 357.16 & 46.45 & 18.02 & 48.73 & 50.46 & 136.43 \\
\hline
\end{tabular}




\section{APPENDIX D Consensus Plots}

The following 18 plots represent the consensus results for Ca, Fe and Zn (mg/kg) in the six materials (Cereals A-F). The DerSimonian-Laird (DSL) consensus means are represented by the dark solid lines with \pm 1, 2, 3, 4, 5, 6 Horn-Horn-Duncan (HHD) standard uncertainty lines. The median value for the triplicate data are represented as circles $(\bullet)$ with the corresponding vertical lines representing the range of the values (min to max) for each individual laboratory. 


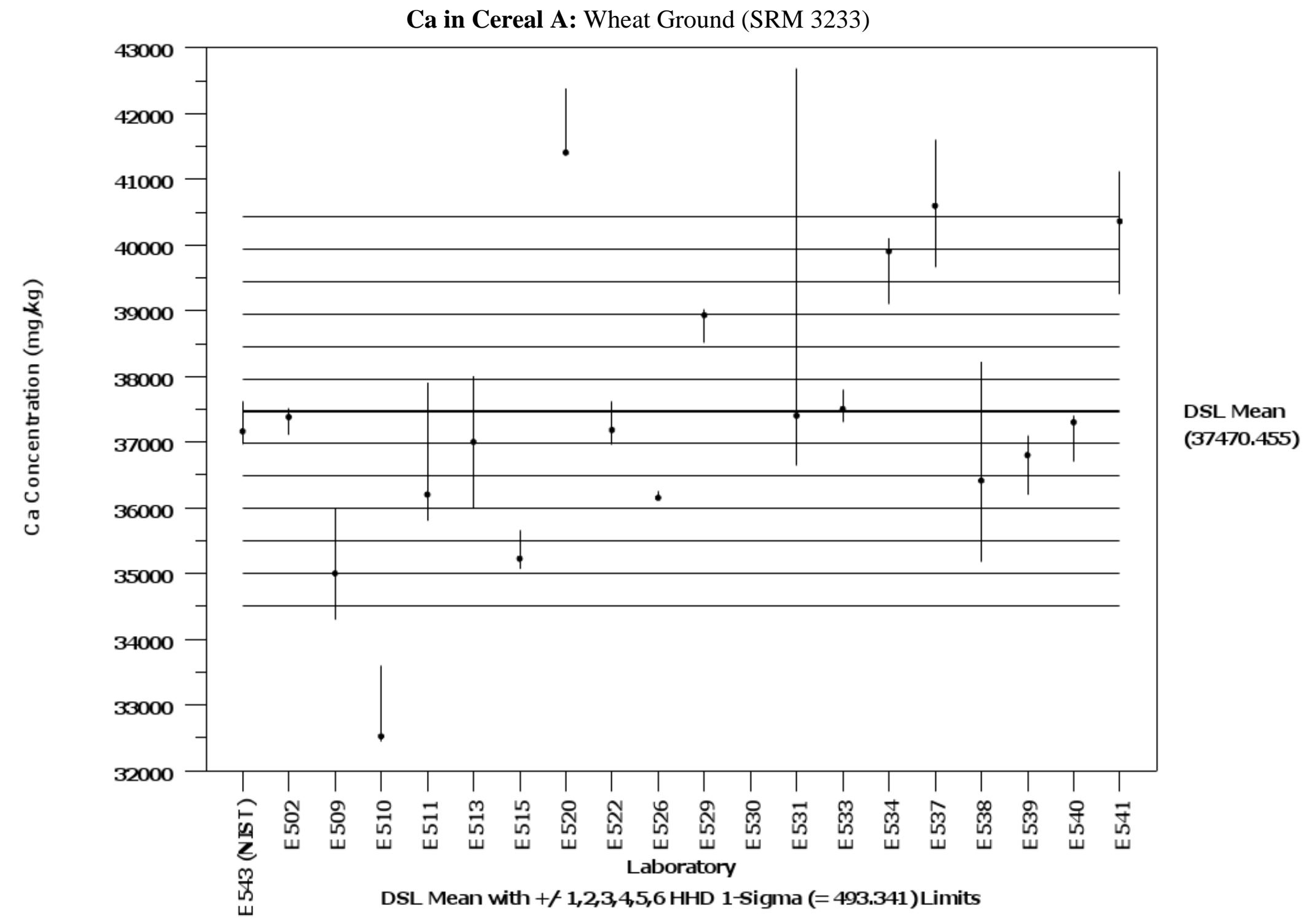




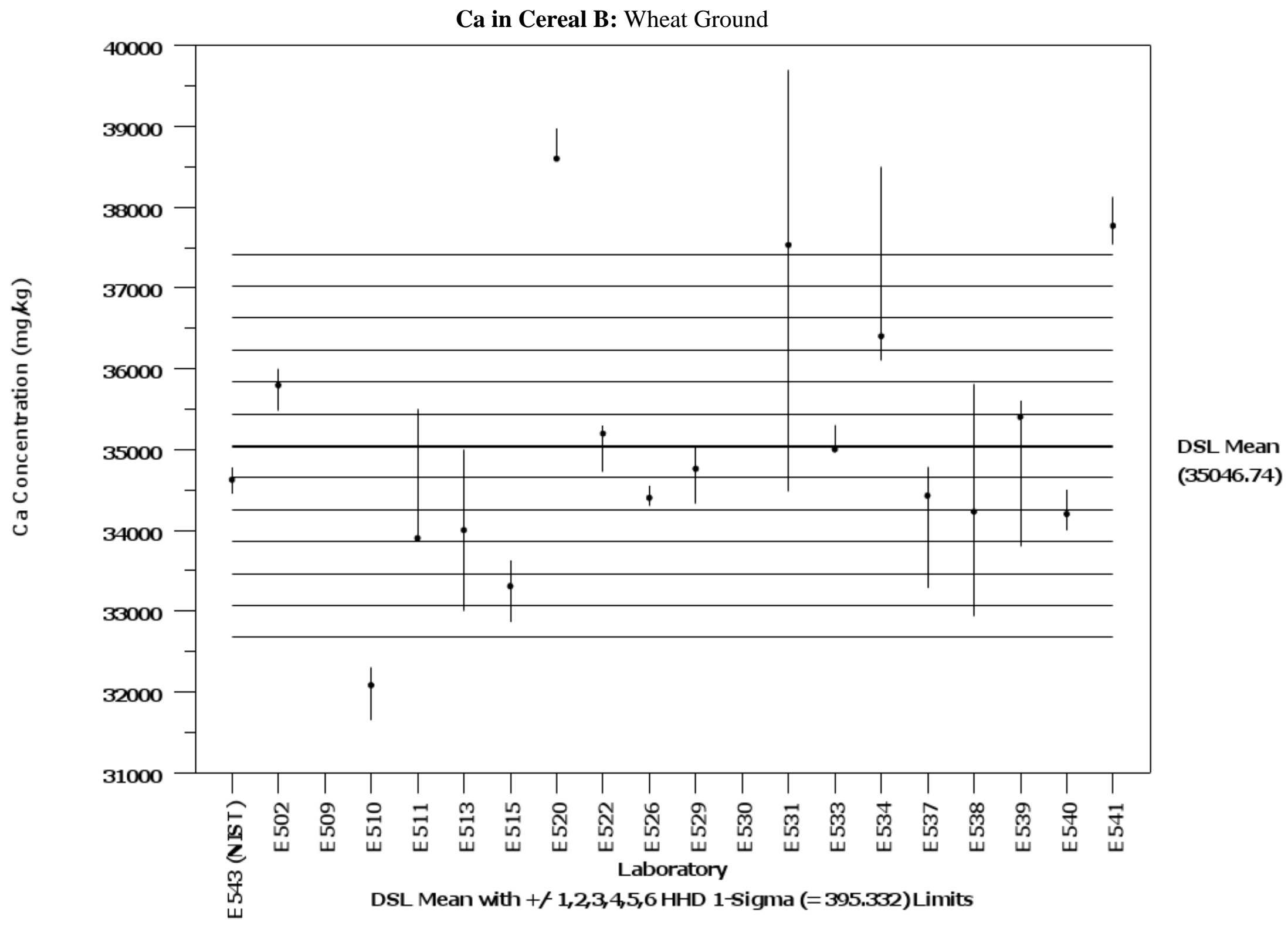


Ca in Cereal C: Wheat Flake

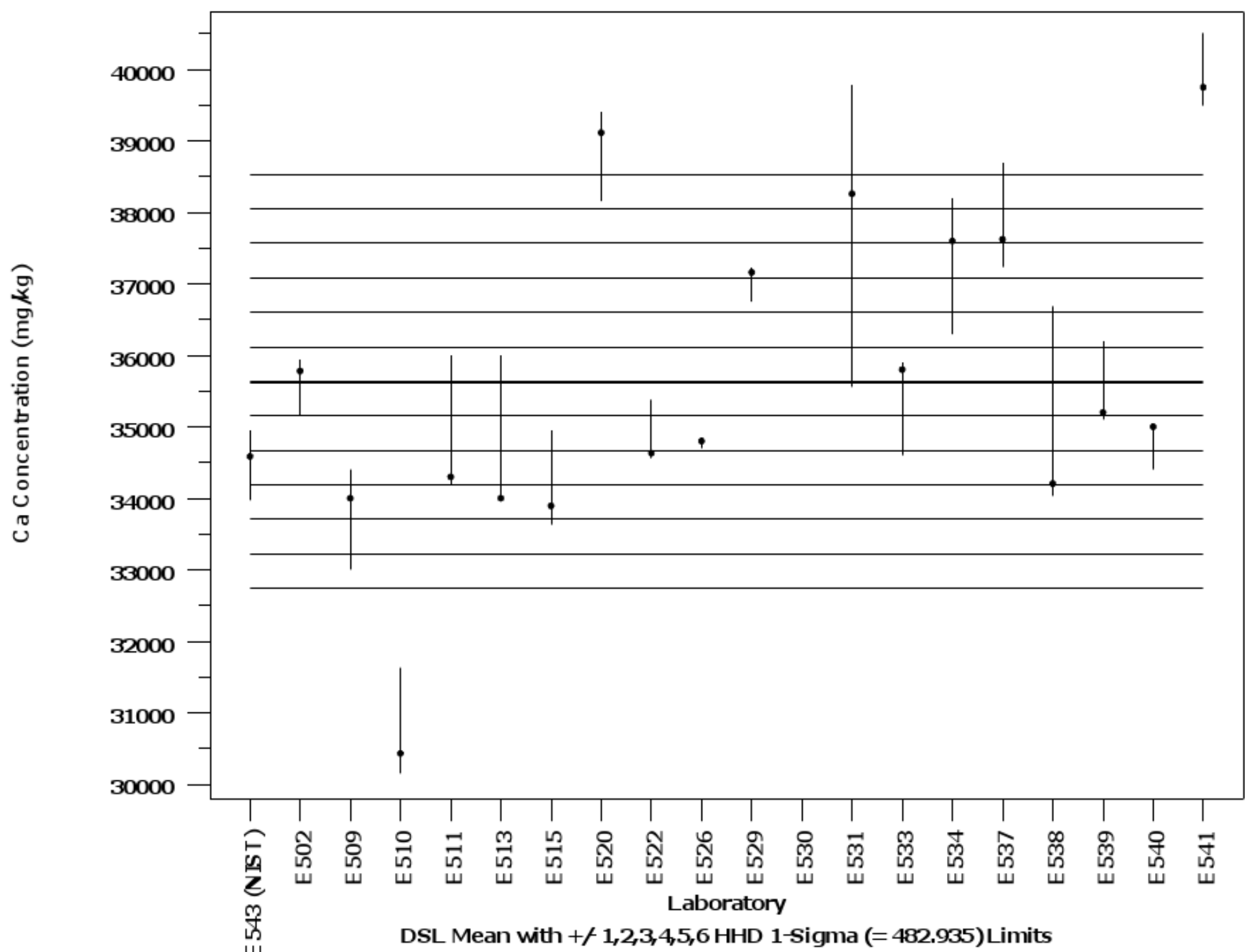




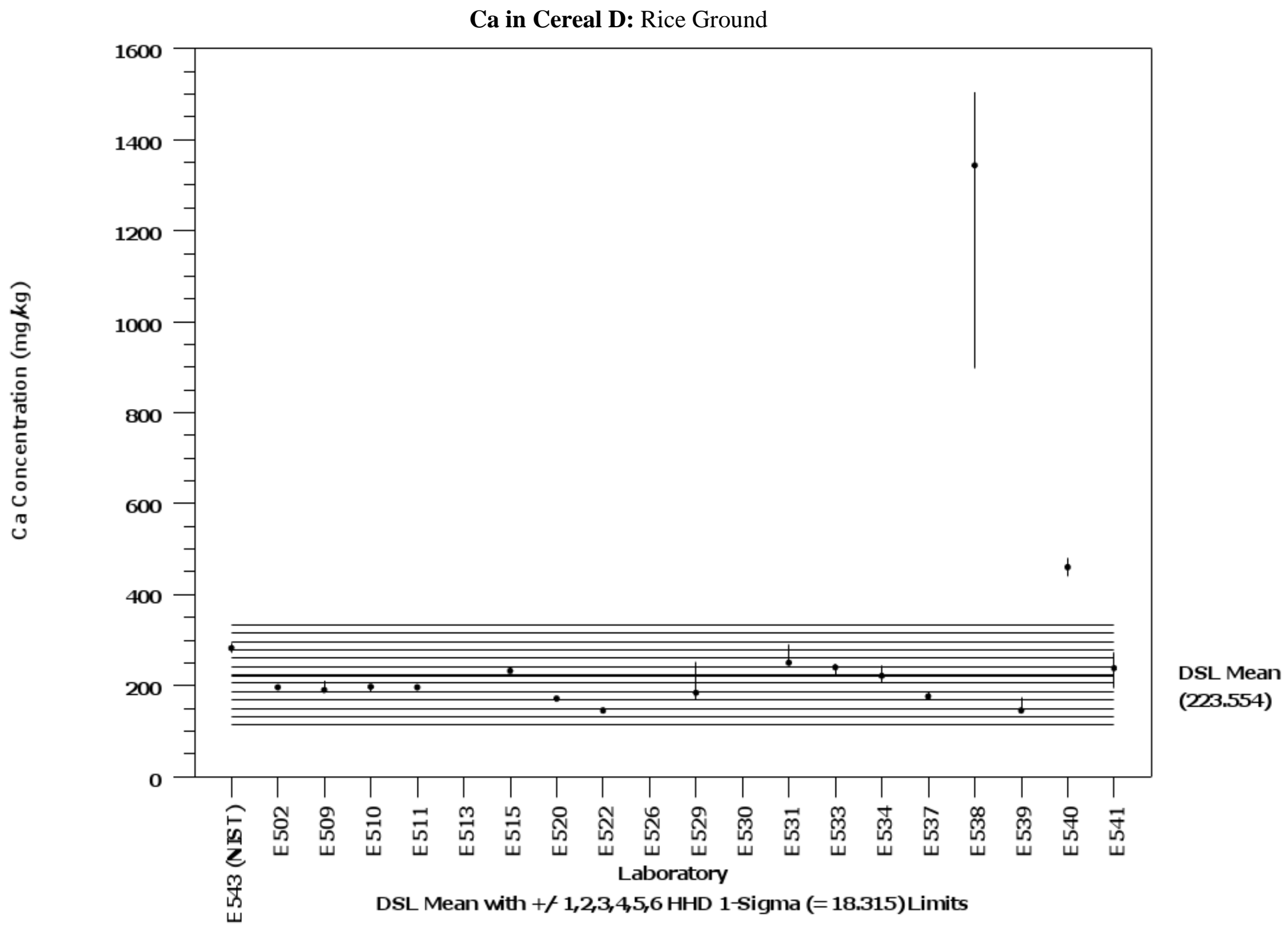


Ca in Cereal E: Rice Flake

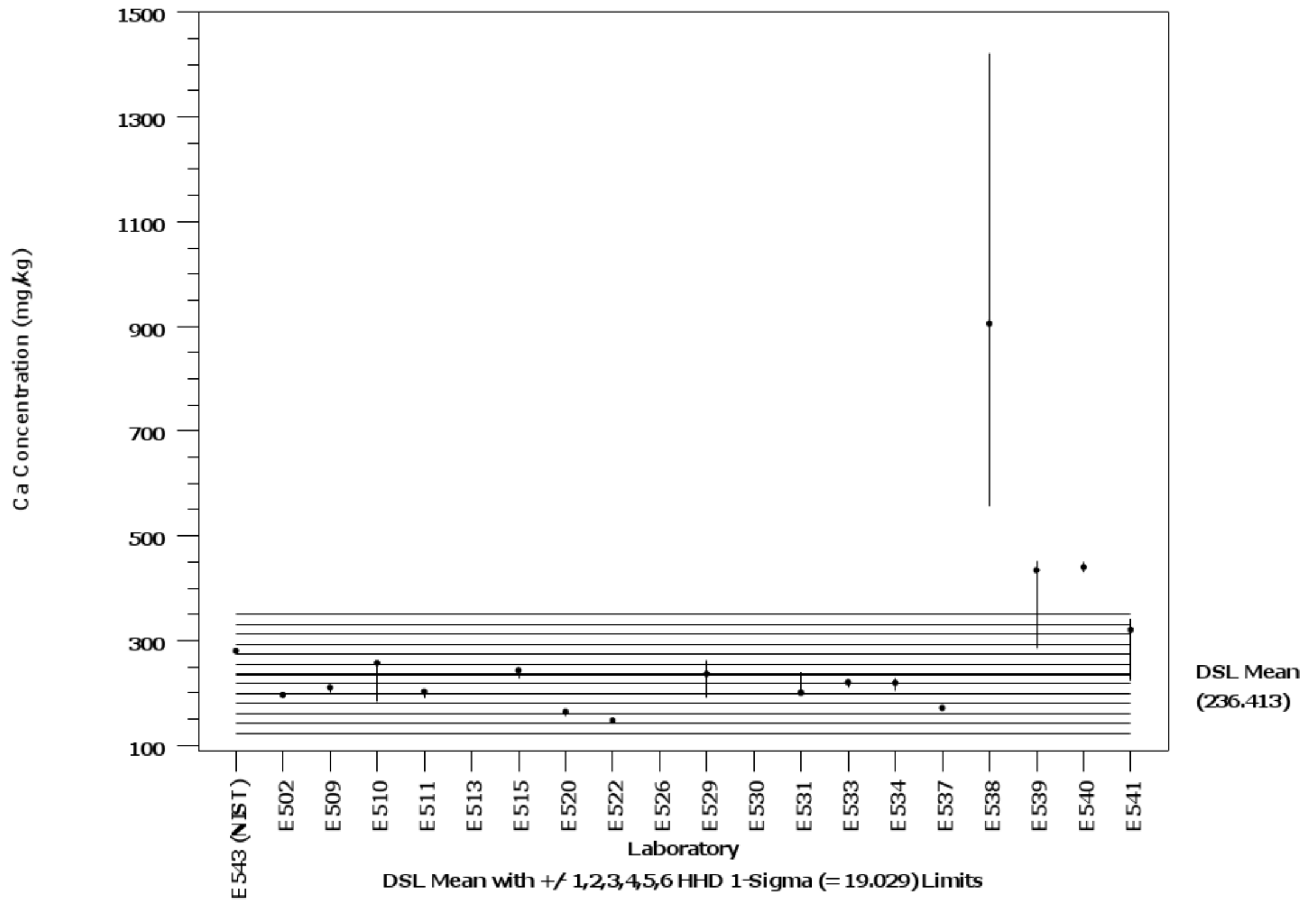


Ca in Cereal F: Wheat/Rice Crushed

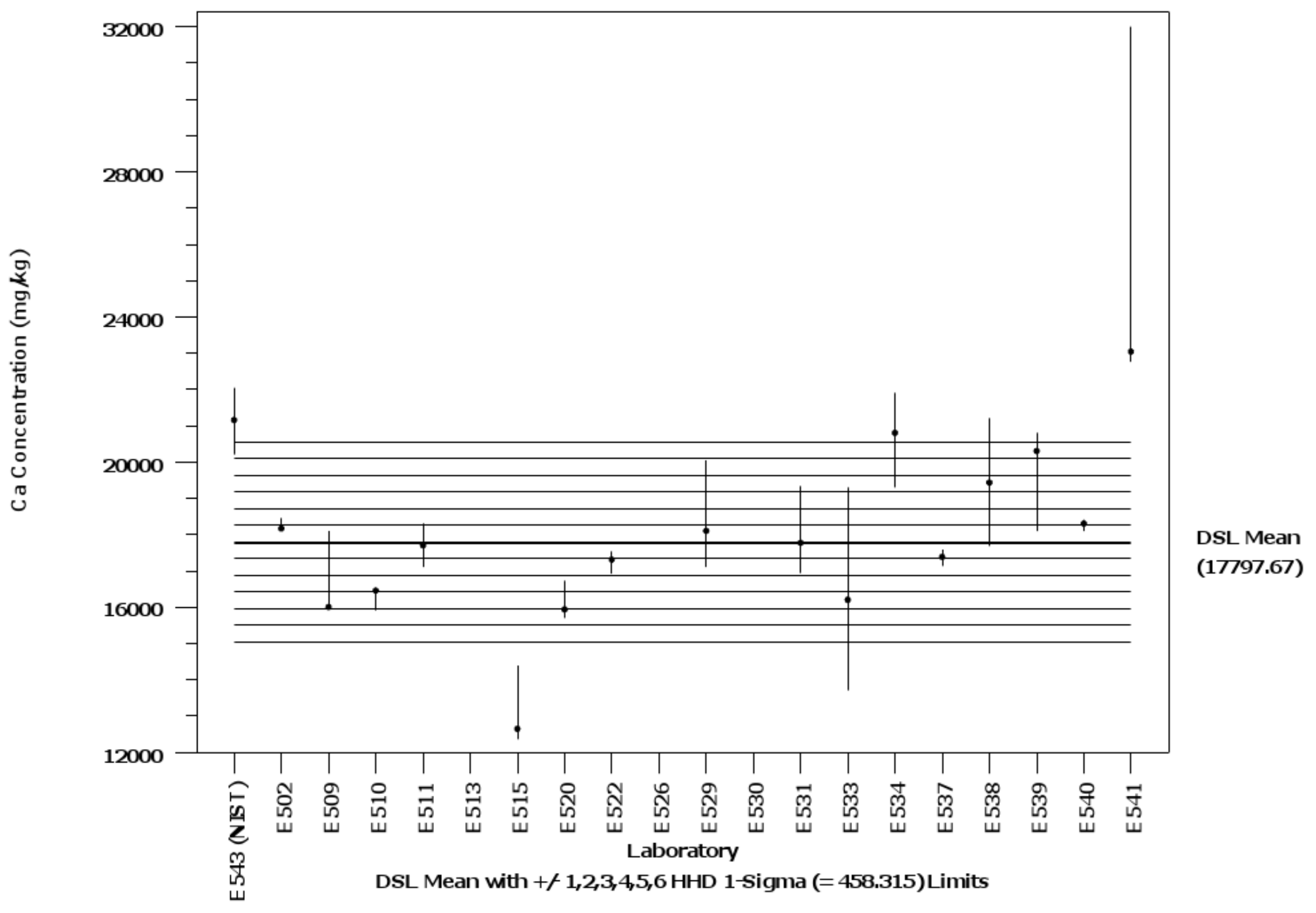


Fe in Cereal A: Wheat Ground (SRM 3233)

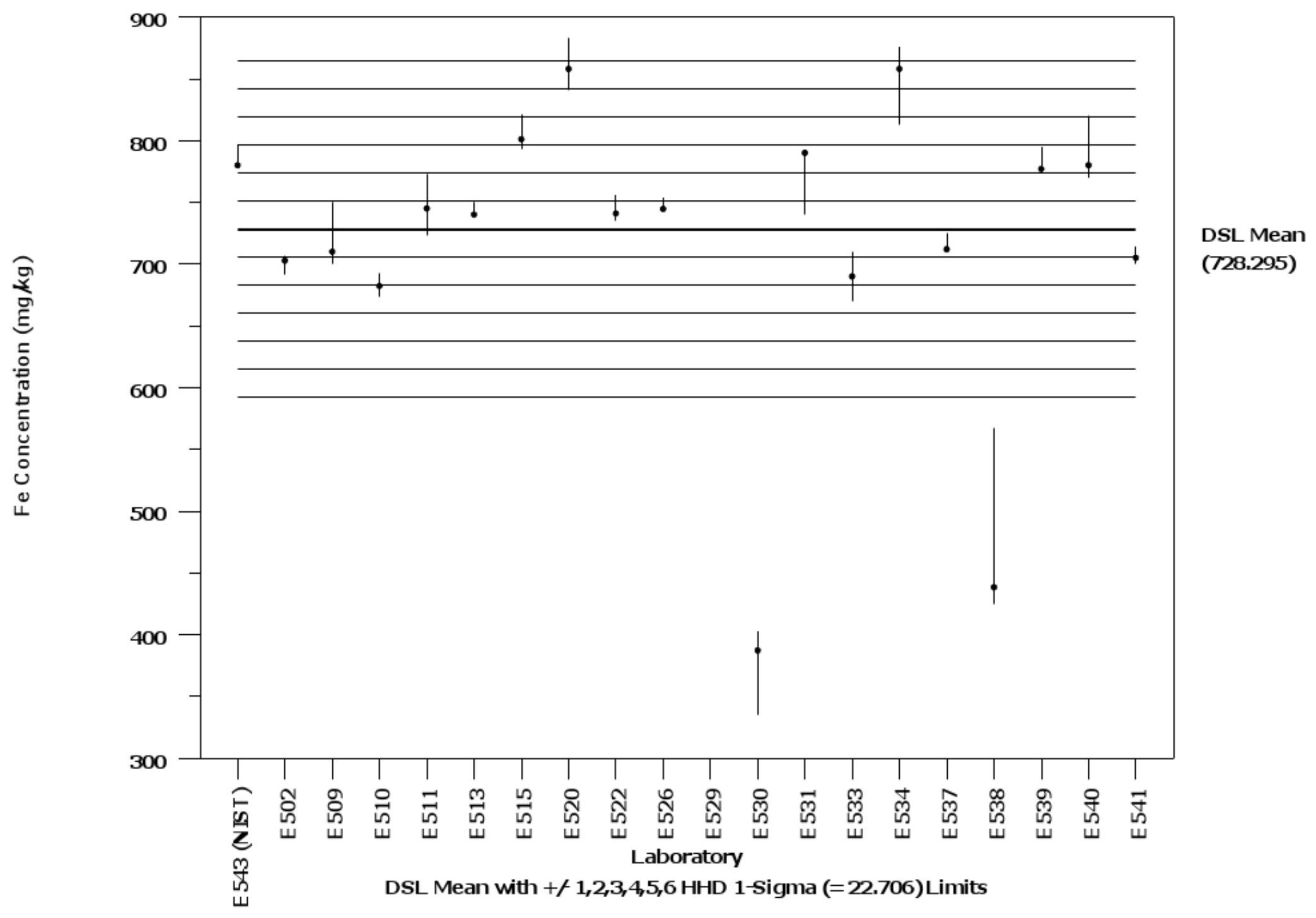




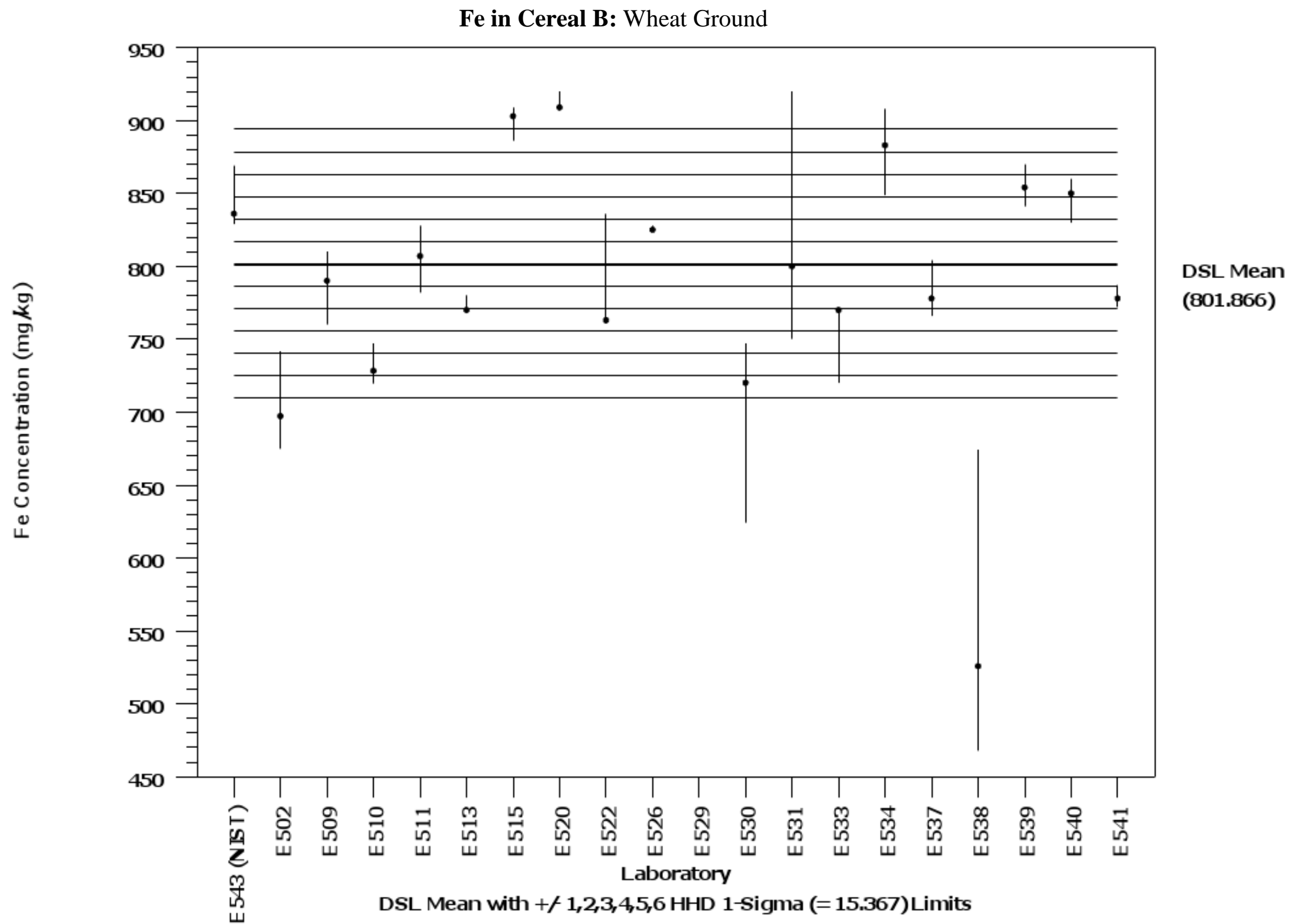


Fe in Cereal C: Wheat Flake

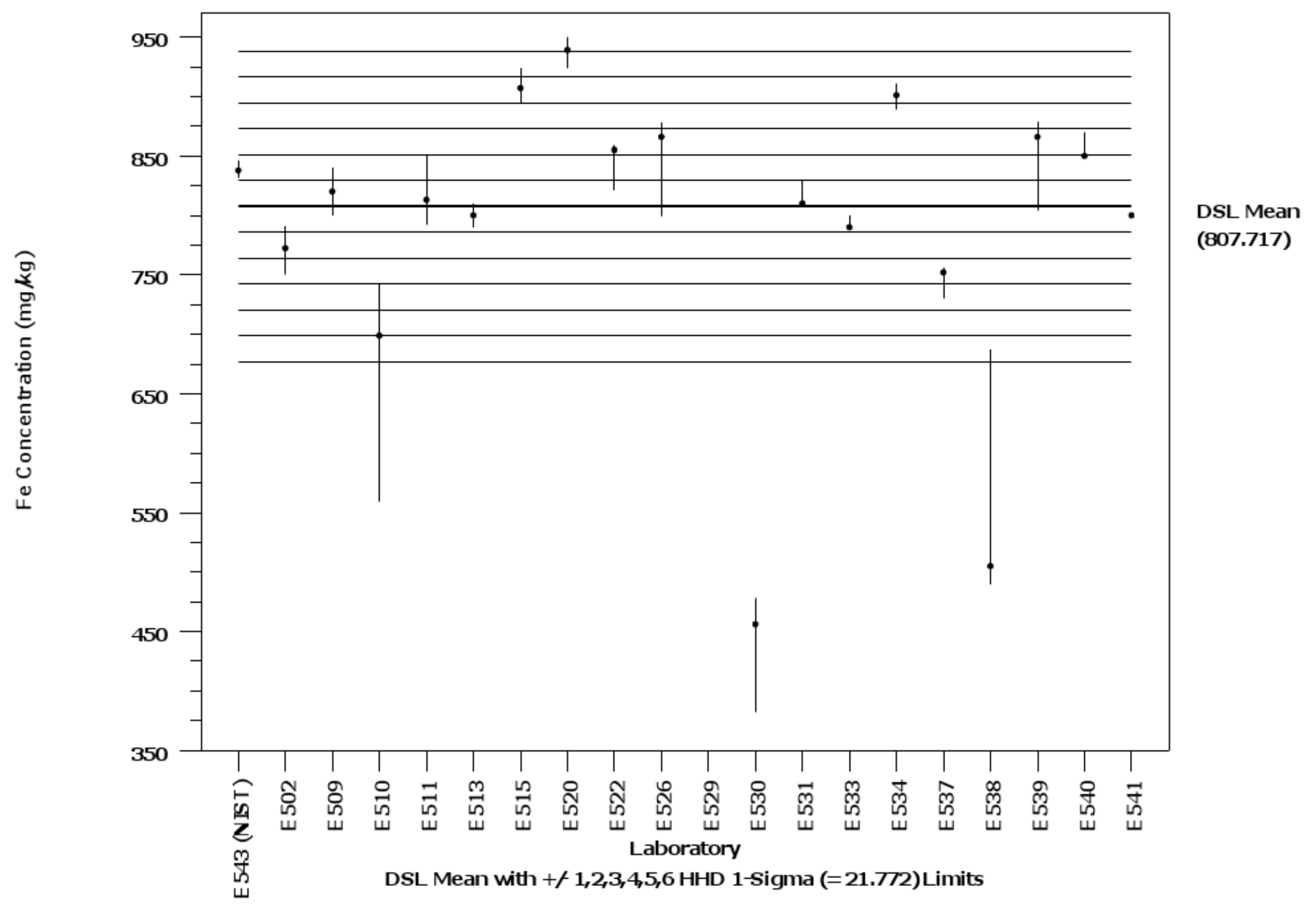




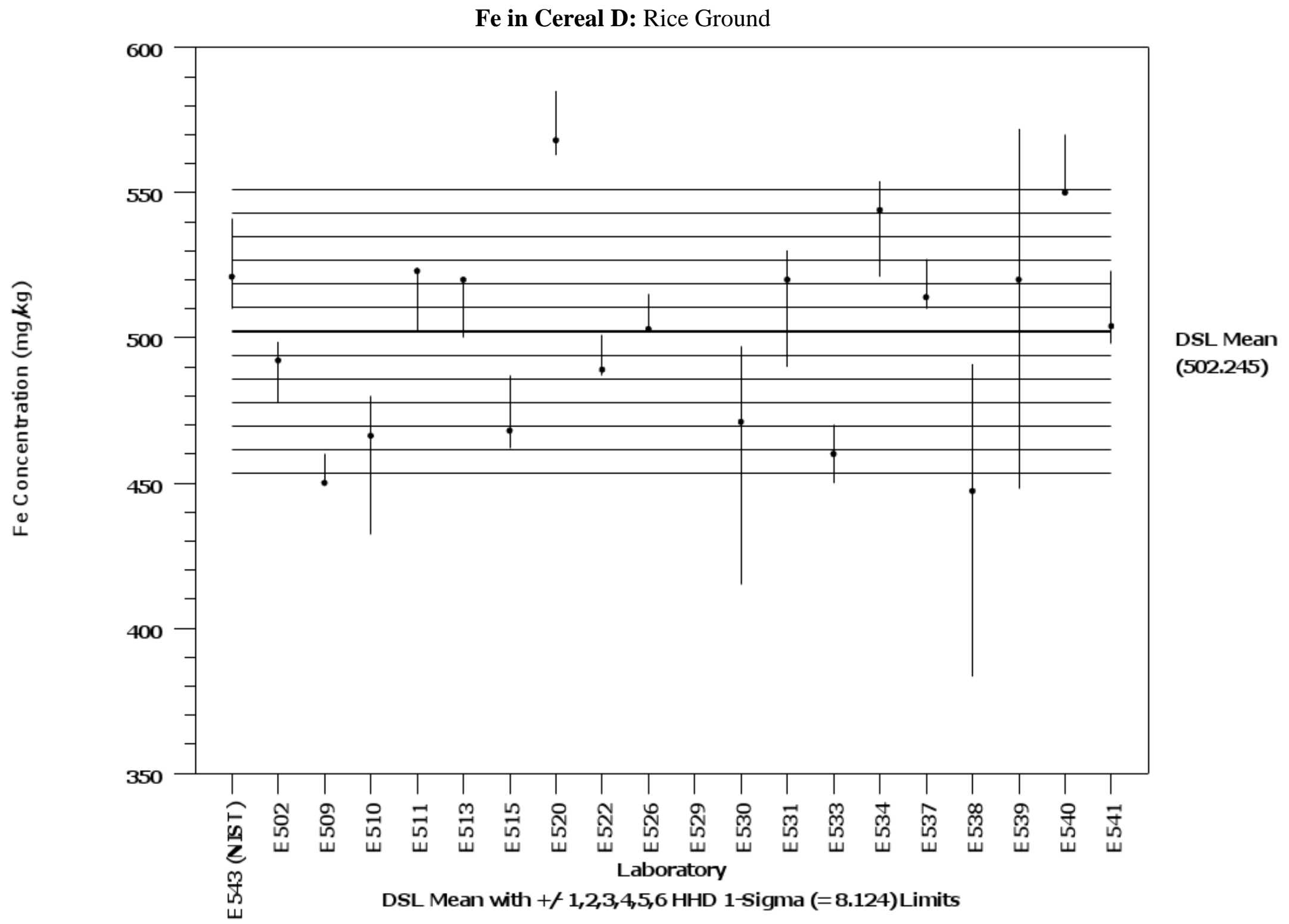




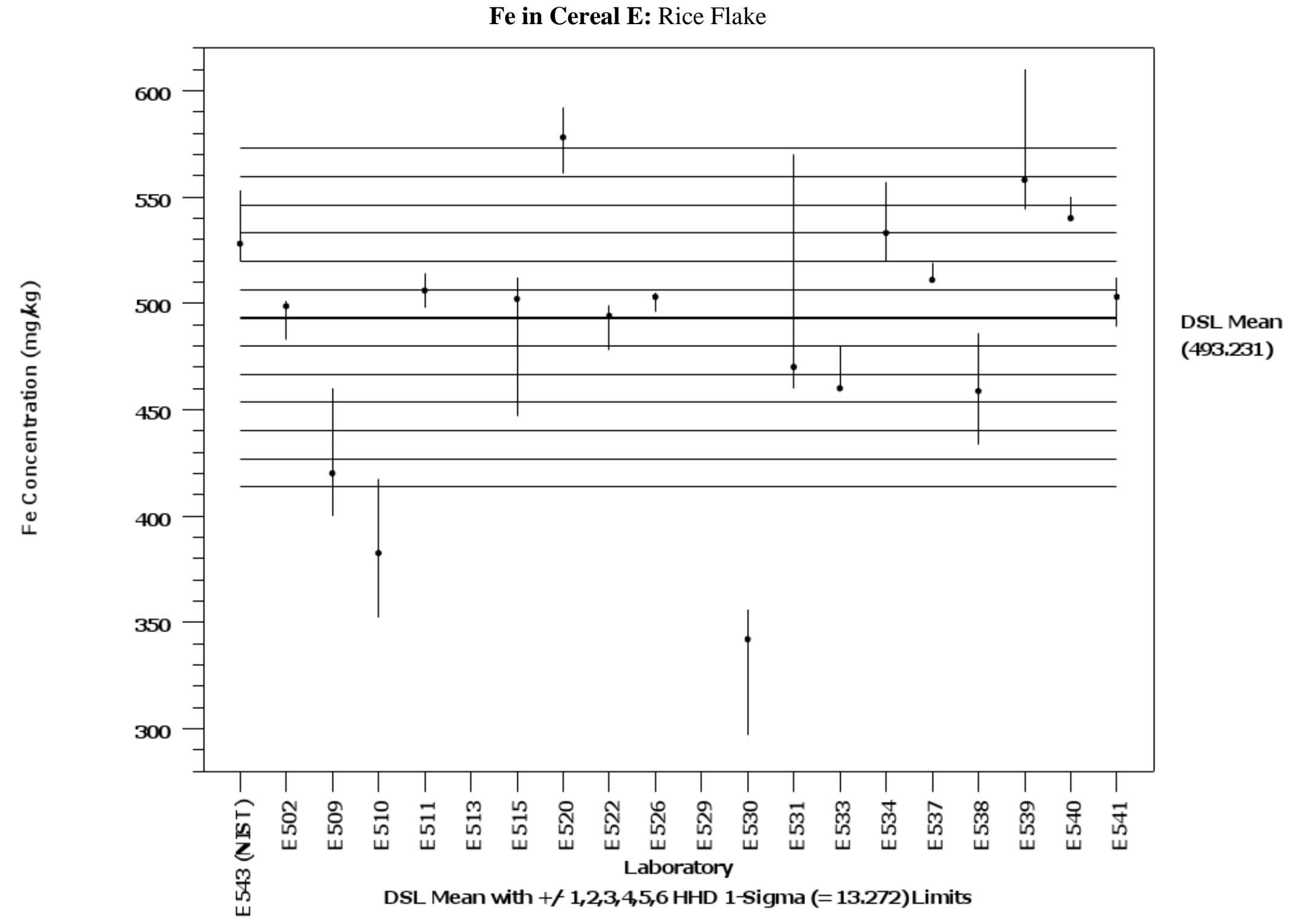




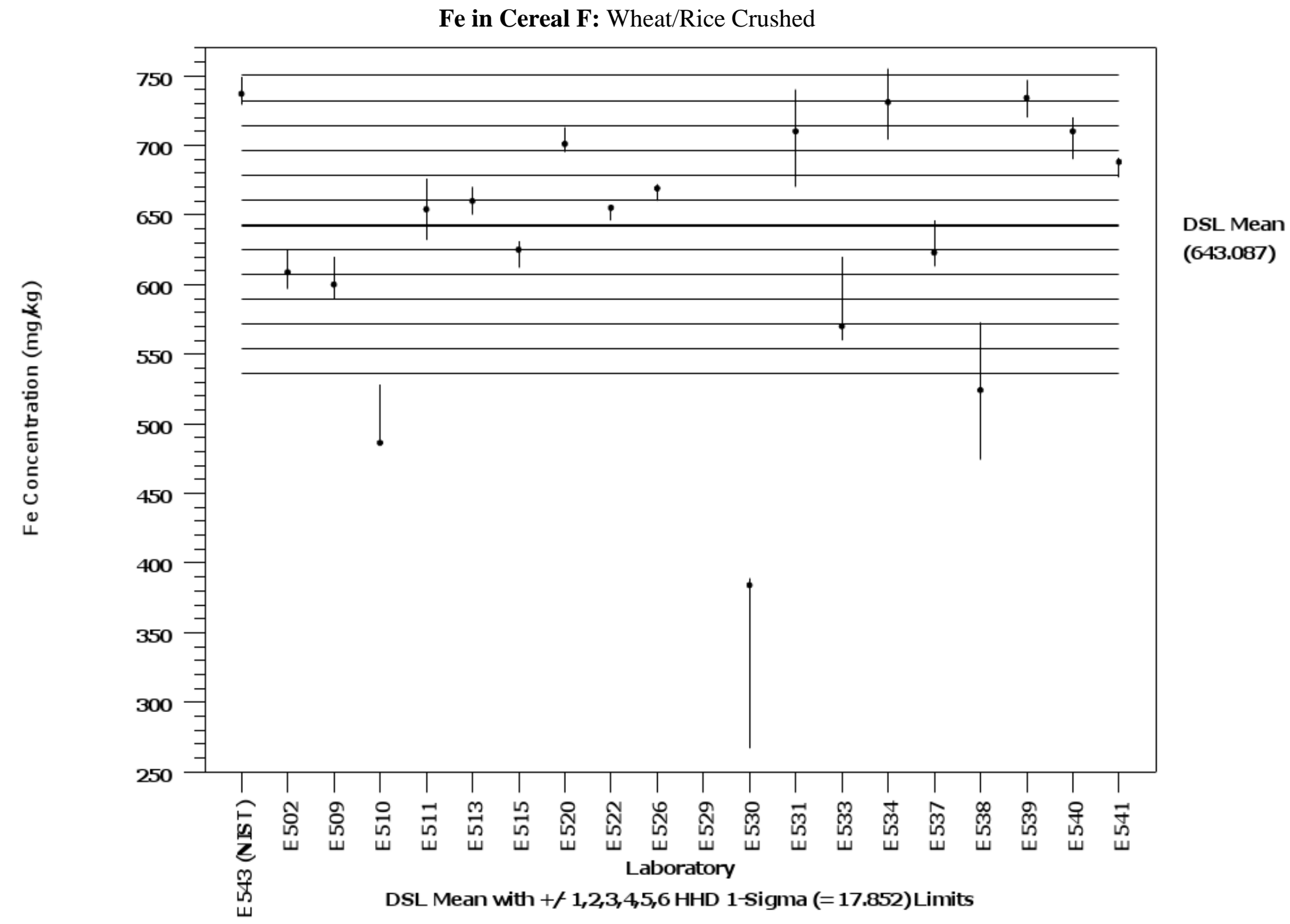




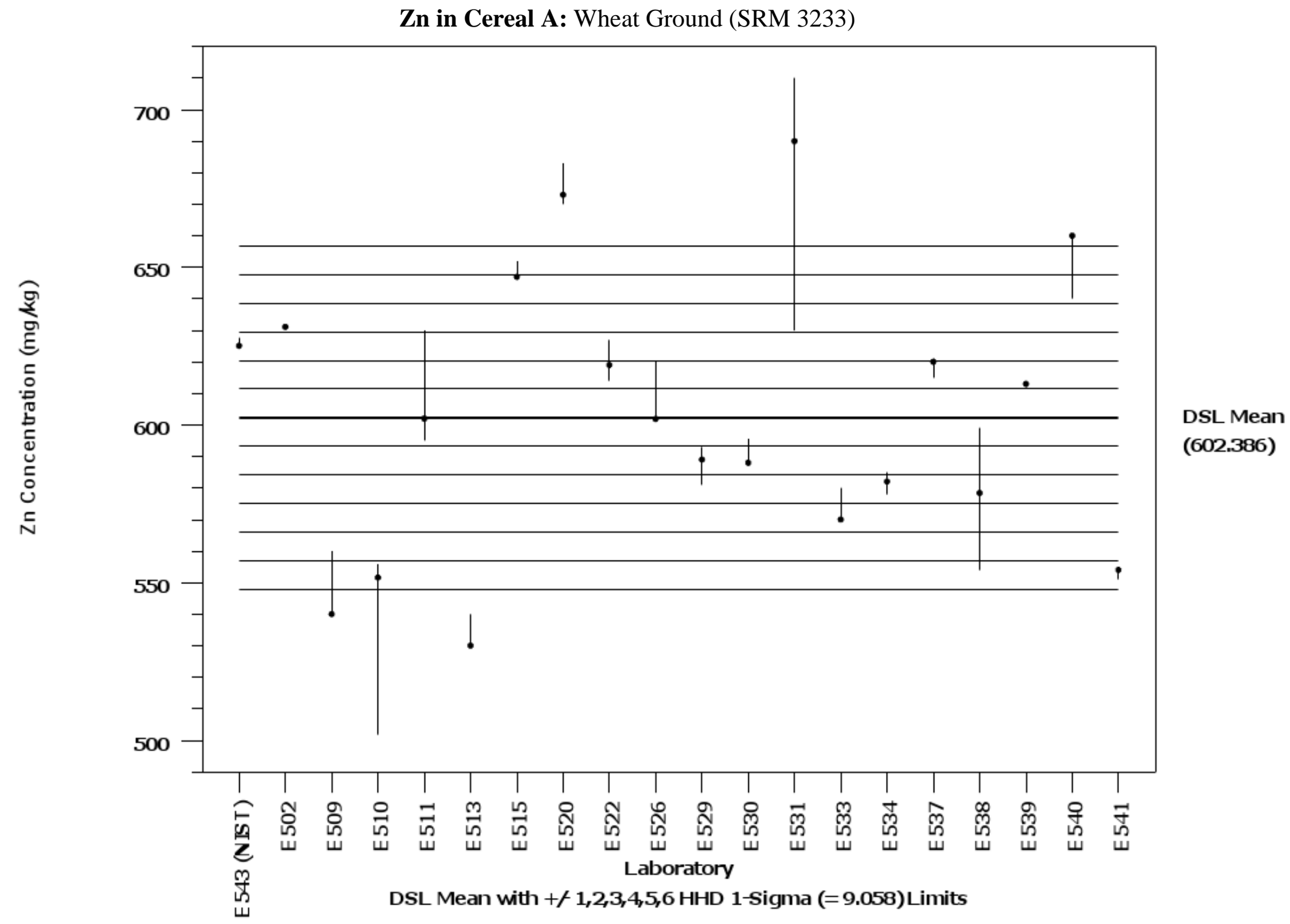




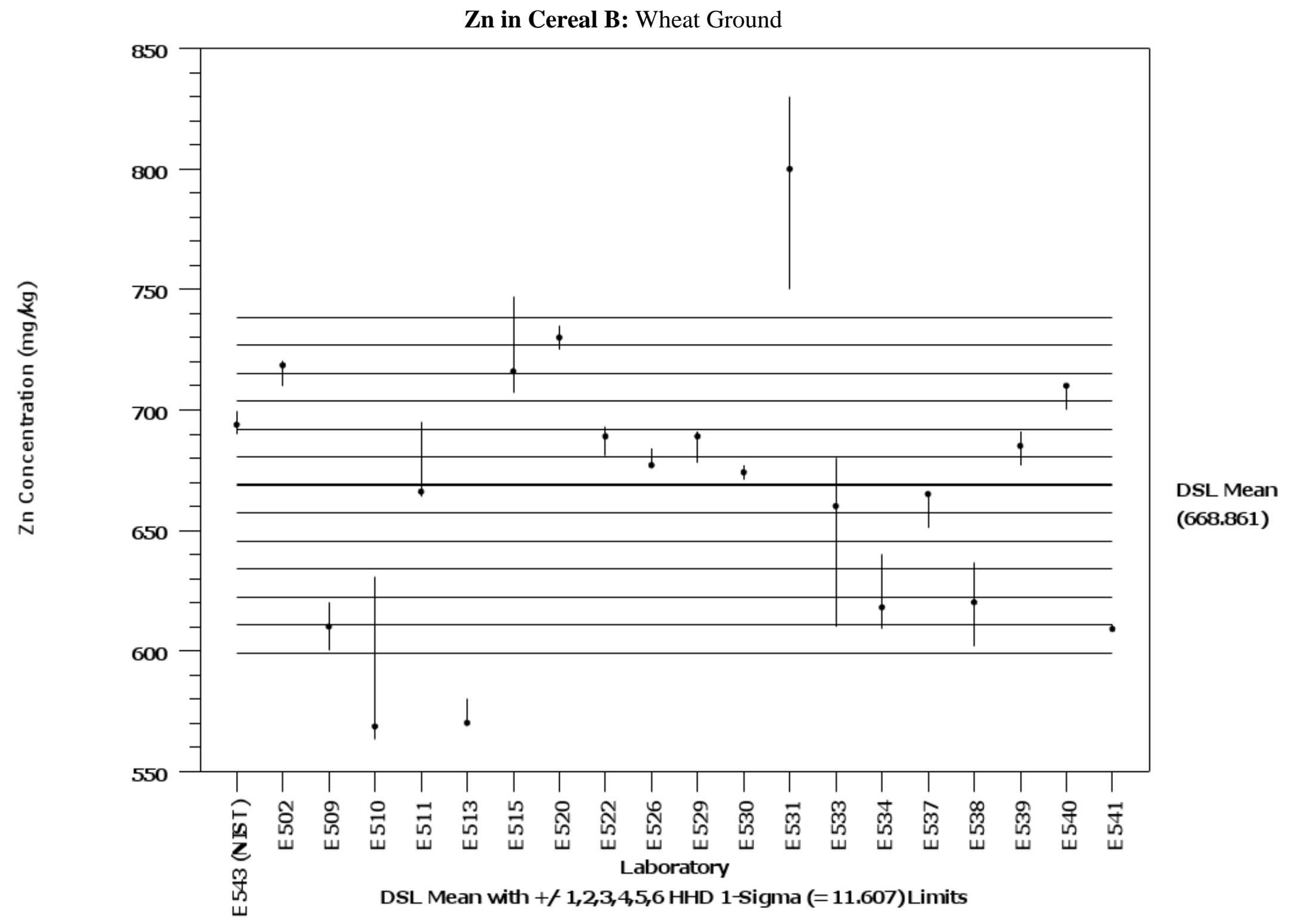




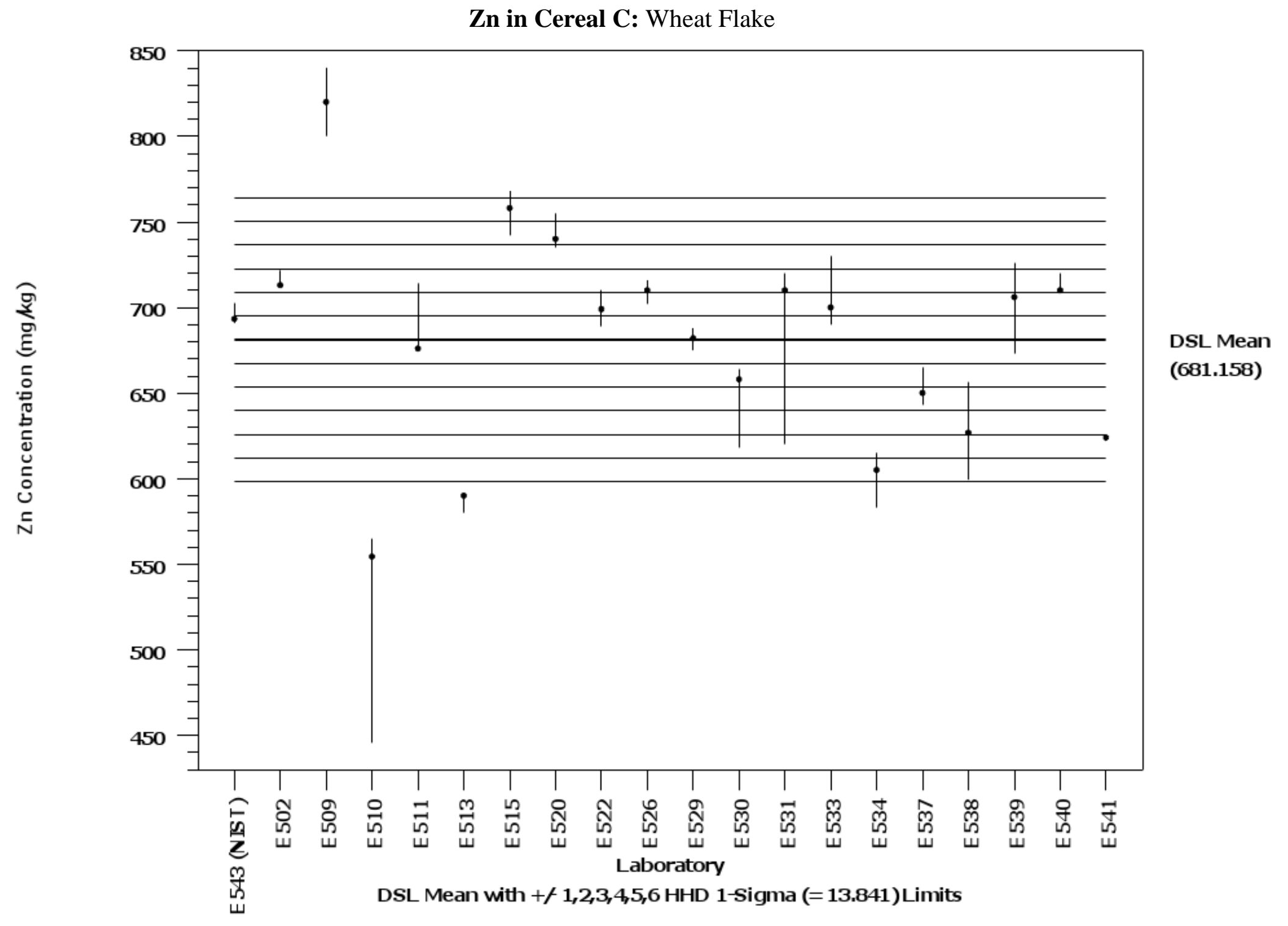




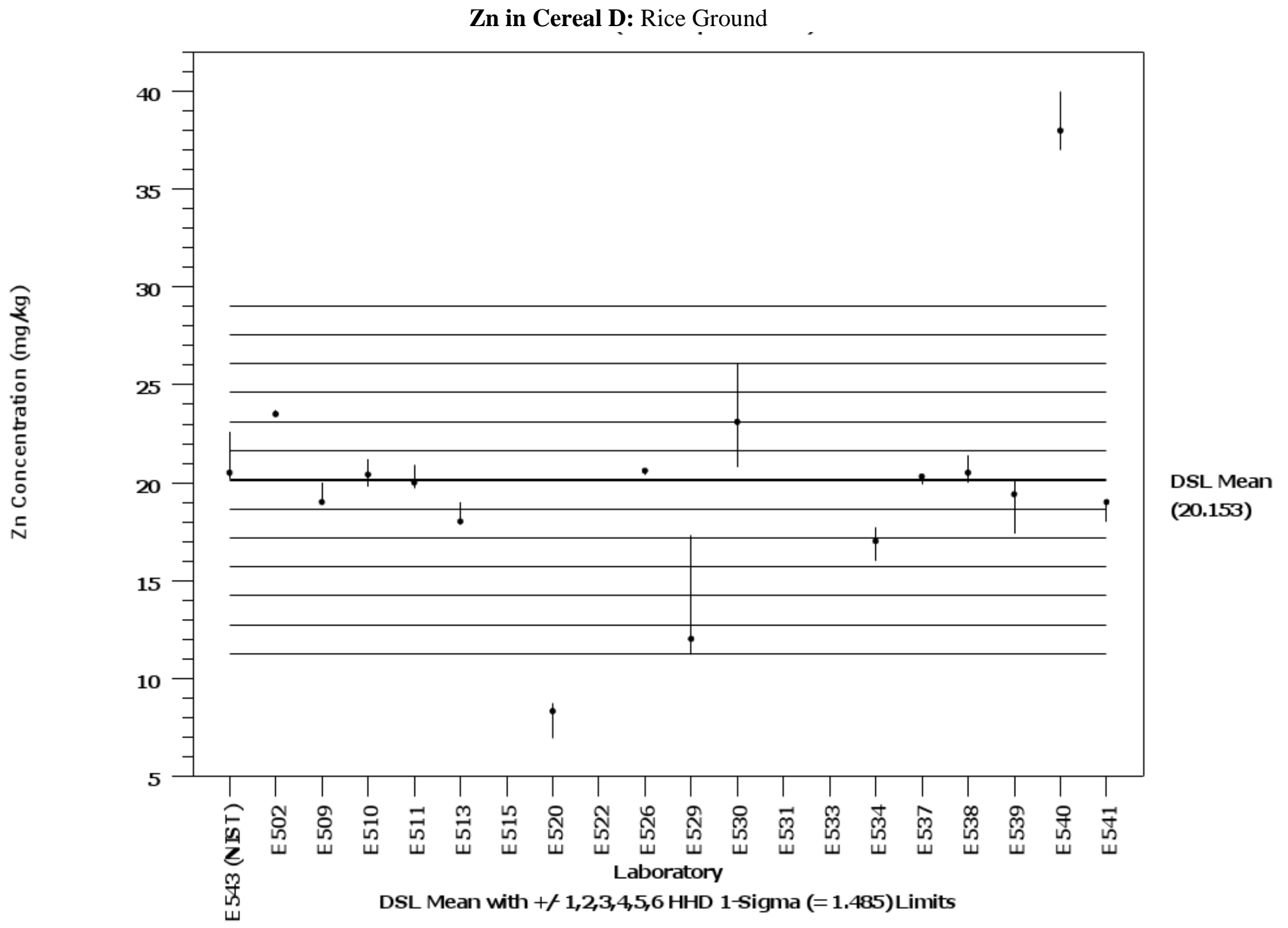




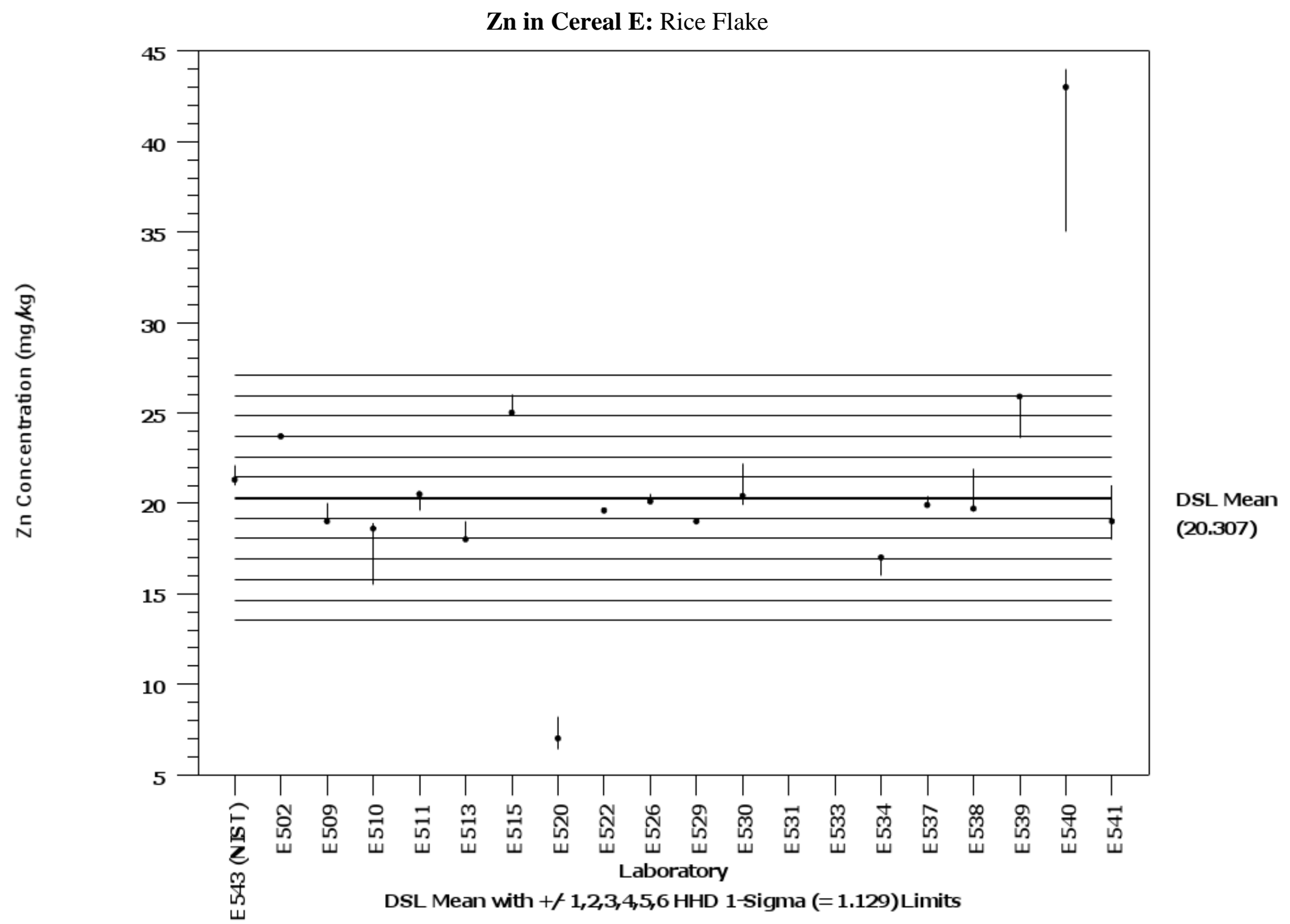




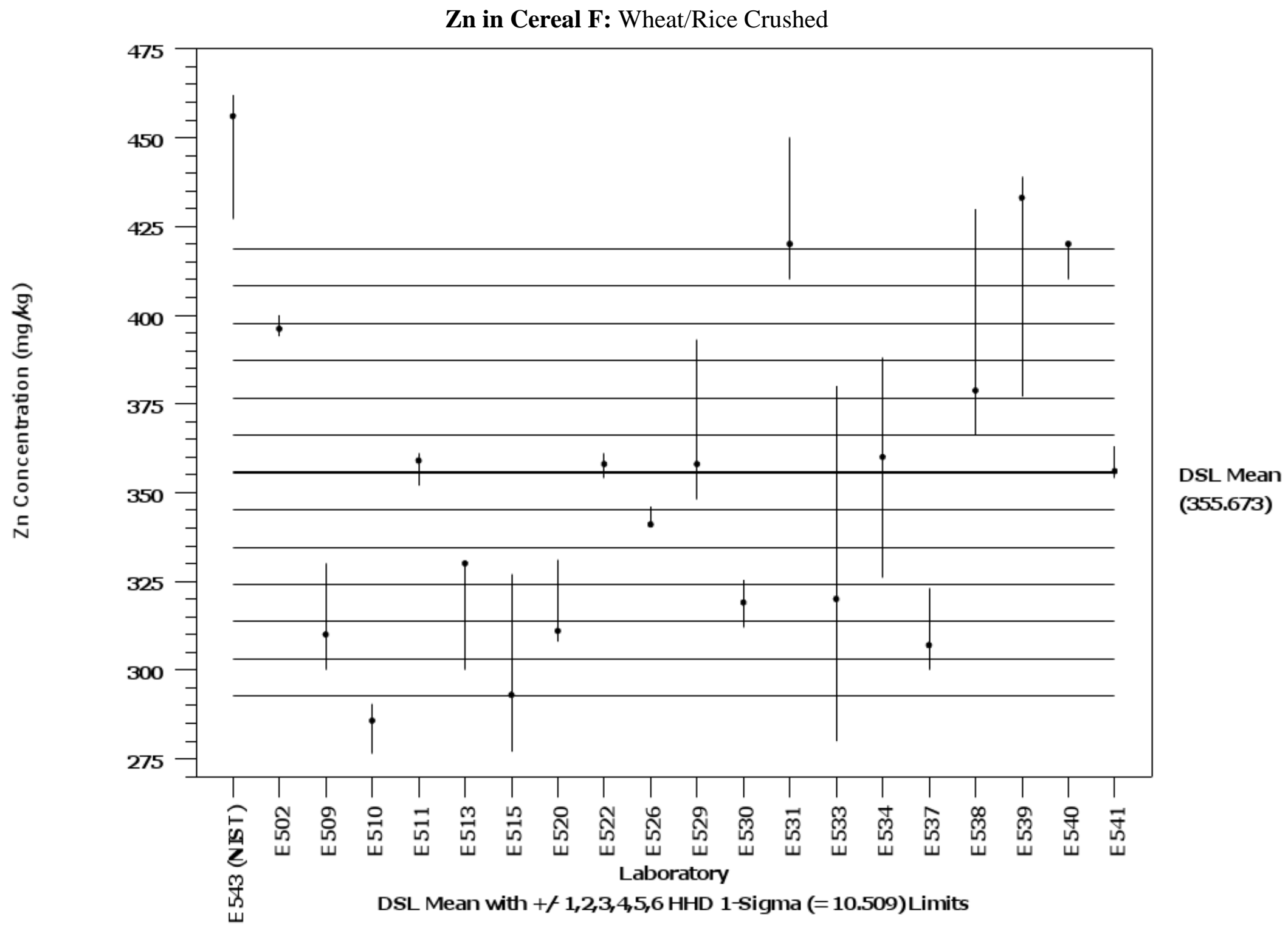




\section{APPENDIX E Individualized Laboratory Reports}

Appendix E contains an individualized summary of each of the 20 participating laboratories' performance in the interlaboratory exercise. Each laboratory was provided this 2-page laboratoryspecific report that delivered a table of each laboratory's summarized data and a summary consensus plot that graphically displays the laboratory's results in comparison to the exercise consensus results together with explanatory details and data interpretation. 


\section{Part II. Your Individual Laboratory Results (Lab Code: E543 - NIST)}

Your laboratory's data is summarized in Table Z. The mean (average) \pm 1 standard deviation were determined from the reported triplicate $(n=3)$ values for each of the 18 measurements $(\mathrm{Fe}, \mathrm{Ca}$ and Zn within the six study materials, Cereals A-F) in Exercise E.

Your laboratory's summary consensus plot for $\mathrm{Fe}$, $\mathrm{Ca}$ and $\mathrm{Zn}$ in the six cereal study materials is provided in Figure Z. The data have been normalized relative to consensus means and Horn-HornDuncan (HHD) uncertainties so that the consensus value is equal to zero. The thick blue line in this plot represents the consensus values for each of the 18 measurements (Fe, $\mathrm{Ca}$ and $\mathrm{Zn}$ within the six study materials, Cereals A-F). The thin blue lines represent \pm 3 HHD standard uncertainty intervals about the consensus values. The figure is scaled so that the top and bottom of the Y-axis represent \pm 6 HHD intervals. Your laboratory's results for each of the 18 measurements are illustrated by the black bars. The ends of bars that are entirely within the \pm 6 HHD intervals are marked with an open circle (०); red circles $(\bullet)$ indicate that some or all of the bar is outside the \pm 6 HHD interval.

The length of each bar indicates how well your measurements agree with themselves. The distance from the center of the bars to the consensus line indicates how well your measurements agree with those of the other participants in this study. (An example consensus plot with explanations is also included to aid in the interpretation your results.) As a general rule, we consider results that fall within \pm 3 HHD standard uncertainty lines to be within consensus (approximate $99 \%$ confidence limit) and results that have a black bar length $\approx 3$ HHD units are considered as acceptable precision. If values are consistently very high or low for a particular element, then a calibration problem may exist. In such cases, it is important to make sure your calibration standards are from a reliable source, the quality is confirmed, and that your calibration solutions are correctly prepared and stored. In addition, calibration points must be linear in the expected measurement range and closely surround expected values.

The means of your laboratory's measurement results for the wheat cereal (materials B \& C) and the rice cereal (D \& E) pairs agree well for all three elements, indicating a consistent sample preparation process. However, your results for Ca differ markedly between the wheat-based cereals (A, B \& C) and rice-based cereals (D \& E) which suggests that your Ca measurement process may have a matrix-specific bias. Your results for $\mathrm{Ca}$ and $\mathrm{Zn}$ are precise (bar lengths of 1 to $2 \mathrm{HHD}$ units), your results for Fe are somewhat less precise. Your results for all three elements in Cereal F are relatively high compared to those in the other materials. As discussed in Part I of the report, this hand-crushed material was noticeably less homogeneous than the other five materials.

Table Z. Summary of Fe, Ca and Zn values within the six study materials, Cereals A-F

\begin{tabular}{|c|cccccc|}
\hline Code: E543 & \multicolumn{5}{|c|}{ Mean Results \pm 1 SD (n= 3) } \\
\hline & $\begin{array}{c}\text { Cereal A } \\
\text { Wheat } \\
\text { Ground } \\
\text { (SRM 3233) }\end{array}$ & $\begin{array}{c}\text { Cereal B } \\
\text { Wheat } \\
\text { Ground }\end{array}$ & $\begin{array}{c}\text { Cereal C } \\
\text { Wheat } \\
\text { Flake }\end{array}$ & $\begin{array}{c}\text { Cereal D } \\
\text { Rice } \\
\text { Ground }\end{array}$ & $\begin{array}{c}\text { Cereal E } \\
\text { Rice } \\
\text { Flake }\end{array}$ & $\begin{array}{c}\text { Cereal F } \\
\text { Wheat/Rice } \\
\text { Crushed }\end{array}$ \\
\hline $\mathrm{Fe}(\mu \mathrm{g} / \mathrm{g})$ & $785 \pm 10$ & $845 \pm 21$ & $839 \pm 7$ & $524 \pm 16$ & $534 \pm 17$ & $738 \pm 10$ \\
$\mathrm{Ca}(\mu \mathrm{g} / \mathrm{g})$ & $37250 \pm 340$ & $34620 \pm 160$ & $34500 \pm 500$ & $282 \pm 11$ & $279 \pm 5$ & $21130 \pm 920$ \\
$\mathrm{Zn}(\mu \mathrm{g} / \mathrm{g})$ & $626 \pm 2$ & $694 \pm 5$ & $696 \pm 6$ & $21 \pm 1$ & $21 \pm 1$ & $448 \pm 19$ \\
\hline
\end{tabular}


Figure Z. A consensus plot summarizing your individual results: (Lab Code: E543 - NIST)

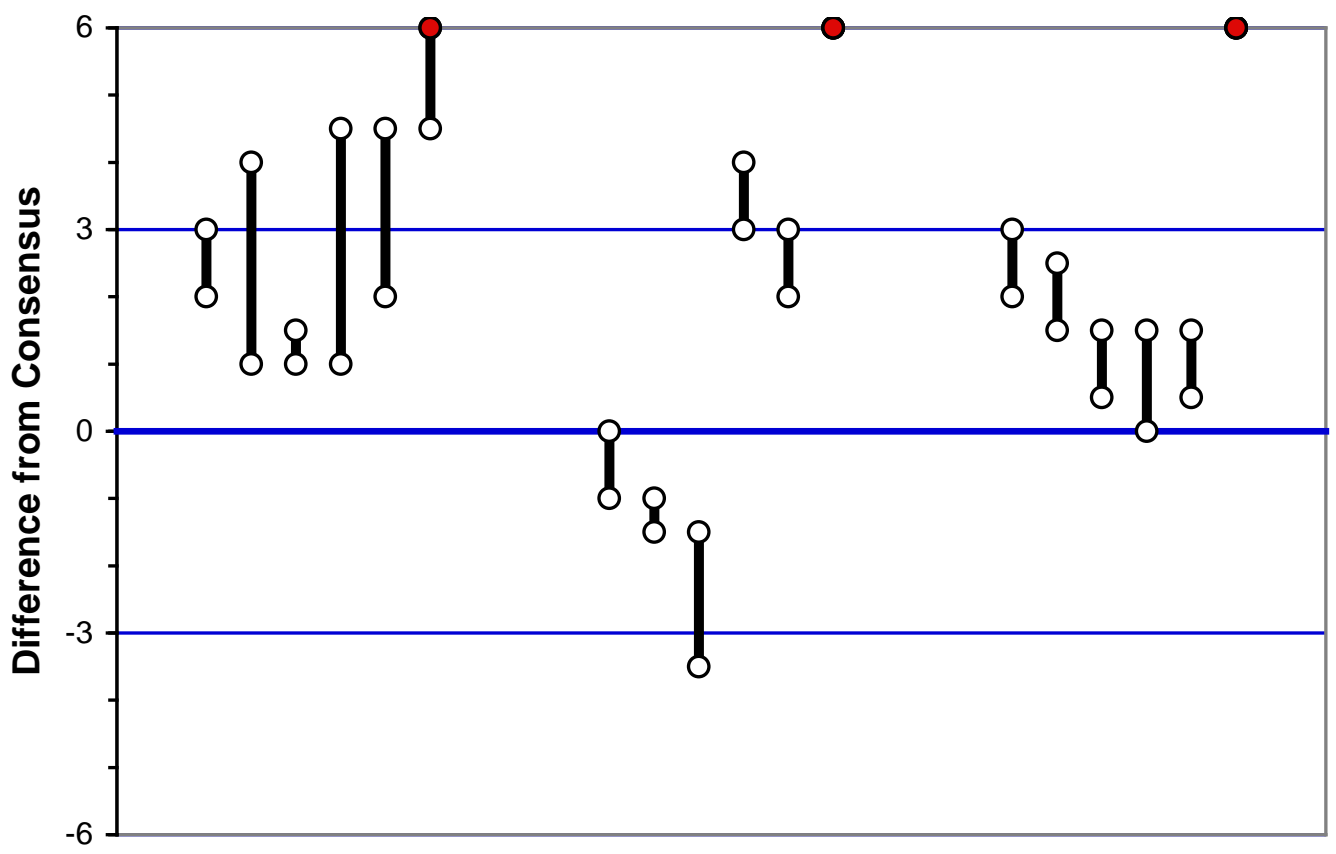
Cereal: A B C D E F Element:
$\mathrm{Fe}$
A B C D E F
$\mathrm{Ca}$

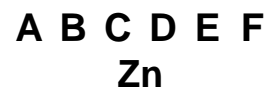

How to interpret your consensus summary plot:

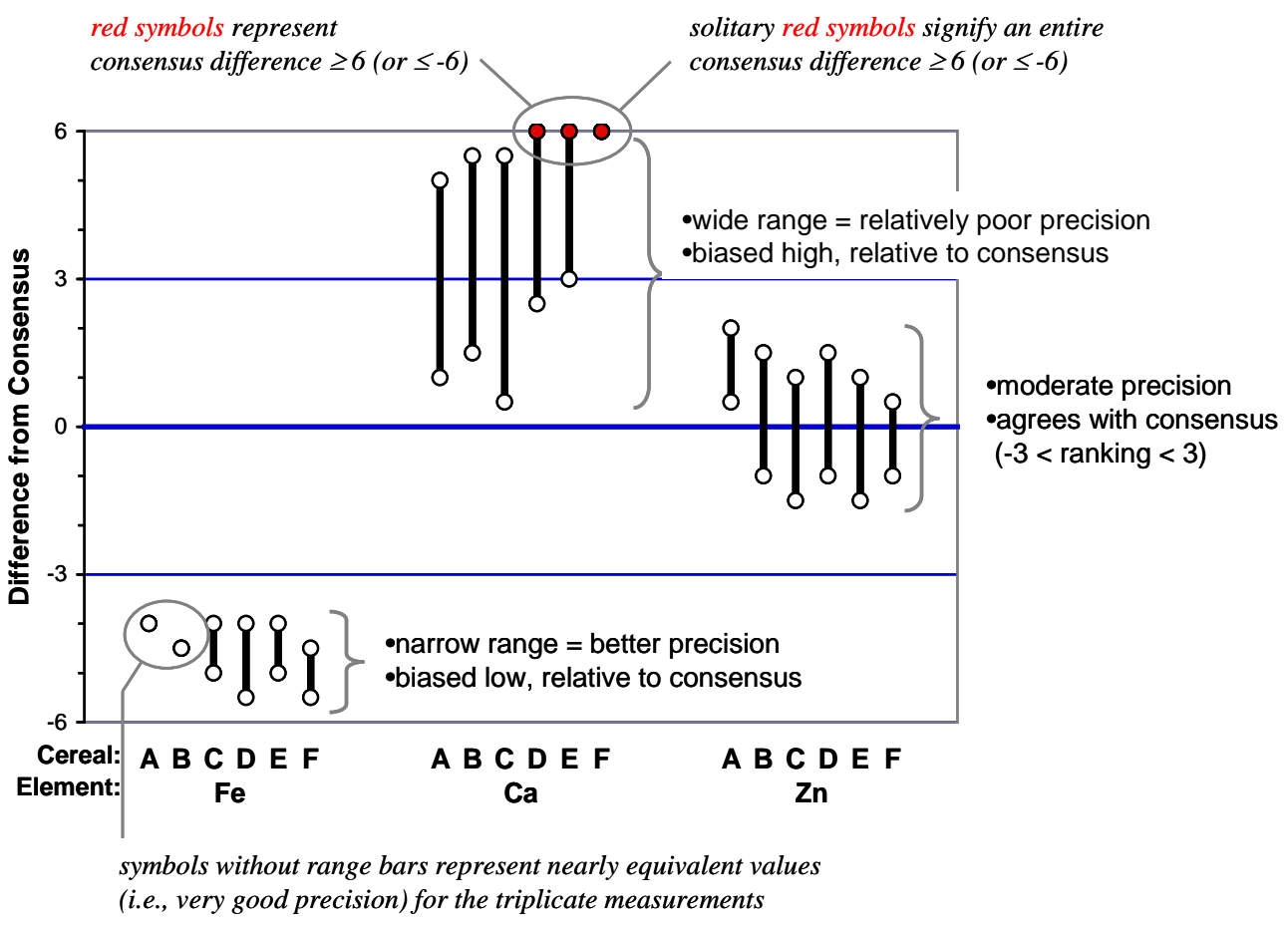




\section{Part II. Your Individual Laboratory Results (Lab Code: E502)}

Your laboratory's data is summarized in Table Z $\mathbf{Z}$. The mean (average) \pm 1 standard deviation were determined from the reported triplicate $(n=3)$ values for each of the 18 measurements $(\mathrm{Fe}, \mathrm{Ca}$ and Zn within the six study materials, Cereals A-F) in Exercise E.

Your laboratory's summary consensus plot for Fe, Ca and $\mathrm{Zn}$ in the six cereal study materials is provided in Figure $\mathbf{Z}$. The data have been normalized relative to consensus means and Horn-HornDuncan (HHD) uncertainties so that the consensus value is equal to zero. The thick blue line in this plot represents the consensus values for each of the 18 measurements (Fe, $\mathrm{Ca}$ and $\mathrm{Zn}$ within the six study materials, Cereals A-F). The thin blue lines represent \pm 3 HHD standard uncertainty intervals about the consensus values. The figure is scaled so that the top and bottom of the Y-axis represent \pm 6 HHD intervals. Your laboratory's results for each of the 18 measurements are illustrated by the black bars. The ends of bars that are entirely within the \pm 6 HHD intervals are marked with an open circle (०); red circles $(\bullet)$ indicate that some or all of the bar is outside the \pm 6 HHD interval.

The length of each bar indicates how well your measurements agree with themselves. The distance from the center of the bars to the consensus line indicates how well your measurements agree with those of the other participants in this study. (An example consensus plot with explanations is also included to aid in the interpretation your results.) As a general rule, we consider results that fall within \pm 3 HHD standard uncertainty lines to be within consensus (approximate 99\% confidence limit) and results that have a black bar length $\approx 3$ HHD units are considered as acceptable precision. If values are consistently very high or low for a particular element, then a calibration problem may exist. In such cases, it is important to make sure your calibration standards are from a reliable source, the quality is confirmed and that your calibration solutions are correctly prepared and stored. In addition, calibration points must be linear in the expected measurement range and closely surround expected values.

Your laboratory’s measurement results for Ca agree well with the consensus values; your Zn values are consistently somewhat greater than consensus and those for Fe are a bit lower. You may want to check the calibration materials you use for Fe and $\mathrm{Zn}$. The means of your results for the wheatbased cereal (B \& C) and the rice-based cereal (D \& E) pairs agree well for Ca and Zn, indicating a consistent sample preparation process. Your results for $\mathrm{Ca}$ and $\mathrm{Zn}$ are precise (bar length of about 1 HHD units), your results for Fe are somewhat less so. The very low result for Fe in material B stands out suggesting possible problems with the linearity of your calibration curve. Based on the information given to the DSQAP Team, the range of your calibration curve extends quite high which would cause possible saturation at the upper points of the curve leading to unreliable results.

Table Z. Summary of Fe, Ca and Zn values within the six study materials, Cereals A-F

\begin{tabular}{|c|cccccc|}
\hline Lab Code: E502 & \multicolumn{5}{|c|}{ Mean Results $\pm \mathbf{1}$ SD (n= 3) } \\
\hline & $\begin{array}{c}\text { Cereal A } \\
\text { Wheat } \\
\text { Ground } \\
\text { SRM 3233) }\end{array}$ & $\begin{array}{c}\text { Cereal B } \\
\text { Wheat } \\
\text { Ground }\end{array}$ & $\begin{array}{c}\text { Cereal C } \\
\text { Wheat } \\
\text { Flake }\end{array}$ & $\begin{array}{c}\text { Cereal D } \\
\text { Rice } \\
\text { Ground }\end{array}$ & $\begin{array}{c}\text { Cereal E } \\
\text { Rice } \\
\text { Flake }\end{array}$ & $\begin{array}{c}\text { Cereal F } \\
\text { Wheat/Rice } \\
\text { Crushed }\end{array}$ \\
\hline $\mathrm{Fe}(\mu \mathrm{g} / \mathrm{g})$ & $700 \pm 8$ & $704 \pm 34$ & $771 \pm 21$ & $489 \pm 11$ & $494 \pm 10$ & $610 \pm 14$ \\
$\mathrm{Ca}(\mu \mathrm{g} / \mathrm{g})$ & $37330 \pm 200$ & $35760 \pm 260$ & $35630 \pm 410$ & $196 \pm 1$ & $197 \pm 5$ & $18260 \pm 170$ \\
$\mathrm{Zn}(\mu \mathrm{g} / \mathrm{g})$ & $631 \pm 1$ & $716 \pm 6$ & $716 \pm 5$ & $24 \pm 0.1$ & $24 \pm 0.1$ & $397 \pm 3$ \\
\hline
\end{tabular}


Figure Z. A consensus plot summarizing your individual results (Lab Code: E502)

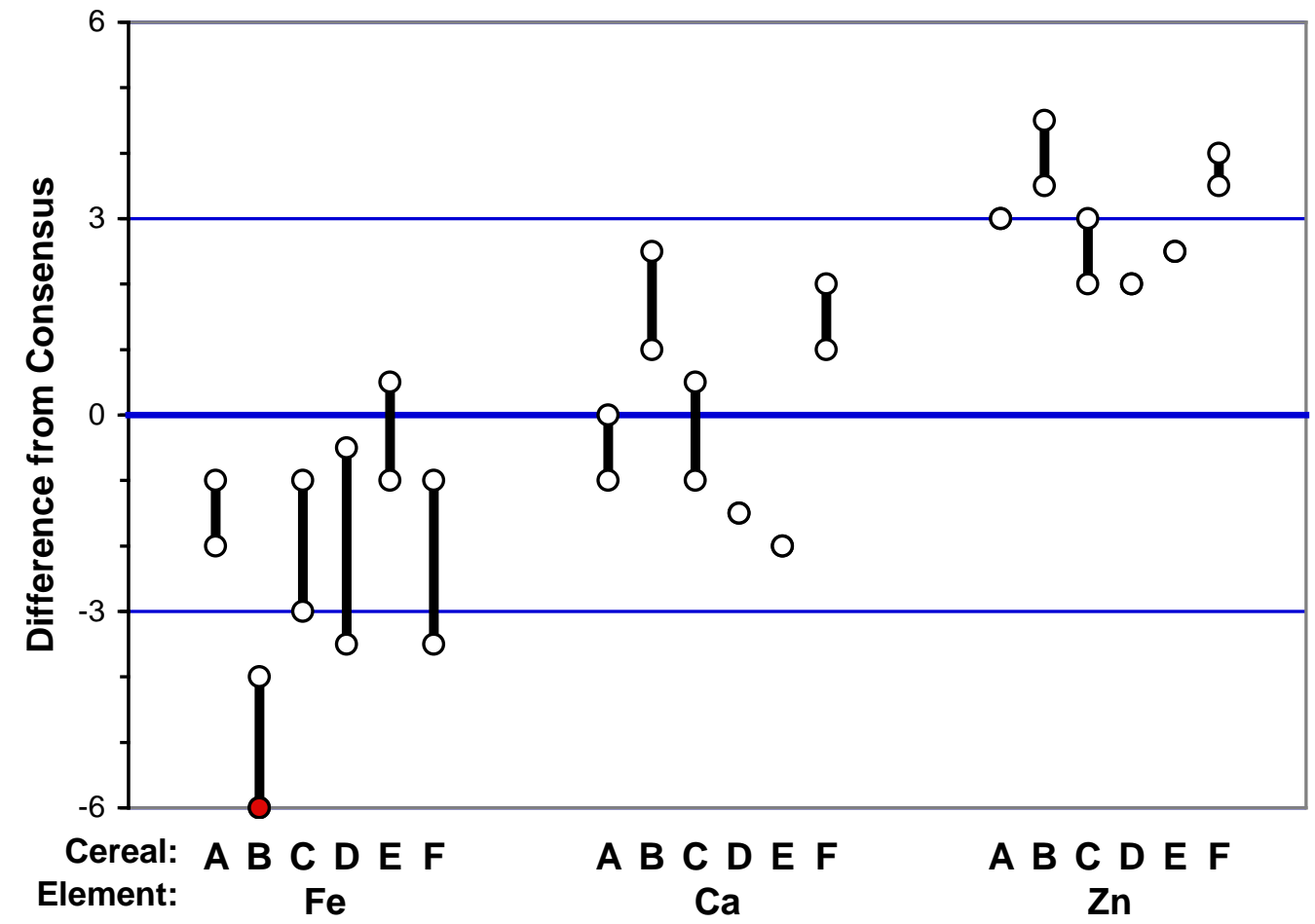

How to interpret your consensus summary plot:

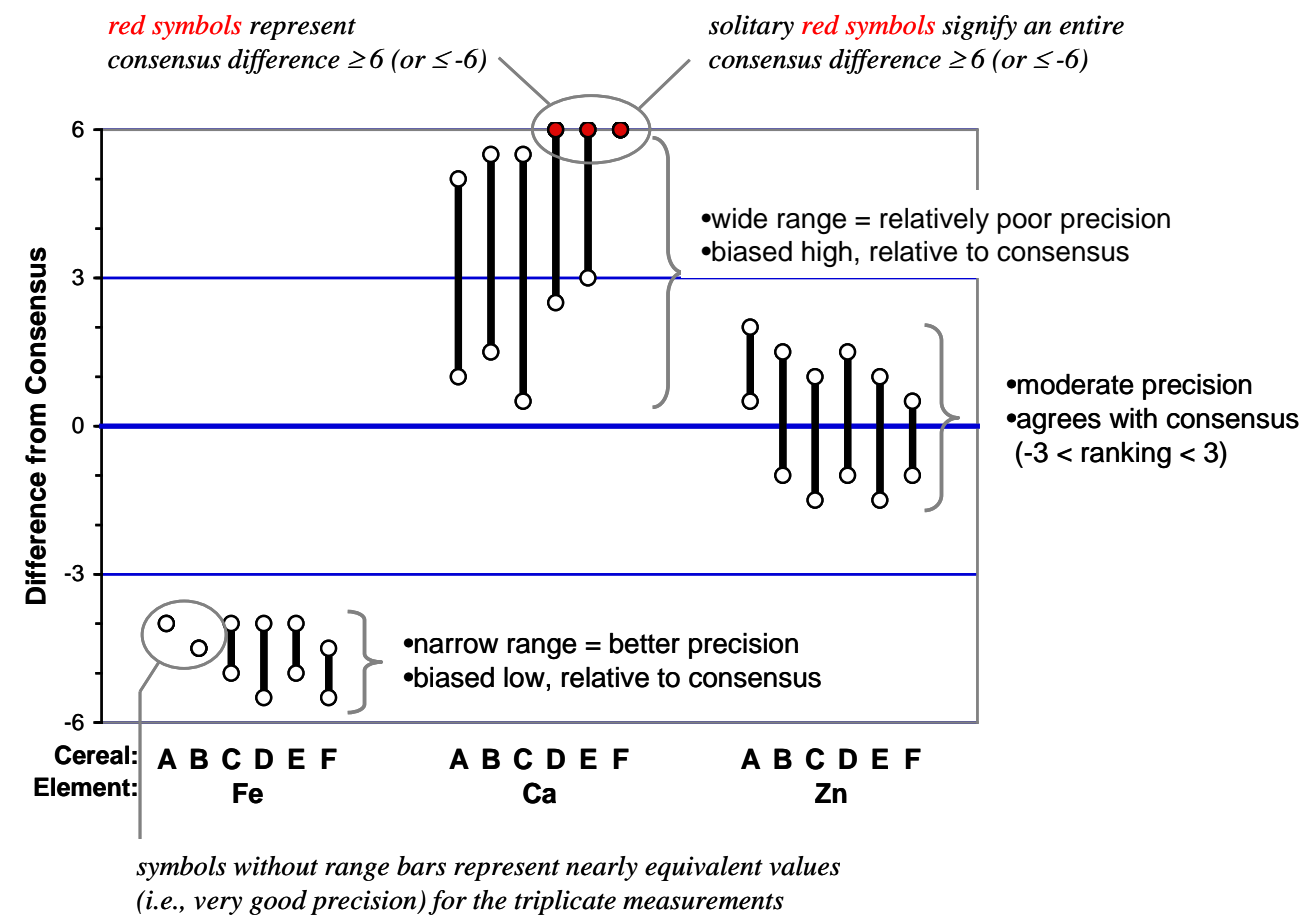




\section{Part II. Your Individual Laboratory Results (Lab Code: E509)}

Your laboratory's data is summarized in Table Z $\mathbf{Z}$. The mean (average) \pm 1 standard deviation were determined from the reported triplicate $(n=3)$ values for each of the 18 measurements $(\mathrm{Fe}, \mathrm{Ca}$ and Zn within the six study materials, Cereals A-F) in Exercise E.

Your laboratory's summary consensus plot for Fe, Ca and $\mathrm{Zn}$ in the six cereal study materials is provided in Figure $\mathbf{Z}$. The data have been normalized relative to consensus means and Horn-HornDuncan (HHD) uncertainties so that the consensus value is equal to zero. The thick blue line in this plot represents the consensus values for each of the 18 measurements (Fe, $\mathrm{Ca}$ and $\mathrm{Zn}$ within the six study materials, Cereals A-F). The thin blue lines represent \pm 3 HHD standard uncertainty intervals about the consensus values. The figure is scaled so that the top and bottom of the Y-axis represent \pm 6 HHD intervals. Your laboratory's results for each of the 18 measurements are illustrated by the black bars. The ends of bars that are entirely within the \pm 6 HHD intervals are marked with an open circle (०); red circles $(\bullet)$ indicate that some or all of the bar is outside the \pm 6 HHD interval.

The length of each bar indicates how well your measurements agree with themselves. The distance from the center of the bars to the consensus line indicates how well your measurements agree with those of the other participants in this study. (An example consensus plot with explanations is also included to aid in the interpretation your results.) As a general rule, we consider results that fall within \pm 3 HHD standard uncertainty lines to be within consensus (approximate 99\% confidence limit) and results that have a black bar length $\approx 3$ HHD units are considered as acceptable precision. If values are consistently very high or low for a particular element, then a calibration problem may exist. In such cases, it is important to make sure your calibration standards are from a reliable source, the quality is confirmed and that your calibration solutions are correctly prepared and stored. In addition, calibration points must be linear in the expected measurement range and closely surround expected values.

The means of your laboratory's measurement results for $\mathrm{Ca}$ and $\mathrm{Zn}$ in the rice ground and flake cereal pairs ( $D \& E$ ) and Fe in the wheat cereals (B \& C) agree well with each other and with the consensus means, indicating a consistent sample preparation process. The values for $\mathrm{Zn}$ in $\mathrm{B}$ \& $\mathrm{C}$ do not agree well; the relatively high value for $\mathrm{Zn}$ in wheat flake cereal $\mathrm{C}$ may result from contamination of cereal $\mathrm{C}$, either during packaging or during sample processing. This could also be due to a calculation error which was applied to all three values for $\mathrm{Zn}$ in cereal C. The precision of your measurement results is fairly variable, ranging from quite good (bars of 1 to 2 HHD units in length) to marginal (bars longer than 3 HHD units). In combination with the generally low results relative to consensus, this may suggest that your sample digestion process is somewhat inconsistent.

Table Z. Summary of Fe, Ca and Zn values within the six study materials, Cereals A-F

\begin{tabular}{|c|cccccc|}
\hline Lab Code: E509 & \multicolumn{5}{|c|}{ Mean Results $\pm \mathbf{1}$ SD (n= 3) } \\
\hline & $\begin{array}{c}\text { Cereal A } \\
\text { Wheat } \\
\text { Ground } \\
\text { (SRM 3233) }\end{array}$ & $\begin{array}{c}\text { Cereal B } \\
\text { Wheat } \\
\text { Ground }\end{array}$ & $\begin{array}{c}\text { Cereal C } \\
\text { Wheat } \\
\text { Flake }\end{array}$ & $\begin{array}{c}\text { Cereal D } \\
\text { Rice } \\
\text { Ground }\end{array}$ & $\begin{array}{c}\text { Cereal E } \\
\text { Rice } \\
\text { Flake }\end{array}$ & $\begin{array}{c}\text { Cereal F } \\
\text { Wheat/Rice } \\
\text { Crushed }\end{array}$ \\
\hline $\mathrm{Fe}(\mu \mathrm{g} / \mathrm{g})$ & $720 \pm 26$ & $787 \pm 25$ & $820 \pm 20$ & $453 \pm 6$ & $427 \pm 31$ & $603 \pm 15$ \\
$\mathrm{Ca}(\mu \mathrm{g} / \mathrm{g})$ & $35100 \pm 850$ & $34000 \pm 0$ & $33800 \pm 720$ & $197 \pm 12$ & $207 \pm 6$ & $16700 \pm 1210$ \\
$\mathrm{Zn}(\mu \mathrm{g} / \mathrm{g})$ & $547 \pm 12$ & $610 \pm 10$ & $820 \pm 20$ & $19 \pm 1$ & $19 \pm 1$ & $313 \pm 15$ \\
\hline
\end{tabular}


Figure Z. A consensus plot summarizing your individual results (Lab Code: E509)

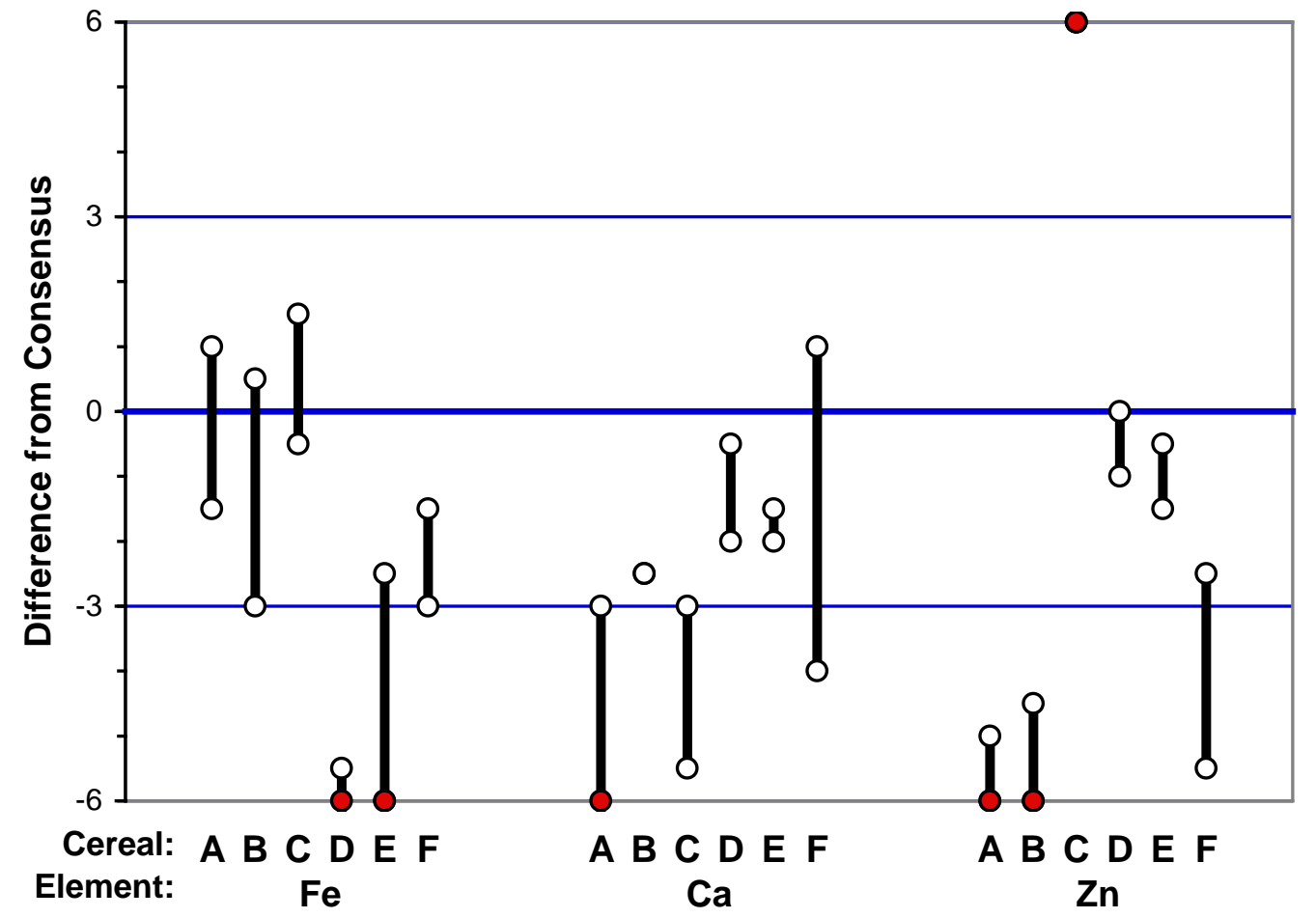

How to interpret your consensus summary plot:

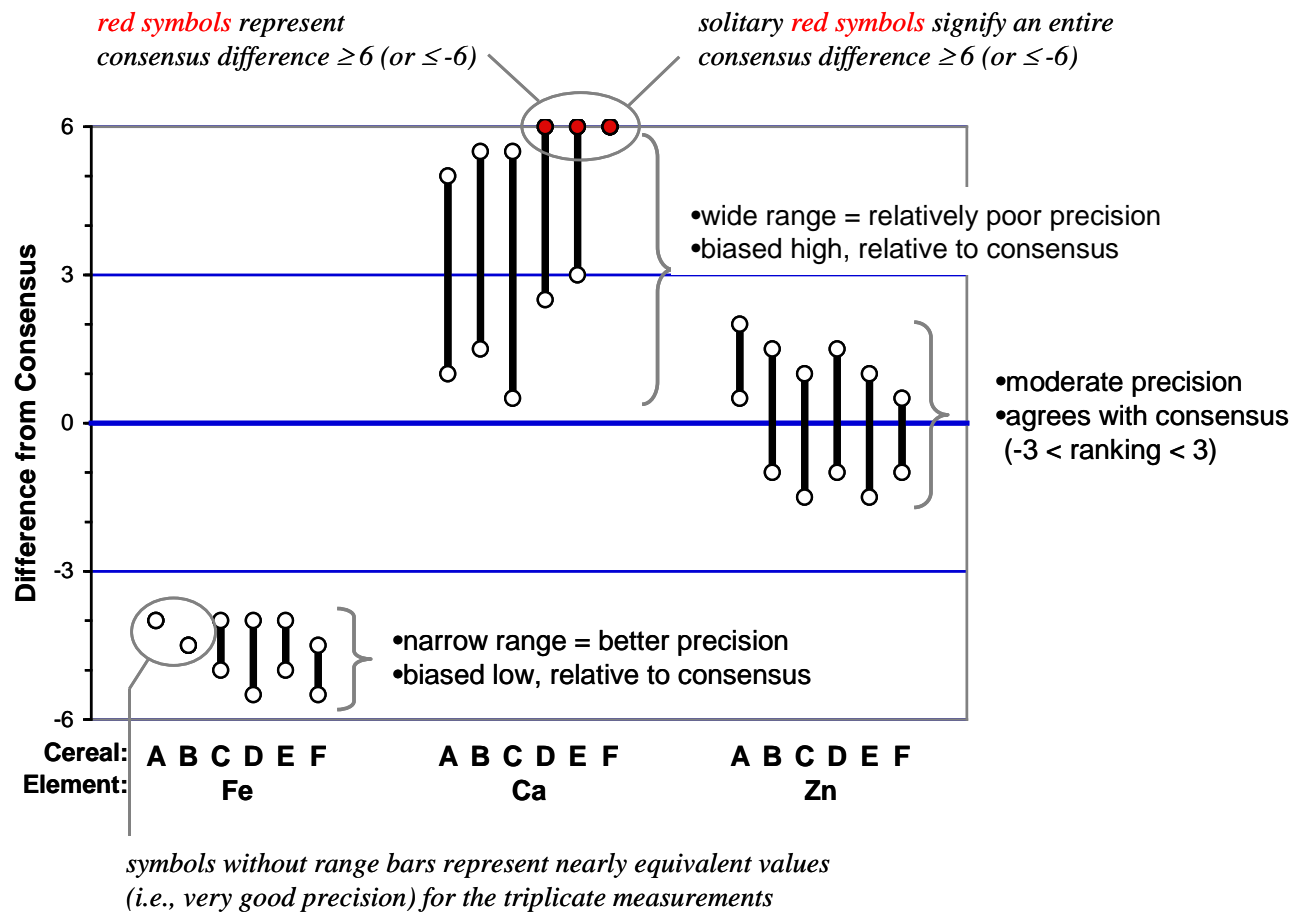




\section{Part II. Your Individual Laboratory Results (Lab Code: E510)}

Your laboratory's data is summarized in Table Z. The mean (average) \pm 1 standard deviation were determined from the reported triplicate $(\mathrm{n}=3$ ) values for each of the 18 measurements ( $\mathrm{Fe}, \mathrm{Ca}$ and $\mathrm{Zn}$ within the six study materials, Cereals A-F) in Exercise E.

Your laboratory's summary consensus plot for $\mathrm{Fe}, \mathrm{Ca}$ and $\mathrm{Zn}$ in the six cereal study materials is provided in Figure Z . The data have been normalized relative to consensus means and Horn-HornDuncan (HHD) uncertainties so that the consensus value is equal to zero. The thick blue line in this plot represents the consensus values for each of the 18 measurements ( $\mathrm{Fe}, \mathrm{Ca}$ and $\mathrm{Zn}$ within the six study materials, Cereals A-F). The thin blue lines represent \pm 3 HHD standard uncertainty intervals about the consensus values. The figure is scaled so that the top and bottom of the Y-axis represent \pm 6 HHD intervals. Your laboratory's results for each of the 18 measurements are illustrated by the black bars. The ends of bars that are entirely within the \pm 6 HHD intervals are marked with an open circle (०); red circles ( $\bullet$ ) indicate that some or all of the bar is outside the $\pm 6 \mathrm{HHD}$ interval.

The length of each bar indicates how well your measurements agree with themselves. The distance from the center of the bars to the consensus line indicates how well your measurements agree with those of the other participants in this study. (An example consensus plot with explanations is also included to aid in the interpretation your results.) As a general rule, we consider results that fall within \pm 3 HHD standard uncertainty lines to be within consensus (approximate $99 \%$ confidence limit) and results that have a black bar length $\approx 3$ HHD units are considered as acceptable precision. If values are consistently very high or low for a particular element, then a calibration problem may exist. In such cases, it is important to make sure your calibration standards are from a reliable source, the quality is confirmed and that your calibration solutions are correctly prepared and stored. In addition, calibration points must be linear in the expected measurement range and closely surround expected values.

Your laboratory's measurement results are pretty consistently below the consensus means; many values are below the $6 \mathrm{HHD}$ limits of the graph. These very low values suggest that your samples were incompletely digested. Your laboratory was the only participant that took sample replicates from both sets of bottles or packages. This may contribute to the relatively large variability of all elements in Cereals $\mathrm{C}$ and $\mathrm{E}$ as homogeneity within the package may be an issue with these flake materials.

Table Z. Summary of Fe, Ca and Zn values within the six study materials, Cereals A-F

\begin{tabular}{|c|cccccc|}
\hline Lab Code: E510 & \multicolumn{7}{|c|}{ Mean Results \pm 1 SD $(\mathbf{n}=\mathbf{3})$} \\
\hline & $\begin{array}{c}\text { Cereal A } \\
\text { Wheat } \\
\text { Ground } \\
(\text { SRM 3233) }\end{array}$ & $\begin{array}{c}\text { Cereal B } \\
\text { Wheat } \\
\text { Ground }\end{array}$ & $\begin{array}{c}\text { Cereal C } \\
\text { Wheat } \\
\text { Flake }\end{array}$ & $\begin{array}{c}\text { Cereal D } \\
\text { Rice } \\
\text { Ground }\end{array}$ & $\begin{array}{c}\text { Cereal E } \\
\text { Rice } \\
\text { Flake }\end{array}$ & $\begin{array}{c}\text { Cereal F } \\
\text { Wheat//Rice } \\
\text { Crushed }\end{array}$ \\
\hline $\mathrm{Fe}(\mu \mathrm{g} / \mathrm{g})$ & $683 \pm 9$ & $732 \pm 14$ & $667 \pm 96$ & $460 \pm 25$ & $384 \pm 33$ & $500 \pm 25$ \\
$\mathrm{Ca}(\mu \mathrm{g} / \mathrm{g})$ & $32850 \pm 650$ & $32010 \pm 330$ & $30740 \pm 790$ & $195 \pm 7$ & $233 \pm 43$ & $16300 \pm 350$ \\
$\mathrm{Zn}(\mu \mathrm{g} / \mathrm{g})$ & $536 \pm 30$ & $587 \pm 38$ & $522 \pm 66$ & $20 \pm 1$ & $18 \pm 2$ & $284 \pm 7$ \\
\hline
\end{tabular}


Figure Z. A consensus plot summarizing your individual results (Lab Code: E510)

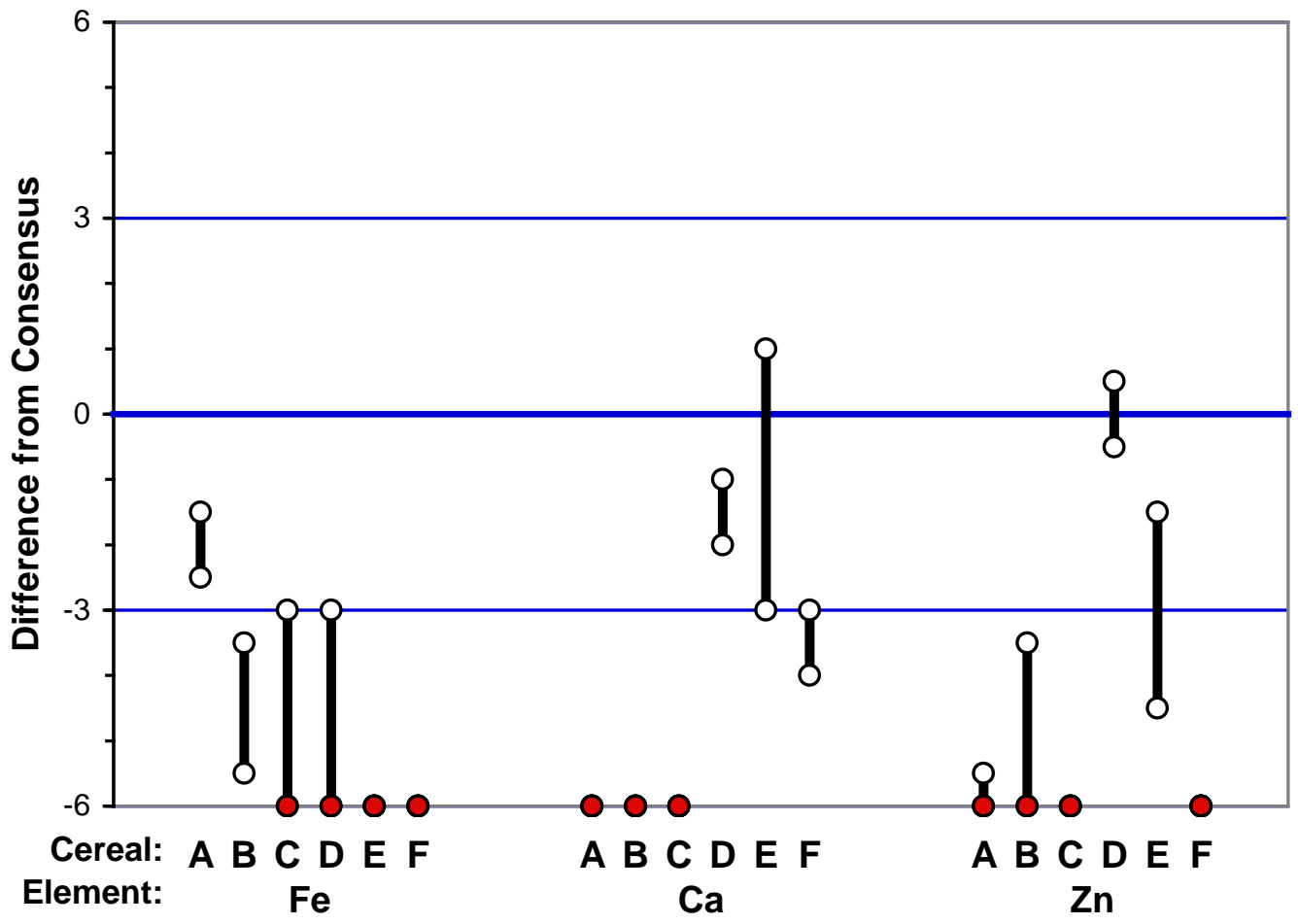

How to interpret your consensus summary plot:

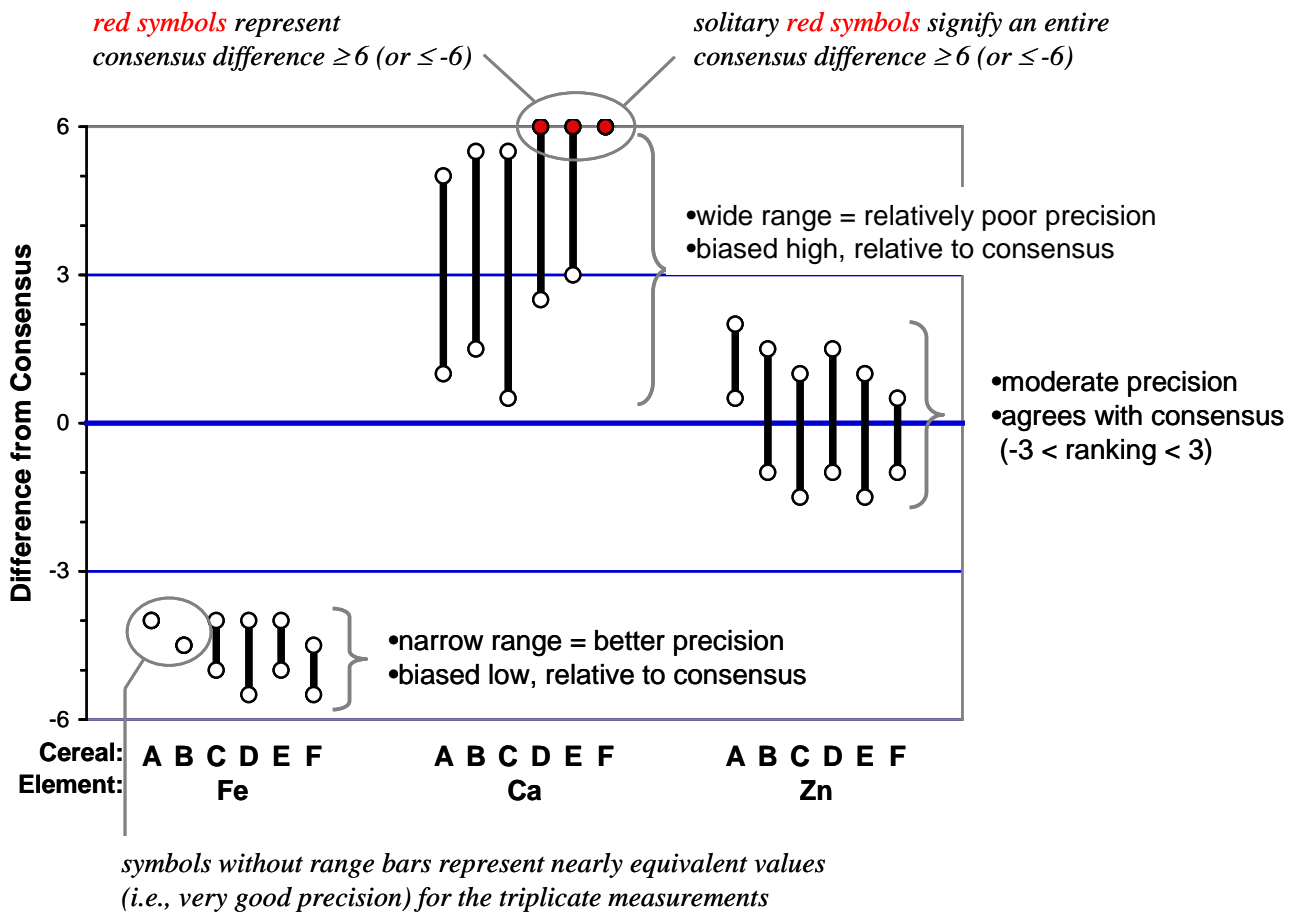




\section{Part II. Your Individual Laboratory Results (Lab Code: E511)}

Your laboratory's data is summarized in Table Z. The mean (average) \pm 1 standard deviation were determined from the reported triplicate $(n=3)$ values for each of the 18 measurements $(\mathrm{Fe}$, $\mathrm{Ca}$ and $\mathrm{Zn}$ within the six study materials, Cereals A-F) in Exercise E.

Your laboratory's summary consensus plot for $\mathrm{Fe}, \mathrm{Ca}$ and $\mathrm{Zn}$ in the six cereal study materials is provided in Figure Z. The data have been normalized relative to consensus means and Horn-HornDuncan (HHD) uncertainties so that the consensus value is equal to zero. The thick blue line in this plot represents the consensus values for each of the 18 measurements (Fe, $\mathrm{Ca}$ and $\mathrm{Zn}$ within the six study materials, Cereals A-F). The thin blue lines represent \pm 3 HHD standard uncertainty intervals about the consensus values. The figure is scaled so that the top and bottom of the Y-axis represent \pm 6 HHD intervals. Your laboratory's results for each of the 18 measurements are illustrated by the black bars. The ends of bars that are entirely within the \pm 6 HHD intervals are marked with an open circle (०); red circles $(\bullet)$ indicate that some or all of the bar is outside the \pm 6 HHD interval.

The length of each bar indicates how well your measurements agree with themselves. The distance from the center of the bars to the consensus line indicates how well your measurements agree with those of the other participants in this study. (An example consensus plot with explanations is also included to aid in the interpretation your results.) As a general rule, we consider results that fall within \pm 3 HHD standard uncertainty lines to be within consensus (approximate 99\% confidence limit) and results that have a black bar length $\approx 3$ HHD units are considered as acceptable precision. If values are consistently very high or low for a particular element, then a calibration problem may exist. In such cases, it is important to make sure your calibration standards are from a reliable source, the quality is confirmed and that your calibration solutions are correctly prepared and stored. In addition, calibration points must be linear in the expected measurement range and closely surround expected values.

Your laboratory's measurement results generally agree well with the consensus means indicating good calibration. The mean values for Fe, $\mathrm{Ca}$ and $\mathrm{Zn}$ in the wheat ground and flake cereal pairs (B \& C) and the rice ground and flake cereal pairs (D \& E) pairs agree quite well, indicating a quite consistent sample preparation process. Your laboratory's values for the three elements in all materials are mostly moderately precise (many bar lengths of 2 to 3 HHD units). The somewhat less precise (slightly longer bars) values in Cereal A relative to the other materials is counter to our expectation, given the established homogeneity of Cereal A.

Table Z. Summary of Fe, Ca and Zn values within the six study materials, Cereals A-F

\begin{tabular}{|c|cccccc|}
\hline Lab Code: E511 & \multicolumn{5}{|c|}{ Mean Results $\pm \mathbf{1}$ SD (n= 3) } \\
\hline & $\begin{array}{c}\text { Cereal A } \\
\text { Wheat } \\
\text { Ground } \\
\text { (SRM 3233) }\end{array}$ & $\begin{array}{c}\text { Cereal B } \\
\text { Wheat } \\
\text { Ground }\end{array}$ & $\begin{array}{c}\text { Cereal C } \\
\text { Wheat } \\
\text { Flake }\end{array}$ & $\begin{array}{c}\text { Cereal D } \\
\text { Rice } \\
\text { Ground }\end{array}$ & $\begin{array}{c}\text { Cereal E } \\
\text { Rice } \\
\text { Flake }\end{array}$ & $\begin{array}{c}\text { Cereal F } \\
\text { Wheat/Rice } \\
\text { Crushed }\end{array}$ \\
\hline Fe $(\mu \mathrm{g} / \mathrm{g})$ & $747 \pm 25$ & $806 \pm 23$ & $818 \pm 29$ & $516 \pm 12$ & $506 \pm 8$ & $654 \pm 22$ \\
$\mathrm{Ca}(\mu \mathrm{g} / \mathrm{g})$ & $36630 \pm 1120$ & $34430 \pm 920$ & $34830 \pm 1010$ & $196 \pm 6$ & $199 \pm 8$ & $17700 \pm 600$ \\
$\mathrm{Zn}(\mu \mathrm{g} / \mathrm{g})$ & $609 \pm 19$ & $675 \pm 17$ & $688 \pm 22$ & $20 \pm 1$ & $20 \pm 1$ & $357 \pm 5$ \\
\hline
\end{tabular}


Figure Z. A consensus plot summarizing your individual results (Lab Code: E511)

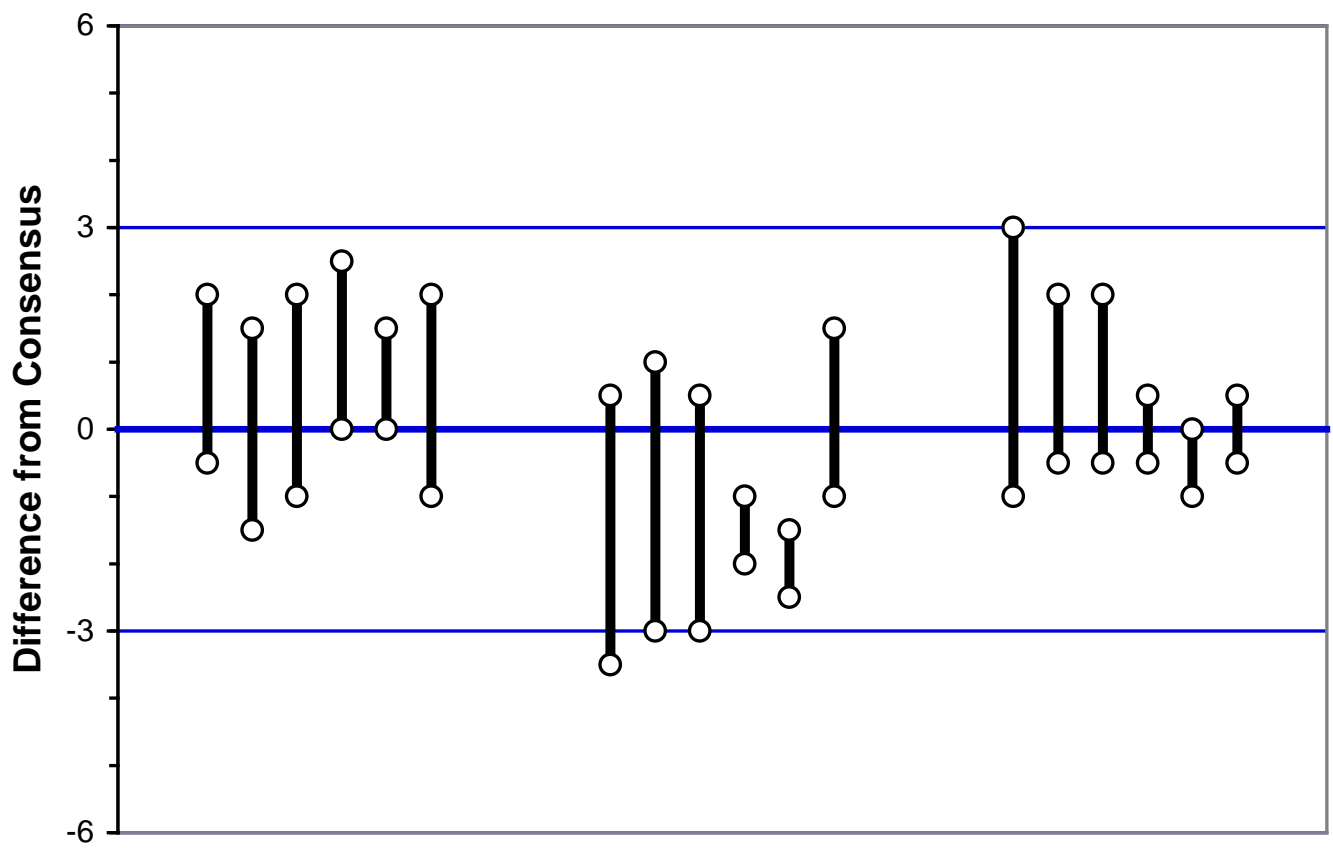
Cereal: A B C D E F
Element:
$\mathrm{Fe}$
$\underset{C a}{A}$ B $D E$
A B C D E F
$\mathrm{Zn}$

How to interpret your consensus summary plot:

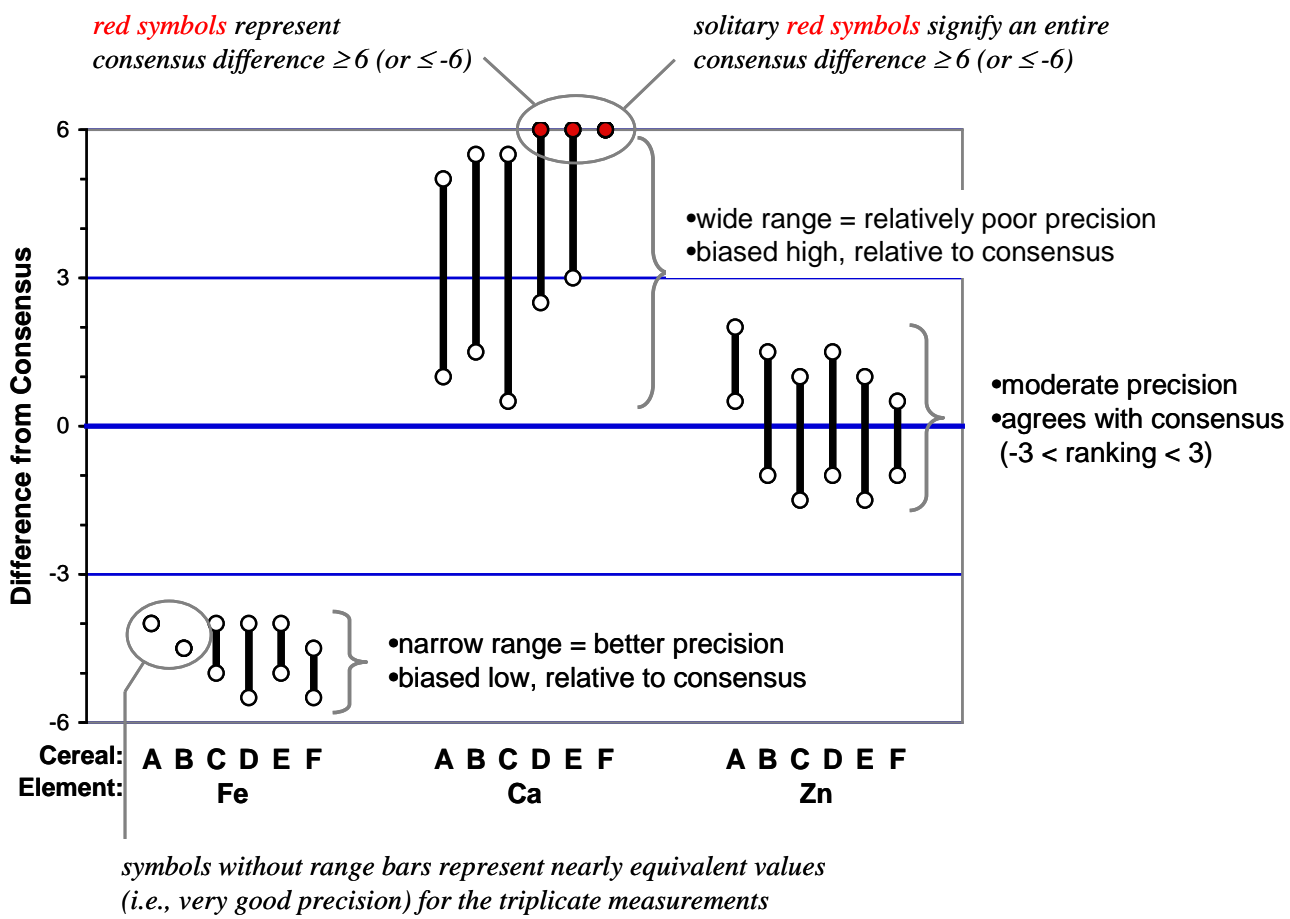




\section{Part II. Your Individual Laboratory Results (Lab Code: E513)}

Your laboratory's data is summarized in Table Z. The mean (average) \pm 1 standard deviation were determined from the reported triplicate $(n=3)$ values for each of the 18 measurements (Fe, $\mathrm{Ca}$ and $\mathrm{Zn}$ within the six study materials, Cereals A-F) in Exercise E.

Your laboratory's summary consensus plot for Fe, Ca and $\mathrm{Zn}$ in the six cereal study materials is provided in Figure Z $\mathbf{Z}$. The data have been normalized relative to consensus means and Horn-HornDuncan (HHD) uncertainties so that the consensus value is equal to zero. The thick blue line in this plot represents the consensus values for each of the 18 measurements (Fe, $\mathrm{Ca}$ and $\mathrm{Zn}$ within the six study materials, Cereals A-F). The thin blue lines represent \pm 3 HHD standard uncertainty intervals about the consensus values. The figure is scaled so that the top and bottom of the Y-axis represent \pm 6 HHD intervals. Your laboratory's results for each of the 18 measurements are illustrated by the black bars. The ends of bars that are entirely within the \pm 6 HHD intervals are marked with an open circle (०); red circles $(\bullet)$ indicate that some or all of the bar is outside the \pm 6 HHD interval.

The length of each bar indicates how well your measurements agree with themselves. The distance from the center of the bars to the consensus line indicates how well your measurements agree with those of the other participants in this study. (An example consensus plot with explanations is also included to aid in the interpretation your results.) As a general rule, we consider results that fall within \pm 3 HHD standard uncertainty lines to be within consensus (approximate 99\% confidence limit) and results that have a black bar length $\approx 3$ HHD units are considered as acceptable precision. If values are consistently very high or low for a particular element, then a calibration problem may exist. In such cases, it is important to make sure your calibration standards are from a reliable source, the quality is confirmed and that your calibration solutions are correctly prepared and stored. In addition, calibration points must be linear in the expected measurement range and closely surround expected values.

Your laboratory's measurement results for Fe mostly agree well with the consensus results. Many of your results for $\mathrm{Ca}$ and $\mathrm{Zn}$ are lower than consensus; you may want to check your Ca and Zn calibration standards. The mean values for all three elements in the ground and flake rice cereal pairs (D \& E) agree well, as do those for Ca and Zn in the ground and flake wheat cereal pairs (B \& C); this suggests that your sample preparation process was fairly consistent. Your results are mostly quite precise (bar lengths of less than 2 HHD units), except for Ca in the wheat-based cereals (A, B \& $\mathrm{C})$. This relatively poor precision in these high Ca materials suggests that the mass fraction of $\mathrm{Ca}$ in the samples analyzed may have been above the linear region of your calibration curve. It is important when preparing samples to have appropriate concentration levels and to use enough calibration points to adequately cover the expected concentration range to ensure linearity.

Table Z. Summary of Fe, Ca and Zn values within the six study materials, Cereals A-F

\begin{tabular}{|c|cccccc|}
\hline Lab Code: E513 & \multicolumn{5}{|c|}{ Mean Results $\pm \mathbf{1}$ SD (n= 3) } \\
\hline & $\begin{array}{c}\text { Cereal A } \\
\text { Wheat } \\
\text { Ground } \\
\text { (SRM 3233) }\end{array}$ & $\begin{array}{c}\text { Cereal B } \\
\text { Wheat } \\
\text { Ground }\end{array}$ & $\begin{array}{c}\text { Cereal C } \\
\text { Wheat } \\
\text { Flake }\end{array}$ & $\begin{array}{c}\text { Cereal D } \\
\text { Rice } \\
\text { Ground }\end{array}$ & $\begin{array}{c}\text { Cereal E } \\
\text { Rice } \\
\text { Flake }\end{array}$ & $\begin{array}{c}\text { Cereal F } \\
\text { Wheat/Rice } \\
\text { Crushed }\end{array}$ \\
\hline $\mathrm{Fe}(\mu \mathrm{g} / \mathrm{g})$ & $743 \pm 6$ & $773 \pm 6$ & $800 \pm 10$ & $513 \pm 12$ & $500 \pm 0$ & $660 \pm 10$ \\
$\mathrm{Ca}(\mu \mathrm{g} / \mathrm{g})$ & $37000 \pm 1000$ & $34000 \pm 1000$ & $34670 \pm 1,160$ & $180 \pm 0$ & $180 \pm 0$ & $16670 \pm 580$ \\
$\mathrm{Zn}(\mu \mathrm{g} / \mathrm{g})$ & $533 \pm 6$ & $573 \pm 6$ & $587 \pm 6$ & $18 \pm 1$ & $18 \pm 1$ & $320 \pm 17$ \\
\hline
\end{tabular}


Figure Z. A consensus plot summarizing your individual results (Lab Code: E513)

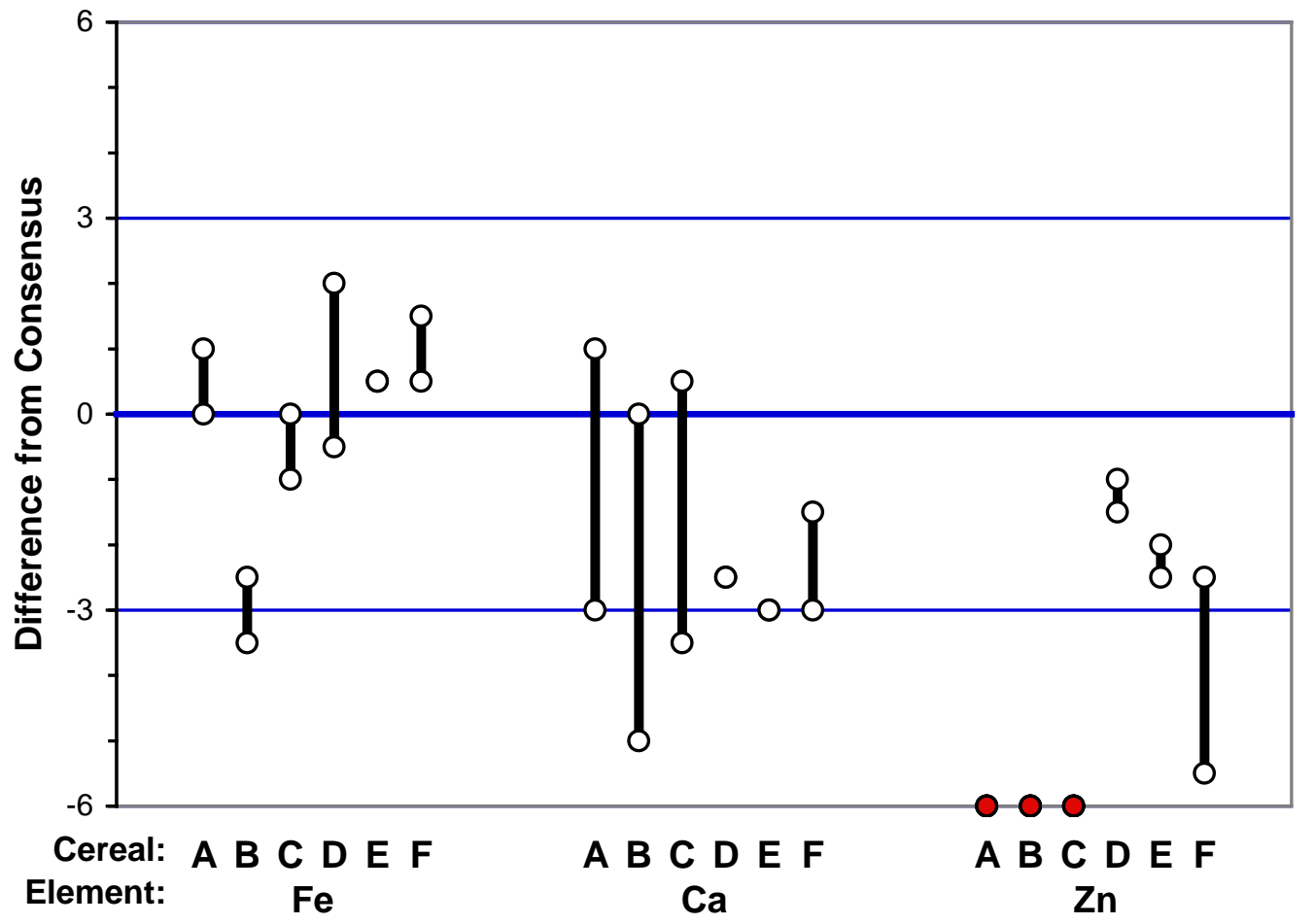

How to interpret your consensus summary plot:

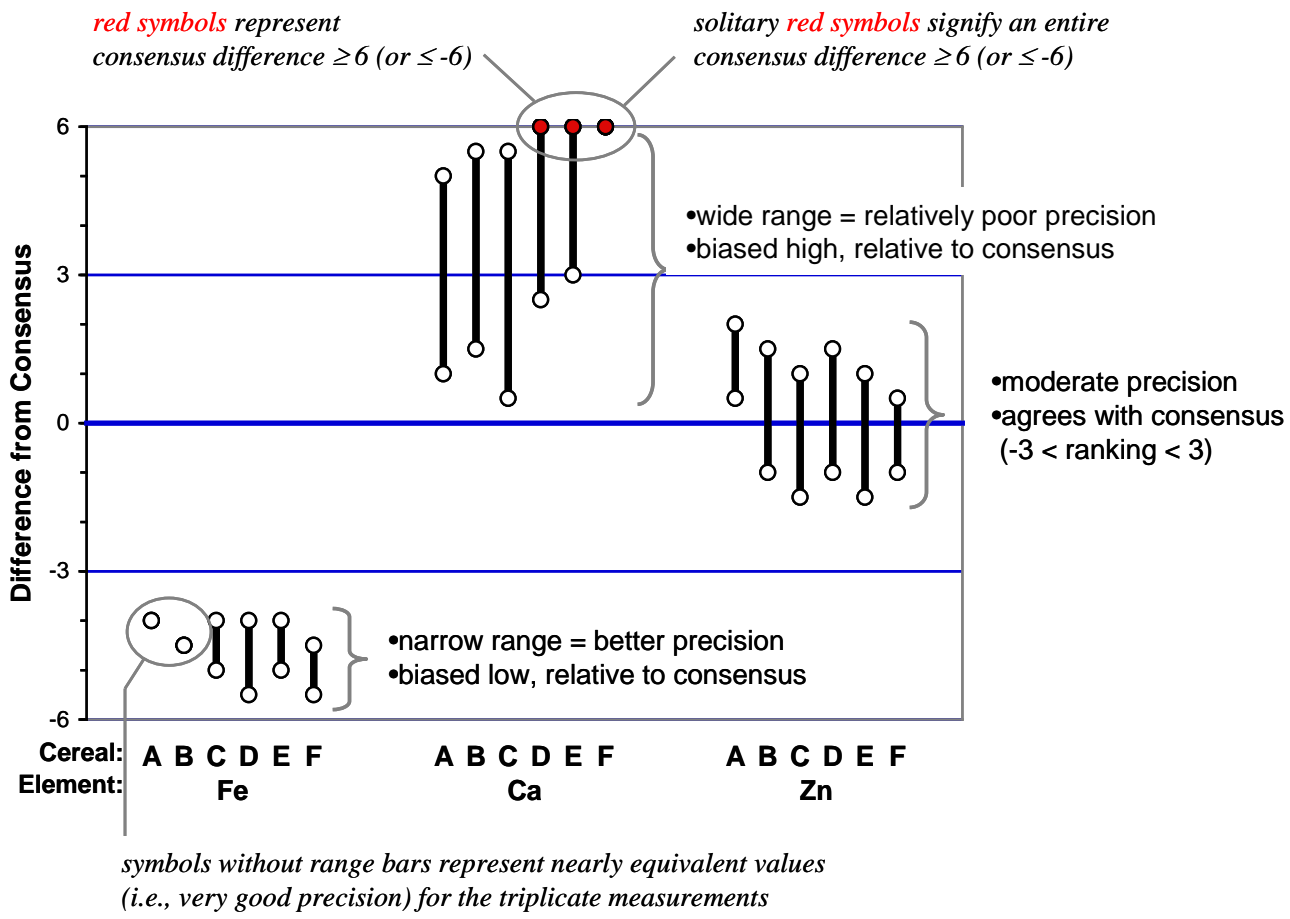




\section{Part II. Your Individual Laboratory Results (Lab Code: E515)}

Your laboratory's data is summarized in Table Z. The mean (average) \pm 1 standard deviation were determined from the reported triplicate $(n=3)$ values for each of the 18 measurements $(\mathrm{Fe}, \mathrm{Ca}$ and Zn within the six study materials, Cereals A-F) in Exercise E.

Your laboratory's summary consensus plot for $\mathrm{Fe}, \mathrm{Ca}$ and $\mathrm{Zn}$ in the six cereal study materials is provided in Figure Z. The data have been normalized relative to consensus means and Horn-HornDuncan (HHD) uncertainties so that the consensus value is equal to zero. The thick blue line in this plot represents the consensus values for each of the 18 measurements (Fe, $\mathrm{Ca}$ and $\mathrm{Zn}$ within the six study materials, Cereals A-F). The thin blue lines represent \pm 3 HHD standard uncertainty intervals about the consensus values. The figure is scaled so that the top and bottom of the Y-axis represent \pm 6 HHD intervals. Your laboratory's results for each of the 18 measurements are illustrated by the black bars. The ends of bars that are entirely within the \pm 6 HHD intervals are marked with an open circle (०); red circles $(\bullet)$ indicate that some or all of the bar is outside the \pm 6 HHD interval.

The length of each bar indicates how well your measurements agree with themselves. The distance from the center of the bars to the consensus line indicates how well your measurements agree with those of the other participants in this study. (An example consensus plot with explanations is also included to aid in the interpretation your results.) As a general rule, we consider results that fall within \pm 3 HHD standard uncertainty lines to be within consensus (approximate 99\% confidence limit) and results that have a black bar length $\approx 3$ HHD units are considered as acceptable precision. If values are consistently very high or low for a particular element, then a calibration problem may exist. In such cases, it is important to make sure your calibration standards are from a reliable source, the quality is confirmed and that your calibration solutions are correctly prepared and stored. In addition, calibration points must be linear in the expected measurement range and closely surround expected values.

Your laboratory's measurement results for the ground and flake wheat cereal pairs (B \& C) and ground and flake rice cereal pairs (D \& E) agree fairly well, indicating that your sample preparation process is fairly consistent. However, many of your laboratory's measurement results are inconsistently above or below the consensus means. The precision of your measurements likewise is rather inconsistent, ranging from quite good (bars less that $1 \mathrm{HHD}$ unit long) to moderately poor (bars longer than 3 HHD units). These results suggest inconsistent sample digestion and/or sample dilution.

Table Z. Summary of Fe, Ca and Zn values within the six study materials, Cereals A-F

\begin{tabular}{|c|cccccc|}
\hline Lab Code: E515 & \multicolumn{5}{|c|}{ Mean Results $\pm \mathbf{1}$ SD (n= 3) } \\
\hline & $\begin{array}{c}\text { Cereal A } \\
\text { Wheat } \\
\text { Ground } \\
\text { (SRM 3233) }\end{array}$ & $\begin{array}{c}\text { Cereal B } \\
\text { Wheat } \\
\text { Ground }\end{array}$ & $\begin{array}{c}\text { Cereal C } \\
\text { Wheat } \\
\text { Flake }\end{array}$ & $\begin{array}{c}\text { Cereal D } \\
\text { Rice } \\
\text { Ground }\end{array}$ & $\begin{array}{c}\text { Cereal E } \\
\text { Rice } \\
\text { Flake }\end{array}$ & $\begin{array}{c}\text { Cereal F } \\
\text { Wheat/Rice } \\
\text { Crushed }\end{array}$ \\
\hline $\mathrm{Fe}(\mu \mathrm{g} / \mathrm{g})$ & $805 \pm 14$ & $899 \pm 12$ & $908 \pm 15$ & $472 \pm 13$ & $487 \pm 35$ & $623 \pm 10$ \\
$\mathrm{Ca}(\mu \mathrm{g} / \mathrm{g})$ & $35320 \pm 310$ & $33270 \pm 390$ & $34160 \pm 700$ & $233 \pm 2$ & $238 \pm 10$ & $13130 \pm 1100$ \\
$\mathrm{Zn}(\mu \mathrm{g} / \mathrm{g})$ & $648 \pm 3$ & $723 \pm 21$ & $756 \pm 13$ & $26 \pm 0$ & $25 \pm 1$ & $299 \pm 26$ \\
\hline
\end{tabular}


Figure Z. A consensus plot summarizing your individual results (Lab Code: E515)

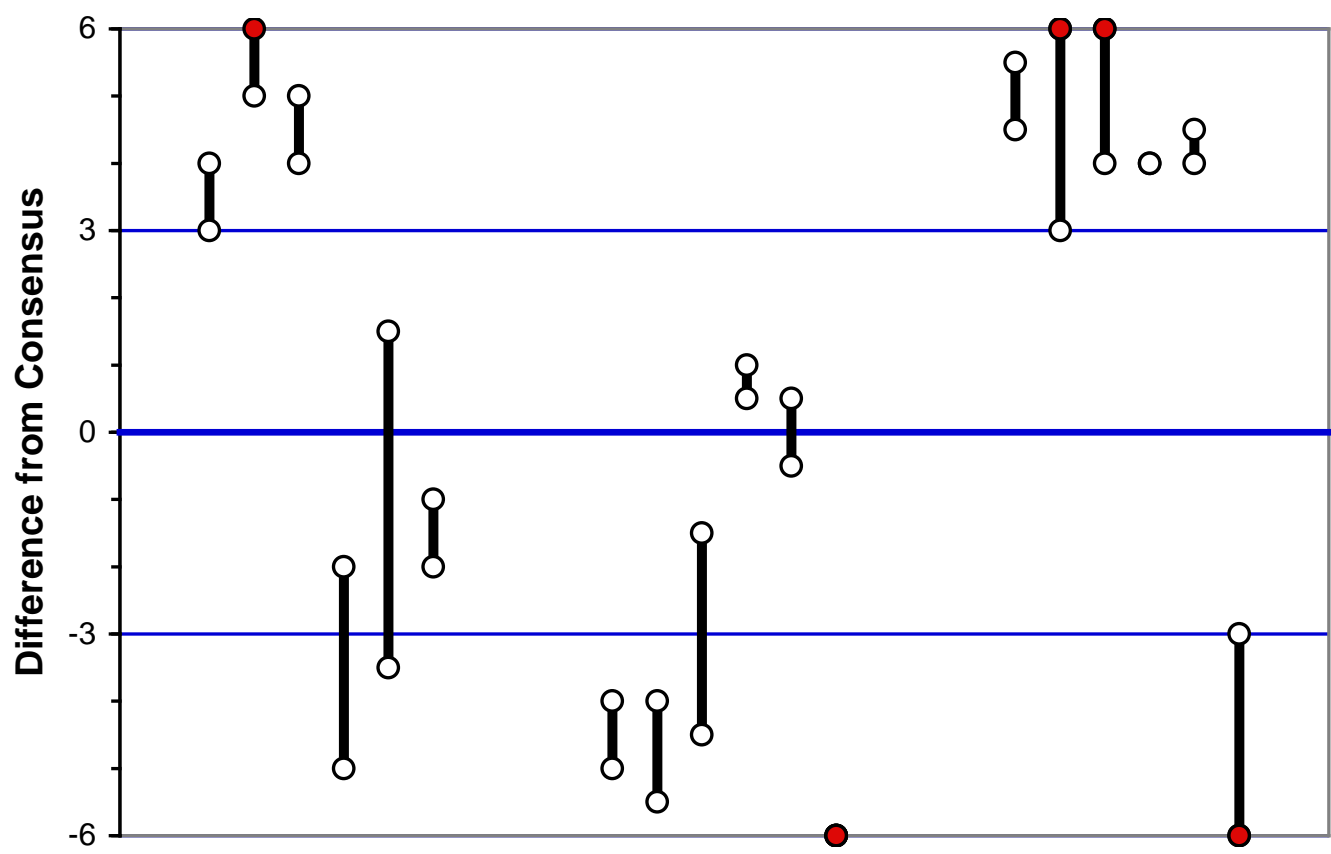
Cereal: A B C D E F
Element:
$\mathrm{Fe}$
A B C D E F
A B C D E F
$\mathrm{Ca}$
$\mathrm{Zn}$

How to interpret your consensus summary plot:

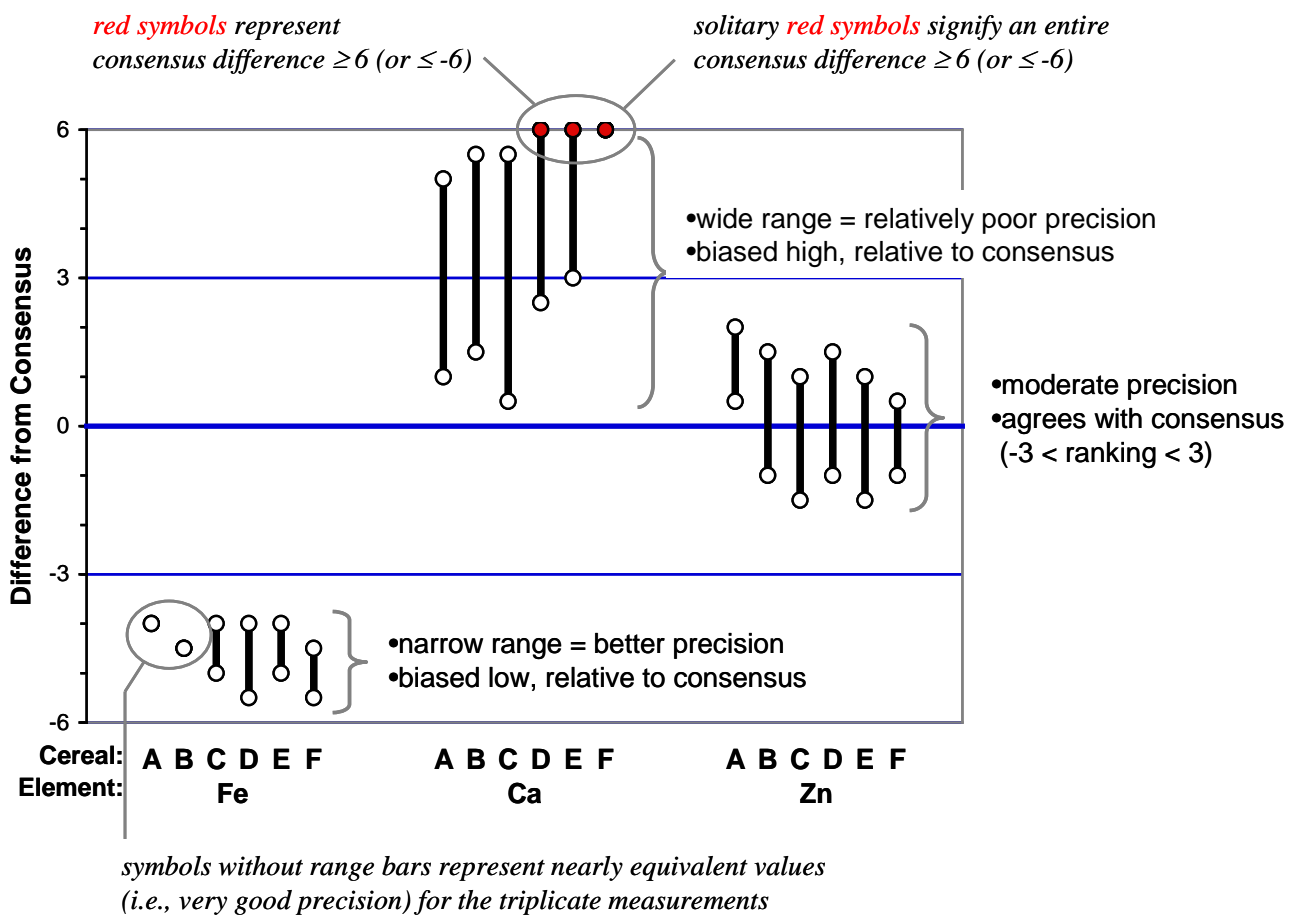




\section{Part II. Your Individual Laboratory Results (Lab Code: E520)}

Your laboratory's data is summarized in Table Z. The mean (average) \pm 1 standard deviation were determined from the reported triplicate $(n=3)$ values for each of the 18 measurements $(\mathrm{Fe}, \mathrm{Ca}$ and Zn within the six study materials, Cereals A-F) in Exercise E.

Your laboratory's summary consensus plot for Fe, Ca and $\mathrm{Zn}$ in the six cereal study materials is provided in Figure Z $\mathbf{Z}$. The data have been normalized relative to consensus means and Horn-HornDuncan (HHD) uncertainties so that the consensus value is equal to zero. The thick blue line in this plot represents the consensus values for each of the 18 measurements (Fe, $\mathrm{Ca}$ and $\mathrm{Zn}$ within the six study materials, Cereals A-F). The thin blue lines represent \pm 3 HHD standard uncertainty intervals about the consensus values. The figure is scaled so that the top and bottom of the Y-axis represent \pm 6 HHD intervals. Your laboratory's results for each of the 18 measurements are illustrated by the black bars. The ends of bars that are entirely within the \pm 6 HHD intervals are marked with an open circle (०); red circles $(\bullet)$ indicate that some or all of the bar is outside the \pm 6 HHD interval.

The length of each bar indicates how well your measurements agree with themselves. The distance from the center of the bars to the consensus line indicates how well your measurements agree with those of the other participants in this study. (An example consensus plot with explanations is also included to aid in the interpretation your results.) As a general rule, we consider results that fall within \pm 3 HHD standard uncertainty lines to be within consensus (approximate 99\% confidence limit) and results that have a black bar length $\approx 3$ HHD units are considered as acceptable precision. If values are consistently very high or low for a particular element, then a calibration problem may exist. In such cases, it is important to make sure your calibration standards are from a reliable source, the quality is confirmed and that your calibration solutions are correctly prepared and stored. In addition, calibration points must be linear in the expected measurement range and closely surround expected values.

Your laboratory's measurement results for all three elements in the rice cereal (D \& E) pair agree well with each other, indicating a consistent sample preparation process for this material. Your results are in general quite precise (bar lengths of 1 to 2 HHD units) for all elements and all materials. However, with the exception of $\mathrm{Ca}$ and $\mathrm{Zn}$ in the rice-based cereals (D, E and F), your measurement values are consistently higher than the consensus values. Given that $\mathrm{Ca}$ and $\mathrm{Zn}$ require very different dilution schemes for the wheat-based and rice-based materials, this unusual pattern suggests problems with calibration and/or sample preparation steps. It is important to prepare samples that will have appropriate concentration levels and to use calibration points to adequately cover the expected concentration range.

Table Z. Summary of Fe, Ca and Zn values within the six study materials, Cereals A-F

\begin{tabular}{|c|cccccc|}
\hline Lab Code: E520 & \multicolumn{5}{|c|}{ Mean Results $\pm \mathbf{1}$ SD (n= 3) } \\
\hline & $\begin{array}{c}\text { Cereal A } \\
\text { Wheat } \\
\text { Ground } \\
\text { (SRM 3233) }\end{array}$ & $\begin{array}{c}\text { Cereal B } \\
\text { Wheat } \\
\text { Ground }\end{array}$ & $\begin{array}{c}\text { Cereal C } \\
\text { Wheat } \\
\text { Flake }\end{array}$ & $\begin{array}{c}\text { Cereal D } \\
\text { Rice } \\
\text { Ground }\end{array}$ & $\begin{array}{c}\text { Cereal E } \\
\text { Rice } \\
\text { Flake }\end{array}$ & $\begin{array}{c}\text { Cereal F } \\
\text { Wheat/Rice } \\
\text { Crushed }\end{array}$ \\
\hline $\mathrm{Fe}(\mu \mathrm{g} / \mathrm{g})$ & $861 \pm 21$ & $912 \pm 7$ & $938 \pm 13$ & $572 \pm 12$ & $577 \pm 16$ & $703 \pm 9$ \\
$\mathrm{Ca}(\mu \mathrm{g} / \mathrm{g})$ & $41730 \pm 560$ & $38720 \pm 220$ & $38890 \pm 650$ & $171 \pm 4$ & $161 \pm 6$ & $16120 \pm 540$ \\
$\mathrm{Zn}(\mu \mathrm{g} / \mathrm{g})$ & $675 \pm 7$ & $730 \pm 5$ & $743 \pm 10$ & $8 \pm 1$ & $7 \pm 1$ & $317 \pm 13$ \\
\hline
\end{tabular}


Figure Z. A consensus plot summarizing your individual results (Lab Code: E520)

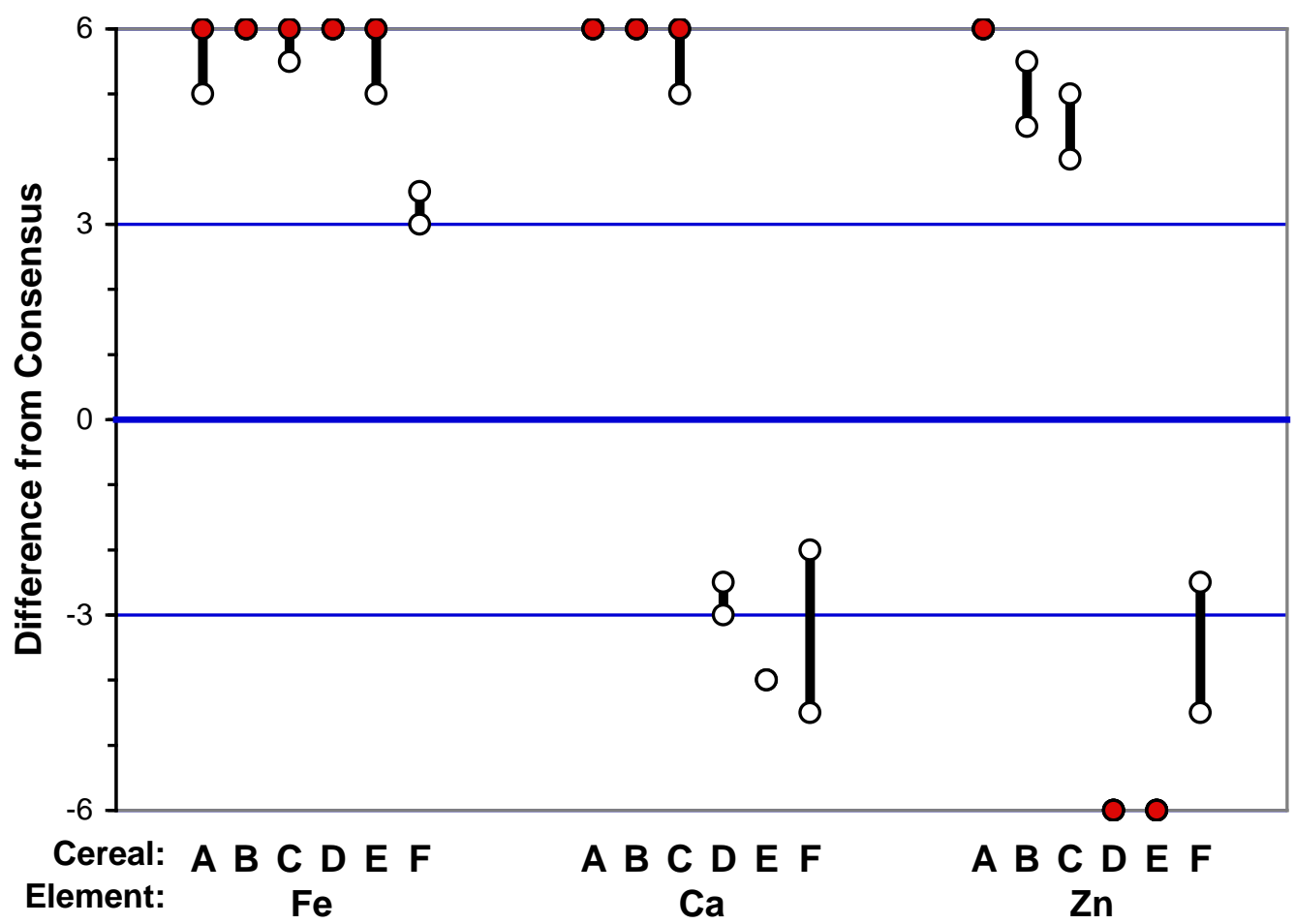

How to interpret your consensus summary plot:

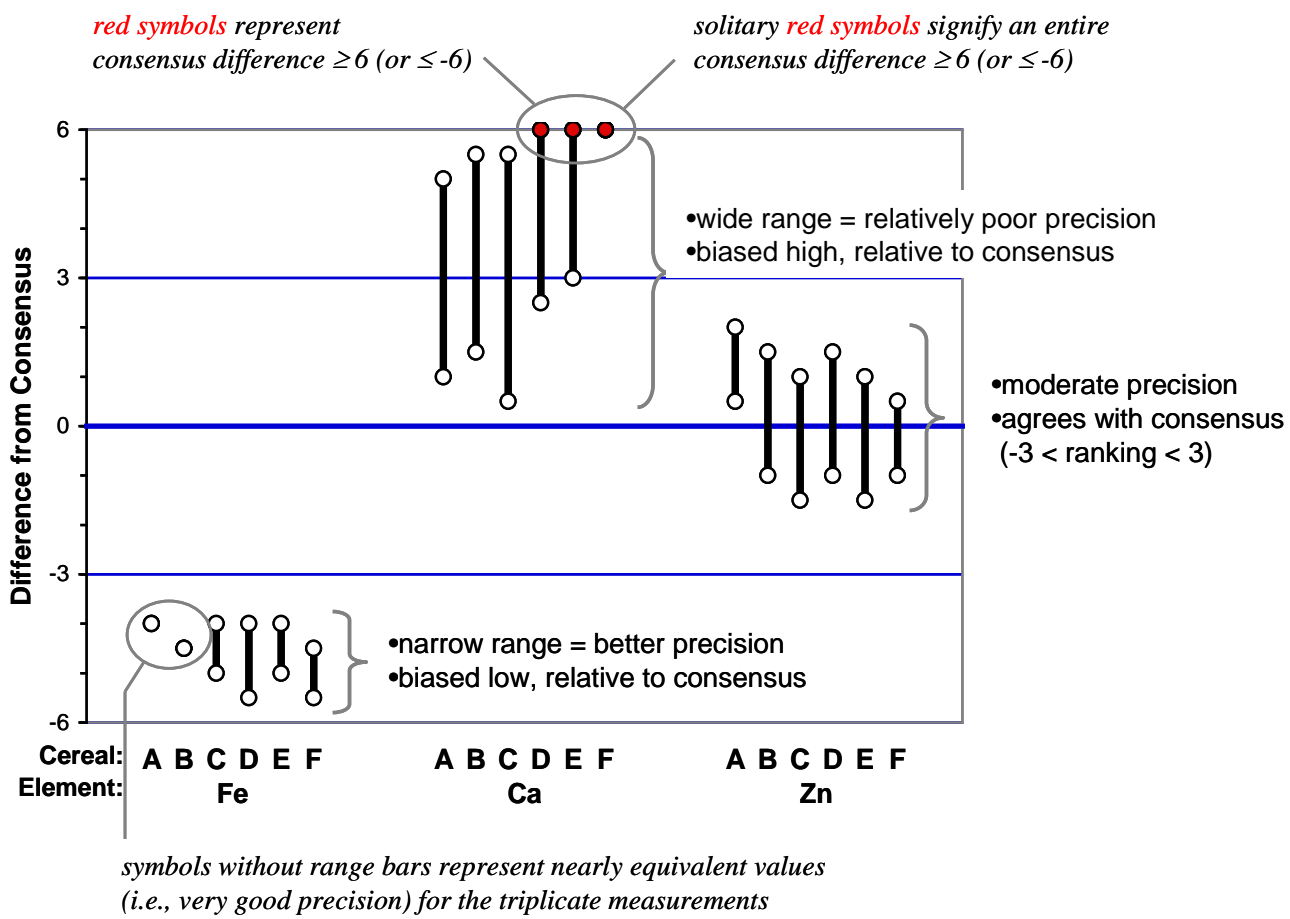




\section{Part II. Your Individual Laboratory Results (Lab Code: E522)}

Your laboratory's data is summarized in Table Z. The mean (average) \pm 1 standard deviation were determined from the reported triplicate $(n=3)$ values for each of the 18 measurements $(\mathrm{Fe}, \mathrm{Ca}$ and Zn within the six study materials, Cereals A-F) in Exercise E.

Your laboratory's summary consensus plot for $\mathrm{Fe}, \mathrm{Ca}$ and $\mathrm{Zn}$ in the six cereal study materials is provided in Figure Z. The data have been normalized relative to consensus means and Horn-HornDuncan (HHD) uncertainties so that the consensus value is equal to zero. The thick blue line in this plot represents the consensus values for each of the 18 measurements (Fe, $\mathrm{Ca}$ and $\mathrm{Zn}$ within the six study materials, Cereals A-F). The thin blue lines represent \pm 3 HHD standard uncertainty intervals about the consensus values. The figure is scaled so that the top and bottom of the Y-axis represent \pm 6 HHD intervals. Your laboratory's results for each of the 18 measurements are illustrated by the black bars. The ends of bars that are entirely within the \pm 6 HHD intervals are marked with an open circle (०); red circles $(\bullet)$ indicate that some or all of the bar is outside the \pm 6 HHD interval.

The length of each bar indicates how well your measurements agree with themselves. The distance from the center of the bars to the consensus line indicates how well your measurements agree with those of the other participants in this study. (An example consensus plot with explanations is also included to aid in the interpretation your results.) As a general rule, we consider results that fall within \pm 3 HHD standard uncertainty lines to be within consensus (approximate 99\% confidence limit) and results that have a black bar length $\approx 3$ HHD units are considered as acceptable precision. If values are consistently very high or low for a particular element, then a calibration problem may exist. In such cases, it is important to make sure your calibration standards are from a reliable source, the quality is confirmed and that your calibration solutions are correctly prepared and stored. In addition, calibration points must be linear in the expected measurement range and closely surround expected values.

Your laboratory's measurement results generally agree quite well with the consensus means, with the exception of the Ca values in the two rice-based cereals, D \& E. Given the relatively low amount of $\mathrm{Ca}$ in these rice materials, these low results suggest that the solutions analyzed may not have been in the linear region of your calibration curve. It is important to prepare samples to have appropriate mass fraction levels and to use enough calibration points to adequately cover the expected mass fraction range. Your results for all three elements in the wheat cereal (B \& C) and rice cereal (D \& E) pairs agree fairly well with each other, indicating a consistent sample preparation process. The precision of your measurement is quite good (bar lengths of 1 to 2 HHD units), with the exception of Fe in the ground wheat-based cereal B. This exception may possibly be due to a single contaminate sample or other unexplained outlier among two close values.

Table Z. Summary of Fe, Ca and Zn values within the six study materials, Cereals A-F

\begin{tabular}{|c|cccccc|}
\hline Lab Code: E522 & \multicolumn{5}{|c|}{ Mean Results $\pm \mathbf{1}$ SD (n= 3) } \\
\hline & $\begin{array}{c}\text { Cereal A } \\
\text { Wheat } \\
\text { Ground } \\
\text { (SRM 3233) }\end{array}$ & $\begin{array}{c}\text { Cereal B } \\
\text { Wheat } \\
\text { Ground }\end{array}$ & $\begin{array}{c}\text { Cereal C } \\
\text { Wheat } \\
\text { Flake }\end{array}$ & $\begin{array}{c}\text { Cereal D } \\
\text { Rice } \\
\text { Ground }\end{array}$ & $\begin{array}{c}\text { Cereal E } \\
\text { Rice } \\
\text { Flake }\end{array}$ & $\begin{array}{c}\text { Cereal F } \\
\text { Wheat/Rice } \\
\text { Crushed }\end{array}$ \\
\hline $\mathrm{Fe}(\mu \mathrm{g} / \mathrm{g})$ & $744 \pm 11$ & $787 \pm 43$ & $845 \pm 21$ & $492 \pm 8$ & $490 \pm 11$ & $653 \pm 6$ \\
$\mathrm{Ca}(\mu \mathrm{g} / \mathrm{g})$ & $37250 \pm 340$ & $35070 \pm 300$ & $34860 \pm 460$ & $145 \pm 4$ & $146 \pm 2$ & $17260 \pm 300$ \\
$\mathrm{Zn}(\mu \mathrm{g} / \mathrm{g})$ & $620 \pm 7$ & $688 \pm 6$ & $699 \pm 11$ & $19 \pm 0$ & $20 \pm 0.1$ & $358 \pm 4$ \\
\hline
\end{tabular}


Figure Z. A consensus plot summarizing your individual results (Lab Code: E522)

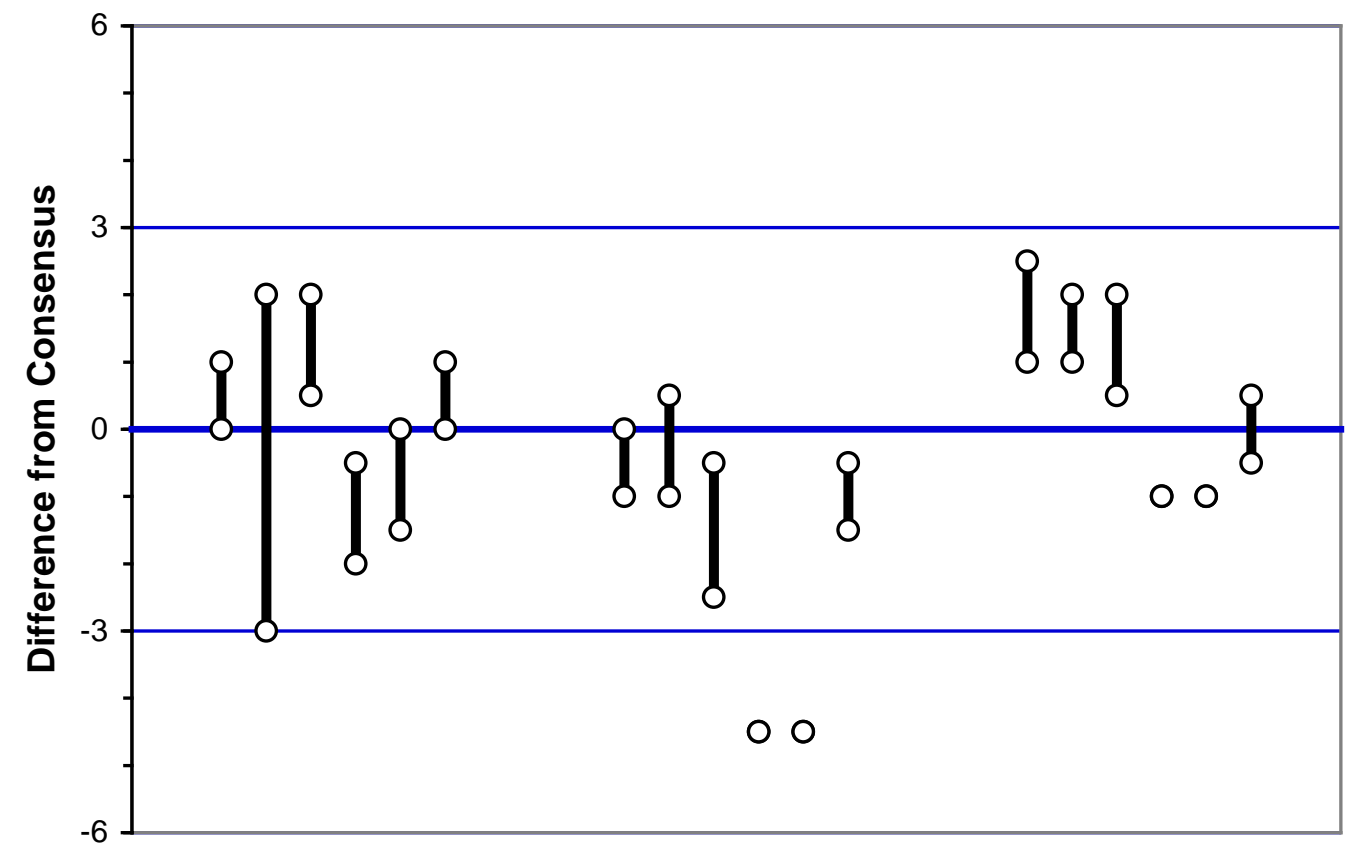
Cereal: A B C D E F
Element:
$\mathrm{Fe}$
$\underset{C a}{A}$ B $D E$
A B C D E F
$\mathrm{Zn}$

How to interpret your consensus summary plot:

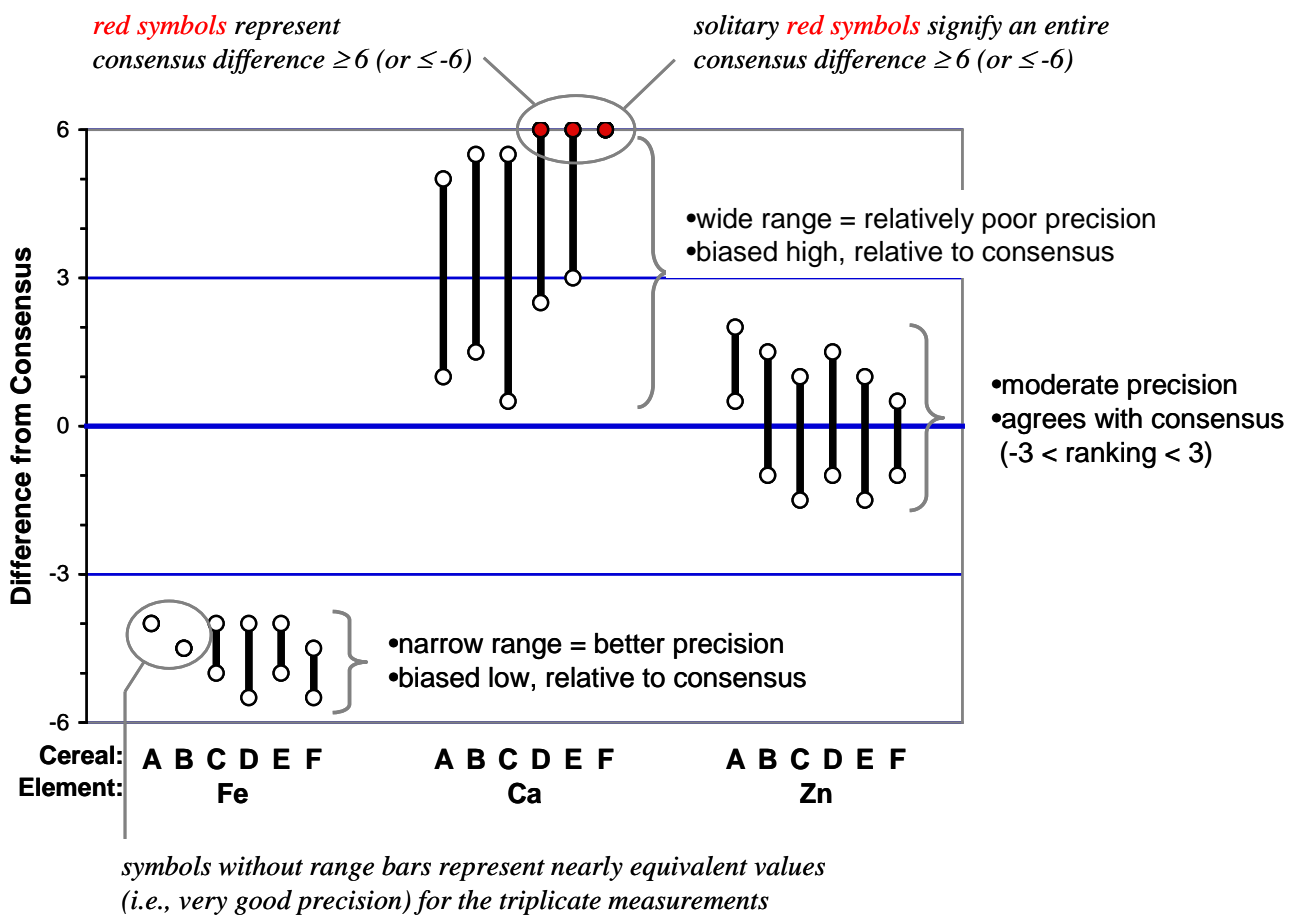




\section{Part II. Your Individual Laboratory Results (Lab Code: E526)}

Your laboratory's data is summarized in Table Z. The mean (average) \pm 1 standard deviation were determined from the reported triplicate $(\mathrm{n}=3$ ) values for each of the 18 measurements ( $\mathrm{Fe}, \mathrm{Ca}$ and $\mathrm{Zn}$ within the six study materials, Cereals A-F) in Exercise E.

Your laboratory's summary consensus plot for $\mathrm{Fe}, \mathrm{Ca}$ and $\mathrm{Zn}$ in the six cereal study materials is provided in Figure Z . The data have been normalized relative to consensus means and Horn-HornDuncan (HHD) uncertainties so that the consensus value is equal to zero. The thick blue line in this plot represents the consensus values for each of the 18 measurements ( $\mathrm{Fe}, \mathrm{Ca}$ and $\mathrm{Zn}$ within the six study materials, Cereals A-F). The thin blue lines represent \pm 3 HHD standard uncertainty intervals about the consensus values. The figure is scaled so that the top and bottom of the $Y$-axis represent \pm 6 HHD intervals. Your laboratory's results for each of the 18 measurements are illustrated by the black bars. The ends of bars that are entirely within the \pm 6 HHD intervals are marked with an open circle $(\circ)$; red circles $(\bullet)$ indicate that some or all of the bar is outside the \pm 6 HHD interval.

The length of each bar indicates how well your measurements agree with themselves. The distance from the center of the bars to the consensus line indicates how well your measurements agree with those of the other participants in this study. (An example consensus plot with explanations is also included to aid in the interpretation your results.) As a general rule, we consider results that fall within \pm 3 HHD standard uncertainty lines to be within consensus (approximate $99 \%$ confidence limit) and results that have a black bar length $\approx 3$ HHD units are considered as acceptable precision. If values are consistently very high or low for a particular element, then a calibration problem may exist. In such cases, it is important to make sure your calibration standards are from a reliable source, the quality is confirmed and that your calibration solutions are correctly prepared and stored. In addition, calibration points must be linear in the expected measurement range and closely surround expected values.

Your laboratory's measurement results for Fe and Zn in all six materials agree well with the consensus means. The Ca values are consistently slightly low, suggesting that you should check your $\mathrm{Ca}$ calibration standard. Your results for all three elements in the wheat cereal (B \& C) and rice cereal (D \& E) pairs agree fairly well with each other, indicating a consistent sample preparation process. The precision of your measurements is quite good (bar lengths mostly of 1 to 2 HHD units).

Table Z. Summary of Fe, Ca and Zn values within the six study materials, Cereals A-F

\begin{tabular}{|c|cccccc|}
\hline Lab Code: E526 & \multicolumn{5}{|c|}{ Mean Results $\pm \mathbf{1}$ SD (n= 3) } \\
\hline & $\begin{array}{c}\text { Cereal A } \\
\text { Wheat } \\
\text { Ground } \\
\text { SRM 3233) }\end{array}$ & $\begin{array}{c}\text { Cereal B } \\
\text { Wheat } \\
\text { Ground }\end{array}$ & $\begin{array}{c}\text { Cereal C } \\
\text { Wheat } \\
\text { Flake }\end{array}$ & $\begin{array}{c}\text { Cereal D } \\
\text { Rice } \\
\text { Ground }\end{array}$ & $\begin{array}{c}\text { Cereal E } \\
\text { Rice } \\
\text { Flake }\end{array}$ & $\begin{array}{c}\text { Cereal F } \\
\text { Wheat/Rice } \\
\text { Crushed }\end{array}$ \\
\hline $\mathrm{Fe}(\mu \mathrm{g} / \mathrm{g})$ & $747 \pm 6$ & $826 \pm 2$ & $848 \pm 43$ & $507 \pm 7$ & $501 \pm 5$ & $667 \pm 6$ \\
$\mathrm{Ca}(\mu \mathrm{g} / \mathrm{g})$ & $36180 \pm 60$ & $34420 \pm 130$ & $34780 \pm 80$ & $183 \pm 6$ & $190 \pm 0$ & $16430 \pm 180$ \\
$\mathrm{Zn}(\mu \mathrm{g} / \mathrm{g})$ & $608 \pm 11$ & $679 \pm 4$ & $709 \pm 7$ & $21 \pm 0.2$ & $20 \pm 0.3$ & $343 \pm 3$ \\
\hline
\end{tabular}


Figure Z. A consensus plot summarizing your individual results (Lab Code: E526)

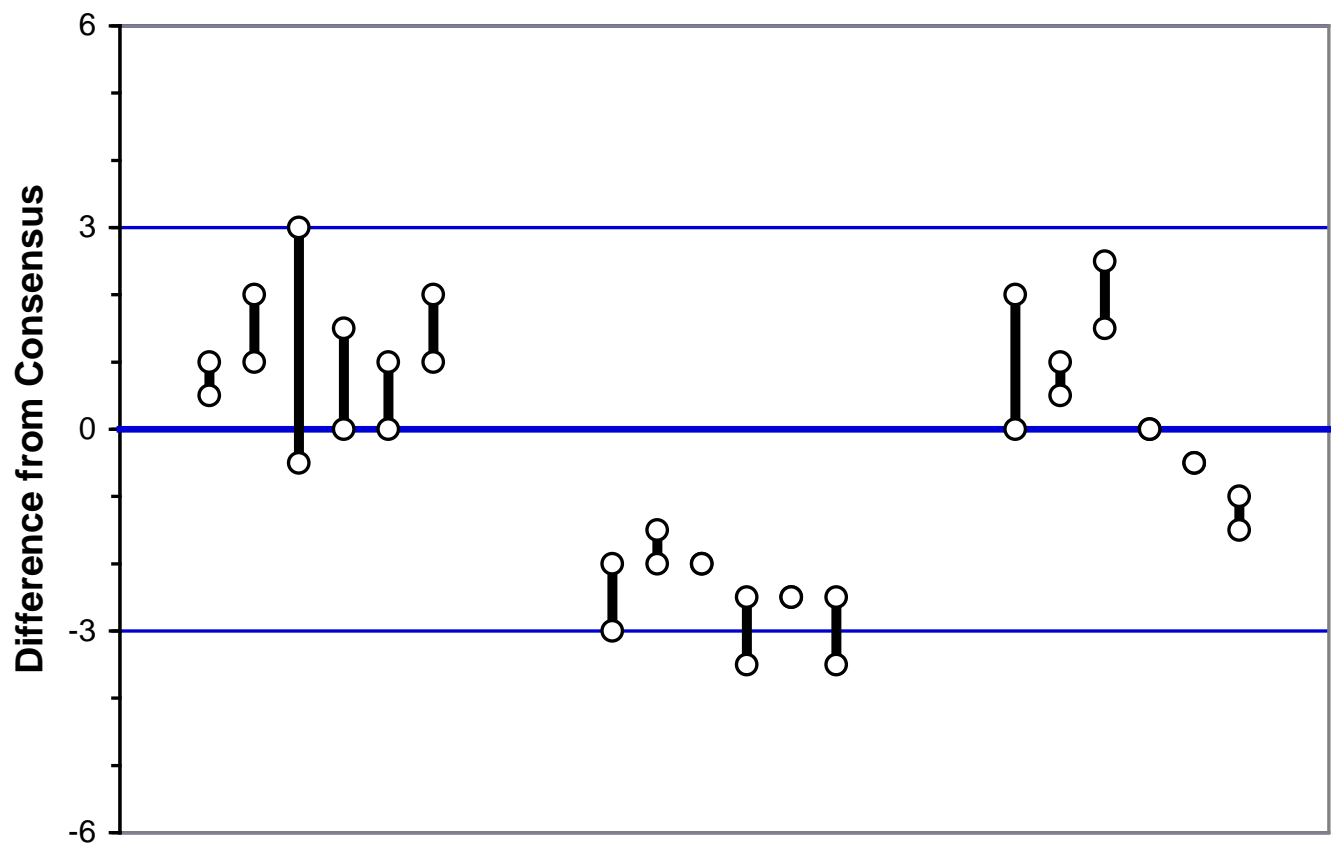
Cereal: A B C D E F
A B C D E F
A B C D E F
Element:
$\mathrm{Fe}$
$\mathrm{Ca}$
$\mathrm{Zn}$

How to interpret your consensus summary plot:

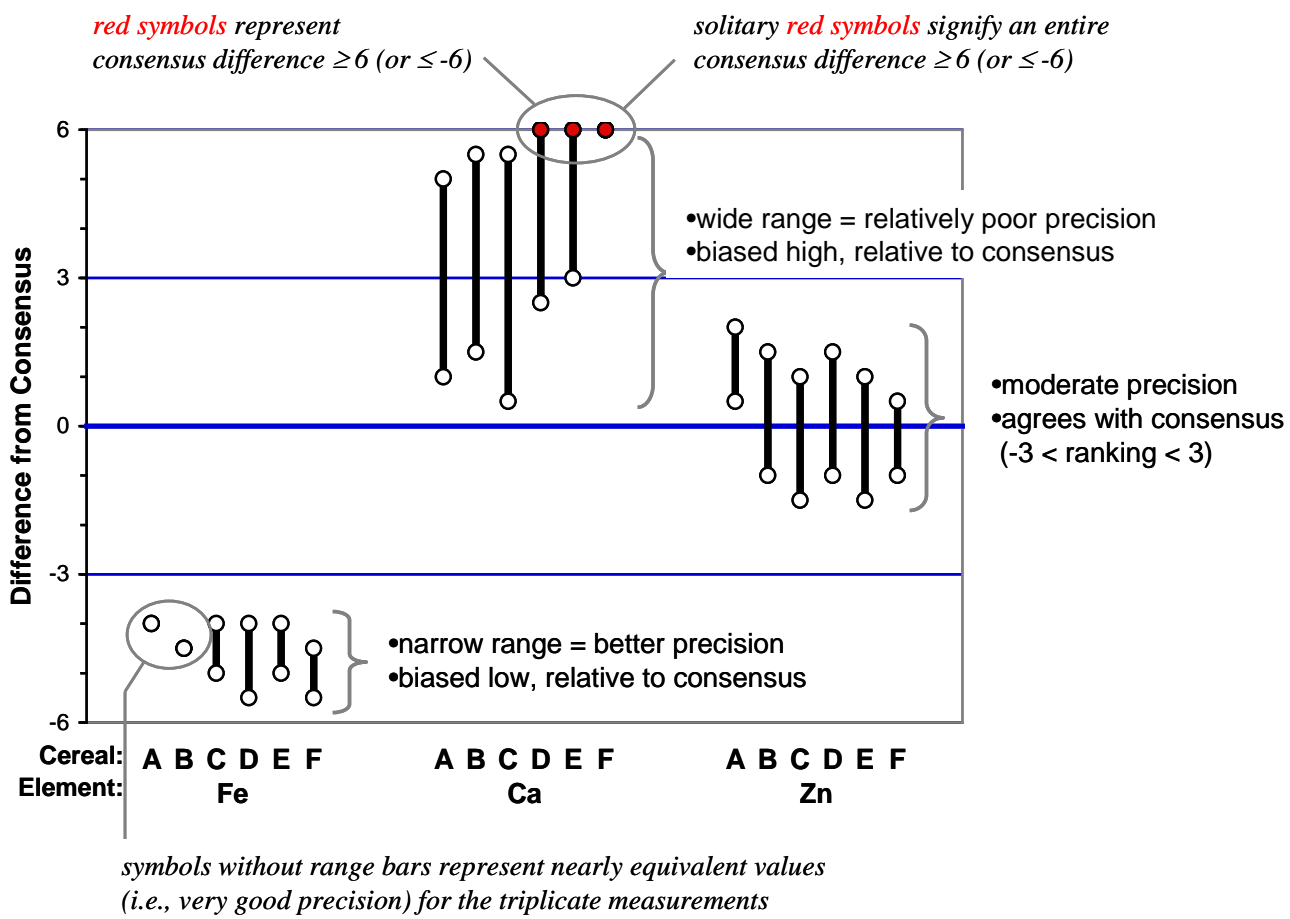




\section{Part II. Your Individual Laboratory Results (Lab Code: E529)}

Your laboratory's data is summarized in Table Z. The mean (average) \pm 1 standard deviation were determined from the reported triplicate $(n=3)$ values for each of the 18 measurements $(\mathrm{Fe}$, $\mathrm{Ca}$ and $\mathrm{Zn}$ within the six study materials, Cereals A-F) in Exercise E.

Your laboratory's summary consensus plot for $\mathrm{Fe}, \mathrm{Ca}$ and $\mathrm{Zn}$ in the six cereal study materials is provided in Figure Z. The data have been normalized relative to consensus means and Horn-HornDuncan (HHD) uncertainties so that the consensus value is equal to zero. The thick blue line in this plot represents the consensus values for each of the 18 measurements (Fe, $\mathrm{Ca}$ and $\mathrm{Zn}$ within the six study materials, Cereals A-F). The thin blue lines represent \pm 3 HHD standard uncertainty intervals about the consensus values. The figure is scaled so that the top and bottom of the $Y$-axis represent \pm 6 HHD intervals. Your laboratory's results for each of the 18 measurements are illustrated by the black bars. The ends of bars that are entirely within the \pm 6 HHD intervals are marked with an open circle $(\circ)$; red circles $(\bullet)$ indicate that some or all of the bar is outside the \pm 6 HHD interval.

The length of each bar indicates how well your measurements agree with themselves. The distance from the center of the bars to the consensus line indicates how well your measurements agree with those of the other participants in this study. (An example consensus plot with explanations is also included to aid in the interpretation your results.) As a general rule, we consider results that fall within \pm 3 HHD standard uncertainty lines to be within consensus (approximate 99\% confidence limit) and results that have a black bar length $\approx 3$ HHD units are considered as acceptable precision. If values are consistently very high or low for a particular element, then a calibration problem may exist. In such cases, it is important to make sure your calibration standards are from a reliable source, the quality is confirmed and that your calibration solutions are correctly prepared and stored. In addition, calibration points must be linear in the expected measurement range and closely surround expected values.

Your laboratory's measurement results for $\mathrm{Ca}$ and $\mathrm{Zn}$ (you did not report results for Fe) generally agree with the consensus means, indicating that your measurement processes are well calibrated. While your average results for $\mathrm{Zn}$ in the wheat-based cereal pair (B \& C) and for Ca in the rice-based pair ( $\mathrm{D} \& \mathrm{E}$ ) agree well, the results for $\mathrm{Zn}$ in $\mathrm{D} \& \mathrm{E}$ and Ca in B \& C do not agree well. The precision of your results is also somewhat variable, being rather good for the wheat-based materials (bar lengths of 1 to 2 HHD units for materials A, B \& C) but less good for the rice-based materials (some bars greater than 3 HDD units) and fairly poor for the mixed-source material F. These observations suggest that your sample preparation processes may not have sufficiently homogenized some of the sample materials or that sample digestions were incomplete.

Table Z. Summary of Ca and Zn values within the six study materials, Cereals A-F

\begin{tabular}{|c|cccccc|}
\hline Lab Code: E529 & \multicolumn{5}{|c|}{ Mean Results $\pm \mathbf{1}$ SD (n= 3) } \\
\hline & $\begin{array}{c}\text { Cereal A } \\
\text { Wheat } \\
\text { Ground } \\
(\text { SRM } 3233)\end{array}$ & $\begin{array}{c}\text { Cereal B } \\
\text { Wheat } \\
\text { Ground }\end{array}$ & $\begin{array}{c}\text { Cereal C } \\
\text { Wheat } \\
\text { Flake }\end{array}$ & $\begin{array}{c}\text { Cereal D } \\
\text { Rice } \\
\text { Ground }\end{array}$ & $\begin{array}{c}\text { Cereal E } \\
\text { Rice } \\
\text { Flake }\end{array}$ & $\begin{array}{c}\text { Cereal F } \\
\text { Wheat/Rice } \\
\text { Crushed }\end{array}$ \\
\hline $\mathrm{Ca}(\mu \mathrm{g} / \mathrm{g})$ & $38820 \pm 270$ & $34710 \pm 350$ & $37050 \pm 260$ & $201 \pm 45$ & $230 \pm 36$ & $18420 \pm 1500$ \\
$\mathrm{Zn}(\mu \mathrm{g} / \mathrm{g})$ & $588 \pm 6$ & $686 \pm 7$ & $682 \pm 7$ & $14 \pm 3$ & $19 \pm 0.1$ & $366 \pm 24$ \\
\hline
\end{tabular}


Figure Z. A consensus plot summarizing your individual results (Lab Code: E529)

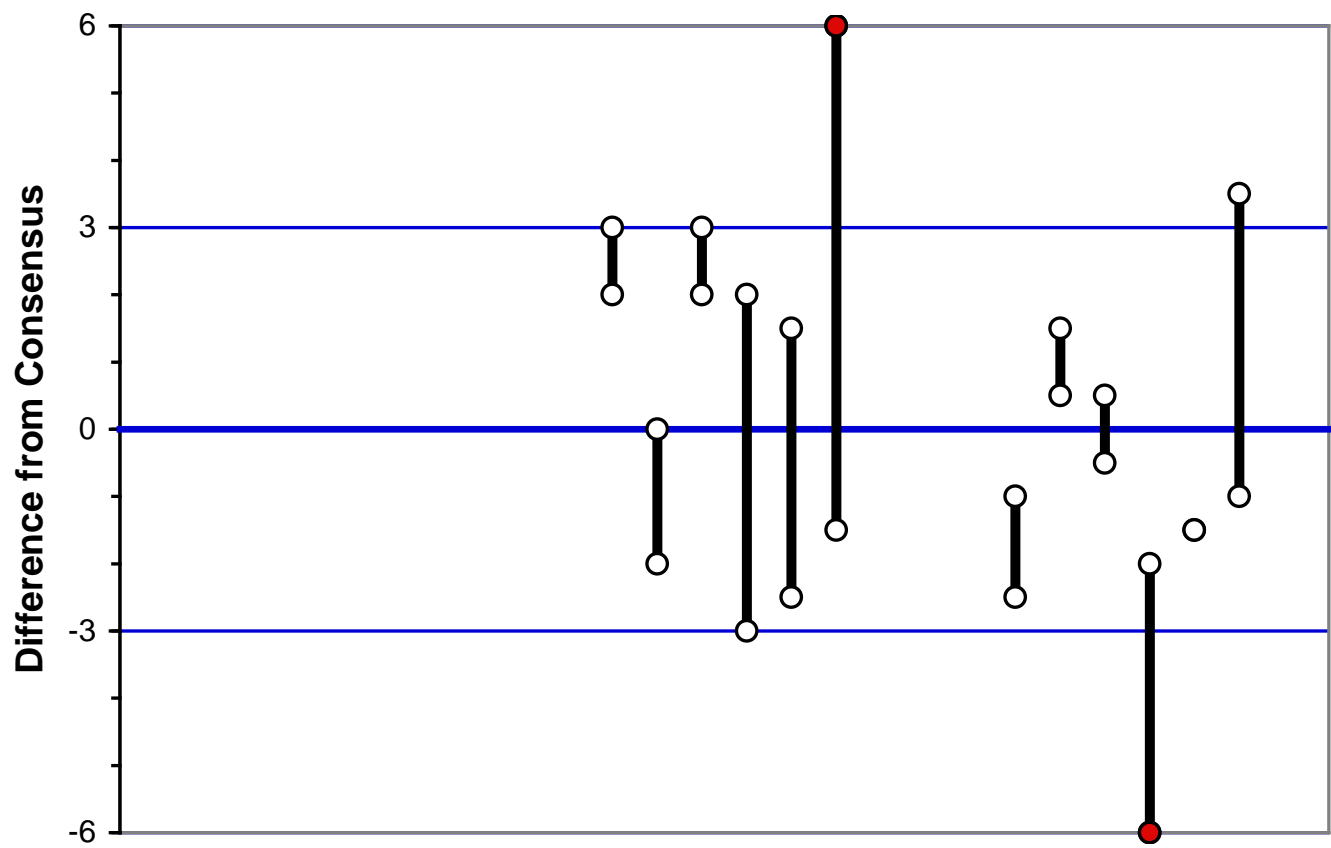
Cereal: A B C D E F
Element:
$\mathrm{Fe}$
A B C D E F
A B C D E F
$\mathrm{Zn}$

How to interpret your consensus summary plot:

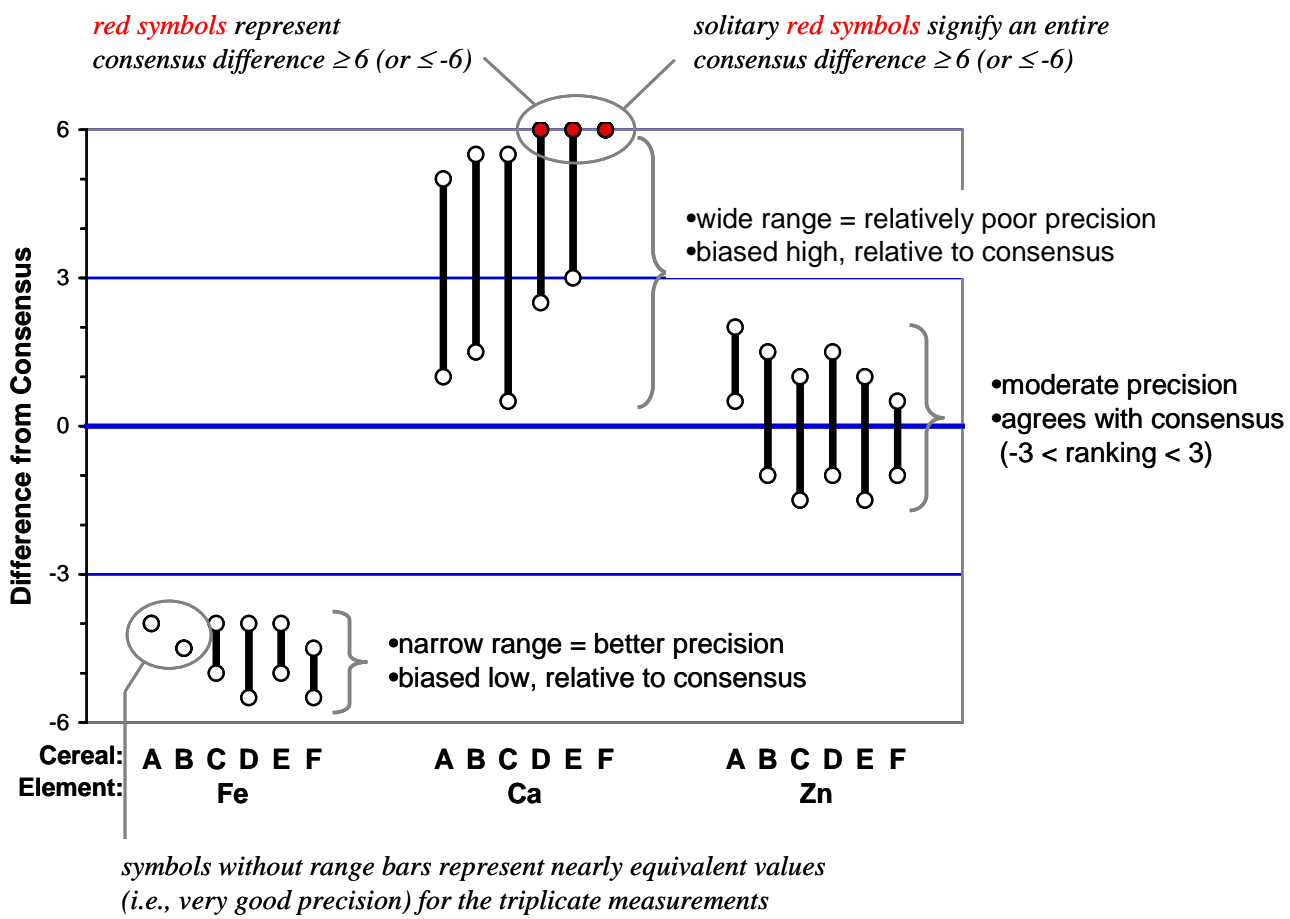




\section{Part II. Your Individual Laboratory Results (Lab Code: E530)}

Your laboratory's data is summarized in Table Z. The mean (average) \pm 1 standard deviation were determined from the reported triplicate $(\mathrm{n}=3$ ) values for each of the 18 measurements ( $\mathrm{Fe}, \mathrm{Ca}$ and $\mathrm{Zn}$ within the six study materials, Cereals A-F) in Exercise E.

Your laboratory's summary consensus plot for $\mathrm{Fe}, \mathrm{Ca}$ and $\mathrm{Zn}$ in the six cereal study materials is provided in Figure Z . The data have been normalized relative to consensus means and Horn-HornDuncan (HHD) uncertainties so that the consensus value is equal to zero. The thick blue line in this plot represents the consensus values for each of the 18 measurements ( $\mathrm{Fe}, \mathrm{Ca}$ and $\mathrm{Zn}$ within the six study materials, Cereals A-F). The thin blue lines represent \pm 3 HHD standard uncertainty intervals about the consensus values. The figure is scaled so that the top and bottom of the $Y$-axis represent \pm 6 HHD intervals. Your laboratory's results for each of the 18 measurements are illustrated by the black bars. The ends of bars that are entirely within the \pm 6 HHD intervals are marked with an open circle (०); red circles $(\bullet)$ indicate that some or all of the bar is outside the \pm 6 HHD interval.

The length of each bar indicates how well your measurements agree with themselves. The distance from the center of the bars to the consensus line indicates how well your measurements agree with those of the other participants in this study. (An example consensus plot with explanations is also included to aid in the interpretation your results.) As a general rule, we consider results that fall within \pm 3 HHD standard uncertainty lines to be within consensus (approximate $99 \%$ confidence limit) and results that have a black bar length $\approx 3$ HHD units are considered as acceptable precision. If values are consistently very high or low for a particular element, then a calibration problem may exist. In such cases, it is important to make sure your calibration standards are from a reliable source, the quality is confirmed and that your calibration solutions are correctly prepared and stored. In addition, calibration points must be linear in the expected measurement range and closely surround expected values.

Your laboratory's measurement results for Zn generally agree with the consensus means, indicating that your measurement process for this element is well calibrated. Your results for Fe fall consistently below the consensus values, with many less than -6 HHD. Iron is relatively easy to digest indicating a possible calibration problem and suggests that you should check your Fe calibration standards or a possible loss of Fe. You did not report results for $\mathrm{Ca}$. The results for Fe in the wheat-based cereal pair (B \& C) and the rice-based pair (D \& E) and Zn in the wheat-based cereal pair (B \& C) are not in good agreement, suggesting that your sample preparation process may not be consistently providing representative subsamples.

Table Z. Summary of Fe and Zn values within the six study materials, Cereals A-F

\begin{tabular}{|c|cccccc|}
\hline Lab Code: E530 & \multicolumn{7}{|c|}{ Mean Results $\pm \mathbf{1}$ SD $(\mathbf{n}=\mathbf{3})$} \\
\hline & $\begin{array}{c}\text { Cereal A } \\
\text { Wheat } \\
\text { Ground } \\
(\text { SRM 3233) }\end{array}$ & $\begin{array}{c}\text { Cereal B } \\
\text { Wheat } \\
\text { Ground }\end{array}$ & $\begin{array}{c}\text { Cereal C } \\
\text { Wheat } \\
\text { Flake }\end{array}$ & $\begin{array}{c}\text { Cereal D } \\
\text { Rice } \\
\text { Ground }\end{array}$ & $\begin{array}{c}\text { Cereal E } \\
\text { Rice } \\
\text { Flake }\end{array}$ & $\begin{array}{c}\text { Cereal F } \\
\text { Wheat/Rice } \\
\text { Crushed }\end{array}$ \\
\hline $\mathrm{Fe}(\mu \mathrm{g} / \mathrm{g})$ & $375 \pm 35$ & $697 \pm 65$ & $439 \pm 50$ & $461 \pm 42$ & $332 \pm 31$ & $347 \pm 69$ \\
$\mathrm{Zn}(\mu \mathrm{g} / \mathrm{g})$ & $590 \pm 5$ & $674 \pm 3$ & $647 \pm 25$ & $23 \pm 3$ & $21 \pm 1$ & $319 \pm 7$ \\
\hline
\end{tabular}


Figure Z. A consensus plot summarizing your individual results (Lab Code: E530)

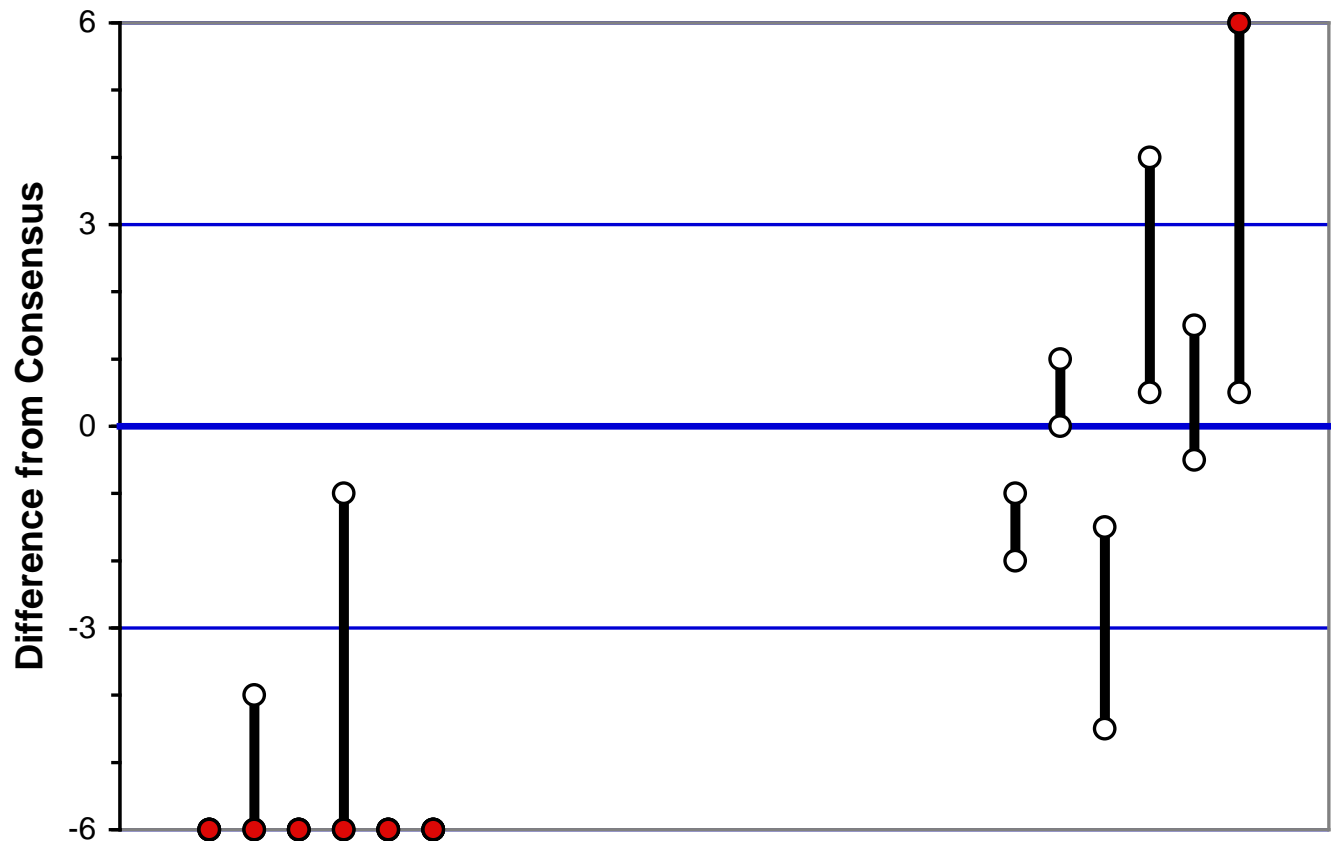
Cereal: A B C D E F
Element:
$\mathrm{Fe}$
$\underset{C a}{A}$ B $D E$
A B C D E F
$\mathrm{Zn}$

How to interpret your consensus summary plot:

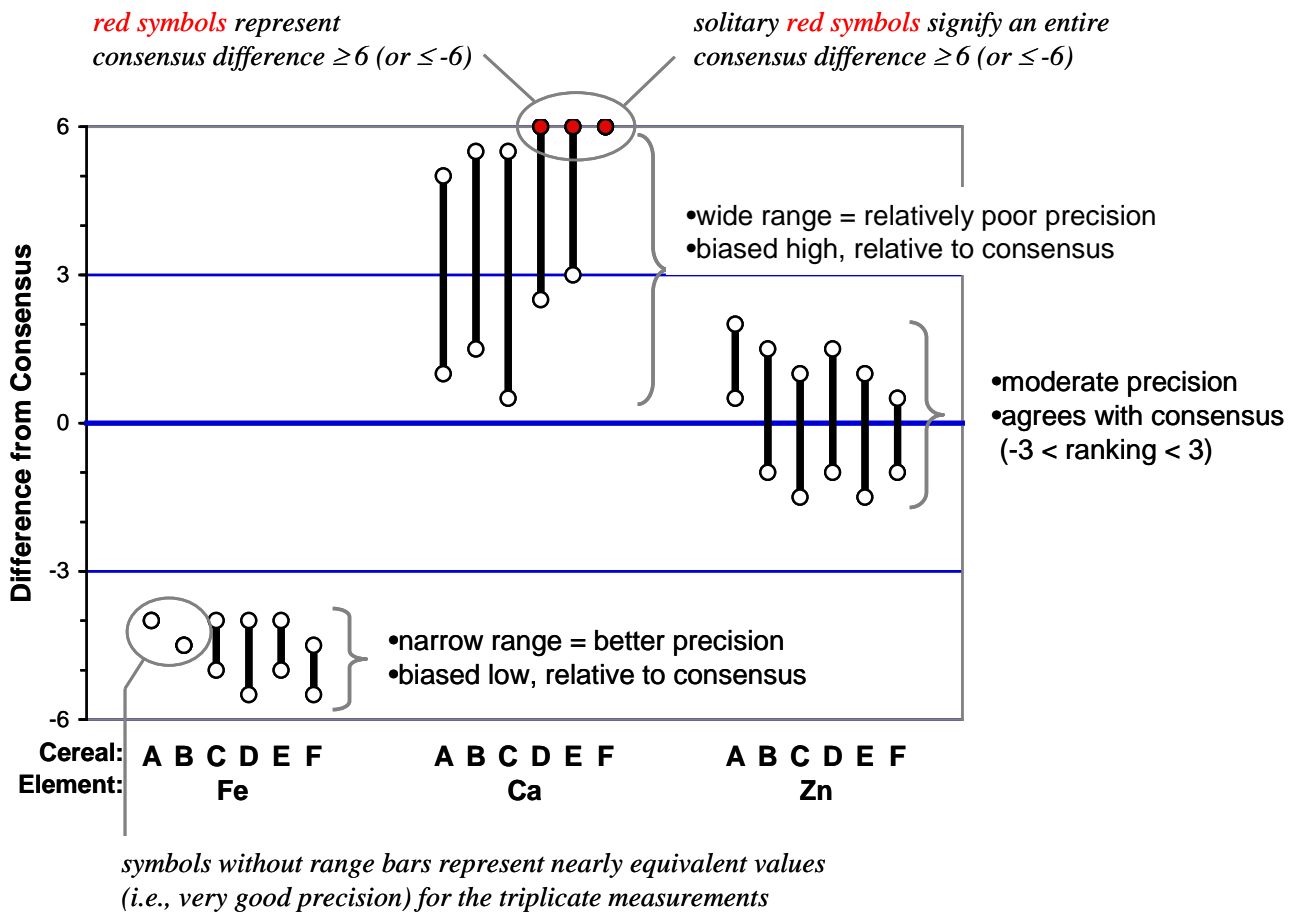




\section{Part II. Your Individual Laboratory Results (Lab Code: E531)}

Your laboratory's data is summarized in Table Z. The mean (average) \pm 1 standard deviation were determined from the reported triplicate $(\mathrm{n}=3$ ) values for each of the 18 measurements ( $\mathrm{Fe}, \mathrm{Ca}$ and $\mathrm{Zn}$ within the six study materials, Cereals A-F) in Exercise E.

Your laboratory's summary consensus plot for $\mathrm{Fe}, \mathrm{Ca}$ and $\mathrm{Zn}$ in the six cereal study materials is provided in Figure Z . The data have been normalized relative to consensus means and Horn-HornDuncan (HHD) uncertainties so that the consensus value is equal to zero. The thick blue line in this plot represents the consensus values for each of the 18 measurements ( $\mathrm{Fe}, \mathrm{Ca}$ and $\mathrm{Zn}$ within the six study materials, Cereals A-F). The thin blue lines represent \pm 3 HHD standard uncertainty intervals about the consensus values. The figure is scaled so that the top and bottom of the $Y$-axis represent \pm 6 HHD intervals. Your laboratory's results for each of the 18 measurements are illustrated by the black bars. The ends of bars that are entirely within the \pm 6 HHD intervals are marked with an open circle (०); red circles $(\bullet)$ indicate that some or all of the bar is outside the \pm 6 HHD interval.

The length of each bar indicates how well your measurements agree with themselves. The distance from the center of the bars to the consensus line indicates how well your measurements agree with those of the other participants in this study. (An example consensus plot with explanations is also included to aid in the interpretation your results.) As a general rule, we consider results that fall within \pm 3 HHD standard uncertainty lines to be within consensus (approximate $99 \%$ confidence limit) and results that have a black bar length $\approx 3$ HHD units are considered as acceptable precision. If values are consistently very high or low for a particular element, then a calibration problem may exist. In such cases, it is important to make sure your calibration standards are from a reliable source, the quality is confirmed and that your calibration solutions are correctly prepared and stored. In addition, calibration points must be linear in the expected measurement range and closely surround expected values.

Many of your laboratory's measurement results are rather imprecise (bar lengths of more than 3 HHD units). This imprecision may in part be related to your processing the materials after subsampling. You may want to investigate the use of subsampling and processing procedures that can more uniformly capture the bulk characteristics of the sampled materials (i.e. process entire sample before subsampling for analysis). Such imprecision for both ground and flake materials however may also suggest an incomplete digestion of samples.

Table Z. Summary of Fe, Ca and Zn values within the six study materials, Cereals A-F

\begin{tabular}{|c|cccccc|}
\hline Lab Code: E531 & \multicolumn{5}{|c|}{ Mean Results $\pm \mathbf{1}$ SD (n= 3) } \\
\hline & $\begin{array}{c}\text { Cereal A } \\
\text { Wheat } \\
\text { Ground } \\
\text { SRM 3233) }\end{array}$ & $\begin{array}{c}\text { Cereal B } \\
\text { Wheat } \\
\text { Ground }\end{array}$ & $\begin{array}{c}\text { Cereal C } \\
\text { Wheat } \\
\text { Flake }\end{array}$ & $\begin{array}{c}\text { Cereal D } \\
\text { Rice } \\
\text { Ground }\end{array}$ & $\begin{array}{c}\text { Cereal E } \\
\text { Rice } \\
\text { Flake }\end{array}$ & $\begin{array}{c}\text { Cereal F } \\
\text { Wheat/Rice } \\
\text { Crushed }\end{array}$ \\
\hline $\mathrm{Fe}(\mu \mathrm{g} / \mathrm{g})$ & $773 \pm 29$ & $823 \pm 87$ & $817 \pm 12$ & $513 \pm 21$ & $500 \pm 61$ & $707 \pm 35$ \\
$\mathrm{Ca}(\mu \mathrm{g} / \mathrm{g})$ & $38910 \pm 3290$ & $37240 \pm 2620$ & $37870 \pm 2140$ & $263 \pm 23$ & $213 \pm 23$ & $18010 \pm 1210$ \\
$\mathrm{Zn}(\mu \mathrm{g} / \mathrm{g})$ & $677 \pm 42$ & $793 \pm 40$ & $683 \pm 55$ & $20 \pm 0$ & $20 \pm 0$ & $427 \pm 21$ \\
\hline
\end{tabular}


Figure Z. A consensus plot summarizing your individual results (Lab Code: E531)

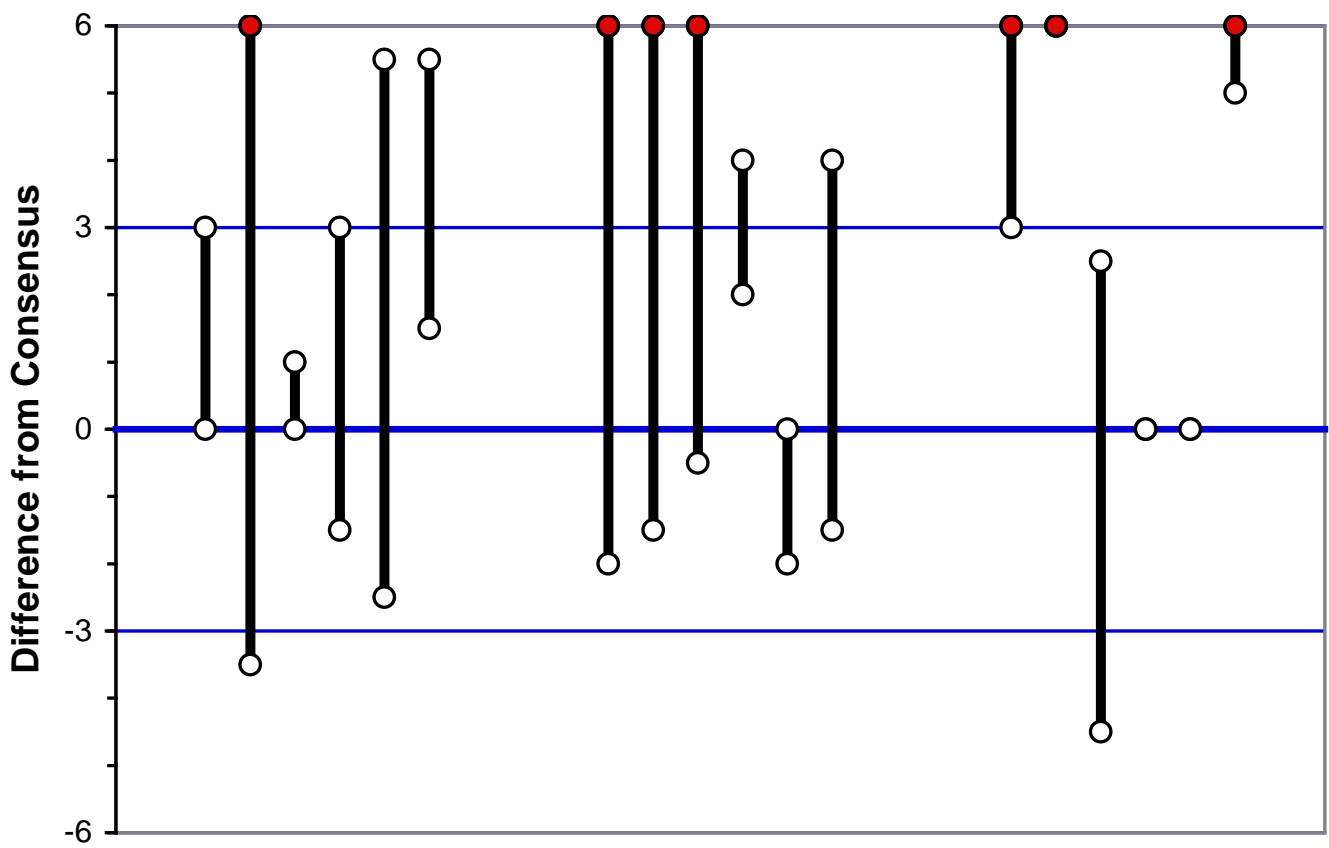
Cereal: A B C D E F
Element:
$\mathrm{Fe}$
A B C D E F
$\underset{Z n}{A}$ B E F

How to interpret your consensus summary plot:

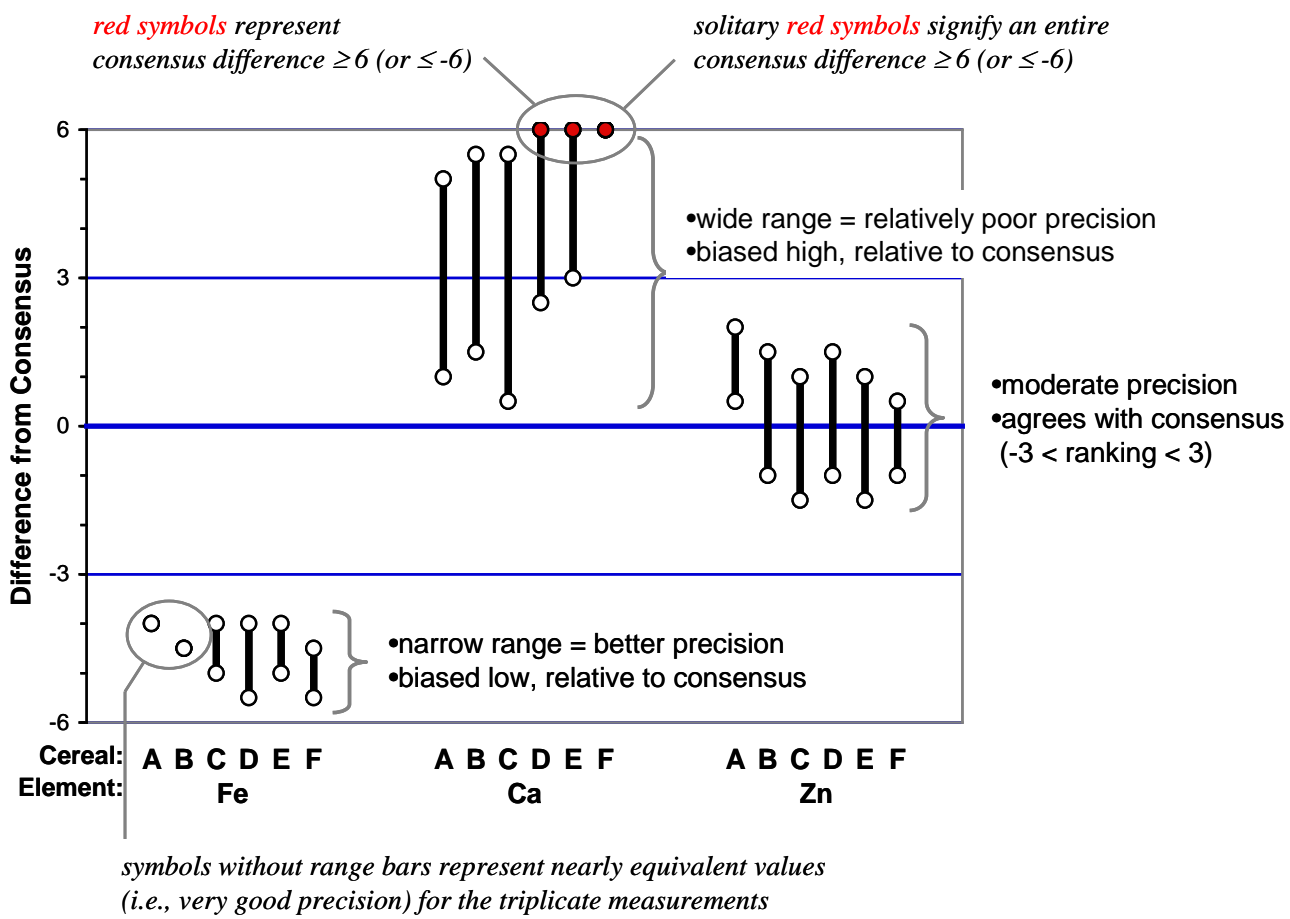




\section{Part II. Your Individual Laboratory Results (Lab Code: E533)}

Your laboratory's data is summarized in Table Z. The mean (average) \pm 1 standard deviation were determined from the reported triplicate $(n=3)$ values for each of the 18 measurements $(\mathrm{Fe}$, $\mathrm{Ca}$ and $\mathrm{Zn}$ within the six study materials, Cereals A-F) in Exercise E.

Your laboratory's summary consensus plot for $\mathrm{Fe}, \mathrm{Ca}$ and $\mathrm{Zn}$ in the six cereal study materials is provided in Figure Z. The data have been normalized relative to consensus means and Horn-HornDuncan (HHD) uncertainties so that the consensus value is equal to zero. The thick blue line in this plot represents the consensus values for each of the 18 measurements (Fe, $\mathrm{Ca}$ and $\mathrm{Zn}$ within the six study materials, Cereals A-F). The thin blue lines represent \pm 3 HHD standard uncertainty intervals about the consensus values. The figure is scaled so that the top and bottom of the $Y$-axis represent \pm 6 HHD intervals. Your laboratory's results for each of the 18 measurements are illustrated by the black bars. The ends of bars that are entirely within the \pm 6 HHD intervals are marked with an open circle $(\circ)$; red circles $(\bullet)$ indicate that some or all of the bar is outside the \pm 6 HHD interval.

The length of each bar indicates how well your measurements agree with themselves. The distance from the center of the bars to the consensus line indicates how well your measurements agree with those of the other participants in this study. (An example consensus plot with explanations is also included to aid in the interpretation your results.) As a general rule, we consider results that fall within \pm 3 HHD standard uncertainty lines to be within consensus (approximate $99 \%$ confidence limit) and results that have a black bar length $\approx 3$ HHD units are considered as acceptable precision. If values are consistently very high or low for a particular element, then a calibration problem may exist. In such cases, it is important to make sure your calibration standards are from a reliable source, the quality is confirmed and that your calibration solutions are correctly prepared and stored. In addition, calibration points must be linear in the expected measurement range and closely surround expected values.

Many of your laboratory's measurement results agree reasonably well with the consensus means, indicating that your measurement processes are adequately calibrated. However, the precision of your measurement processes is quite variable - ranging from quite good (bar lengths of $1 \mathrm{HHD}$ unit) to quite poor (bars longer than $6 \mathrm{HHD}$ units). In combination with somewhat inconsistent results for the flake/crush wheat (B \& C) and rice (D \& E) pairs, this suggests that your sample processing methods may not be consistently providing representative subsamples and/or that your sample digestion is not exhaustive.

Table Z. Summary of Fe, Ca and Zn values within the six study materials, Cereals A-F

\begin{tabular}{|c|cccccc|}
\hline Lab Code: E531 & \multicolumn{5}{|c|}{ Mean Results $\pm \mathbf{1}$ SD (n= 3) } \\
\hline & $\begin{array}{c}\text { Cereal A } \\
\text { Wheat } \\
\text { Ground } \\
\text { (SRM 3233) }\end{array}$ & $\begin{array}{c}\text { Cereal B } \\
\text { Wheat } \\
\text { Ground }\end{array}$ & $\begin{array}{c}\text { Cereal C } \\
\text { Wheat } \\
\text { Flake }\end{array}$ & $\begin{array}{c}\text { Cereal D } \\
\text { Rice } \\
\text { Ground }\end{array}$ & $\begin{array}{c}\text { Cereal E } \\
\text { Rice } \\
\text { Flake }\end{array}$ & $\begin{array}{c}\text { Cereal F } \\
\text { Wheat/Rice } \\
\text { Crushed }\end{array}$ \\
\hline $\mathrm{Fe}(\mu \mathrm{g} / \mathrm{g})$ & $690 \pm 20$ & $753 \pm 29$ & $793 \pm 6$ & $460 \pm 10$ & $467 \pm 12$ & $583 \pm 32$ \\
$\mathrm{Ca}(\mu \mathrm{g} / \mathrm{g})$ & $37530 \pm 250$ & $35100 \pm 170$ & $35430 \pm 720$ & $233 \pm 12$ & $217 \pm 6$ & $16400 \pm 2800$ \\
$\mathrm{Zn}(\mu \mathrm{g} / \mathrm{g})$ & $573 \pm 6$ & $650 \pm 36$ & $707 \pm 21$ & $30 \pm 0$ & $30 \pm 0$ & $327 \pm 50$ \\
\hline
\end{tabular}


Figure Z. A consensus plot summarizing your individual results (Lab Code: E533)

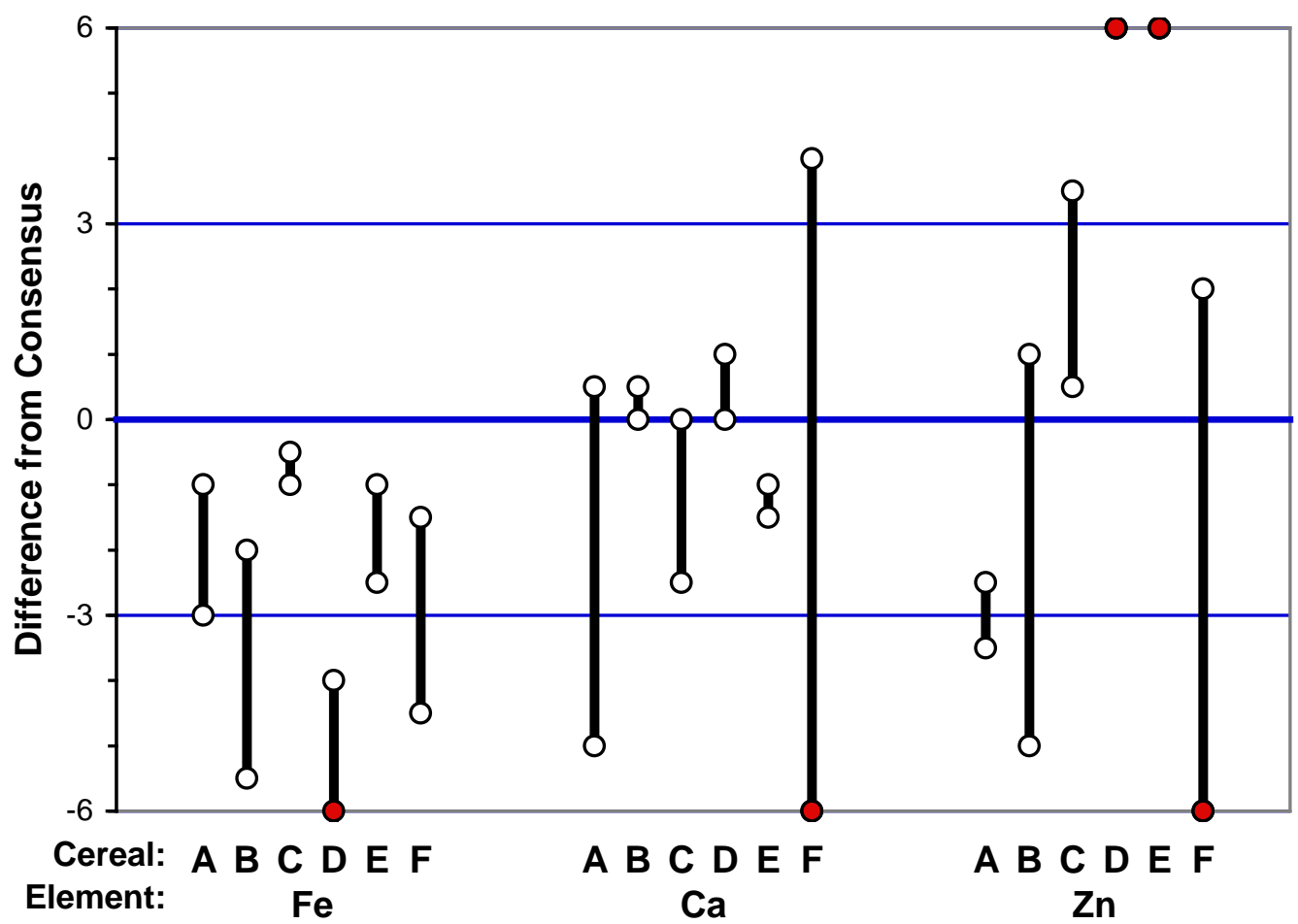

How to interpret your consensus summary plot:

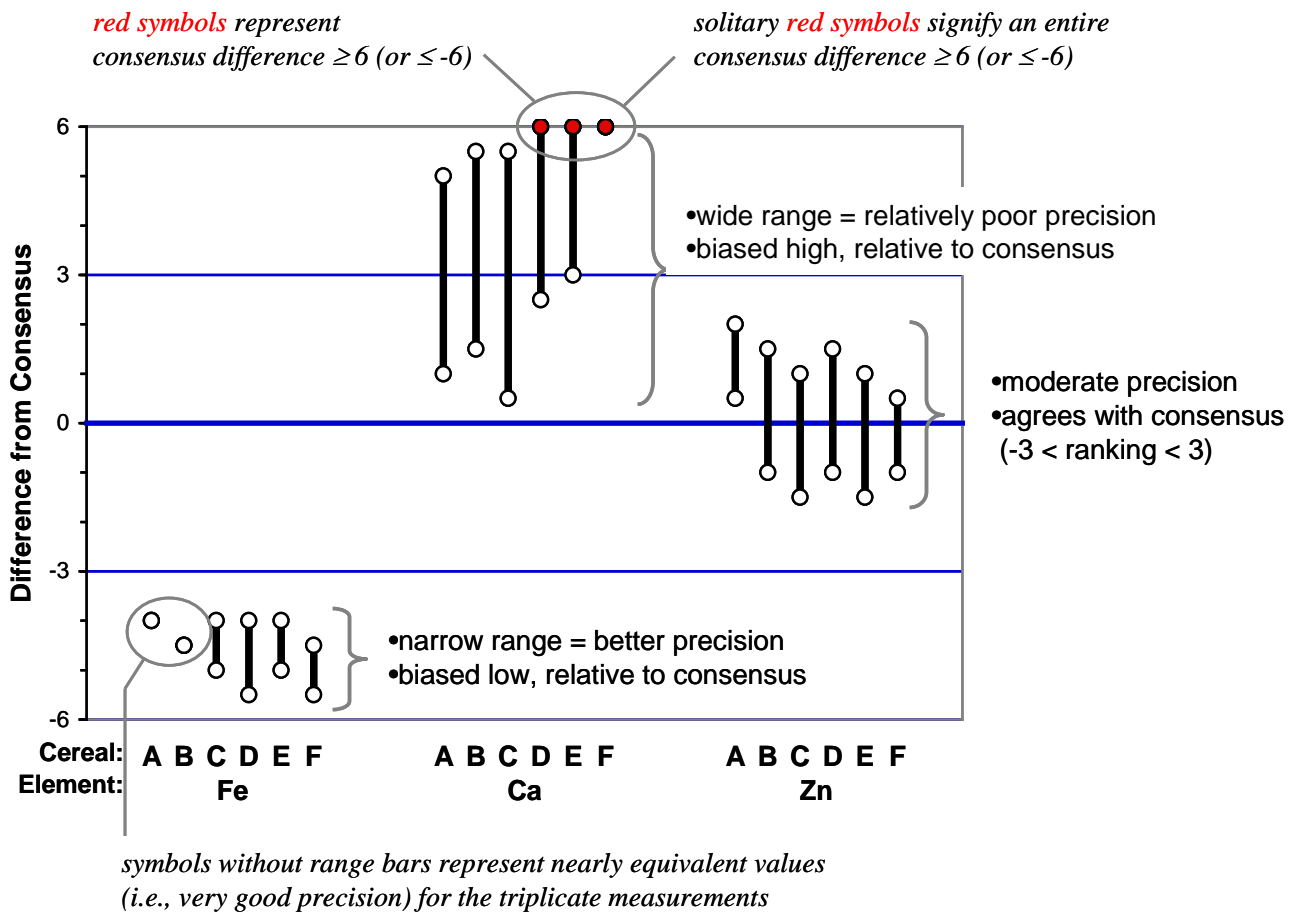




\section{Part II. Your Individual Laboratory Results (Lab Code: E534)}

Your laboratory's data is summarized in Table Z. The mean (average) \pm 1 standard deviation were determined from the reported triplicate $(n=3)$ values for each of the 18 measurements $(\mathrm{Fe}$, $\mathrm{Ca}$ and $\mathrm{Zn}$ within the six study materials, Cereals A-F) in Exercise E.

Your laboratory's summary consensus plot for $\mathrm{Fe}, \mathrm{Ca}$ and $\mathrm{Zn}$ in the six cereal study materials is provided in Figure Z. The data have been normalized relative to consensus means and Horn-HornDuncan (HHD) uncertainties so that the consensus value is equal to zero. The thick blue line in this plot represents the consensus values for each of the 18 measurements (Fe, $\mathrm{Ca}$ and $\mathrm{Zn}$ within the six study materials, Cereals A-F). The thin blue lines represent \pm 3 HHD standard uncertainty intervals about the consensus values. The figure is scaled so that the top and bottom of the $Y$-axis represent \pm 6 HHD intervals. Your laboratory's results for each of the 18 measurements are illustrated by the black bars. The ends of bars that are entirely within the \pm 6 HHD intervals are marked with an open circle $(\circ)$; red circles $(\bullet)$ indicate that some or all of the bar is outside the \pm 6 HHD interval.

The length of each bar indicates how well your measurements agree with themselves. The distance from the center of the bars to the consensus line indicates how well your measurements agree with those of the other participants in this study. (An example consensus plot with explanations is also included to aid in the interpretation your results.) As a general rule, we consider results that fall within \pm 3 HHD standard uncertainty lines to be within consensus (approximate $99 \%$ confidence limit) and results that have a black bar length $\approx 3$ HHD units are considered as acceptable precision. If values are consistently very high or low for a particular element, then a calibration problem may exist. In such cases, it is important to make sure your calibration standards are from a reliable source, the quality is confirmed and that your calibration solutions are correctly prepared and stored. In addition, calibration points must be linear in the expected measurement range and closely surround expected values.

Your laboratory's mean measurement values for Fe, Ca and $\mathrm{Zn}$ in the ground and flake wheat (B \& C) and rice (D \& E) cereal pairs agree fairly well and many of your measurement processes are adequately precise (bar lengths less than 3 HHD units), indicating a fairly consistent sample preparation process. Your results for Fe are consistently higher than the consensus means and the majority of Zn results are somewhat low; you may want to check your calibration standards for these elements. While your results for the relatively low-Ca content rice materials ( $\mathrm{D} \& \mathrm{E}$ ) agree well with the consensus values, your results for the relatively high-Ca wheat materials (A, B \& C) are a bit high. These results suggest that you may not be adequately matching sample mass fractions to the linear range of the calibration curve. It is important to prepare samples dilutions to have appropriate concentration levels and to use enough calibration points to adequately cover the expected concentration range.

Table Z. Summary of Fe, Ca and Zn values within the six study materials, Cereals A-F

\begin{tabular}{|c|cccccc|}
\hline Lab Code: E534 & \multicolumn{5}{|c|}{ Mean Results $\pm \mathbf{1}$ SD (n = 3) } \\
\hline & $\begin{array}{c}\text { Cereal A } \\
\text { Wheat } \\
\text { Ground } \\
\text { (SRM 3233) }\end{array}$ & $\begin{array}{c}\text { Cereal B } \\
\text { Wheat } \\
\text { Ground }\end{array}$ & $\begin{array}{c}\text { Cereal C } \\
\text { Wheat } \\
\text { Flake }\end{array}$ & $\begin{array}{c}\text { Cereal D } \\
\text { Rice } \\
\text { Ground }\end{array}$ & $\begin{array}{c}\text { Cereal E } \\
\text { Rice } \\
\text { Flake }\end{array}$ & $\begin{array}{c}\text { Cereal F } \\
\text { Wheat/Rice } \\
\text { Crushed }\end{array}$ \\
\hline $\mathrm{Fe}(\mu \mathrm{g} / \mathrm{g})$ & $849 \pm 32$ & $880 \pm 30$ & $900 \pm 11$ & $540 \pm 17$ & $537 \pm 19$ & $730 \pm 26$ \\
$\mathrm{Ca}(\mu \mathrm{g} / \mathrm{g})$ & $39700 \pm 530$ & $37000 \pm 1310$ & $37370 \pm 970$ & $224 \pm 19$ & $217 \pm 13$ & $20670 \pm 1300$ \\
$\mathrm{Zn}(\mu \mathrm{g} / \mathrm{g})$ & $582 \pm 4$ & $622 \pm 16$ & $601 \pm 16$ & $17 \pm 1$ & $17 \pm 1$ & $358 \pm 31$ \\
\hline
\end{tabular}


Figure Z. A consensus plot summarizing your individual results (Lab Code: E534)

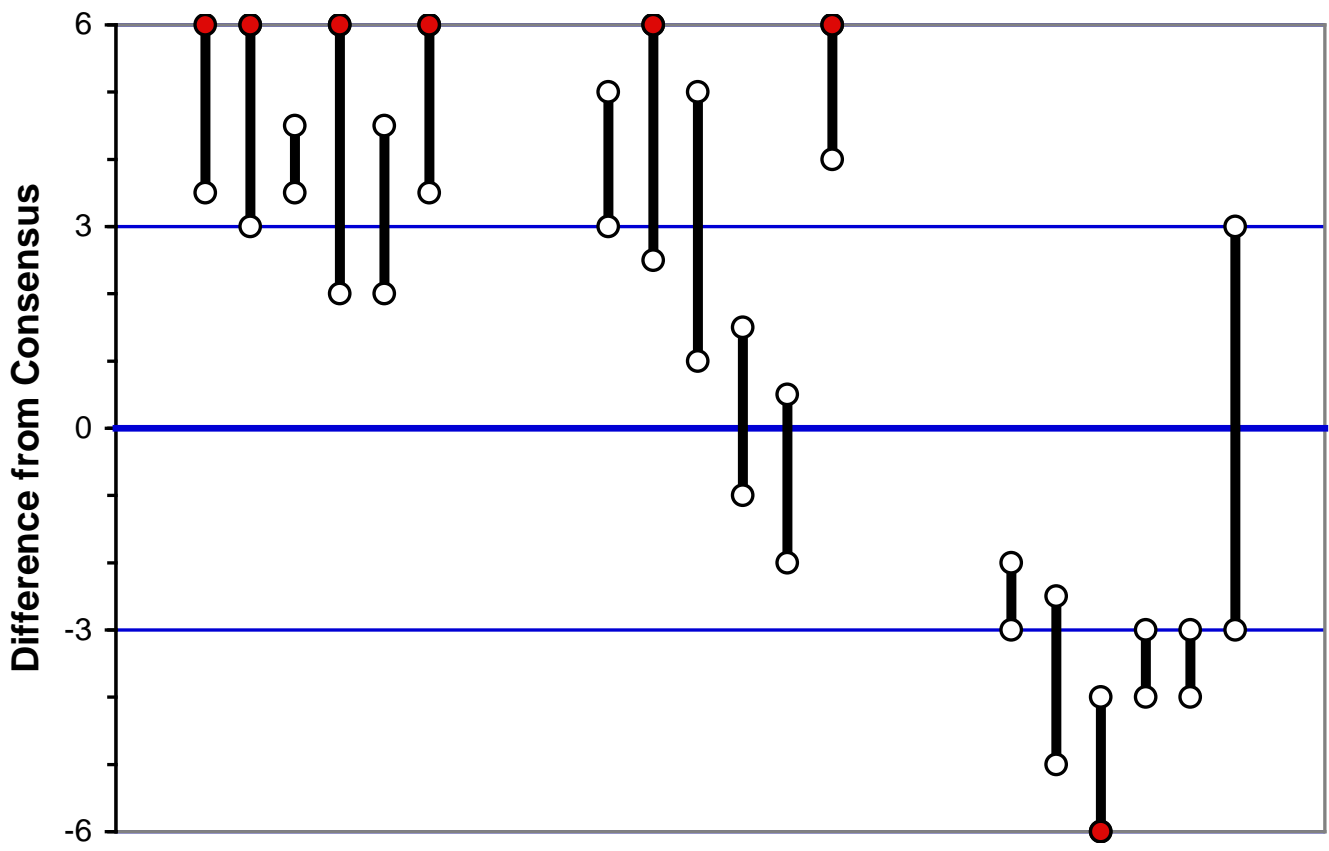
Cereal: A B C D E F
Element:
$\mathrm{Fe}$
$\underset{C a}{A}$ C D F
A B C D E F

How to interpret your consensus summary plot:

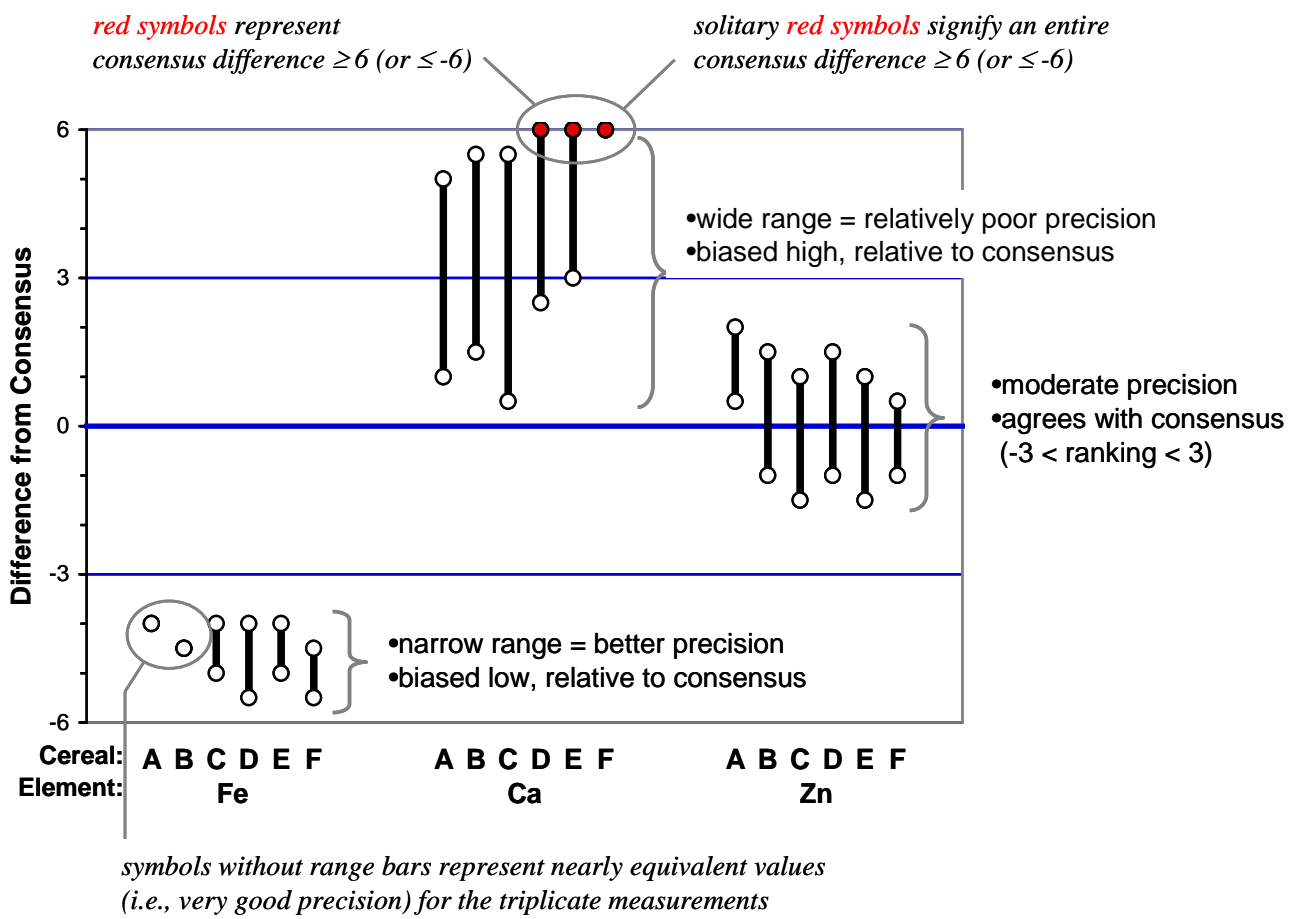




\section{Part II. Your Individual Laboratory Results (Lab Code: E537)}

Your laboratory's data is summarized in Table Z. The mean (average) \pm 1 standard deviation were determined from the reported triplicate $(n=3)$ values for each of the 18 measurements $(\mathrm{Fe}$, $\mathrm{Ca}$ and $\mathrm{Zn}$ within the six study materials, Cereals A-F) in Exercise E.

Your laboratory's summary consensus plot for $\mathrm{Fe}, \mathrm{Ca}$ and $\mathrm{Zn}$ in the six cereal study materials is provided in Figure Z. The data have been normalized relative to consensus means and Horn-HornDuncan (HHD) uncertainties so that the consensus value is equal to zero. The thick blue line in this plot represents the consensus values for each of the 18 measurements (Fe, $\mathrm{Ca}$ and $\mathrm{Zn}$ within the six study materials, Cereals A-F). The thin blue lines represent \pm 3 HHD standard uncertainty intervals about the consensus values. The figure is scaled so that the top and bottom of the $Y$-axis represent \pm 6 HHD intervals. Your laboratory's results for each of the 18 measurements are illustrated by the black bars. The ends of bars that are entirely within the \pm 6 HHD intervals are marked with an open circle $(\circ)$; red circles $(\bullet)$ indicate that some or all of the bar is outside the \pm 6 HHD interval.

The length of each bar indicates how well your measurements agree with themselves. The distance from the center of the bars to the consensus line indicates how well your measurements agree with those of the other participants in this study. (An example consensus plot with explanations is also included to aid in the interpretation your results.) As a general rule, we consider results that fall within \pm 3 HHD standard uncertainty lines to be within consensus (approximate $99 \%$ confidence limit) and results that have a black bar length $\approx 3$ HHD units are considered as acceptable precision. If values are consistently very high or low for a particular element, then a calibration problem may exist. In such cases, it is important to make sure your calibration standards are from a reliable source, the quality is confirmed and that your calibration solutions are correctly prepared and stored. In addition, calibration points must be linear in the expected measurement range and closely surround expected values.

Your laboratory's measurement results agree modestly well with the consensus values and are relatively precise (many of the bars are less than 2 HHD units long), indicating that your measurement processes are adequately calibrated and fairly consistent within each material. However, while your mean results for all three elements for the rice ground and flake cereal pair (D \& E) are quite similar, your results for $\mathrm{Ca}$ in the wheat ground and flake pair (B \& $\mathrm{C}$ ) are significantly different. Given the Ca measurement precision in the high-Ca wheat materials (A, B \& C) is much poorer than in the rice materials ( $\mathrm{D} \& \mathrm{E}$ ), this may indicate that you are not adequately matching sample mass fractions to the linear range of the calibration curve. It is important to prepare samples to have appropriate concentration levels and to use enough calibration points to adequately cover the expected concentration range.

Table Z. Summary of Fe, Ca and Zn values within the six study materials, Cereals A-F

\begin{tabular}{|c|cccccc|}
\hline Lab Code: E537 & \multicolumn{5}{|c|}{ Mean Results \pm 1 SD (n = 3) } \\
\hline & $\begin{array}{c}\text { Cereal A } \\
\text { Wheat } \\
\text { Ground } \\
\text { (SRM 3233) }\end{array}$ & $\begin{array}{c}\text { Cereal B } \\
\text { Wheat } \\
\text { Ground }\end{array}$ & $\begin{array}{c}\text { Cereal C } \\
\text { Wheat } \\
\text { Flake }\end{array}$ & $\begin{array}{c}\text { Cereal D } \\
\text { Rice } \\
\text { Ground }\end{array}$ & $\begin{array}{c}\text { Cereal E } \\
\text { Rice } \\
\text { Flake }\end{array}$ & $\begin{array}{c}\text { Cereal F } \\
\text { Wheat/Rice } \\
\text { Crushed }\end{array}$ \\
\hline $\mathrm{Fe}(\mu \mathrm{g} / \mathrm{g})$ & $716 \pm 8$ & $783 \pm 19$ & $746 \pm 14$ & $517 \pm 9$ & $513 \pm 5$ & $627 \pm 17$ \\
$\mathrm{Ca}(\mu \mathrm{g} / \mathrm{g})$ & $40620 \pm 970$ & $34170 \pm 780$ & $37850 \pm 760$ & $176 \pm 8$ & $171 \pm 3$ & $17360 \pm 230$ \\
$\mathrm{Zn}(\mu \mathrm{g} / \mathrm{g})$ & $619 \pm 3$ & $660 \pm 8$ & $653 \pm 11$ & $20 \pm 0.3$ & $20 \pm 0.3$ & $310 \pm 12$ \\
\hline
\end{tabular}


Figure Z. A consensus plot summarizing your individual results (Lab Code: E537)

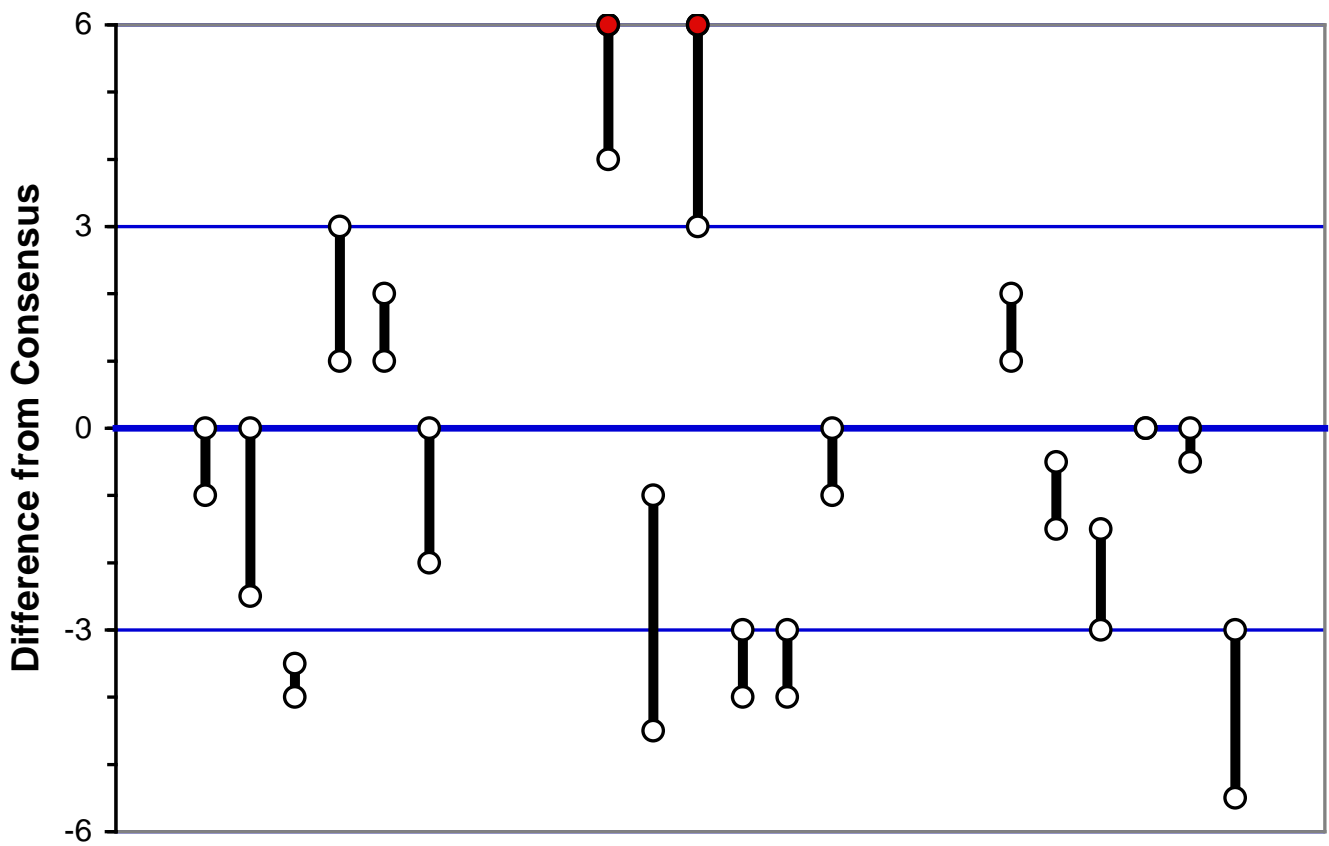
Cereal: A B C D E F
Element:
$\mathrm{Fe}$
$\underset{C a}{A}$ C D F
A B C DEF
$\mathrm{Zn}$

How to interpret your consensus summary plot:

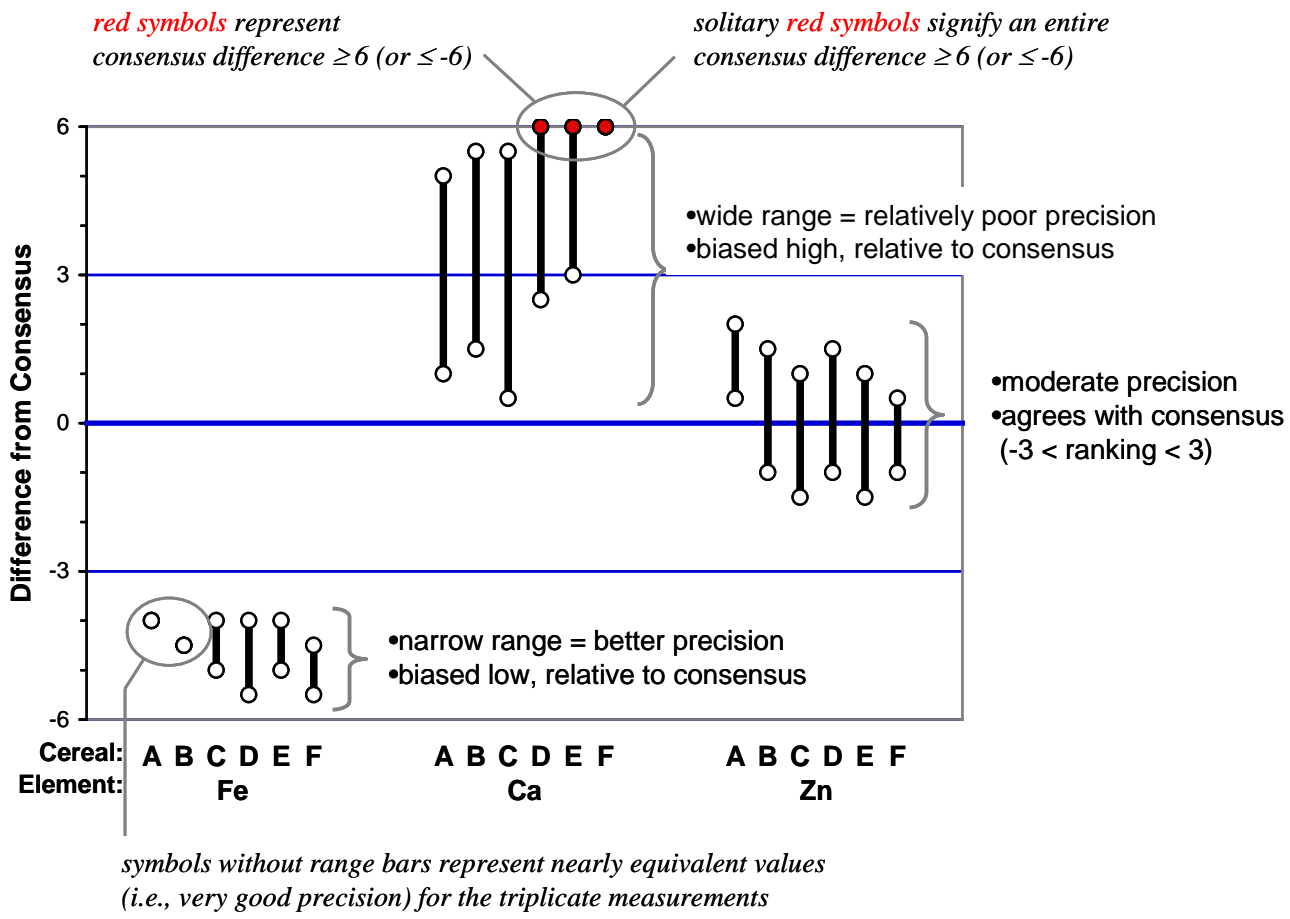




\section{Part II. Your Individual Laboratory Results (Lab Code: E538)}

Your laboratory's data is summarized in Table Z. The mean (average) \pm 1 standard deviation were determined from the reported triplicate $(n=3)$ values for each of the 18 measurements (Fe, $\mathrm{Ca}$ and $\mathrm{Zn}$ within the six study materials, Cereals A-F) in Exercise E.

Your laboratory's summary consensus plot for Fe, Ca and $\mathrm{Zn}$ in the six cereal study materials is provided in Figure Z $\mathbf{Z}$. The data have been normalized relative to consensus means and Horn-HornDuncan (HHD) uncertainties so that the consensus value is equal to zero. The thick blue line in this plot represents the consensus values for each of the 18 measurements (Fe, $\mathrm{Ca}$ and $\mathrm{Zn}$ within the six study materials, Cereals A-F). The thin blue lines represent \pm 3 HHD standard uncertainty intervals about the consensus values. The figure is scaled so that the top and bottom of the Y-axis represent \pm 6 HHD intervals. Your laboratory's results for each of the 18 measurements are illustrated by the black bars. The ends of bars that are entirely within the \pm 6 HHD intervals are marked with an open circle (०); red circles $(\bullet)$ indicate that some or all of the bar is outside the \pm 6 HHD interval.

The length of each bar indicates how well your measurements agree with themselves. The distance from the center of the bars to the consensus line indicates how well your measurements agree with those of the other participants in this study. (An example consensus plot with explanations is also included to aid in the interpretation your results.) As a general rule, we consider results that fall within \pm 3 HHD standard uncertainty lines to be within consensus (approximate 99\% confidence limit) and results that have a black bar length $\approx 3$ HHD units are considered as acceptable precision. If values are consistently very high or low for a particular element, then a calibration problem may exist. In such cases, it is important to make sure your calibration standards are from a reliable source, the quality is confirmed and that your calibration solutions are correctly prepared and stored. In addition, calibration points must be linear in the expected measurement range and closely surround expected values.

Your laboratory's measurement values for Zn are on average consistent with the consensus results; however your Fe results are consistently rather low. The precision of your measurement processes for all three elements is quite variable, ranging from good (bar lengths of 2 HHD units or less) to quite poor (bar lengths greater than 4 HHD units). This suggests that your sample preparation processes are inconsistent and your sample digestion process may not always be complete. You may want to investigate preparing samples from a larger subsample mass: the $1 \mathrm{~g}$ you reported using is small relative to that of other participants.

Table Z. Summary of Fe, Ca and Zn values within the six study materials, Cereals A-F

\begin{tabular}{|c|cccccc|}
\hline Lab Code: E538 & \multicolumn{5}{|c|}{ Mean Results $\pm \mathbf{1}$ SD (n= 3) } \\
\hline & $\begin{array}{c}\text { Cereal A } \\
\text { Wheat } \\
\text { Ground } \\
\text { (SRM 3233) }\end{array}$ & $\begin{array}{c}\text { Cereal B } \\
\text { Wheat } \\
\text { Ground }\end{array}$ & $\begin{array}{c}\text { Cereal C } \\
\text { Wheat } \\
\text { Flake }\end{array}$ & $\begin{array}{c}\text { Cereal D } \\
\text { Rice } \\
\text { Ground }\end{array}$ & $\begin{array}{c}\text { Cereal E } \\
\text { Rice } \\
\text { Flake }\end{array}$ & $\begin{array}{c}\text { Cereal F } \\
\text { Wheat/Rice } \\
\text { Crushed }\end{array}$ \\
\hline $\mathrm{Fe}(\mu \mathrm{g} / \mathrm{g})$ & $477 \pm 79$ & $556 \pm 107$ & $561 \pm 110$ & $440 \pm 54$ & $459 \pm 26$ & $524 \pm 49$ \\
$\mathrm{Ca}(\mu \mathrm{g} / \mathrm{g})$ & $36602 \pm 1530$ & $34320 \pm 1440$ & $34970 \pm 1480$ & $1248 \pm 314$ & $960 \pm 435$ & $19440 \pm 1760$ \\
$\mathrm{Zn}(\mu \mathrm{g} / \mathrm{g})$ & $577 \pm 23$ & $620 \pm 17$ & $627 \pm 28$ & $21 \pm 1$ & $20 \pm 1$ & $392 \pm 34$ \\
\hline
\end{tabular}


Figure Z. A consensus plot summarizing your individual results (Lab Code: E538)

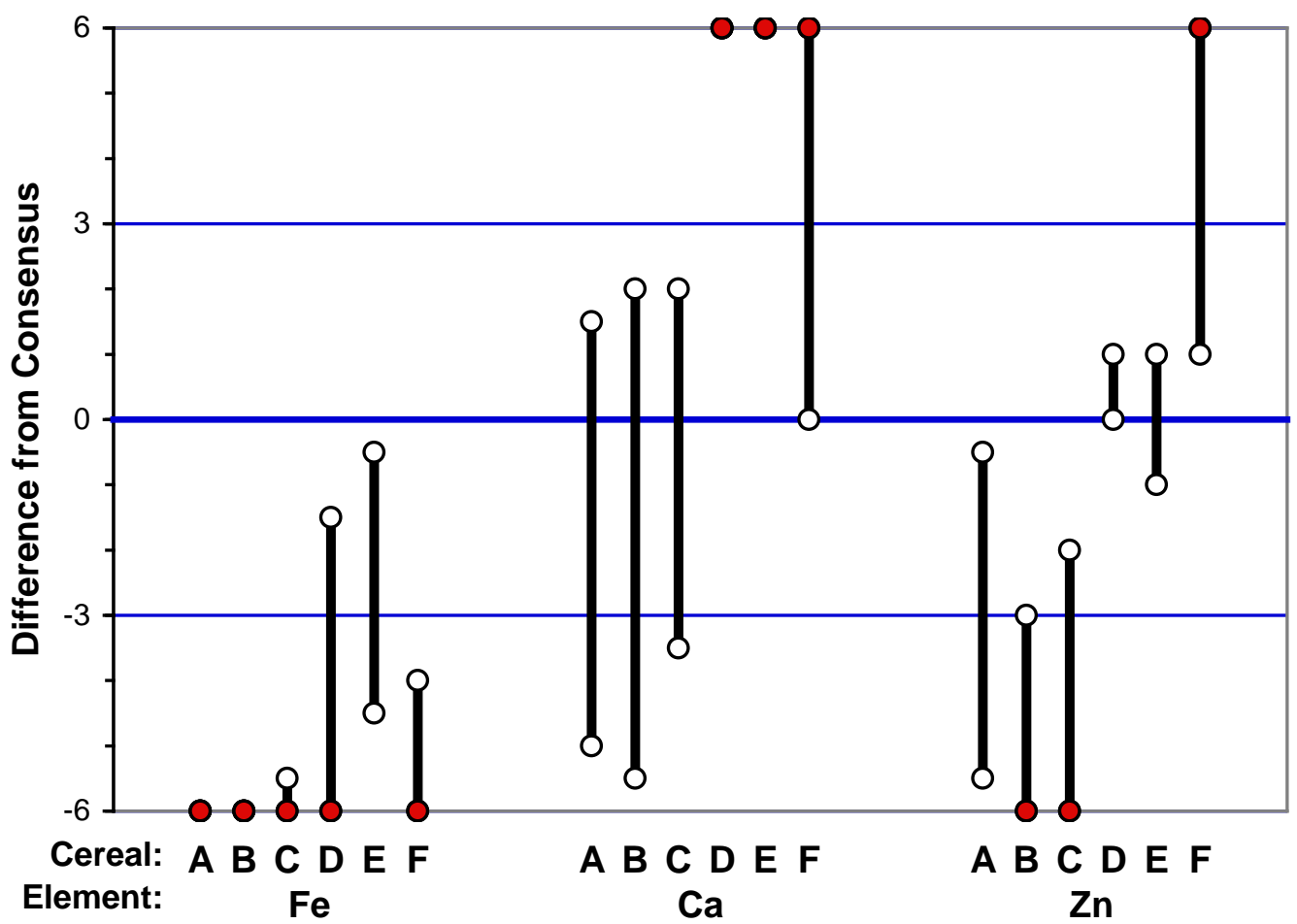

How to interpret your consensus summary plot:

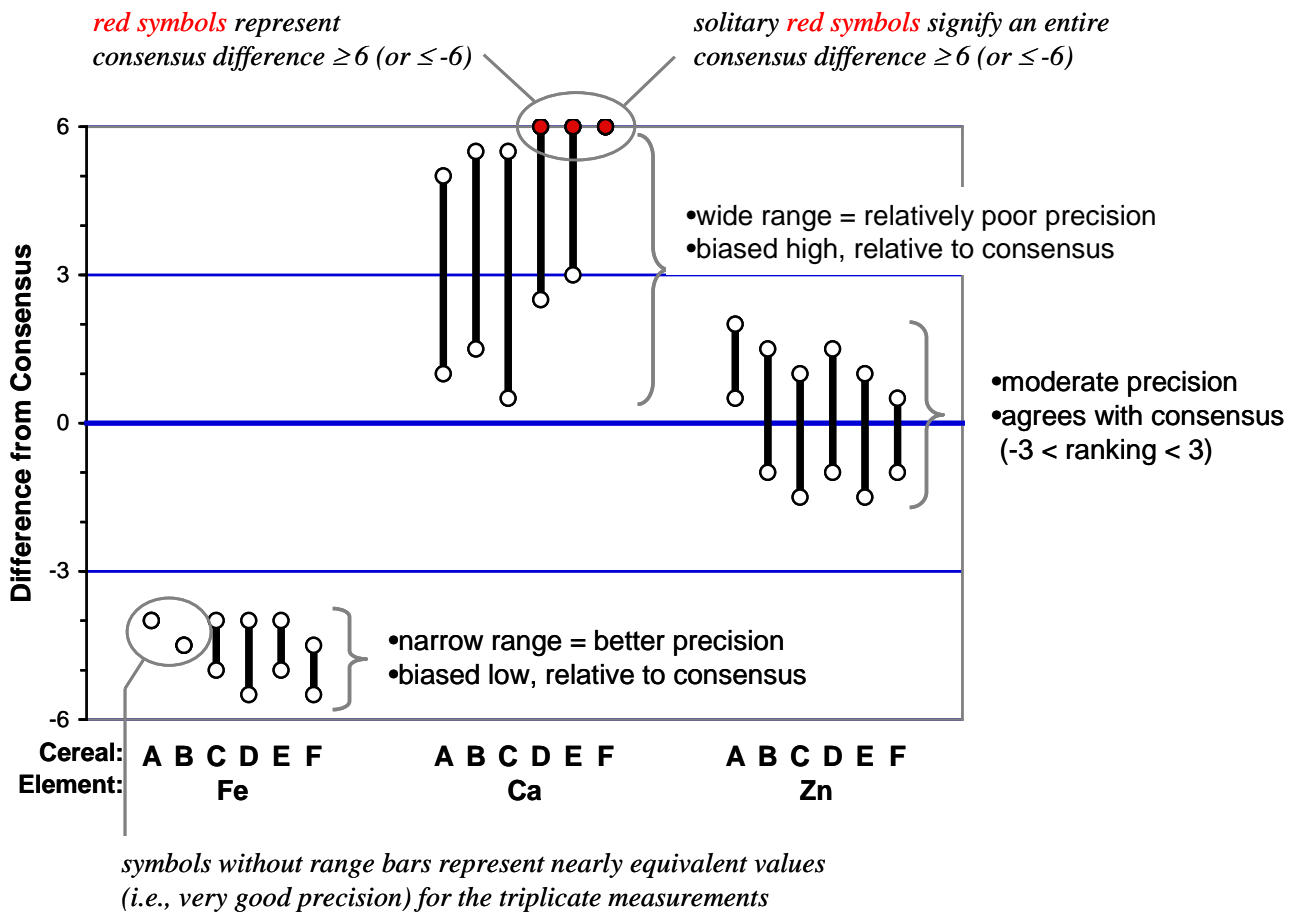




\section{Part II. Your Individual Laboratory Results (Lab Code: E539)}

Your laboratory's data is summarized in Table Z. The mean (average) \pm 1 standard deviation were determined from the reported triplicate $(n=3)$ values for each of the 18 measurements $(\mathrm{Fe}$, $\mathrm{Ca}$ and $\mathrm{Zn}$ within the six study materials, Cereals A-F) in Exercise E.

Your laboratory's summary consensus plot for $\mathrm{Fe}, \mathrm{Ca}$ and $\mathrm{Zn}$ in the six cereal study materials is provided in Figure Z. The data have been normalized relative to consensus means and Horn-HornDuncan (HHD) uncertainties so that the consensus value is equal to zero. The thick blue line in this plot represents the consensus values for each of the 18 measurements (Fe, $\mathrm{Ca}$ and $\mathrm{Zn}$ within the six study materials, Cereals A-F). The thin blue lines represent \pm 3 HHD standard uncertainty intervals about the consensus values. The figure is scaled so that the top and bottom of the Y-axis represent \pm 6 HHD intervals. Your laboratory's results for each of the 18 measurements are illustrated by the black bars. The ends of bars that are entirely within the \pm 6 HHD intervals are marked with an open circle (०); red circles $(\bullet)$ indicate that some or all of the bar is outside the \pm 6 HHD interval.

The length of each bar indicates how well your measurements agree with themselves. The distance from the center of the bars to the consensus line indicates how well your measurements agree with those of the other participants in this study. (An example consensus plot with explanations is also included to aid in the interpretation your results.) As a general rule, we consider results that fall within \pm 3 HHD standard uncertainty lines to be within consensus (approximate 99\% confidence limit) and results that have a black bar length $\approx 3$ HHD units are considered as acceptable precision. If values are consistently very high or low for a particular element, then a calibration problem may exist. In such cases, it is important to make sure your calibration standards are from a reliable source, the quality is confirmed and that your calibration solutions are correctly prepared and stored. In addition, calibration points must be linear in the expected measurement range and closely surround expected values.

Your laboratory's measurement results agree modestly well with the consensus values and are relatively precise (many of the bars are less than 2 HHD units long), indicating that your measurement processes are adequately calibrated and fairly consistently applied within each material. However, the extremely long bar for Fe in material D reflects an unusually large difference among the replicates and suggests that the consistency of your processes could be improved. Your results for the low-Ca rice ground and flake pair (D \& E) differ significantly for Ca and somewhat for Zn. This suggests that you may not be adequately matching the sample mass fractions to the linear range of the calibration curve. It is important to prepare samples to have appropriate concentration levels and to use calibration points that cover the expected concentration range.

Table Z. Summary of Fe, Ca and Zn values within the six study materials, Cereals A-F

\begin{tabular}{|c|cccccc|}
\hline Lab Code: E539 & \multicolumn{5}{|c|}{ Mean Results \pm 1 SD (n = 3) } \\
\hline & $\begin{array}{c}\text { Cereal A } \\
\text { Wheat } \\
\text { Ground } \\
\text { (SRM 3233) }\end{array}$ & $\begin{array}{c}\text { Cereal B } \\
\text { Wheat } \\
\text { Ground }\end{array}$ & $\begin{array}{c}\text { Cereal C } \\
\text { Wheat } \\
\text { Flake }\end{array}$ & $\begin{array}{c}\text { Cereal D } \\
\text { Rice } \\
\text { Ground }\end{array}$ & $\begin{array}{c}\text { Cereal E } \\
\text { Rice } \\
\text { Flake }\end{array}$ & $\begin{array}{c}\text { Cereal F } \\
\text { Wheat/Rice } \\
\text { Crushed }\end{array}$ \\
\hline $\mathrm{Fe}(\mu \mathrm{g} / \mathrm{g})$ & $782 \pm 11$ & $855 \pm 15$ & $850 \pm 40$ & $513 \pm 62$ & $571 \pm 35$ & $734 \pm 14$ \\
$\mathrm{Ca}(\mu \mathrm{g} / \mathrm{g})$ & $36700 \pm 460$ & $34930 \pm 990$ & $35500 \pm 610$ & $154 \pm 17$ & $390 \pm 91$ & $19730 \pm 1440$ \\
$\mathrm{Zn}(\mu \mathrm{g} / \mathrm{g})$ & $613 \pm 1$ & $684 \pm 7$ & $702 \pm 27$ & $19 \pm 1$ & $25 \pm 1$ & $416 \pm 34$ \\
\hline
\end{tabular}


Figure Z. A consensus plot summarizing your individual results (Lab Code: E539)

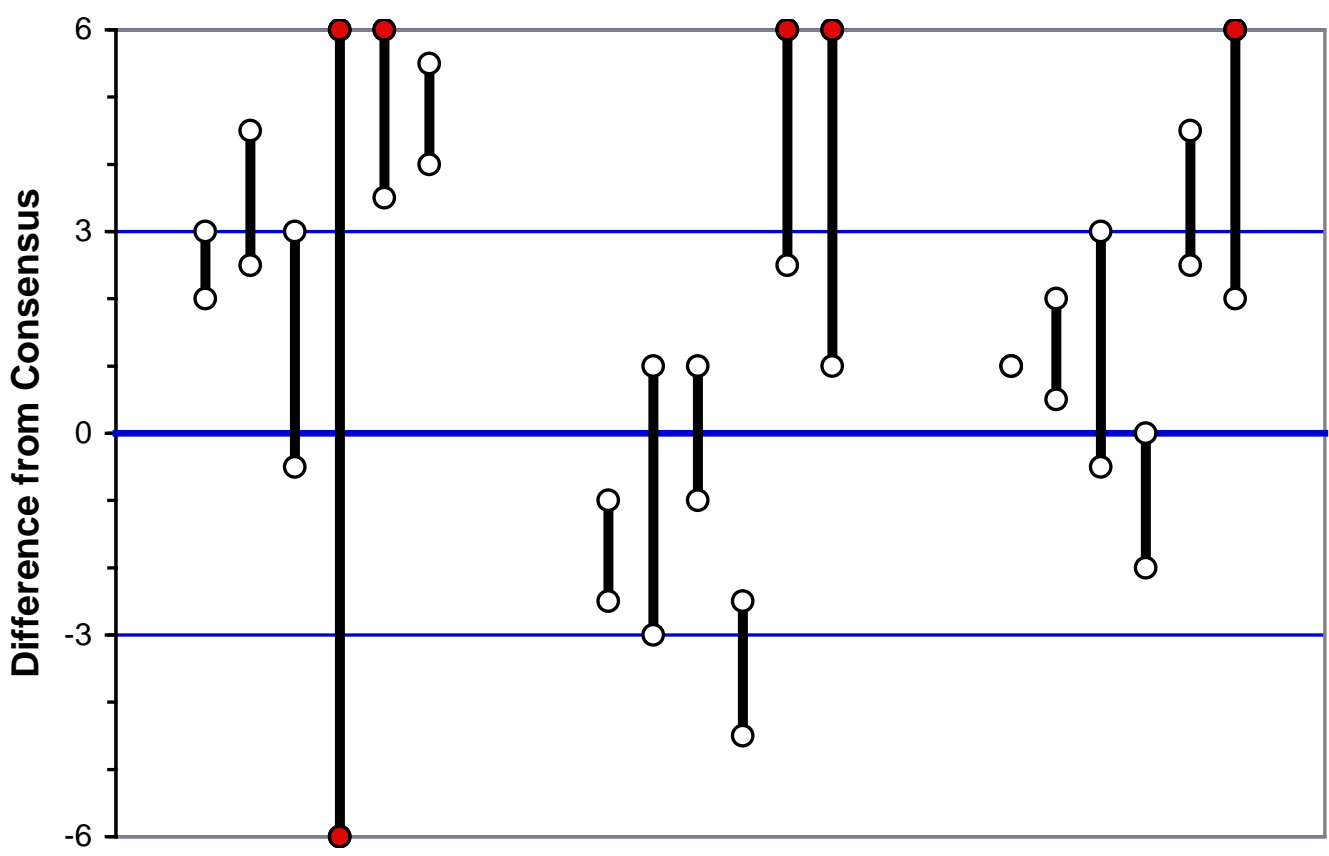
Cereal: A B C D E F
Element:
$\mathrm{Fe}$
A B C D E F
Ca
$\underset{Z n}{A}$ C D F

How to interpret your consensus summary plot:

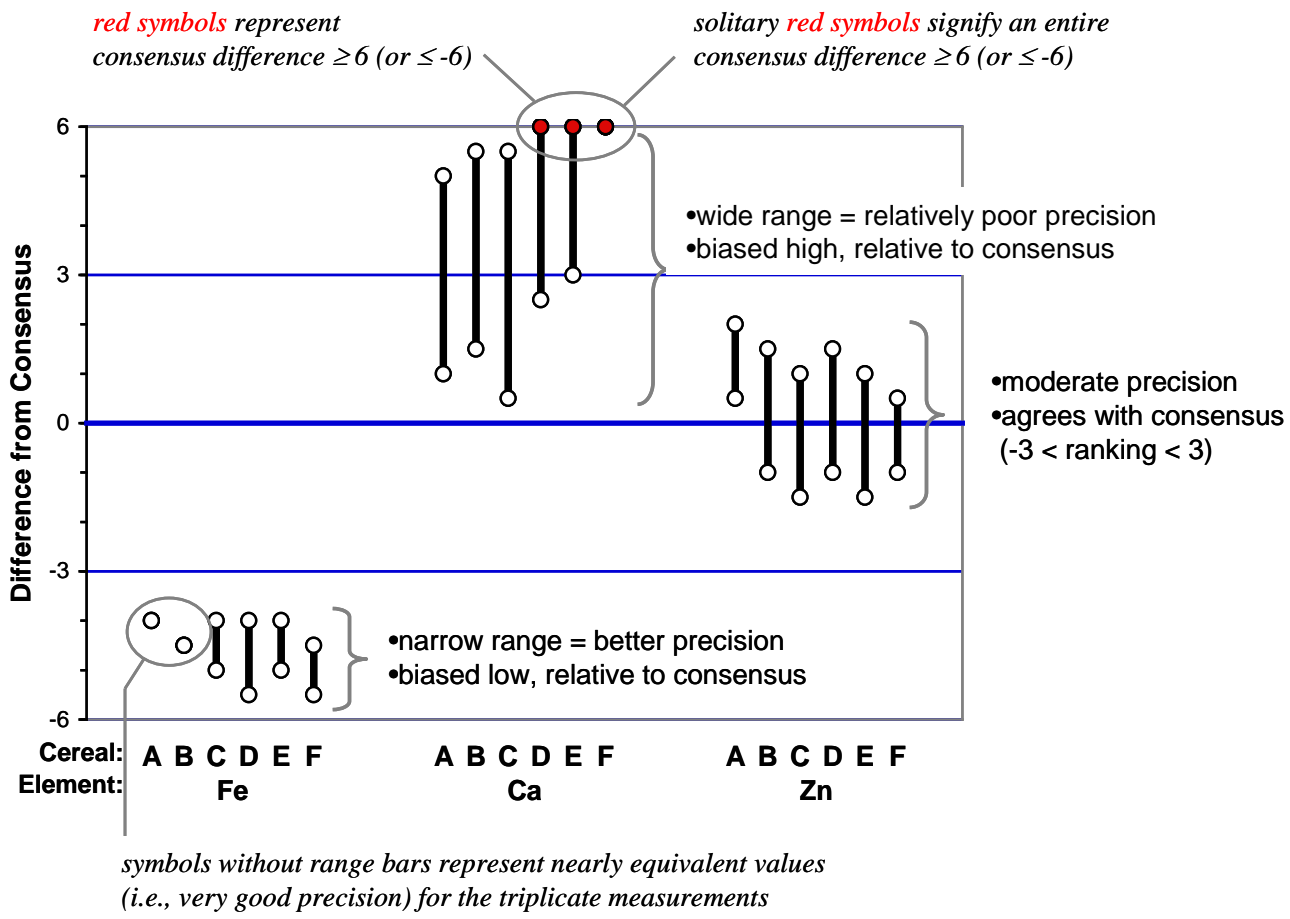




\section{Part II. Your Individual Laboratory Results (Lab Code: E540)}

Your laboratory's data is summarized in Table Z. The mean (average) \pm 1 standard deviation were determined from the reported triplicate $(\mathrm{n}=3$ ) values for each of the 18 measurements ( $\mathrm{Fe}, \mathrm{Ca}$ and $\mathrm{Zn}$ within the six study materials, Cereals A-F) in Exercise E.

Your laboratory's summary consensus plot for $\mathrm{Fe}, \mathrm{Ca}$ and $\mathrm{Zn}$ in the six cereal study materials is provided in Figure Z . The data have been normalized relative to consensus means and Horn-HornDuncan (HHD) uncertainties so that the consensus value is equal to zero. The thick blue line in this plot represents the consensus values for each of the 18 measurements ( $\mathrm{Fe}, \mathrm{Ca}$ and $\mathrm{Zn}$ within the six study materials, Cereals A-F). The thin blue lines represent \pm 3 HHD standard uncertainty intervals about the consensus values. The figure is scaled so that the top and bottom of the $Y$-axis represent \pm 6 HHD intervals. Your laboratory's results for each of the 18 measurements are illustrated by the black bars. The ends of bars that are entirely within the \pm 6 HHD intervals are marked with an open circle $(\circ)$; red circles $(\bullet)$ indicate that some or all of the bar is outside the \pm 6 HHD interval.

The length of each bar indicates how well your measurements agree with themselves. The distance from the center of the bars to the consensus line indicates how well your measurements agree with those of the other participants in this study. (An example consensus plot with explanations is also included to aid in the interpretation your results.) As a general rule, we consider results that fall within \pm 3 HHD standard uncertainty lines to be within consensus (approximate $99 \%$ confidence limit) and results that have a black bar length $\approx 3$ HHD units are considered as acceptable precision. If values are consistently very high or low for a particular element, then a calibration problem may exist. In such cases, it is important to make sure your calibration standards are from a reliable source, the quality is confirmed and that your calibration solutions are correctly prepared and stored. In addition, calibration points must be linear in the expected measurement range and closely surround expected values.

Your laboratory's measurement results for Fe in all materials and $\mathrm{Ca}$ and $\mathrm{Zn}$ in the wheat-based materials (A, B \&C) agree fairly well the consensus means. However, your results for both Ca and $\mathrm{Zn}$ in the rice-based cereal pair ( $\mathrm{D} \& \mathrm{E}$ ) are a factor of two higher than the consensus values. Such significant differences may indicate a sample dilution, calibration, data evaluation, or transcription error. The mean values for all elements in both the ground and flake wheat (B \& C) and rice (D \& E) cereal pairs agree well and the precision of all your measurement processes is quite good (bar lengths of 2 HHD units are less), both indicating that your sample preparation and analysis are quite consistent.

Table Z. Summary of Fe, Ca and Zn values within the six study materials, Cereals A-F

\begin{tabular}{|c|cccccc|}
\hline Lab Code: E540 & \multicolumn{7}{|c|}{ Mean Results $\pm \mathbf{1}$ SD $(\mathbf{n}=\mathbf{3})$} \\
\hline & $\begin{array}{c}\text { Cereal A } \\
\text { Wheat } \\
\text { Ground } \\
(\text { SRM 3233) }\end{array}$ & $\begin{array}{c}\text { Cereal B } \\
\text { Wheat } \\
\text { Ground }\end{array}$ & $\begin{array}{c}\text { Cereal C } \\
\text { Wheat } \\
\text { Flake }\end{array}$ & $\begin{array}{c}\text { Cereal D } \\
\text { Rice } \\
\text { Ground }\end{array}$ & $\begin{array}{c}\text { Cereal E } \\
\text { Rice } \\
\text { Flake }\end{array}$ & $\begin{array}{c}\text { Cereal F } \\
\text { Wheat/Rice } \\
\text { Crushed }\end{array}$ \\
\hline $\mathrm{Fe}(\mu \mathrm{g} / \mathrm{g})$ & $790 \pm 26$ & $847 \pm 15$ & $857 \pm 12$ & $557 \pm 12$ & $543 \pm 6$ & $707 \pm 15$ \\
$\mathrm{Ca}(\mu \mathrm{g} / \mathrm{g})$ & $37130 \pm 380$ & $34230 \pm 250$ & $34800 \pm 350$ & $460 \pm 20$ & $440 \pm 10$ & $18270 \pm 150$ \\
$\mathrm{Zn}(\mu \mathrm{g} / \mathrm{g})$ & $653 \pm 12$ & $707 \pm 6$ & $713 \pm 6$ & $38 \pm 2$ & $41 \pm 5$ & $417 \pm 6$ \\
\hline
\end{tabular}


Figure Z. A consensus plot summarizing your individual results (Lab Code: E540)

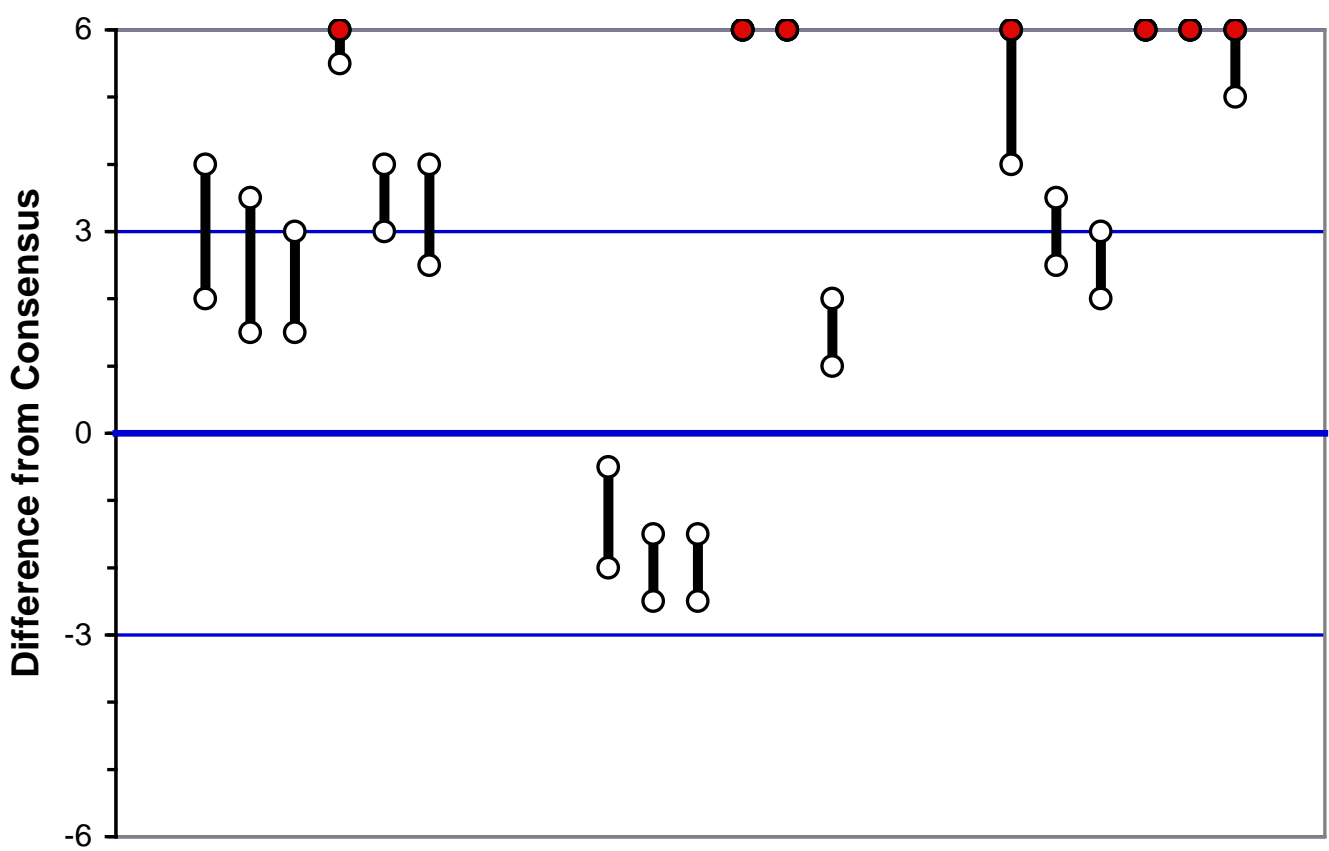
Cereal: A B C D E F
Element:
$\mathrm{Fe}$
A B C D E F
A B C D E F
$\mathrm{Zn}$

How to interpret your consensus summary plot:

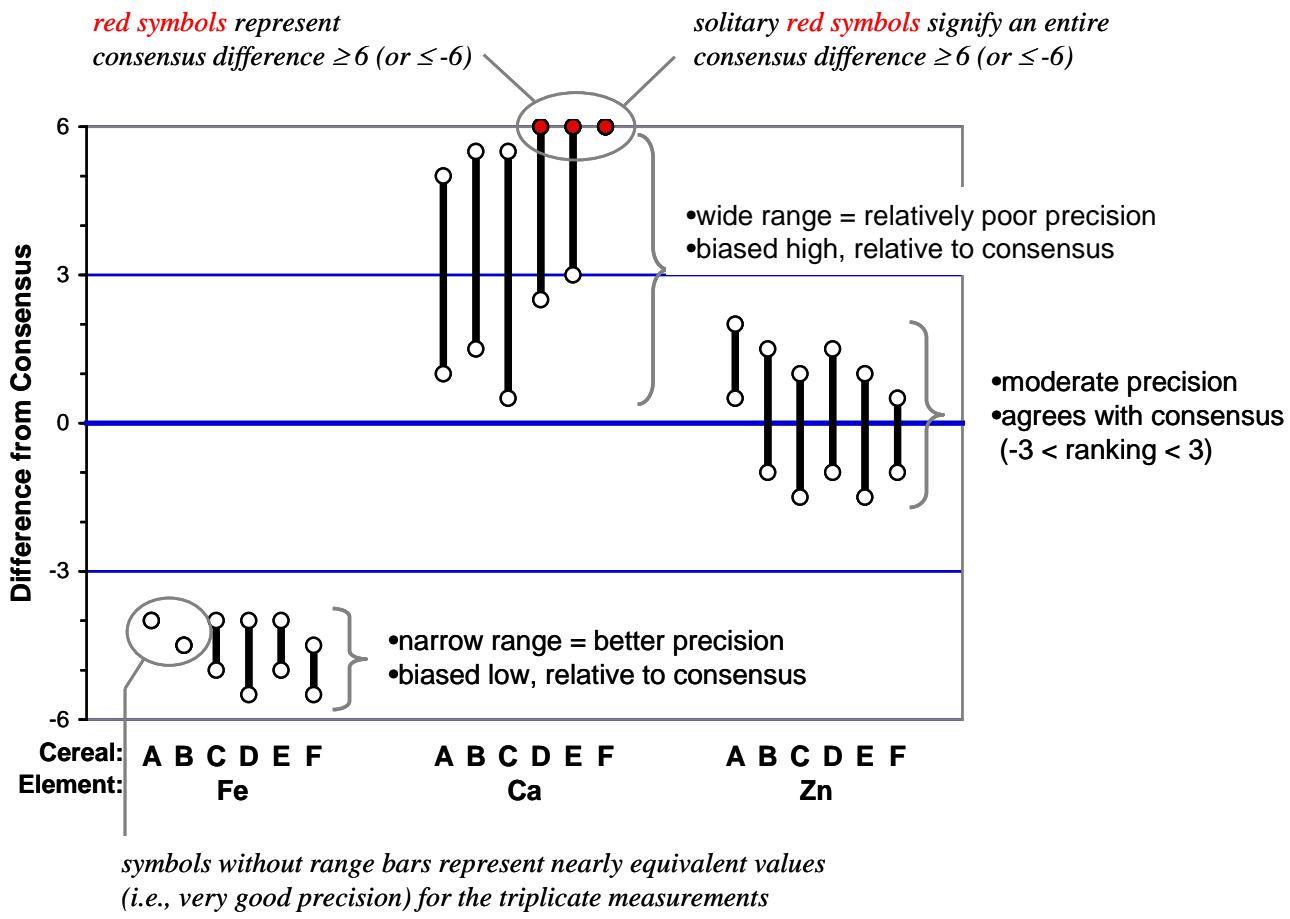




\section{Part II. Your Individual Laboratory Results (Lab Code: E541)}

Your laboratory's data is summarized in Table Z. The mean (average) \pm 1 standard deviation were determined from the reported triplicate $(n=3)$ values for each of the 18 measurements $(\mathrm{Fe}$, $\mathrm{Ca}$ and $\mathrm{Zn}$ within the six study materials, Cereals A-F) in Exercise E.

Your laboratory's summary consensus plot for $\mathrm{Fe}, \mathrm{Ca}$ and $\mathrm{Zn}$ in the six cereal study materials is provided in Figure Z. The data have been normalized relative to consensus means and Horn-HornDuncan (HHD) uncertainties so that the consensus value is equal to zero. The thick blue line in this plot represents the consensus values for each of the 18 measurements (Fe, $\mathrm{Ca}$ and $\mathrm{Zn}$ within the six study materials, Cereals A-F). The thin blue lines represent \pm 3 HHD standard uncertainty intervals about the consensus values. The figure is scaled so that the top and bottom of the $Y$-axis represent \pm 6 HHD intervals. Your laboratory's results for each of the 18 measurements are illustrated by the black bars. The ends of bars that are entirely within the \pm 6 HHD intervals are marked with an open circle $(\circ)$; red circles $(\bullet)$ indicate that some or all of the bar is outside the \pm 6 HHD interval.

The length of each bar indicates how well your measurements agree with themselves. The distance from the center of the bars to the consensus line indicates how well your measurements agree with those of the other participants in this study. (An example consensus plot with explanations is also included to aid in the interpretation your results.) As a general rule, we consider results that fall within \pm 3 HHD standard uncertainty lines to be within consensus (approximate 99\% confidence limit) and results that have a black bar length $\approx 3$ HHD units are considered as acceptable precision. If values are consistently very high or low for a particular element, then a calibration problem may exist. In such cases, it is important to make sure your calibration standards are from a reliable source, the quality is confirmed and that your calibration solutions are correctly prepared and stored. In addition, calibration points must be linear in the expected measurement range and closely surround expected values.

Your laboratory's measurement results for Fe agree well with the consensus means and are quite precise (bars are mostly less than 2 HHD units long). Your Zn results are also precise in all materials, however the values for the relatively high-Zn wheat-based materials (A, B \& C) are somewhat low. Your Ca results show the opposite effect, where your results for the high-Ca content wheat-based materials are somewhat high. These results suggest that you may not be adequately matching of sample mass fractions to the linear range of the calibration curve. The points on your calibration curve extend quite high which would cause possible saturation or a flattening of the linear regression line at the upper points of the curve leading to unreliable results. It is important to prepare samples and calibration points to have appropriate concentration levels and to have enough calibration points to adequately cover the expected concentration range.

Table Z. Summary of Fe, Ca and Zn values within the six study materials, Cereals A-F

\begin{tabular}{|c|cccccc|}
\hline Lab Code: E540 & \multicolumn{5}{|c|}{ Mean Results \pm 1 SD (n= 3) } \\
\hline & $\begin{array}{c}\text { Cereal A } \\
\text { Wheat } \\
\text { Ground } \\
\text { SRM 3233) }\end{array}$ & $\begin{array}{c}\text { Cereal B } \\
\text { Wheat } \\
\text { Ground }\end{array}$ & $\begin{array}{c}\text { Cereal C } \\
\text { Wheat } \\
\text { Flake }\end{array}$ & $\begin{array}{c}\text { Cereal D } \\
\text { Rice } \\
\text { Ground }\end{array}$ & $\begin{array}{c}\text { Cereal E } \\
\text { Rice } \\
\text { Flake }\end{array}$ & $\begin{array}{c}\text { Cereal F } \\
\text { Wheat/Rice } \\
\text { Crushed }\end{array}$ \\
\hline $\mathrm{Fe}(\mu \mathrm{g} / \mathrm{g})$ & $706 \pm 7$ & $779 \pm 8$ & $800 \pm 2$ & $508 \pm 13$ & $501 \pm 12$ & $685 \pm 7$ \\
$\mathrm{Ca}(\mu \mathrm{g} / \mathrm{g})$ & $40240 \pm 940$ & $37810 \pm 300$ & $39920 \pm 530$ & $235 \pm 40$ & $295 \pm 63$ & $25930 \pm 5250$ \\
$\mathrm{Zn}(\mu \mathrm{g} / \mathrm{g})$ & $553 \pm 2$ & $610 \pm 1$ & $624 \pm 2$ & $19 \pm 1$ & $19 \pm 2$ & $358 \pm 5$ \\
\hline
\end{tabular}


Figure Z. A consensus plot summarizing your individual results (Lab Code: E541)

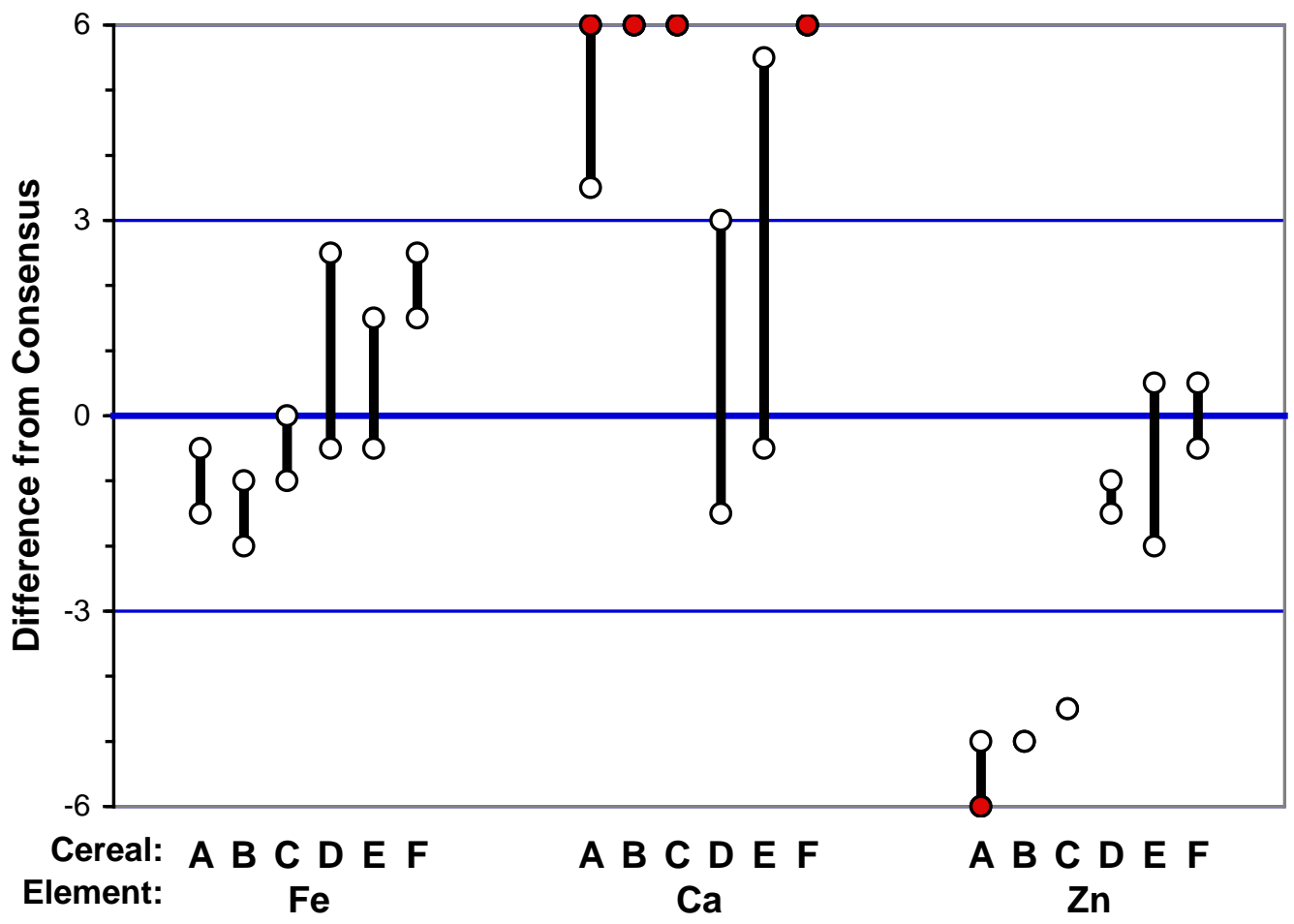

How to interpret your consensus summary plot:

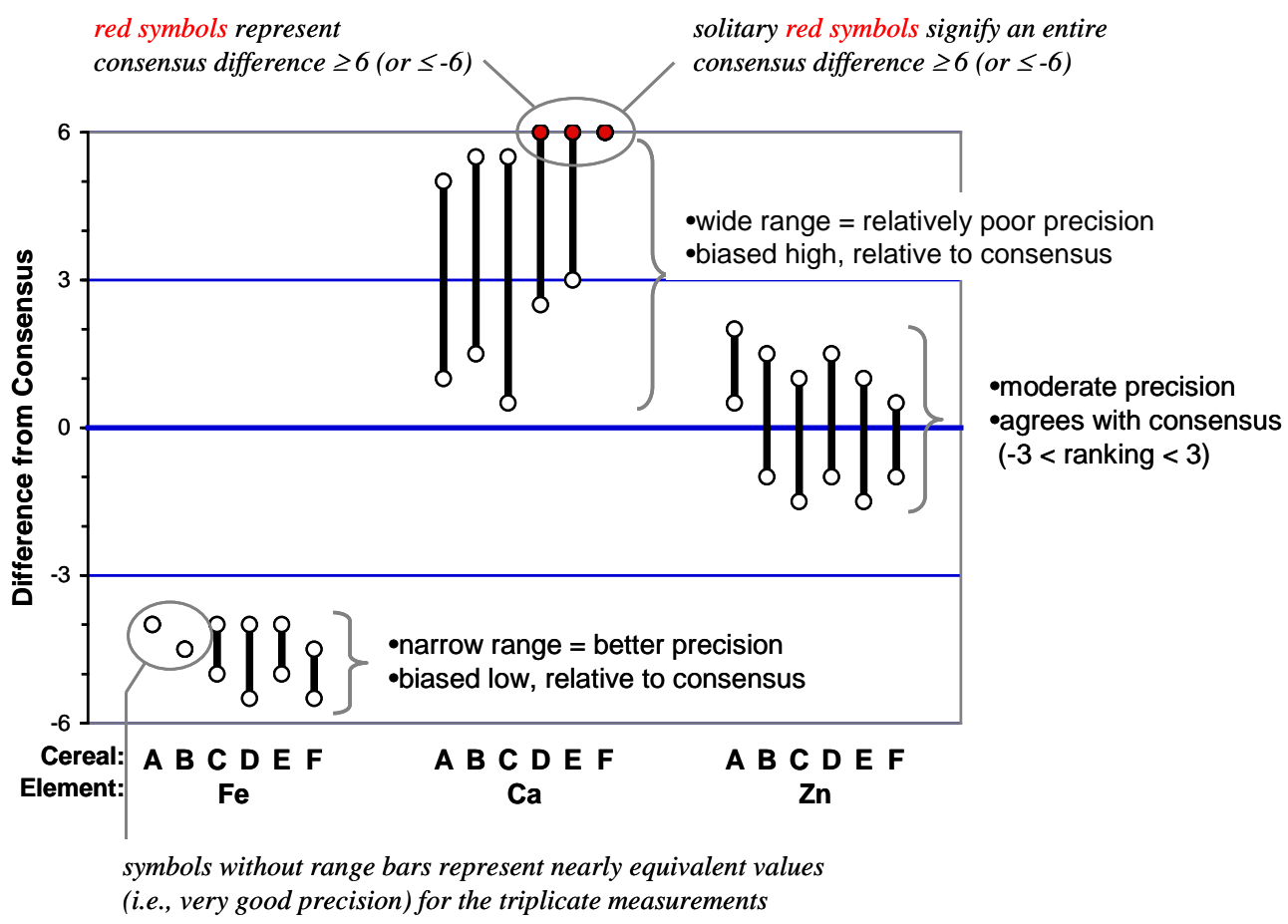

